Polyazamacrocycle ligands facilitate ${ }^{89} \mathrm{Zr}$ radiochemistry and yield ${ }^{89} \mathrm{Zr}$ complexes with remarkable stability.

Darpan N. Pandya*a, Kelly E. Henry ${ }^{b}$, Cynthia S. Day ${ }^{c}$, Stephen A. Graves ${ }^{a}$, Veronica L. Nagle ${ }^{b}$, Thomas R. Dilling $^{b}$, Akesh Sinha ${ }^{a}$, Brandie M. Ehrmann ${ }^{d}$, Nikunj B. Bhatt ${ }^{\mathrm{e}}$, Yusuf Menda ${ }^{\text {, Jason S. Lewis }}{ }^{\mathrm{b} *}$ and Thaddeus J. Wadas ${ }^{\text {a* }}$

a) Department of Radiology University of lowa, lowa City, IA 52242

b) Department of Radiology, Memorial Sloan Kettering Cancer Center, New York, NY 10065

c) Department of Chemistry, Wake Forest University, Winston-Salem, NC 27109.

d) Department of Chemistry, University of North Carolina at Chapel Hill, Chapel Hill, NC 27599.

e) Department of Radiology, Columbia University, New York, NY 10032.

*The corresponding authors for this manuscript are listed below.

Thaddeus J. Wadas, Ph.D.

Associate Professor of Radiology

Director, Small Animal Imaging Core

Carver College of Medicine

University of lowa

169 Newton Road

lowa City, IA 52242

phone: (319) 335-5009

fax: (319) 353-6275

e-mail: thaddeus-wadas@uiowa.edu

Darpan N. Pandya, Ph.D.

Associate Research Scientist

Department of Radiology

Carver College of Medicine

University of lowa

169 Newton Road

lowa City, IA 52242

phone: (319) 335-5161

e-mail: darpan-pandya@uiowa.edu

Jason S. Lewis, Ph.D.

Emily Tow Jackson Chair in Oncology

Department of Radiology,

Memorial Sloan Kettering Cancer Center,

New York, NY 10065

Phone: 646-888-3038

Email: lewisj2@mskcc.org 


\begin{tabular}{|c|c|}
\hline Section & $\begin{array}{l}\text { Page } \\
\text { Number }\end{array}$ \\
\hline Scheme 1. Synthesis of NatZr-TETA & 3 \\
\hline Scheme 2. Synthesis of NatZr-TRITA & 4 \\
\hline HPLC, NMR, HRMS analysis of NatZr-TRITA (Figs. S1-S7) & $5-11$ \\
\hline Scheme 3. Synthesis of NatZr-PCTA & 12 \\
\hline HPLC, NMR, HRMS analysis of NatZr-PCTA (Figs. S8-S14) & 13-19 \\
\hline Scheme 4. Synthesis of NatZr-NOTA-AcAc & 20 \\
\hline HPLC, NMR, HRMS analysis of NatZr-NOTA-AcAc (Figs. S15-S23) & $21-29$ \\
\hline Crystal Structure analysis of NatZr-TRITA (Figs. S24-S30) & $30-38$ \\
\hline 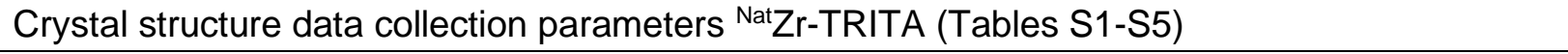 & $39-69$ \\
\hline Crystal Structure analysis of NatZr-PCTA (Figs. S31-S34) & $70-75$ \\
\hline Crystal structure data collection parameters of NatZr-PCTA (Tables S6-S10) & $76-87$ \\
\hline Crystal Structure analysis of NatZr-NOTA (Figs. S35-S41) & $88-96$ \\
\hline Crystal structure data collection parameters NatZr-NOTA (Tables S11-S15) & $97-109$ \\
\hline $\begin{array}{l}\text { Scheme S5. Radiochemical Synthesis of }\left[{ }^{89} \mathrm{Zr}\right] \mathrm{Zr}-\mathrm{PCTA},\left[{ }^{89} \mathrm{Zr}\right] \mathrm{Zr}-\mathrm{NOTA},\left[{ }^{89} \mathrm{Zr}\right] \mathrm{Zr}-\mathrm{TR} \mathrm{RTA} \text { and } \\
{\left[{ }^{89} \mathrm{Zr}\right] \mathrm{Zr}-\mathrm{TETA} \text { using }\left[{ }^{89} \mathrm{Zr}\right] \mathrm{Zr}(\mathrm{ox})_{2}}\end{array}$ & 110 \\
\hline $\begin{array}{l}\text { Summary of optimized radiochemistry conditions to prepare for }\left[{ }^{89} \mathrm{Zr}\right] \mathrm{Zr} \text {-PCTA, }\left[{ }^{89} \mathrm{Zr}\right] \mathrm{Zr} \text {-NOTA, } \\
{\left[{ }^{89} \mathrm{Zr}\right] \mathrm{Zr} \text {-TRITA and }\left[{ }^{89} \mathrm{Zr}\right] \mathrm{Zr}-\mathrm{TETA} \text { using }\left[{ }^{89} \mathrm{Zr}\right] \mathrm{Zr}(\mathrm{ox})_{2}(\mathrm{Table} \mathrm{S} 16)}\end{array}$ & 111 \\
\hline Preparation of $\left[{ }^{89} \mathrm{Zr}\right] \mathrm{ZrCl}{ }_{4}$ from $\left[{ }^{89} \mathrm{Zr}\right] \mathrm{Zr}(\mathrm{ox})_{2}$ (Fig. S42) & 112 \\
\hline $\begin{array}{l}\text { Scheme S6. Radiochemical Synthesis of }\left[{ }^{89} \mathrm{Zr}\right] \mathrm{Zr}-\mathrm{PCTA},\left[{ }^{89} \mathrm{Zr}\right] \mathrm{Zr}-\mathrm{NOTA},\left[{ }^{89} \mathrm{Zr}\right] \mathrm{Zr}-\mathrm{TRITA} \text { and } \\
{\left[{ }^{89} \mathrm{Zr}\right] \mathrm{Zr} \text {-TETA using }\left[{ }^{89} \mathrm{Zr}\right] \mathrm{ZrCl}{ }_{4}}\end{array}$ & 113 \\
\hline Radiochemistry protocol using $\left[{ }^{89} \mathrm{Zr}\right] \mathrm{ZrCl}{ }_{4}$ for $\left[{ }^{89} \mathrm{Zr}\right] \mathrm{Zr}-\mathrm{PCTA}$ (Table S17, Fig. S43) & 114-115 \\
\hline Quality control of [ $\left.{ }^{89} \mathrm{Zr}\right] \mathrm{Zr}$-PCTA by radio-TLC (Fig. S44) & 116 \\
\hline Quality control of [ $\left.{ }^{89} \mathrm{Zr}\right] \mathrm{Zr}-\mathrm{PCTA}$ by radio-HPLC (Fig. S45) & 117 \\
\hline Radiochemistry protocol using $\left[{ }^{89} \mathrm{Zr}\right] \mathrm{ZrCl} 4$ for $\left[{ }^{89} \mathrm{Zr}\right] \mathrm{Zr}$-NOTA (Table S18, Fig. S46) & 118-119 \\
\hline Quality control of [ $\left.{ }^{89} \mathrm{Zr}\right] \mathrm{Zr}$-NOTA by radio-TLC (Fig. S47) & 120 \\
\hline Quality control of [ $\left.{ }^{89} \mathrm{Zr}\right] \mathrm{Zr}$-NOTA by radio-HPLC (Fig. S48) & 121 \\
\hline Radiochemistry protocol using $\left[{ }^{89} \mathrm{Zr}\right] \mathrm{ZrCl}_{4}$ for $\left[{ }^{89} \mathrm{Zr}\right] \mathrm{Zr}$-TRITA (Tables S19, S20; Figs. S49, S50) & $122-125$ \\
\hline Quality control of [ $\left.{ }^{89} \mathrm{Zr}\right] \mathrm{Zr}$-TRITA by radio-TLC (Fig. S51) & 126 \\
\hline Quality control of $\left[{ }^{89} \mathrm{Zr}\right] \mathrm{Zr}$-TRITA by radio-HPLC (Fig. S52) & 127 \\
\hline Radiochemistry protocol using $\left[{ }^{89} \mathrm{Zr}\right] \mathrm{ZrCl}_{4}$ for $\left[{ }^{89} \mathrm{Zr}\right] \mathrm{Zr}$-TETA (Table S21, Fig. S53) & $128-129$ \\
\hline Determination of partition coefficients (LogP) (Table S22) & 130 \\
\hline In vitro serum stability study by SE-HPLC (Figs. S54, S55) & 131-132 \\
\hline $\begin{array}{l}\left.\text { Complete Biodistribution studies of }{ }^{89} \mathrm{Zr}\right] \mathrm{Zr} \text {-PCTA (Table S23), }\left[{ }^{89} \mathrm{Zr}\right] \mathrm{Zr} \text {-NOTA (Table S24), and } \\
{\left[{ }^{89} \mathrm{Zr}\right] \mathrm{Zr} \text {-TRITA (Table S25) }}\end{array}$ & $133-135$ \\
\hline Summary of comparative biodistribution data of [ $\left.{ }^{89} \mathrm{Zr}\right] \mathrm{Zr}$-complexes (Figs. S56-S58) & $136-138$ \\
\hline Dynamic PET data of [ $\left.{ }^{89} \mathrm{Zr}\right] \mathrm{Zr}-\mathrm{PCTA}$ and $\left[{ }^{89} \mathrm{Zr}\right] \mathrm{Zr}-\mathrm{NOTA}$ in specific tissues (Fig. S59) & 139 \\
\hline $\begin{array}{l}\text { Tables comparing in vitro and in vivo properties of }\left[{ }^{89} \mathrm{Zr}\right] \mathrm{Zr} \text {-PCTA, }\left[{ }^{89} \mathrm{Zr}\right] \mathrm{Zr}-\mathrm{NOTA} \text {, and }\left[{ }^{89} \mathrm{Zr}\right] \mathrm{Zr}- \\
\text { TRITA with }\left[{ }^{89} \mathrm{Zr}\right] \mathrm{Zr} \text {-Complexes reported in the literature (Tables S26-S28) }\end{array}$ & $140-142$ \\
\hline References & 143-144 \\
\hline
\end{tabular}




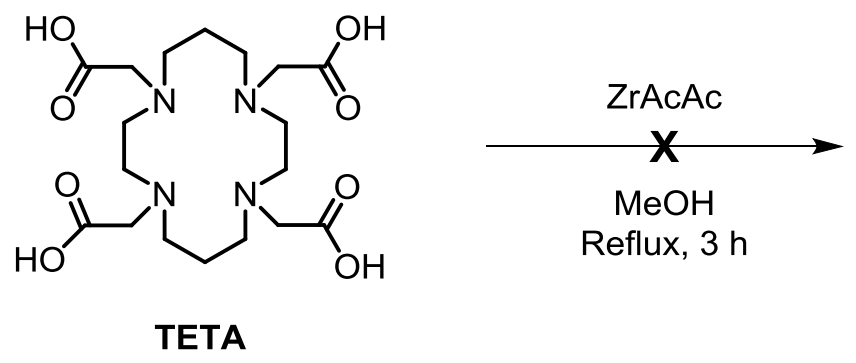

Scheme S1. Synthesis of zirconium complex of TETA 

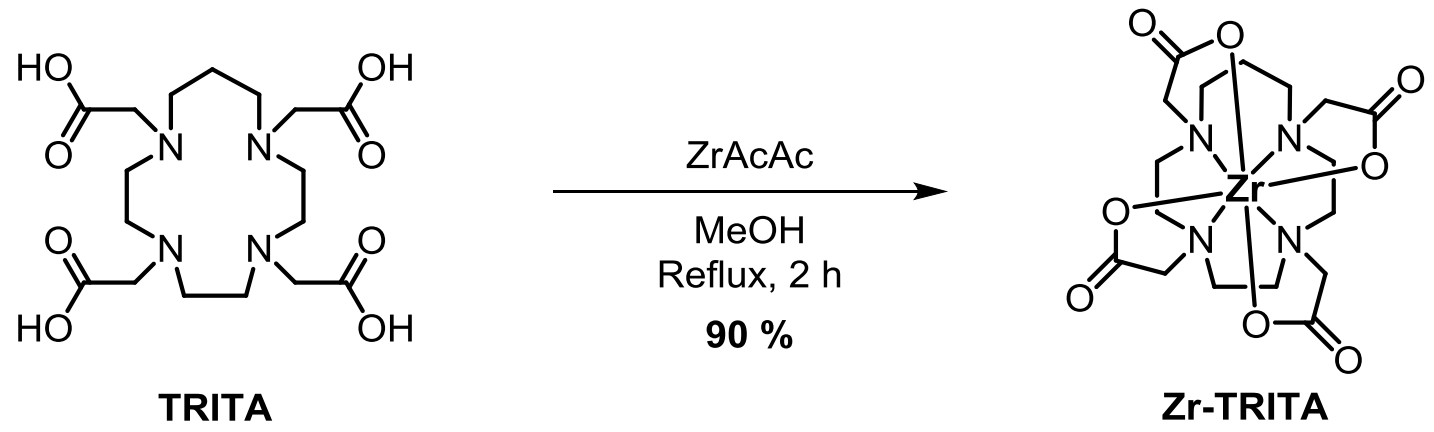

Scheme S2. Synthesis of zirconium complex of TRITA 


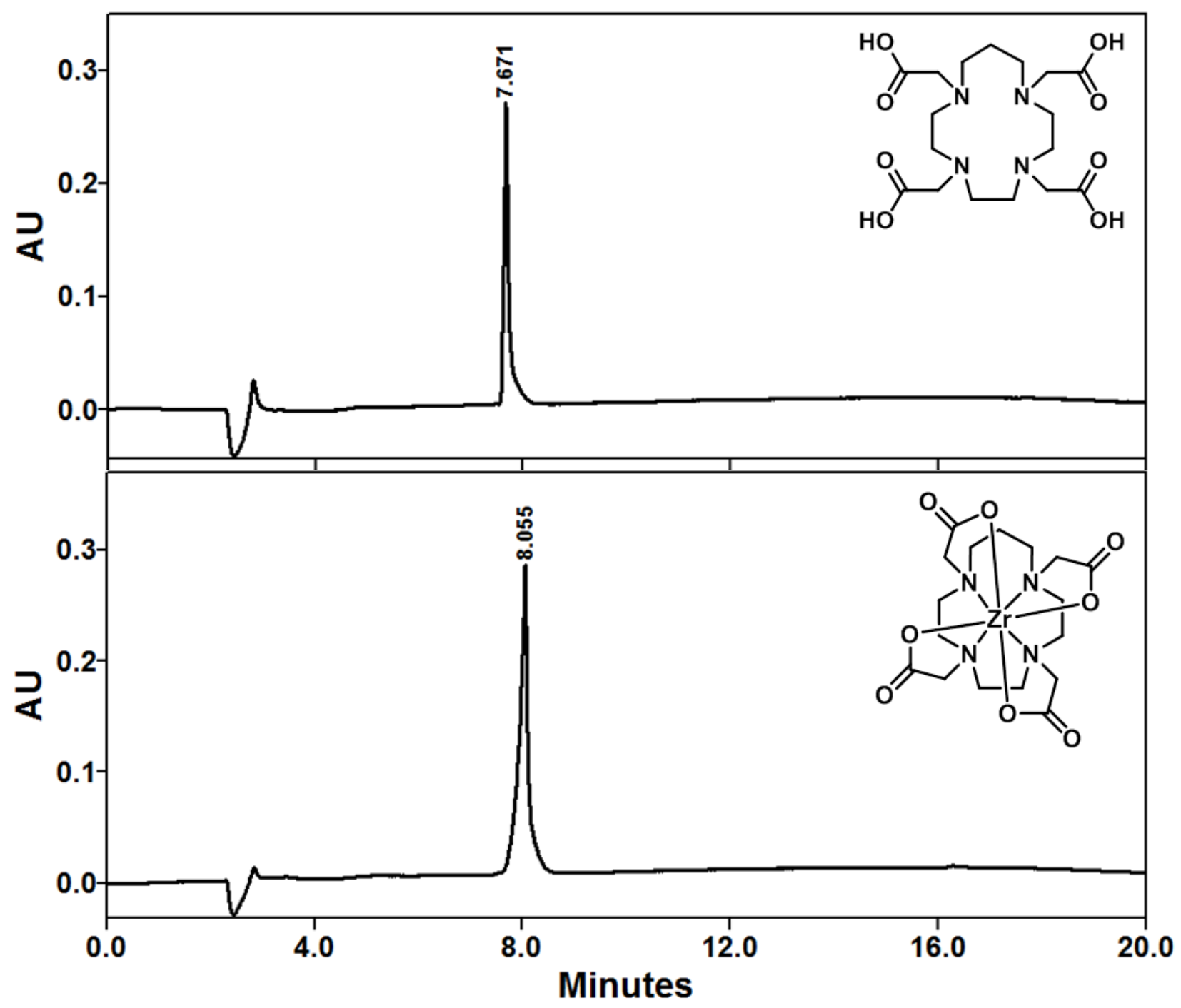

Figure S1. UV-HPLC chromatogram $(201 \mathrm{~nm})$ of TRITA ligand (top) and nonradioactive NatZr-TRITA complex (bottom) 


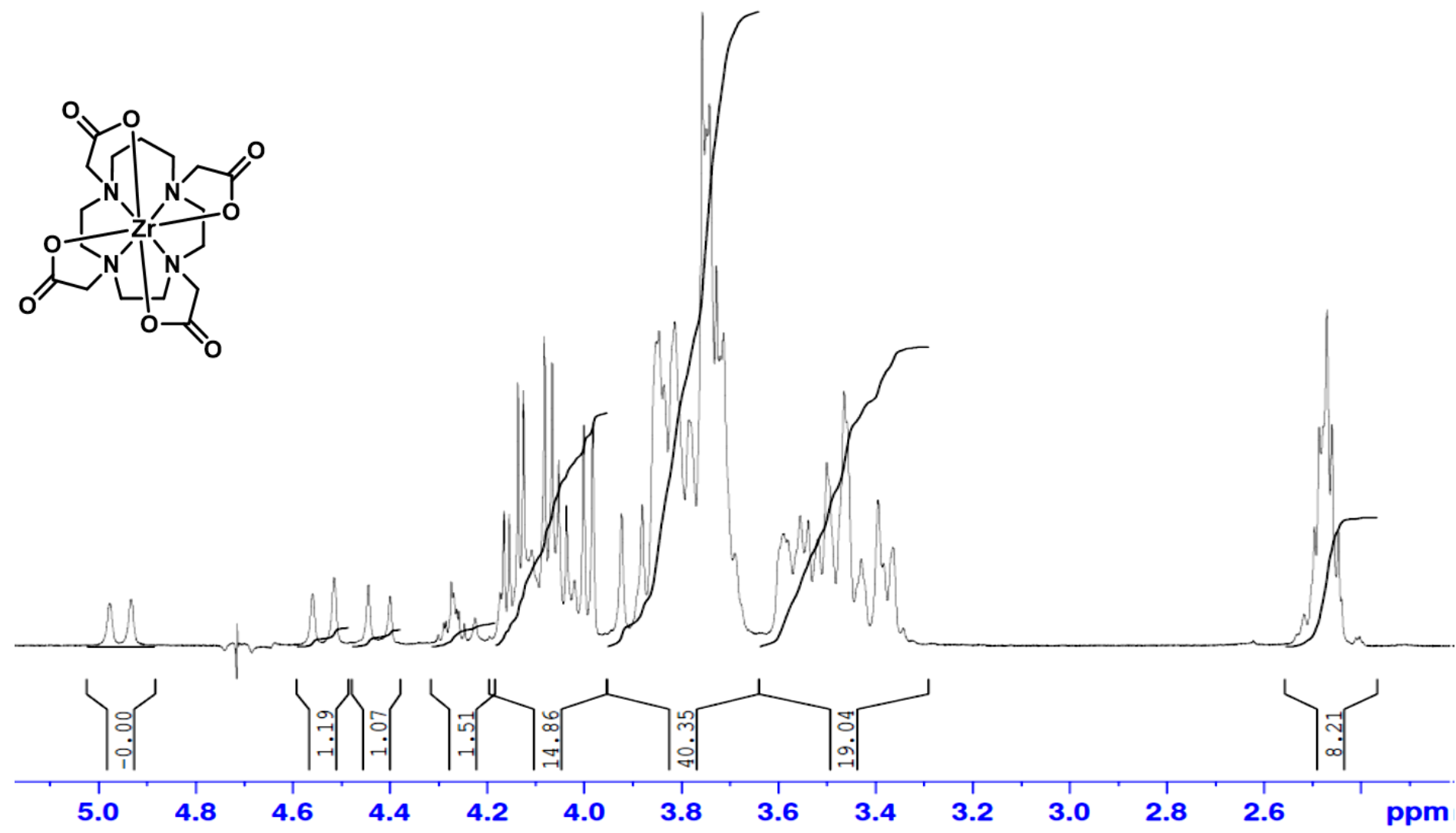

Figure S2. ${ }^{1} \mathrm{H}-\mathrm{NMR}$ spectrum $\left(\mathrm{D}_{2} \mathrm{O}-400 \mathrm{MHz}\right)$ of the $\mathrm{Zr}$-TRITA complex 

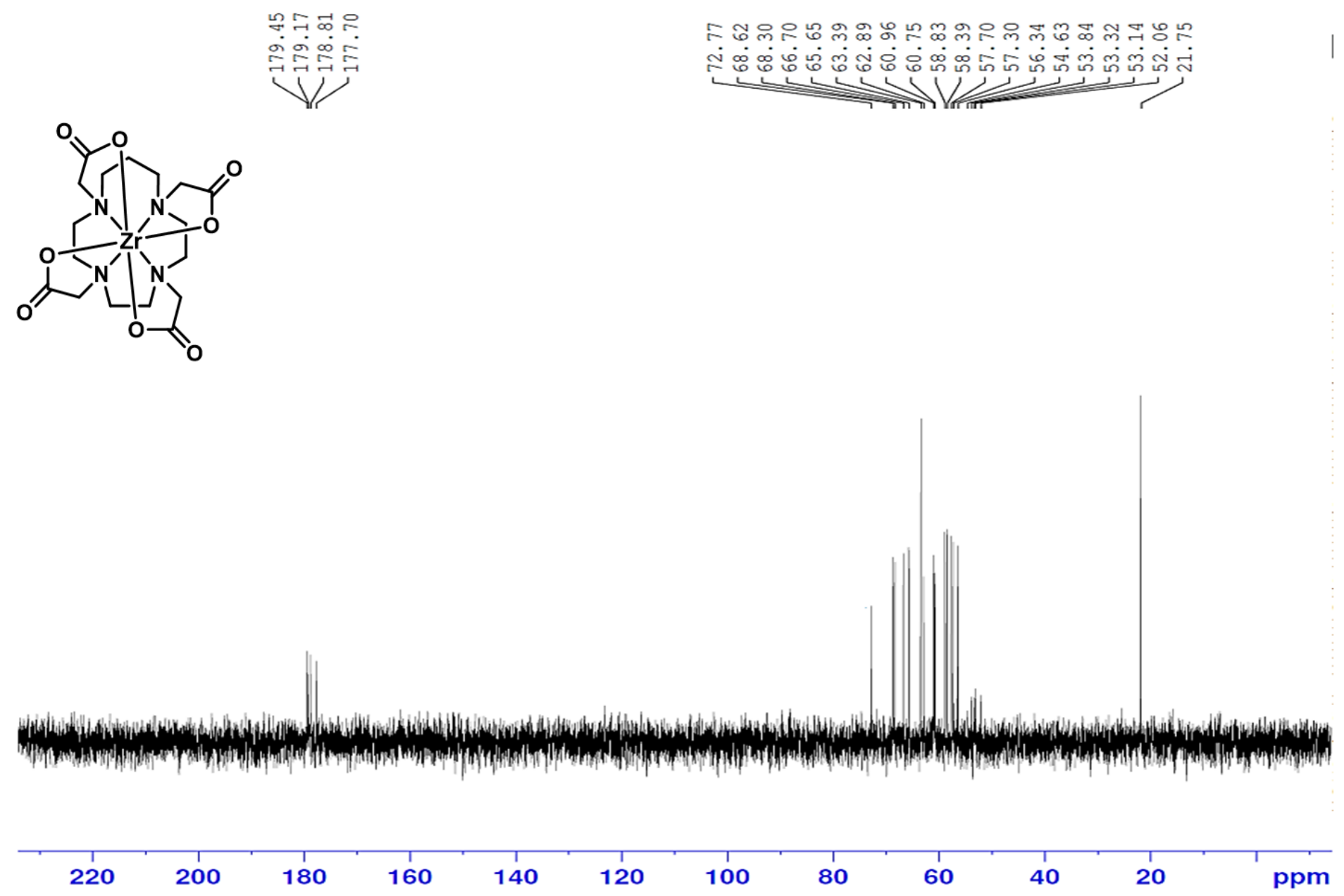

Figure S3. ${ }^{13} \mathrm{C}-\mathrm{NMR}$ spectrum $\left(\mathrm{D}_{2} \mathrm{O}-101 \mathrm{MHz}\right)$ of the $\mathrm{Zr}$-TRITA complex 


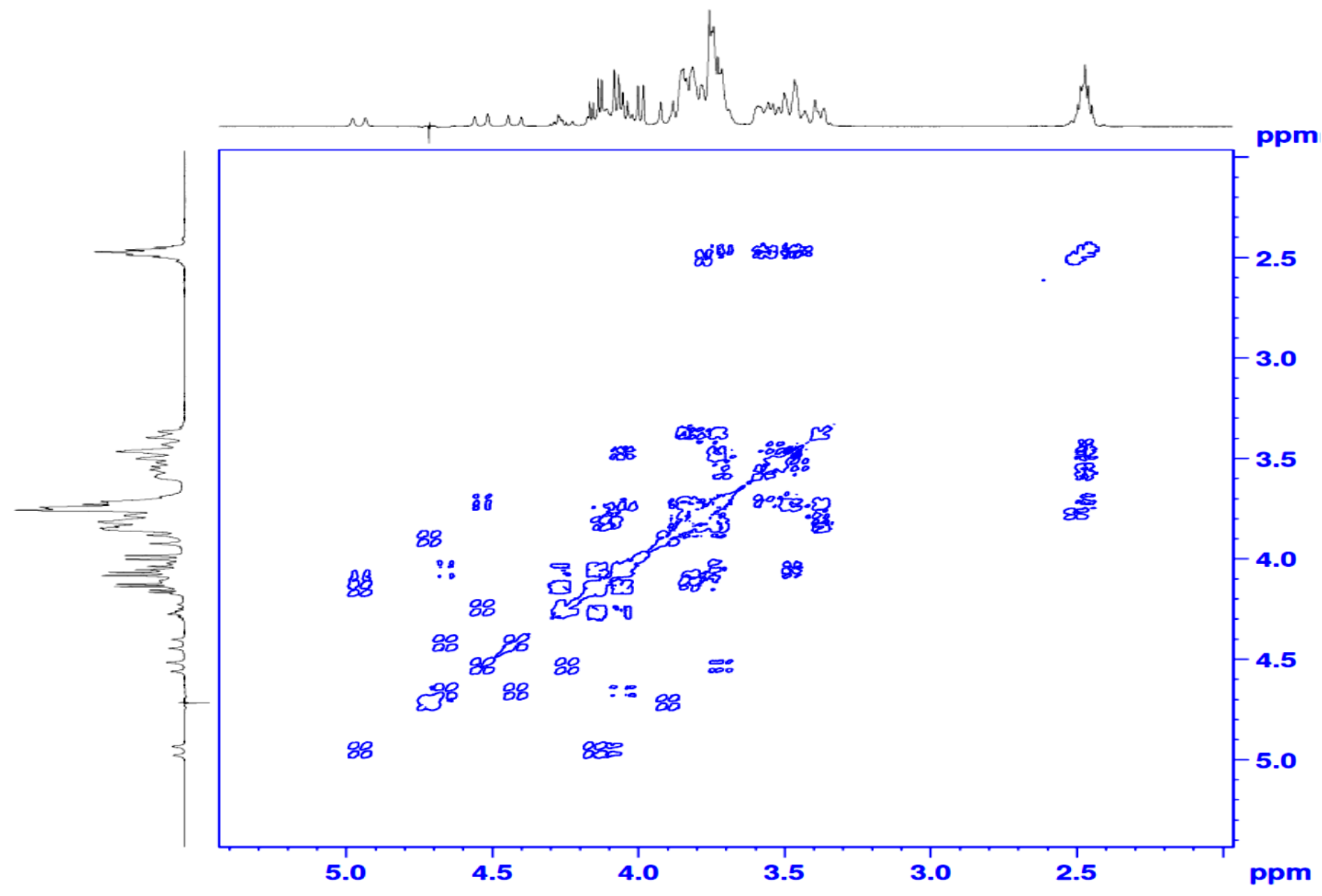

Figure S4. 2-D COSY NMR spectrum $\left(\mathrm{D}_{2} \mathrm{O}-400 \mathrm{MHz}\right)$ of the Zr-TRITA complex 


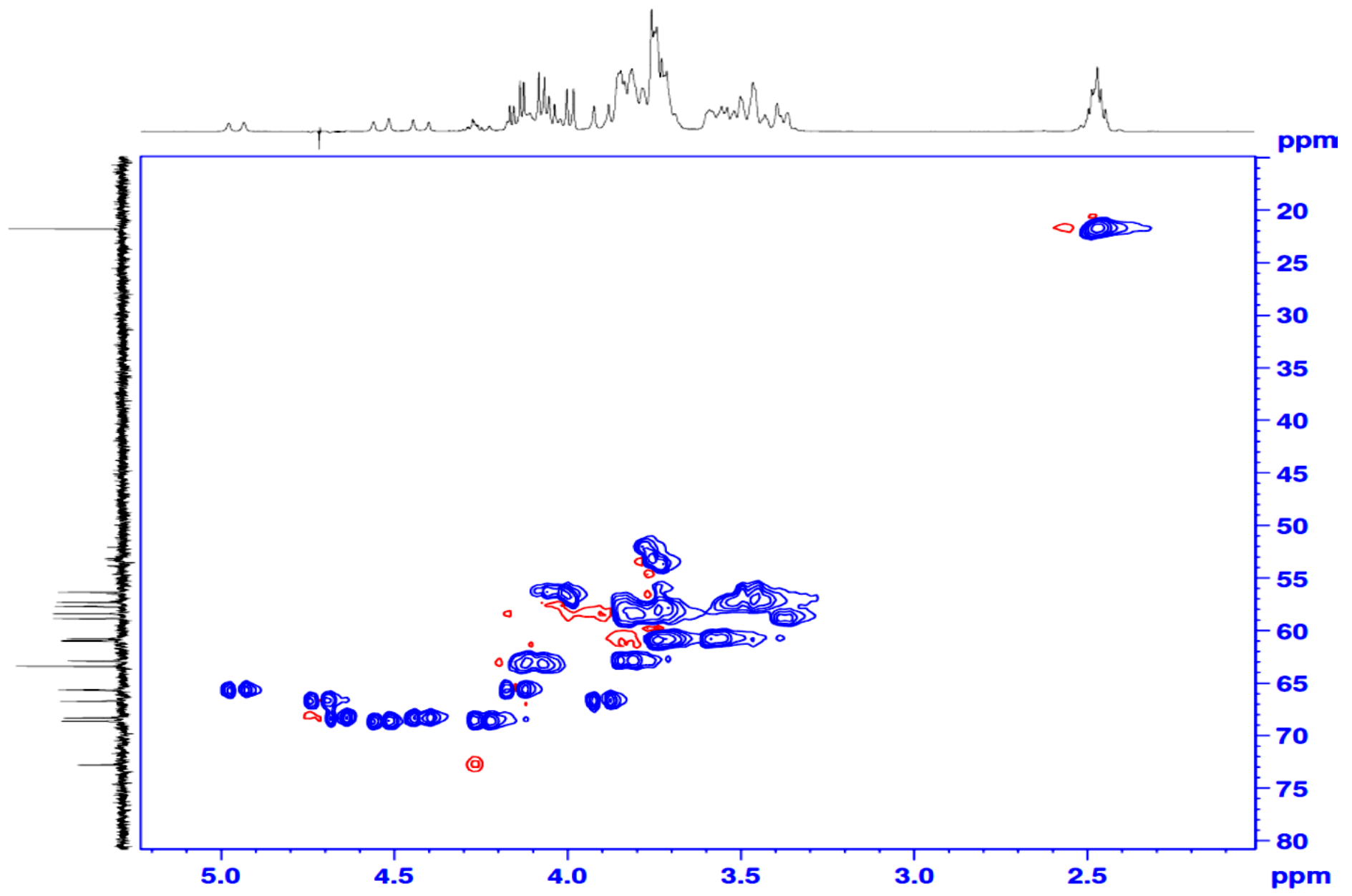

Figure S5. 2-D HSQC NMR spectrum $\left(\mathrm{D}_{2} \mathrm{O}-400 \mathrm{MHz}\right)$ of the Zr-TRITA complex 


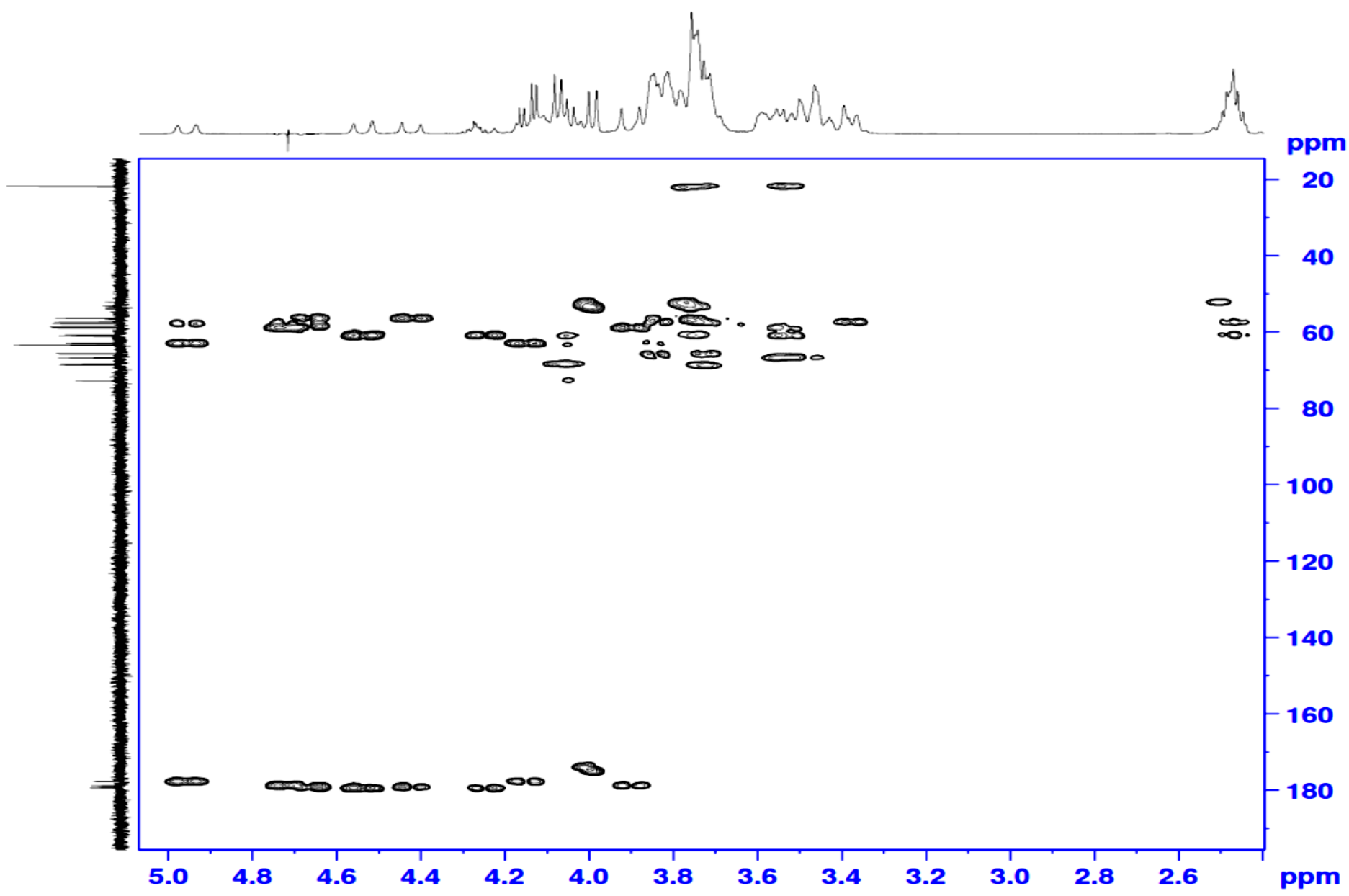

Figure S6. 2-D HMBC NMR spectrum $\left(\mathrm{D}_{2} \mathrm{O}-400 \mathrm{MHz}\right)$ of the Zr-TRITA complex 


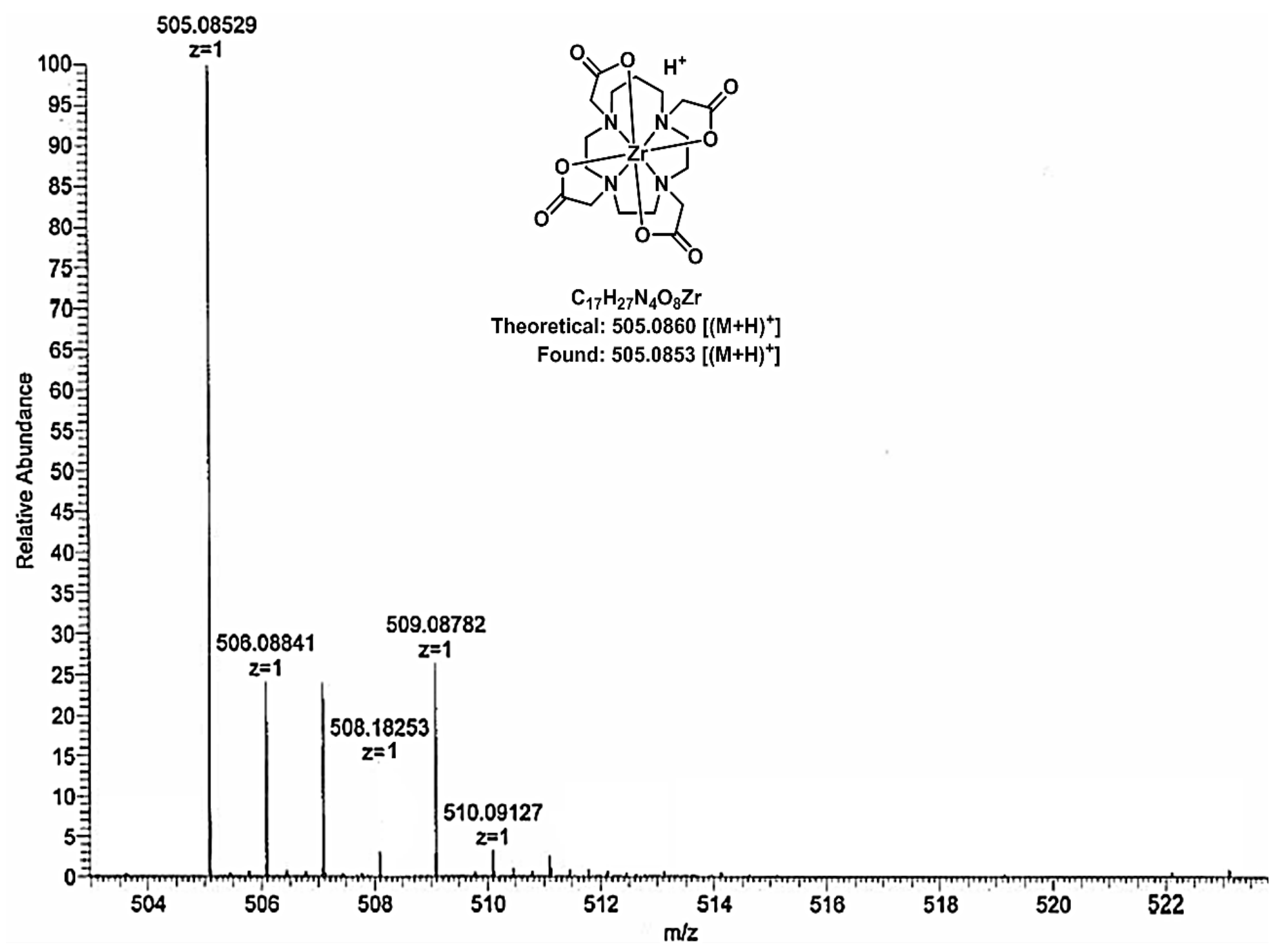

Figure S7. HR ESI FT-ICR MS (Positive mode) analysis of the Zr-TRITA complex 

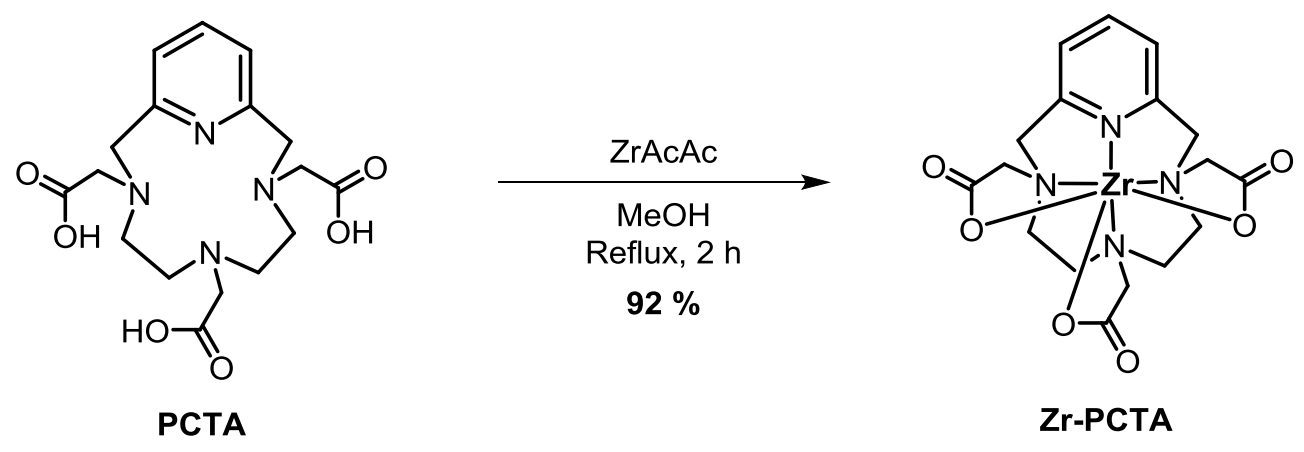

Scheme S3. Synthesis of zirconium complex of PCTA 


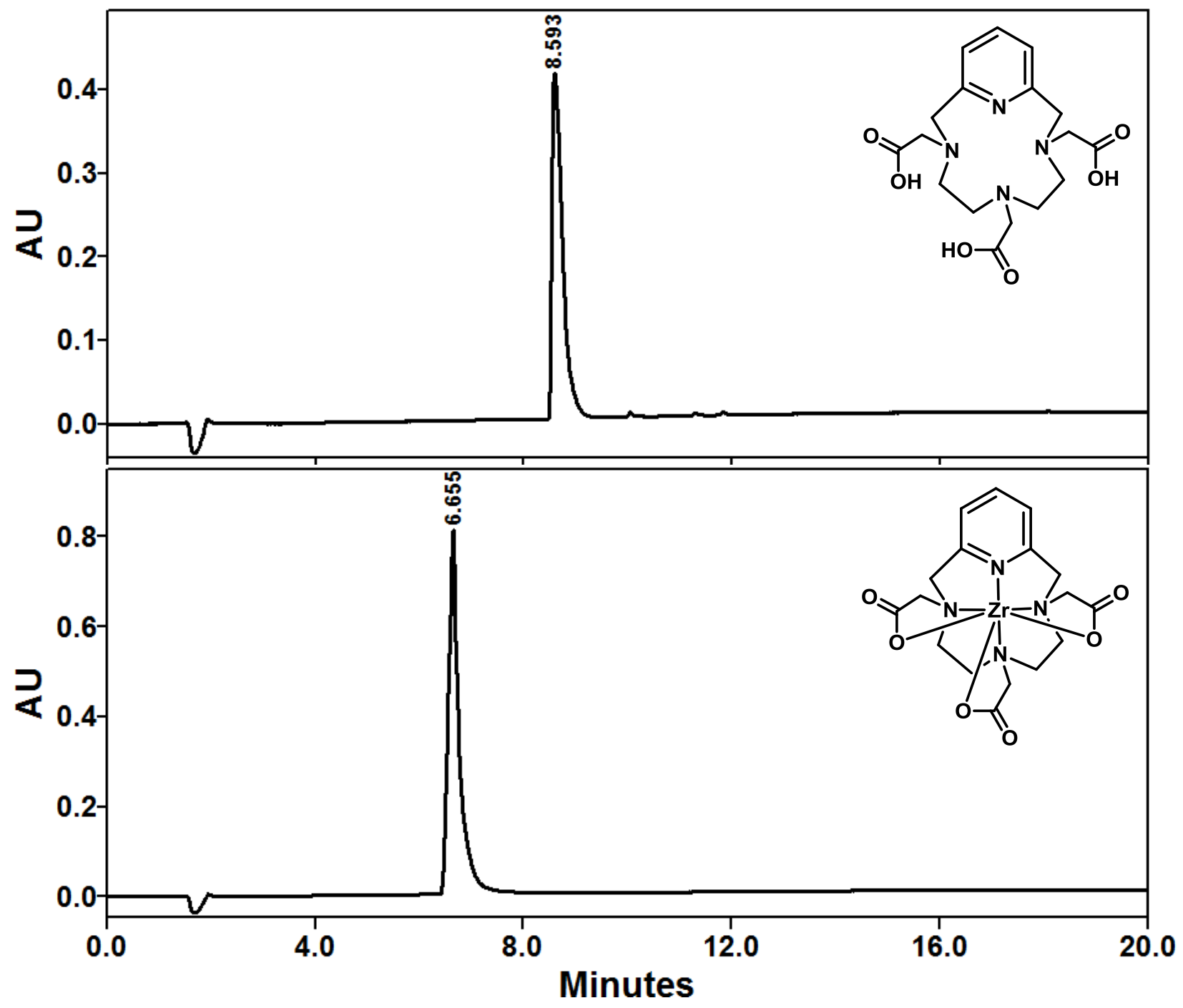

Figure S8. UV-HPLC chromatogram (201nm) of PCTA ligand (top) and nonradioactive NatZr-PCTA complex (bottom) 


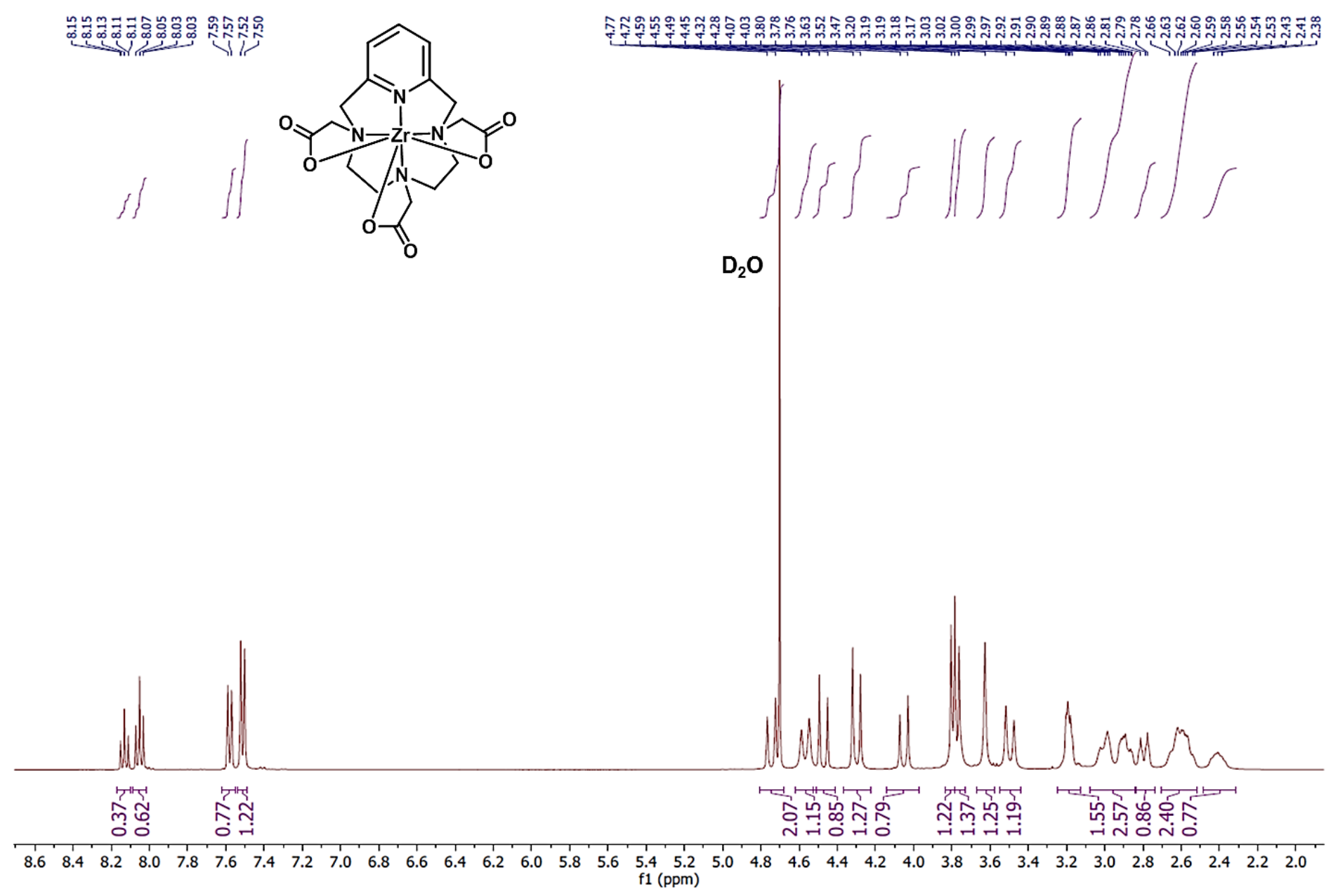

Figure S9. ${ }^{1} \mathrm{H}-\mathrm{NMR}$ spectrum $\left(\mathrm{D}_{2} \mathrm{O}-400 \mathrm{MHz}\right)$ of the $\mathrm{Zr}$-PCTA complex 


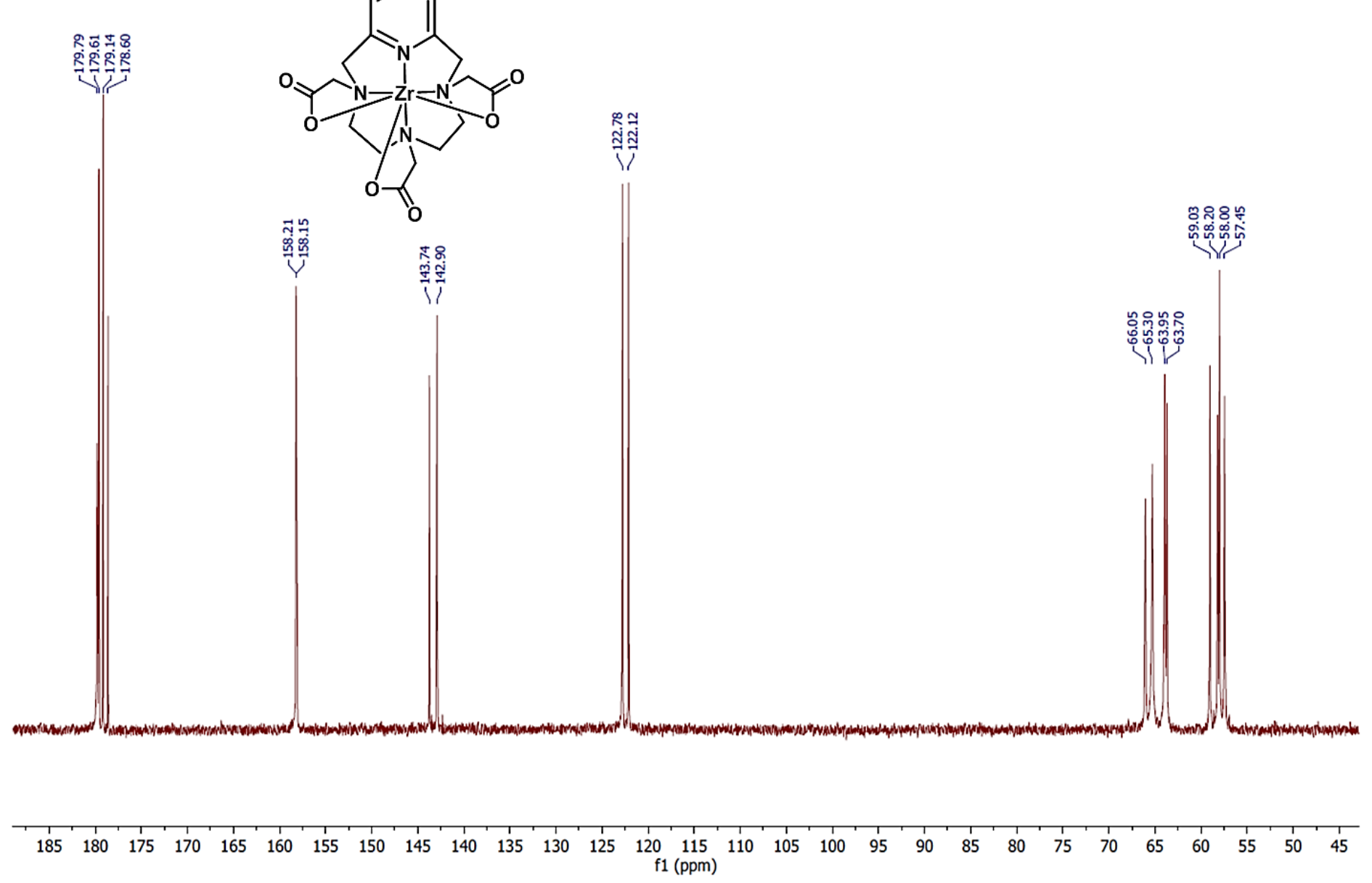

Figure $\mathrm{S} 10 .{ }^{13} \mathrm{C}$-NMR spectrum $\left(\mathrm{D}_{2} \mathrm{O}-101 \mathrm{MHz}\right)$ of the Zr-PCTA complex 


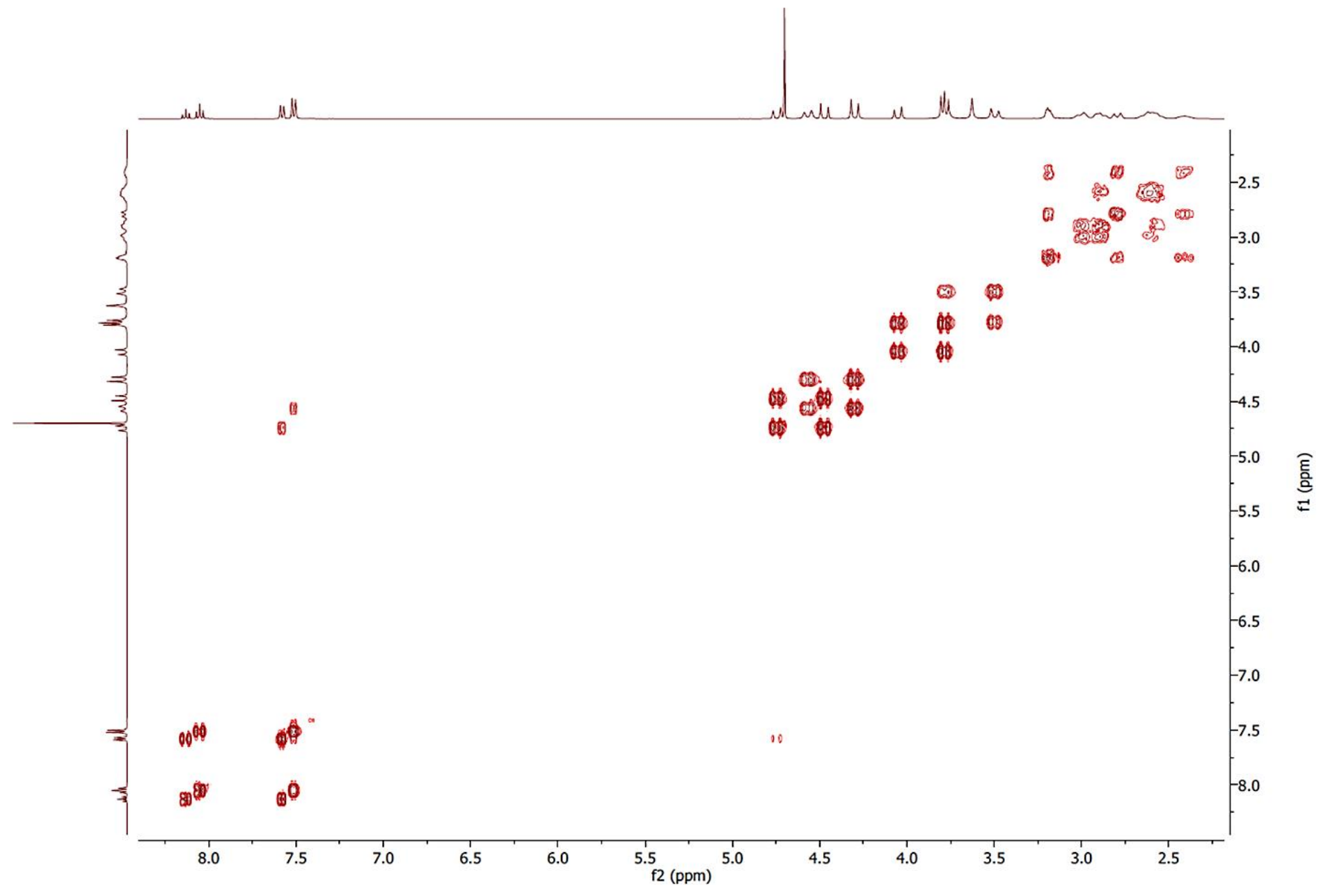

Figure S11. 2-D COSY NMR spectrum $\left(\mathrm{D}_{2} \mathrm{O}-400 \mathrm{MHz}\right)$ of the Zr-PCTA complex 


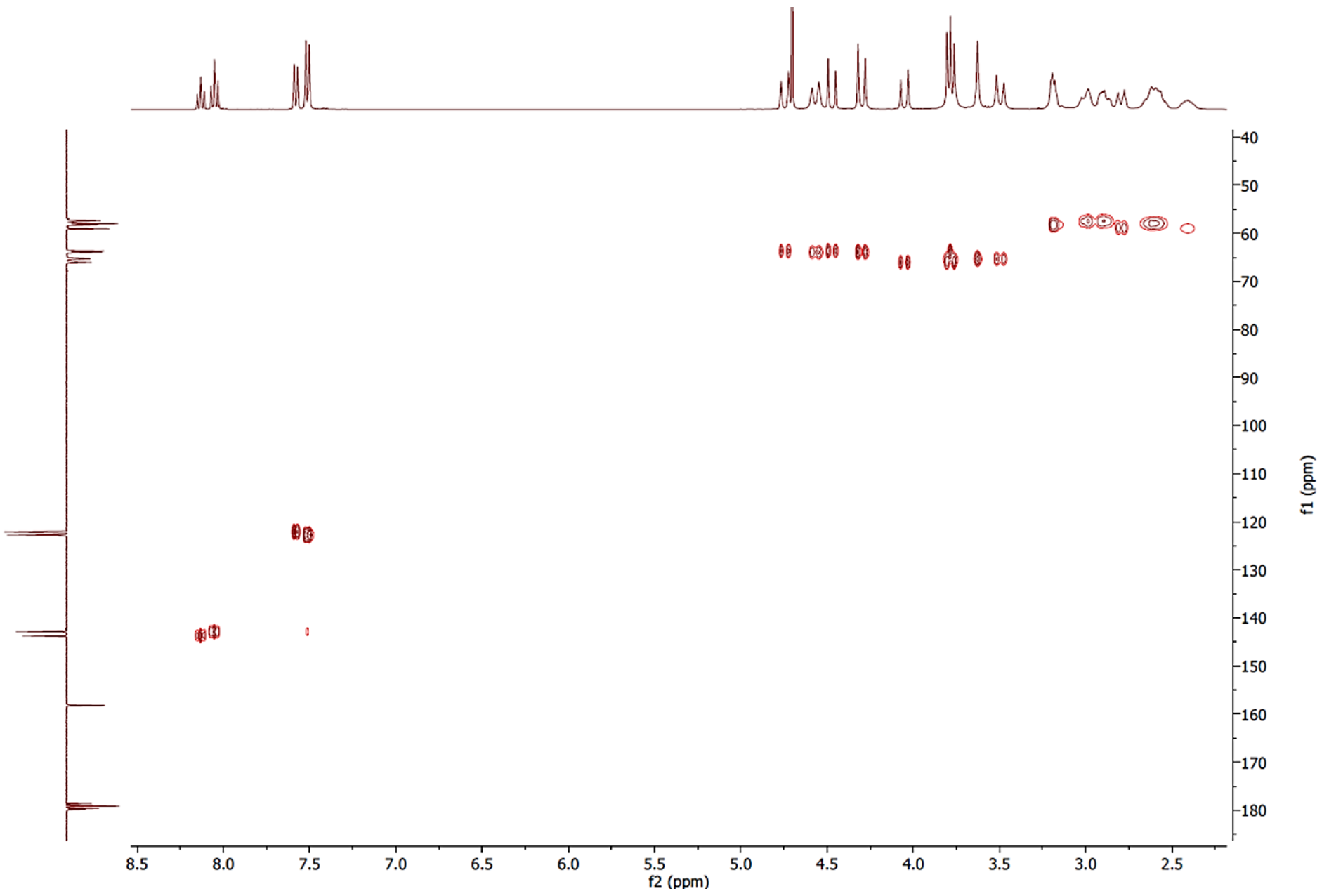

Figure S12. 2-D HSQC NMR spectrum $\left(\mathrm{D}_{2} \mathrm{O}-400 \mathrm{MHz}\right)$ of the Zr-PCTA complex 


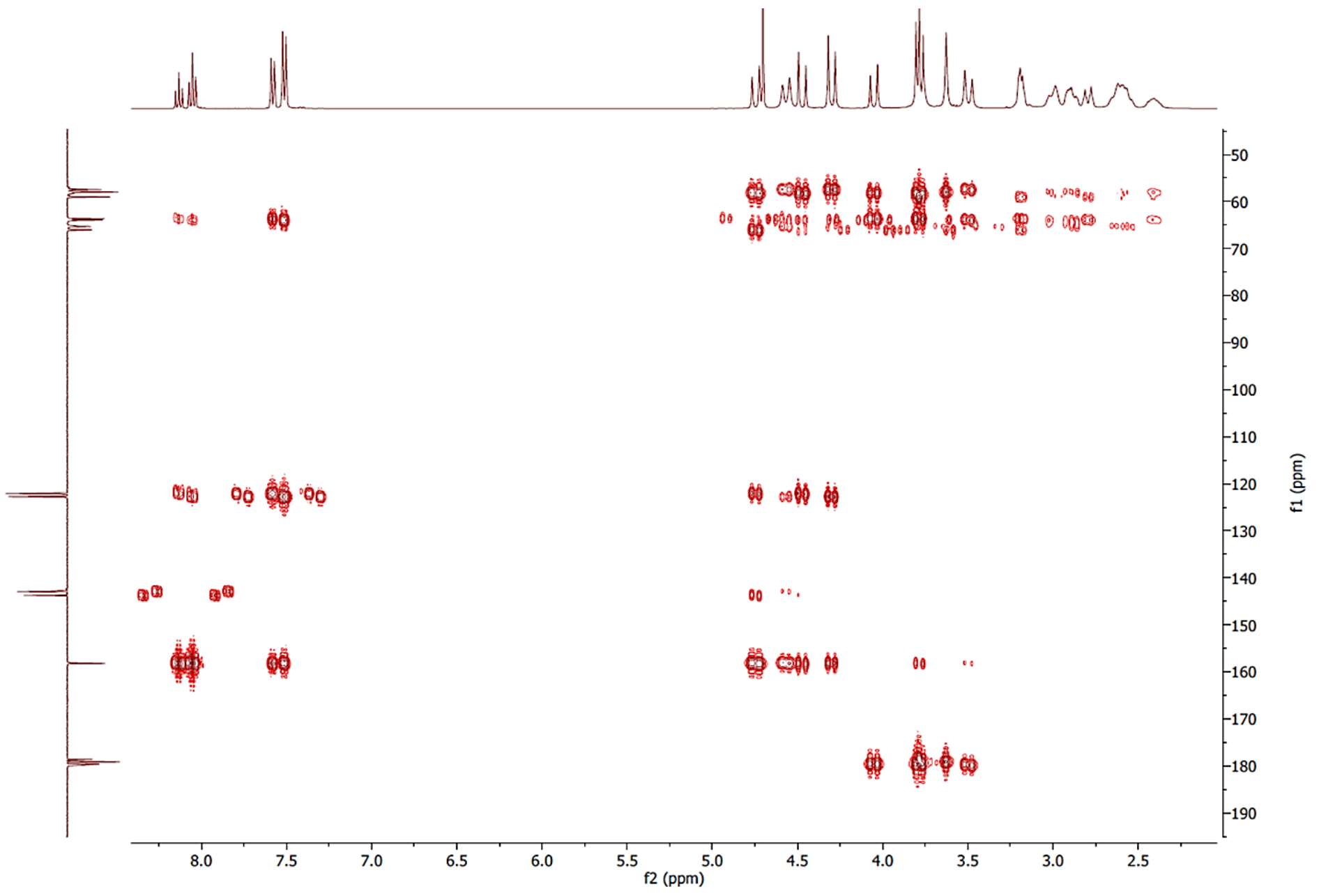

Figure S13. 2-D HMBC NMR spectrum $\left(\mathrm{D}_{2} \mathrm{O}-400 \mathrm{MHz}\right)$ of the Zr-PCTA complex 


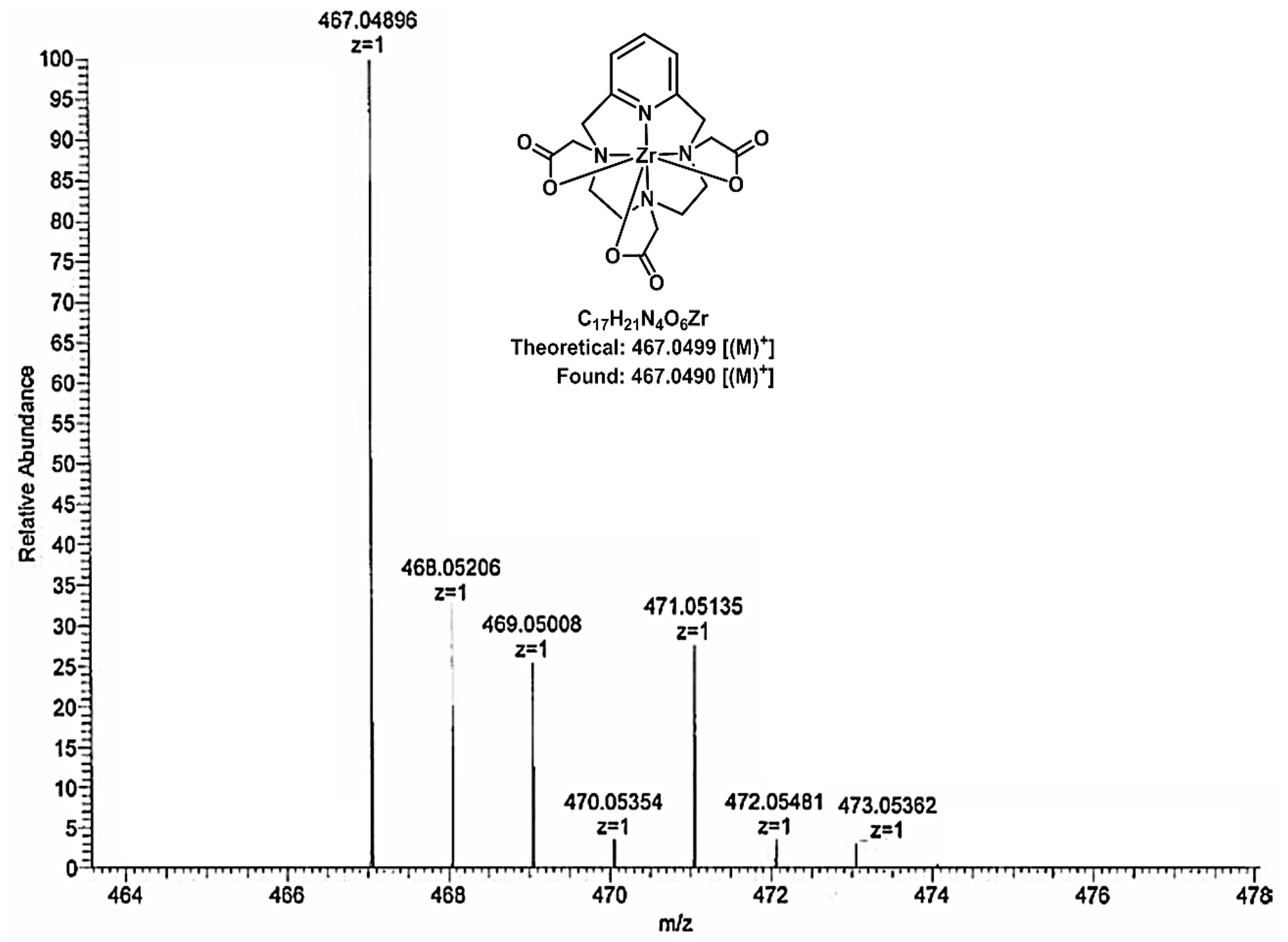

Figure S14. HR ESI FT-ICR MS (Positive mode) analysis of the Zr-PCTA complex 


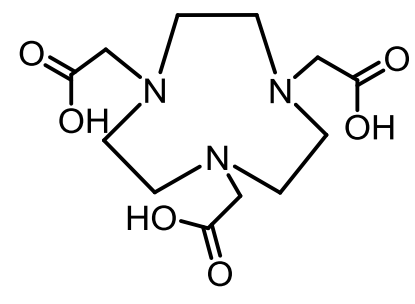

NOTA

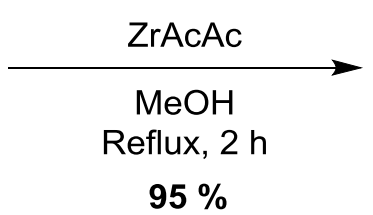

$95 \%$

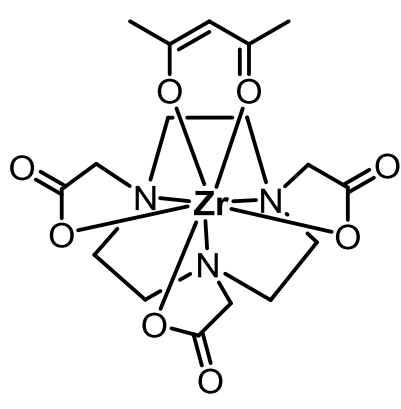

Zr-NOTA

Scheme S4. Synthesis of zirconium complex of NOTA 


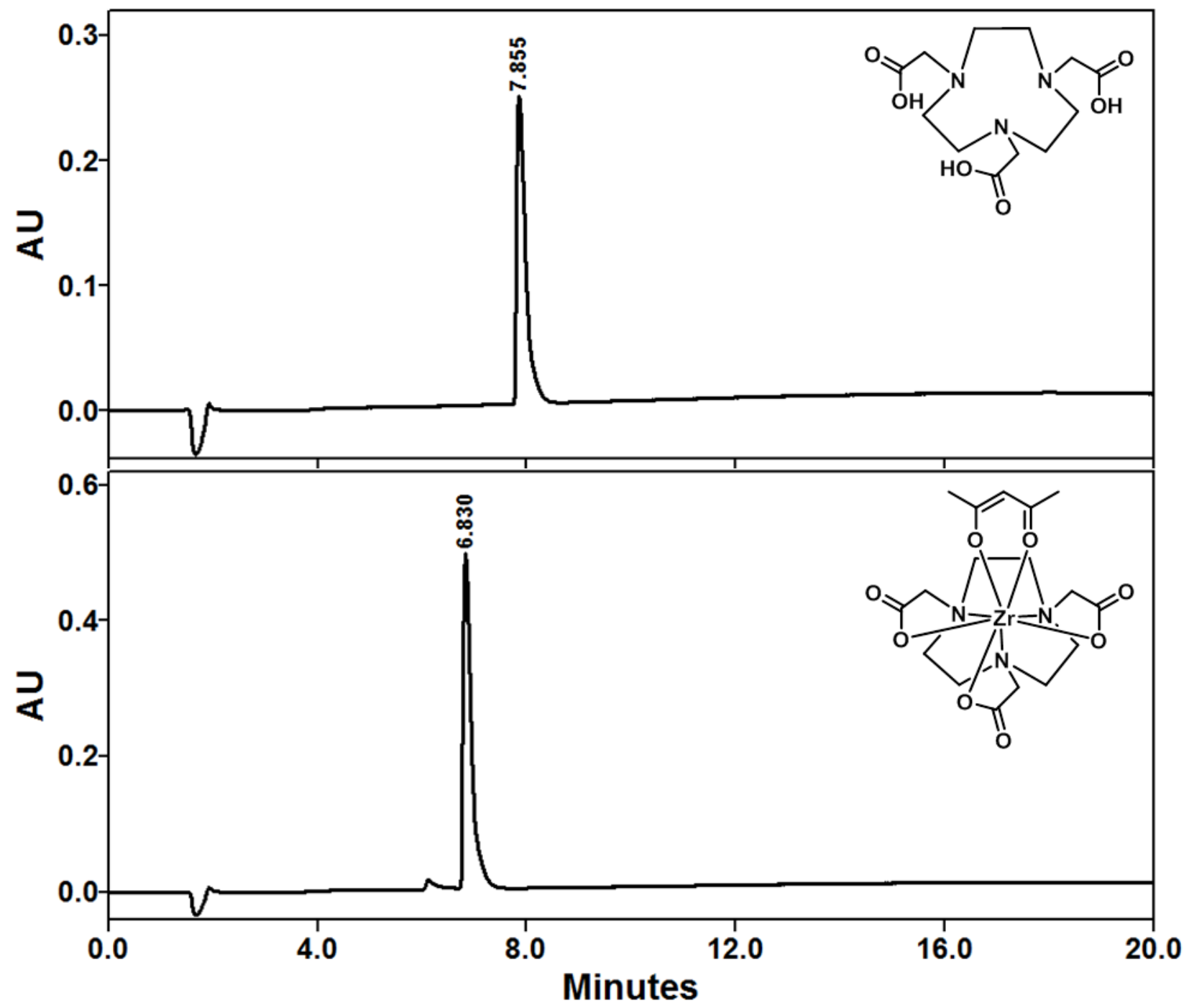

Figure S15. UV-HPLC chromatogram $(201 \mathrm{~nm})$ of NOTA ligand (top) and nonradioactive NatZr-NOTA-AcAC complex (bottom) 


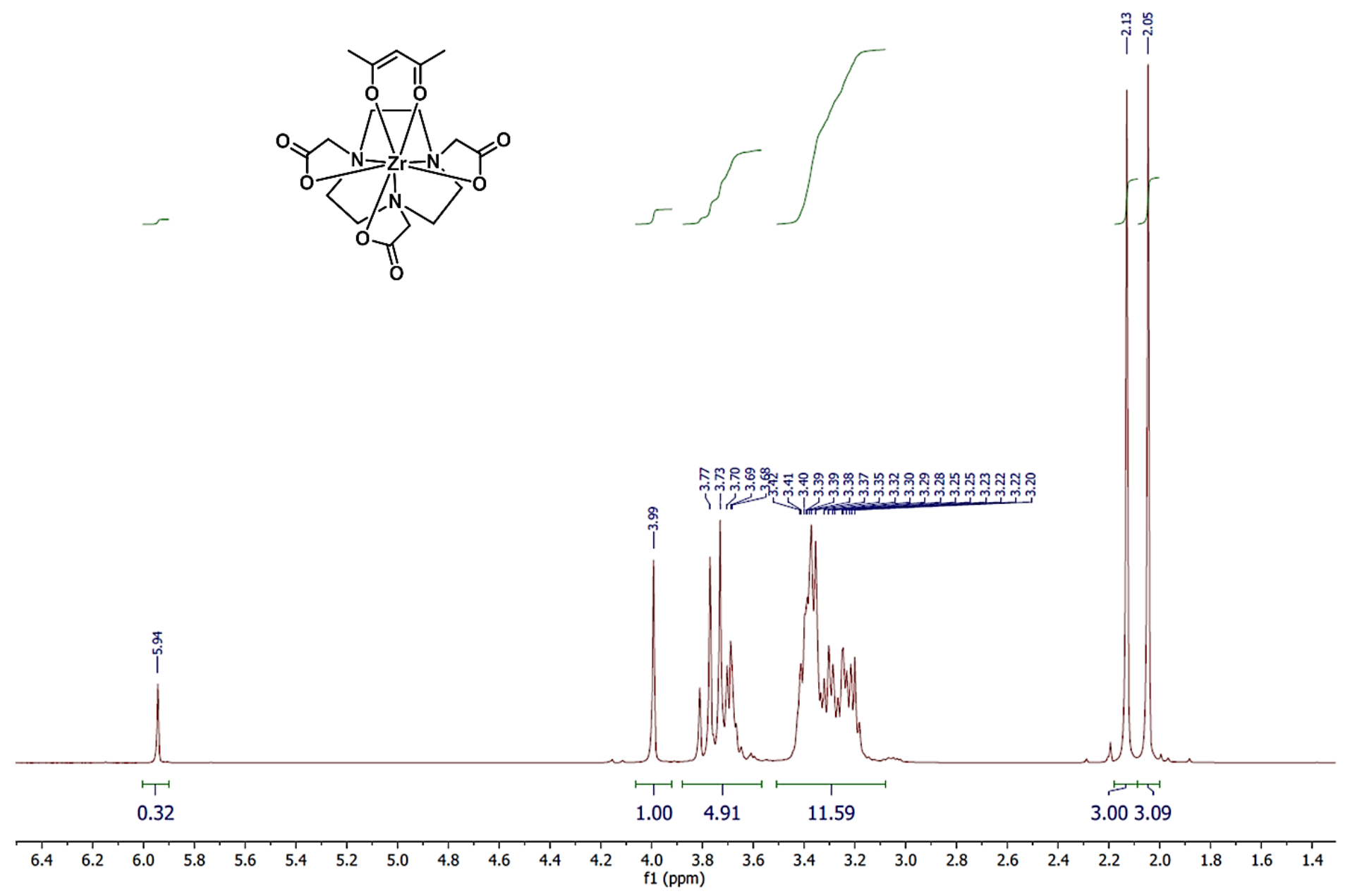

Figure S16. ${ }^{1} \mathrm{H}-\mathrm{NMR}$ spectrum $\left(\mathrm{D}_{2} \mathrm{O}-400 \mathrm{MHz}\right)$ of the $\mathrm{Zr}$-NOTA-AcAc complex 


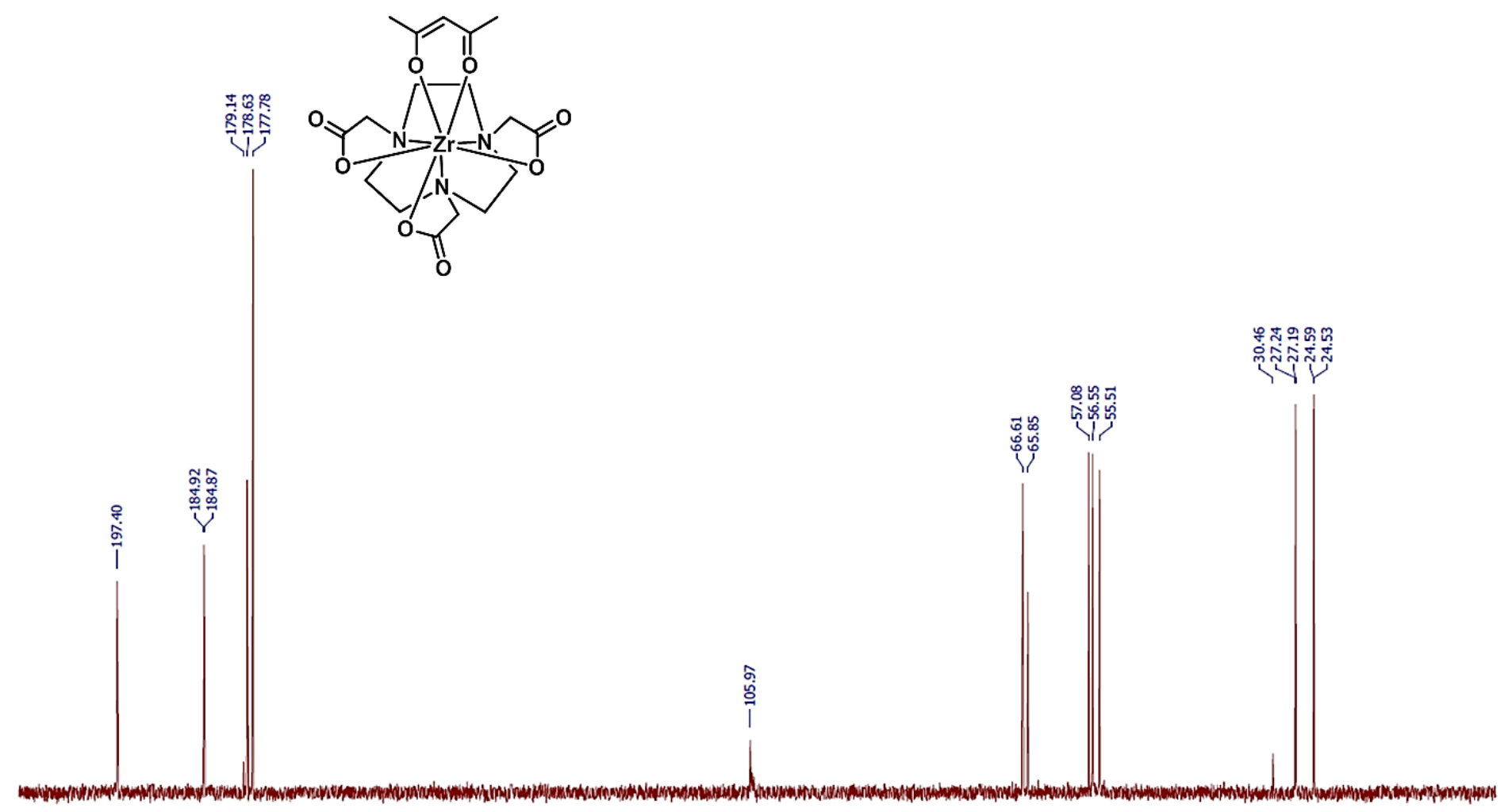

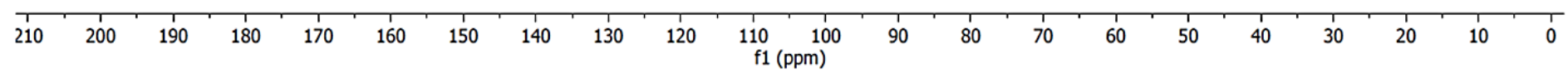

Figure $\mathrm{S} 17 .{ }^{13} \mathrm{C}-\mathrm{NMR}$ spectrum $\left(\mathrm{D}_{2} \mathrm{O}-101 \mathrm{MHz}\right)$ of the Zr-NOTA-AcAc complex 


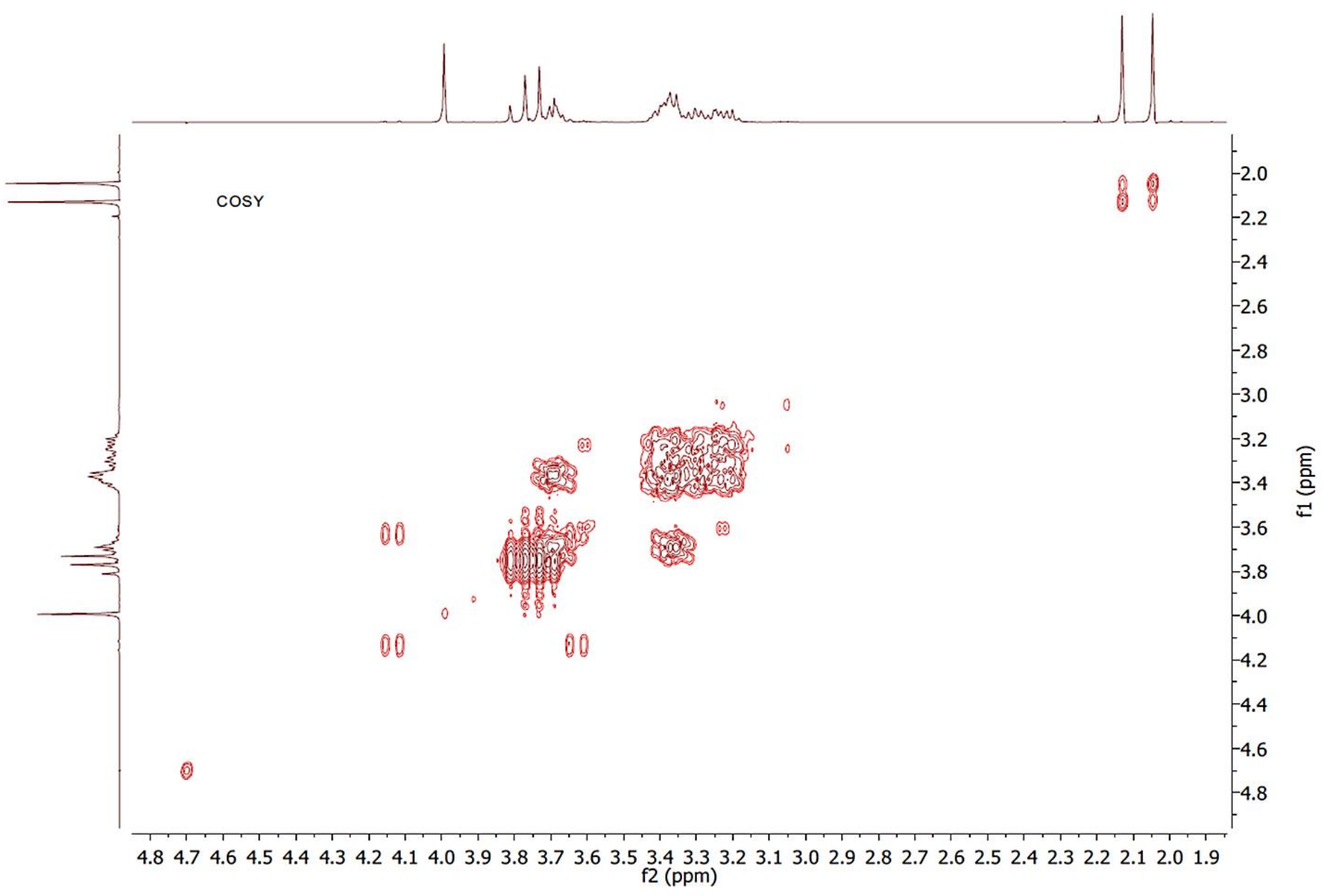

Figure S18. 2-D COSY NMR spectrum $\left(\mathrm{D}_{2} \mathrm{O}-400 \mathrm{MHz}\right)$ of the Zr-NOTA-AcAc complex 


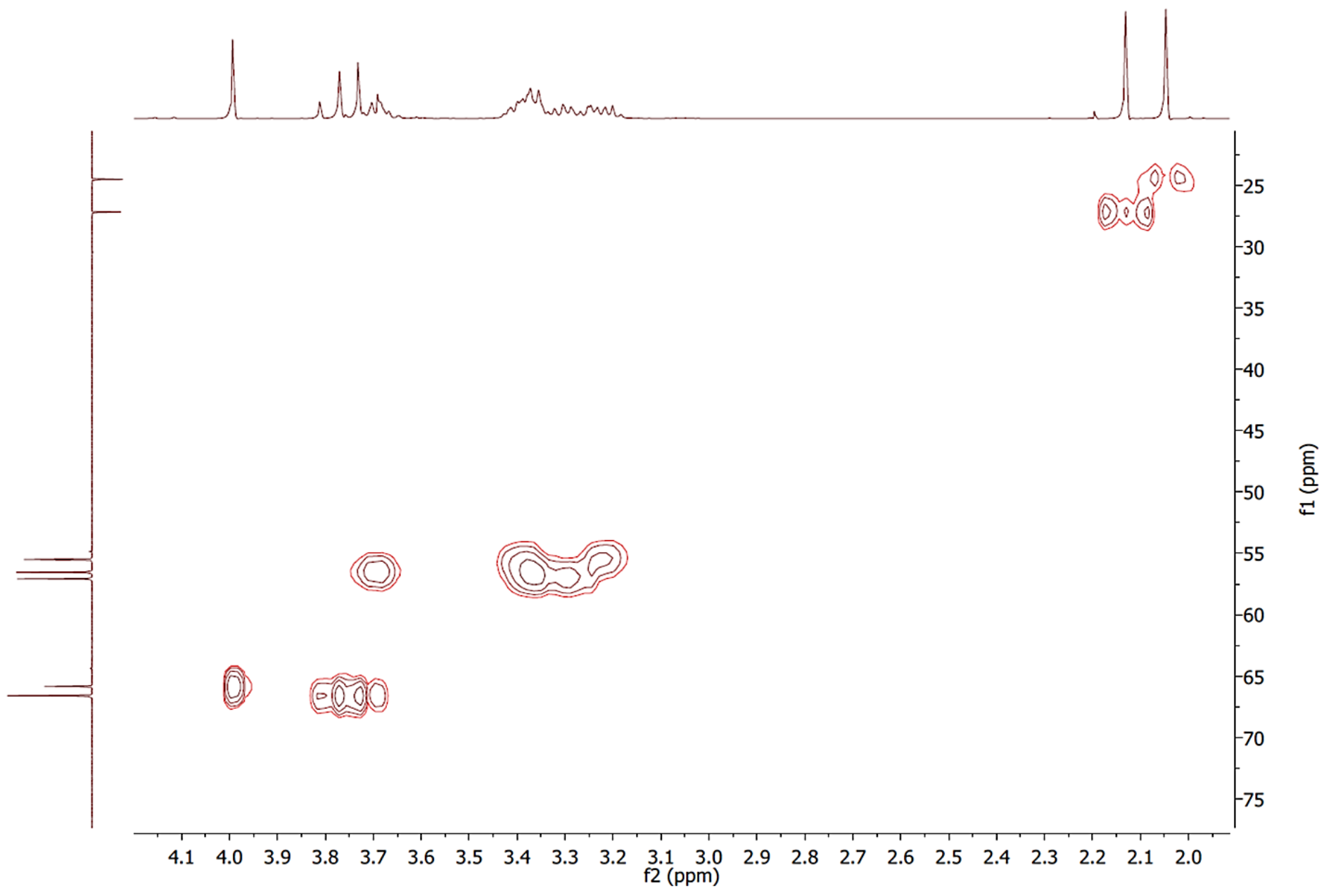

Figure S19. 2-D HMQC NMR spectrum $\left(\mathrm{D}_{2} \mathrm{O}-400 \mathrm{MHz}\right)$ of the Zr-NOTA-AcAc complex 


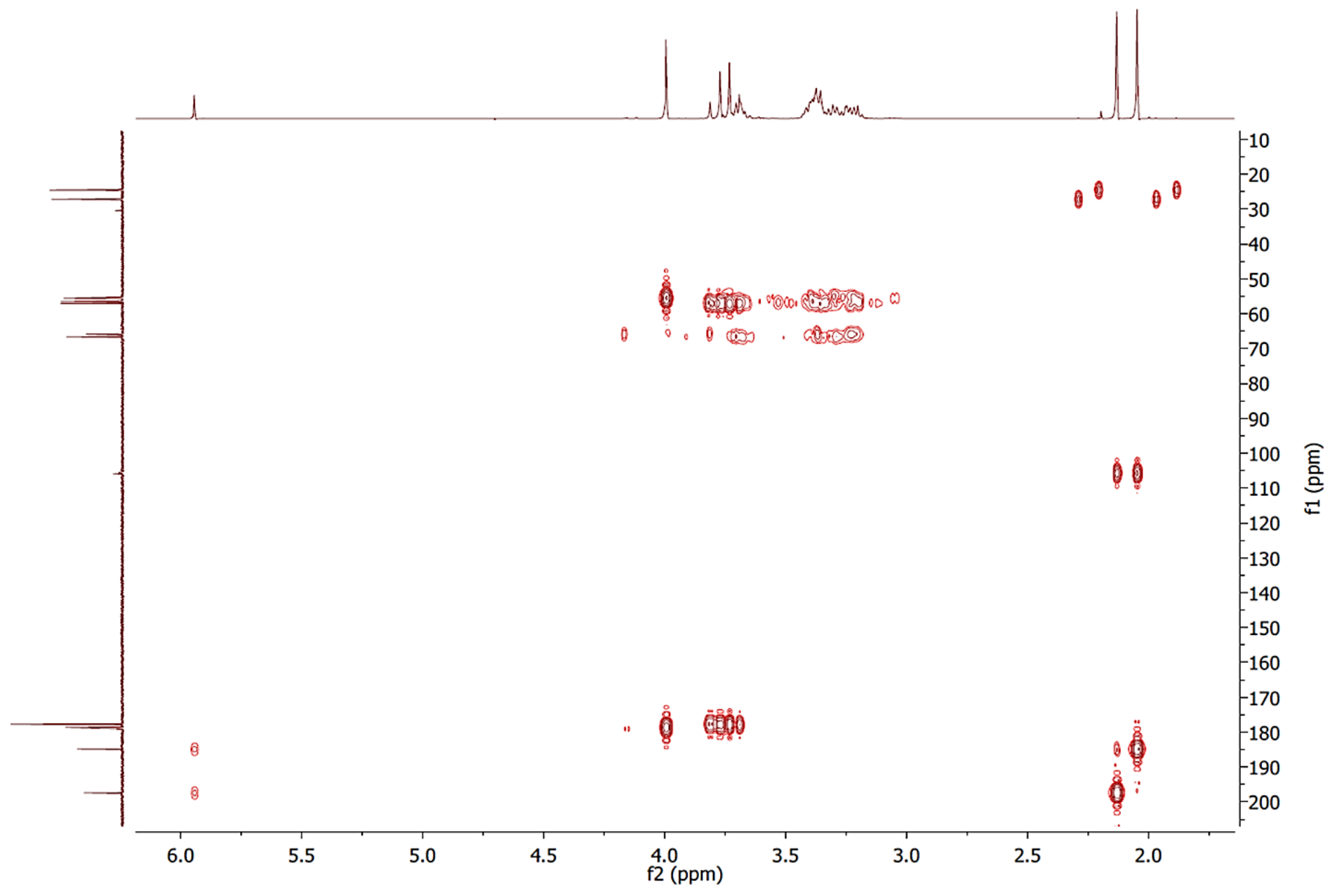

Figure S20. 2-D HMBC NMR spectrum $\left(\mathrm{D}_{2} \mathrm{O}-400 \mathrm{MHz}\right)$ of the Zr-NOTA-AcAc complex 


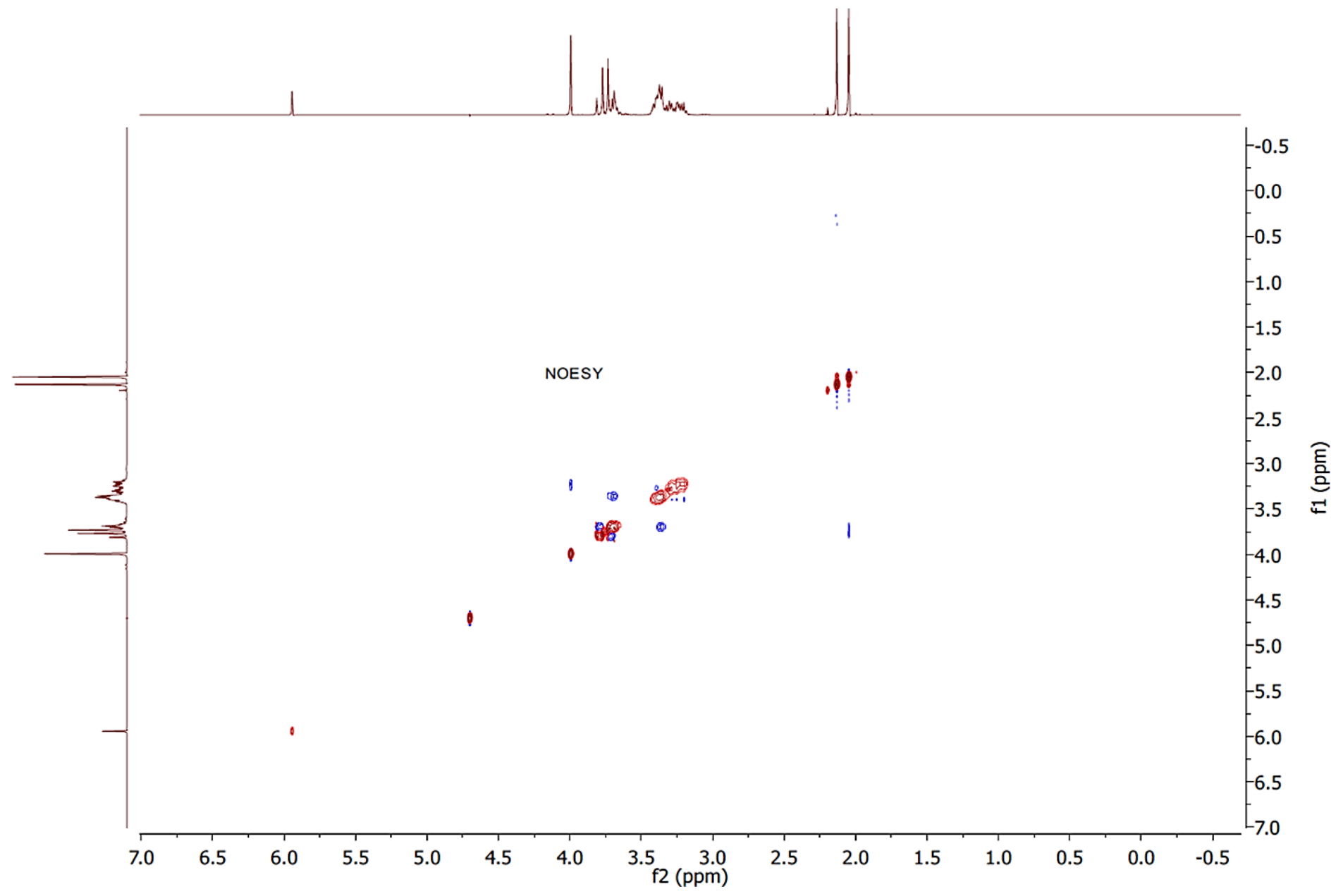

Figure S21. 2-D NOESY NMR spectrum $\left(\mathrm{D}_{2} \mathrm{O}-400 \mathrm{MHz}\right)$ of the Zr-NOTA-AcAc complex 


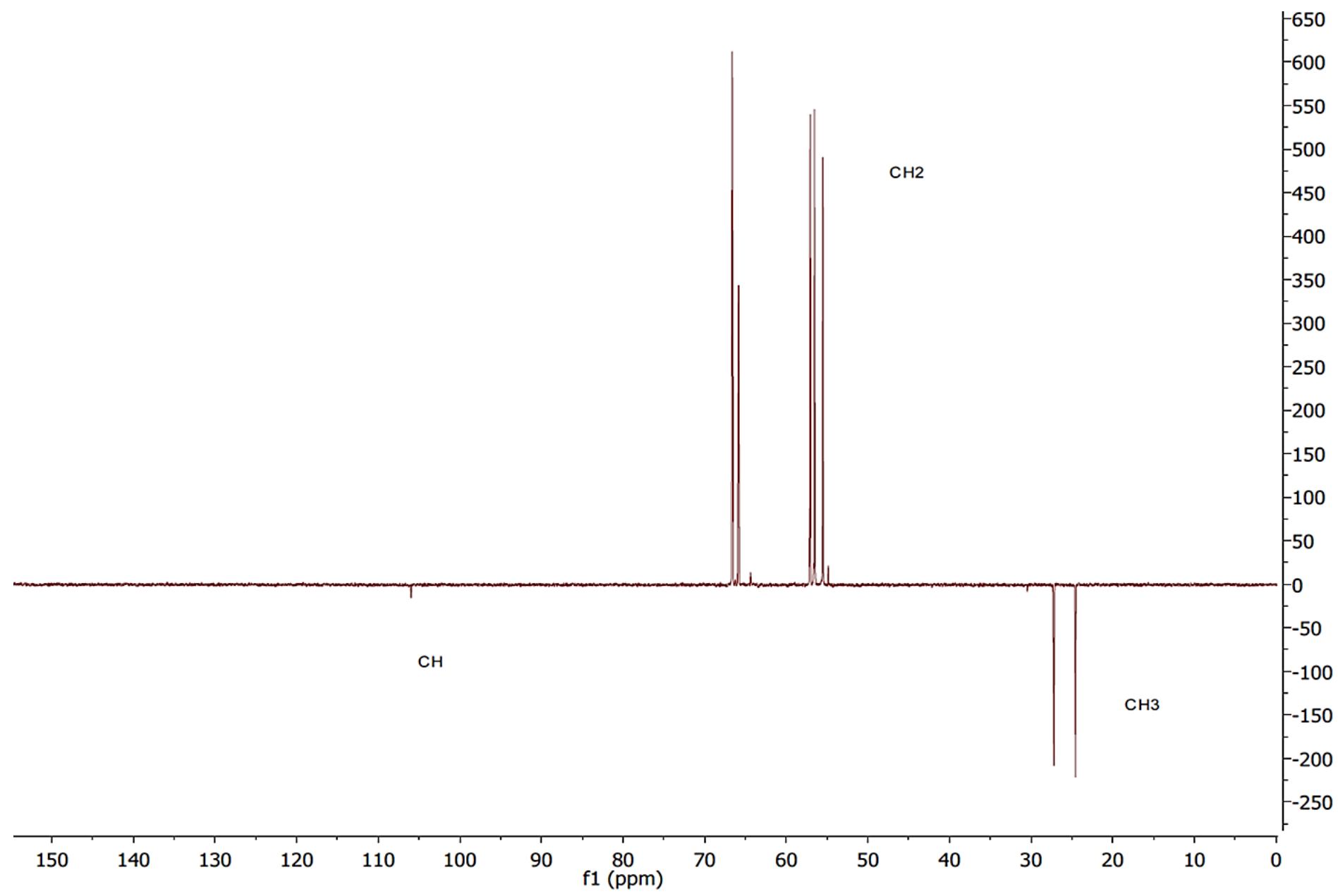

Figure S22. 2-D ${ }^{13} \mathrm{C}$ APT NMR spectrum $\left(\mathrm{D}_{2} \mathrm{O}-101 \mathrm{MHz}\right)$ of the $\mathrm{Zr}-\mathrm{NOTA}-\mathrm{AcAc}$ complex 


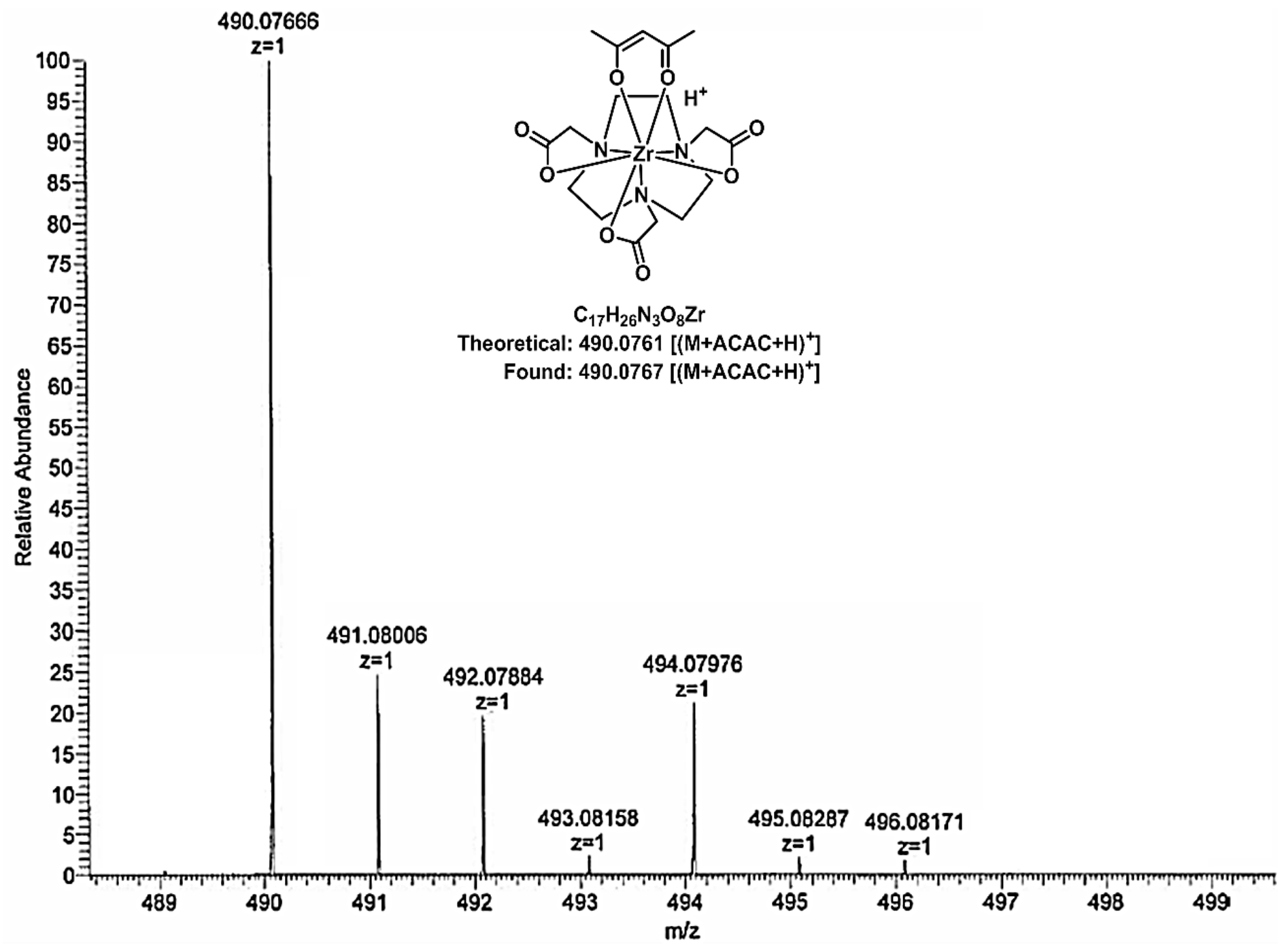

Figure S23. HR ESI FT-ICR MS (Positive mode) analysis of the Zr-NOTA-AcAc complex 


\section{Crystal Structure Analysis of $\mathrm{Zr}-\mathrm{TRITA},\left[\mathrm{Zr}\left(\mathrm{C}_{17} \mathrm{H}_{26} \mathrm{~N}_{4} \mathrm{O}_{8}\right)\right]-3.33 \mathrm{H}_{2} \mathrm{O}$}

\section{Crystallographic Experimental Details:}

Data Collection and Structure Solution. A clear colourless needle-like specimen of $\left[\mathrm{Zr}\left(\mathrm{C}_{17} \mathrm{H}_{26} \mathrm{~N}_{4} \mathrm{O}_{8}\right)\right]-3.33$ $\mathrm{H}_{2} \mathrm{O}$, approximate dimensions $0.010 \mathrm{~mm} \times 0.040 \mathrm{~mm} \times 0.470 \mathrm{~mm}$, was used for the X-ray crystallographic analysis. The X-ray intensity data were measured on a Bruker APEX CCD system equipped with a graphite monochromator and a $\operatorname{MoK}_{\alpha}$ sealed x-ray tube $(\lambda=0.71073 \AA)$. X-rays were provided by a fine-focus sealed x-ray tube operated at 50kV and 30mA.

The total exposure time was 18.65 hours. The frames were integrated with the Bruker SAINT Software ${ }^{1}$ package using a narrow-frame algorithm. The integration of the data using a monoclinic unit cell yielded a total of 61502 reflections to a maximum $\theta$ angle of $30.14^{\circ}(0.71 \AA$ resolution), of which 19202 were independent (average redundancy 3.203 , completeness $=99.4 \%, R_{\text {int }}=5.90 \%, R_{\text {sig }}=7.12 \%$ ) and $16822(87.61 \%)$ were greater than $2 \sigma\left(F^{2}\right)$. The final cell constants of $a=19.2657$ (17) $\AA, b=6.9888$ (6) $\AA, c=49.787$ (4) $\AA$, $\beta=99.7028$ $(11)^{\circ}$, volume $=6607.6(10) \AA^{3}$, are based upon the refinement of the XYZ-centroids of 9909 reflections above $20 \sigma(\mathrm{I})$ with $6.826^{\circ}<2 \theta<55.22^{\circ}$. Data were corrected for absorption effects using the multi-scan method $(\mathrm{SADABS})^{2}$. The ratio of minimum to maximum apparent transmission was 0.841 . The calculated minimum and maximum transmission coefficients (based on crystal size) are 0.776 and 0.994 .

The structure was solved and refined using the Bruker SHELXTL Software Package ${ }^{3}$, using the space group $\mathrm{Cc}$, with $\mathrm{Z}=12$ for the formula unit, $\left[\mathrm{Zr}\left(\mathrm{C}_{17} \mathrm{H}_{26} \mathrm{~N}_{4} \mathrm{O}_{8}\right)\right]-3.33 \mathrm{H}_{2} \mathrm{O}$ (3 formula units/asymmetric unit). The hydrogen atoms of the "TRITA" ligand were included in the structural model as fixed atoms (using idealized sp-hybridized geometry and $\mathrm{C}-\mathrm{H}$ bond lengths of $0.99 \AA \AA$ ) "riding" on their respective carbon atoms. The isotropic thermal parameters for all "TRITA" hydrogen atoms were fixed at values 1.2 times the equivalent isotropic thermal parameter of the carbon atom to which they are covalently bonded. The isotropic thermal parameters of the water hydrogen atoms were allowed to refine independently. The final anisotropic full-matrix least-squares refinement on $F^{2}$ with 981 variables converged at $R_{1}=3.96 \%$, for the observed data and $w R_{2}=7.93 \%$ for all data. The goodness-of-fit was 0.996 . The largest peak in the final difference electron density synthesis was 0.550 $\mathrm{e}^{-} / \AA^{3}$ and the largest hole was $-0.618 \mathrm{e}^{-} / \AA^{3}$ with an RMS deviation of $0.078 \mathrm{e}^{-} / \AA^{3}$. On the basis of the final model, the calculated density was $1.706 \mathrm{~g} / \mathrm{cm}^{3}$ and $\mathrm{F}(000), 3520 \mathrm{e}^{-}$. 
Refinement Details. Crystal data, data collection and structure refinement details are summarized in tables below.

Computing details. Data collection: Bruker APEX3 v2016.1-0; cell refinement: Bruker APEX3 v2016.1-0; data reduction: Bruker APEX2 v2014.11-0; program(s) used to solve structure: SHELXS97 (Sheldrick 2008); program(s) used to refine structure: SHELXL2014 (Sheldrick, 2014); molecular graphics: Bruker APEX2 v2014.11-0; software used to prepare material for publication: Bruker APEX2 v2014.11-01-6. 


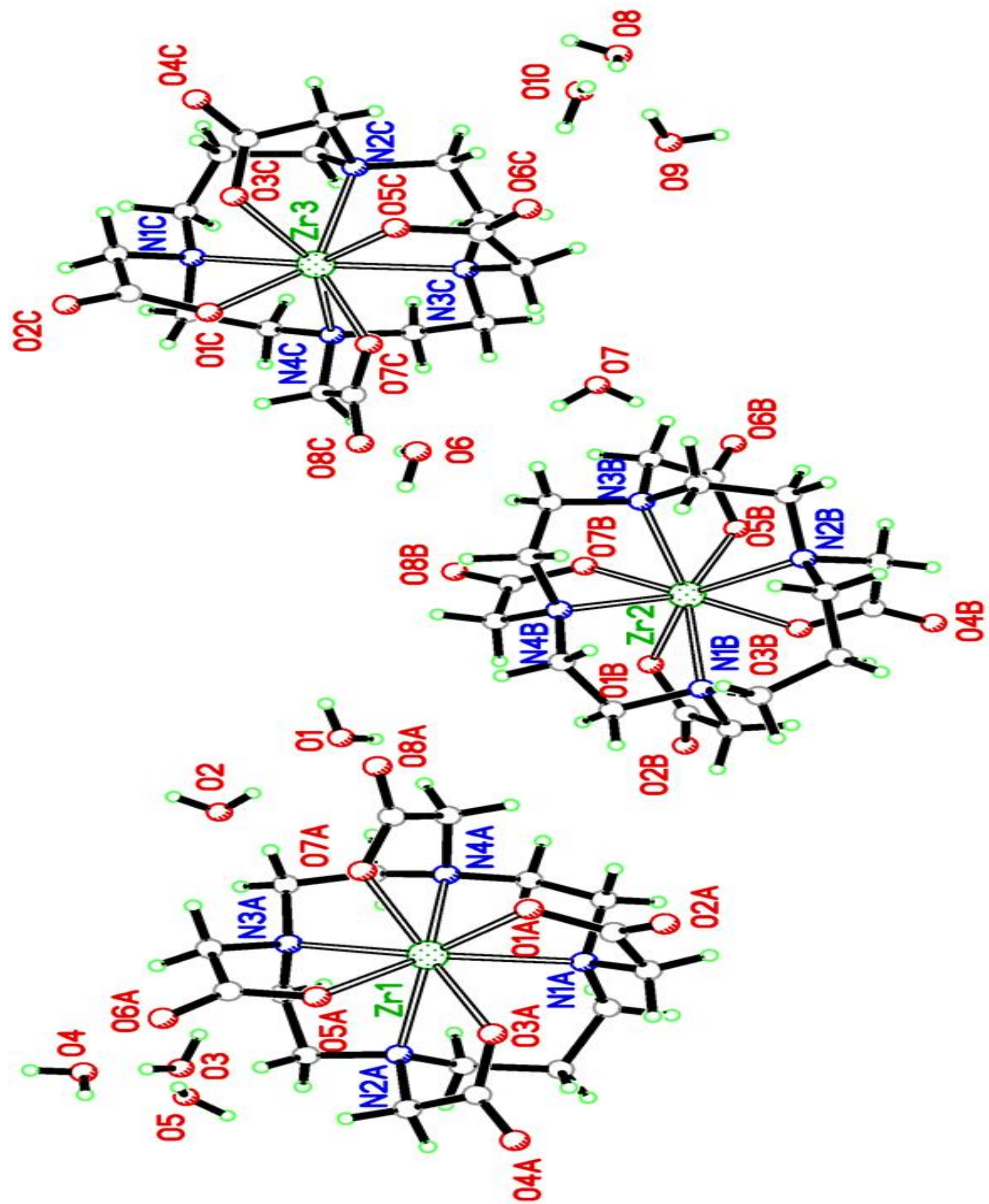

Figure S24. Plot for $\left[\mathrm{Zr}\left(\mathrm{C}_{17} \mathrm{H}_{26} \mathrm{~N}_{4} \mathrm{O}_{8}\right)\right]-3.33 \mathrm{H}_{2} \mathrm{O}$ showing the contents of the asymmetric unit. All atoms are represented by arbitrary-sized spheres and only selected atoms are labeled. 
(a)

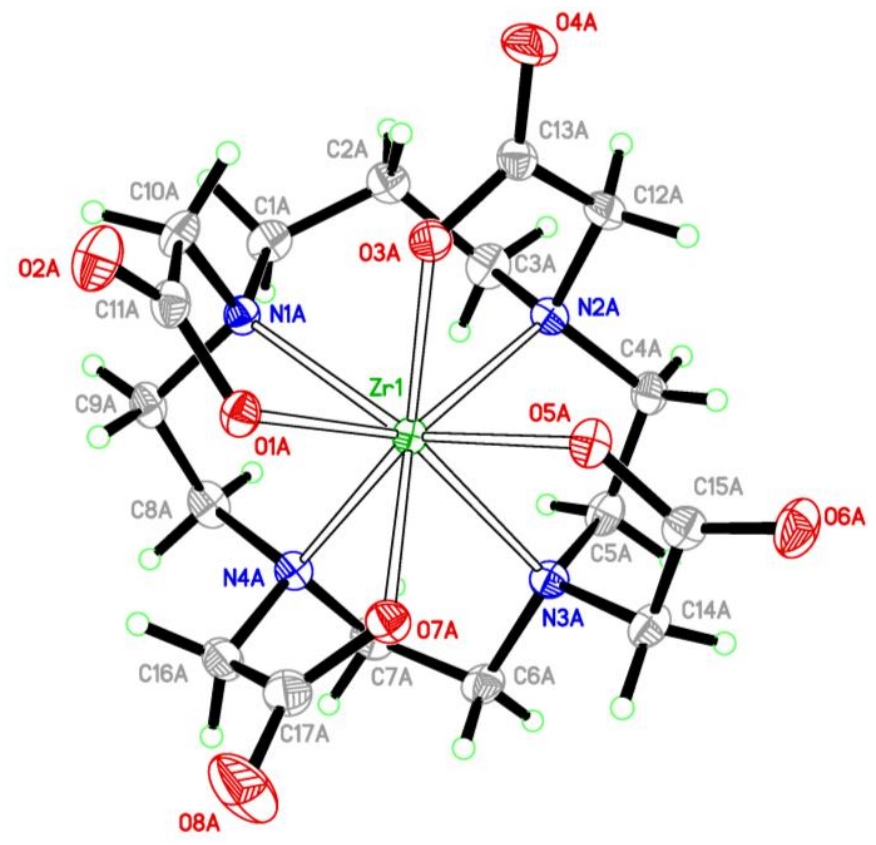

(b)

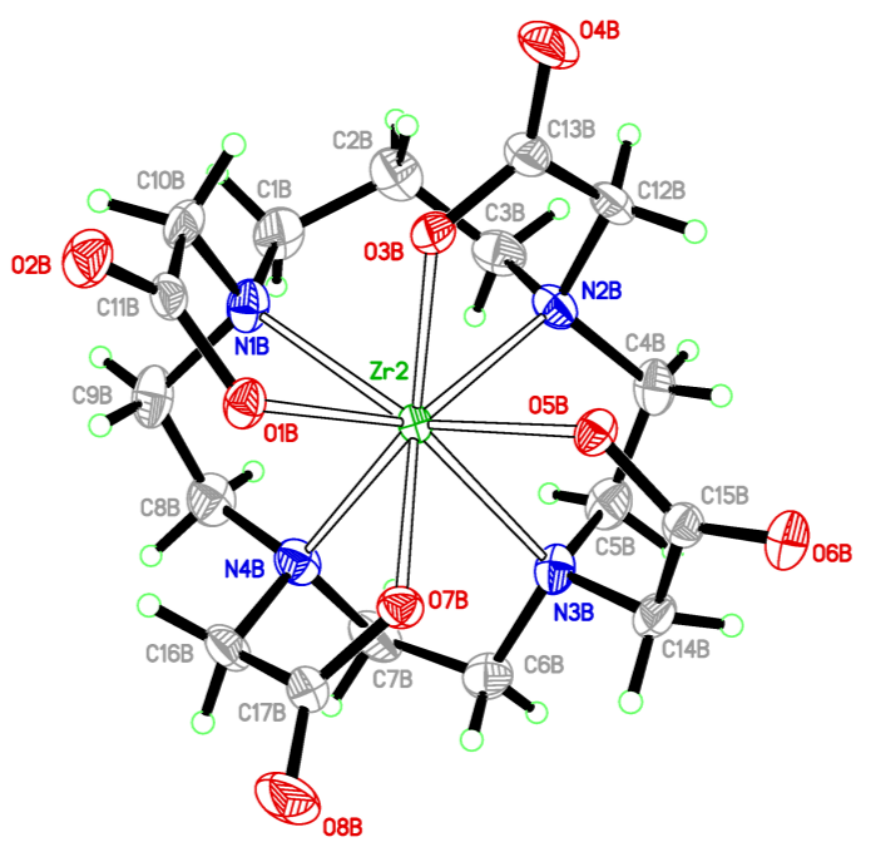

(C)

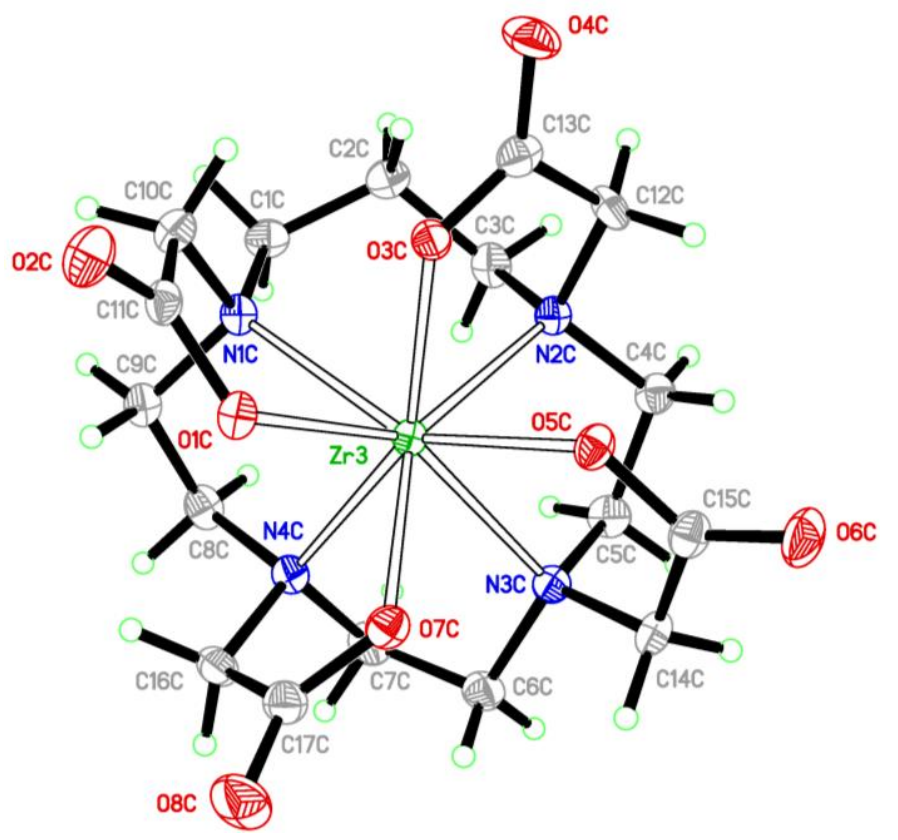

Figure S25. 50\% probability plots for the 3 crystallographically-independent $\left[\mathrm{Zr}\left(\mathrm{C}_{17} \mathrm{H}_{26} \mathrm{~N}_{4} \mathrm{O}_{8}\right)\right]$ molecules in the solid-state structure of $\left[\mathrm{Zr}\left(\mathrm{C}_{17} \mathrm{H}_{26} \mathrm{~N}_{4} \mathrm{O}_{8}\right)\right]-3.33 \mathrm{H}_{2} \mathrm{O}$ with hydrogen atoms represented by arbitrarysized spheres. 


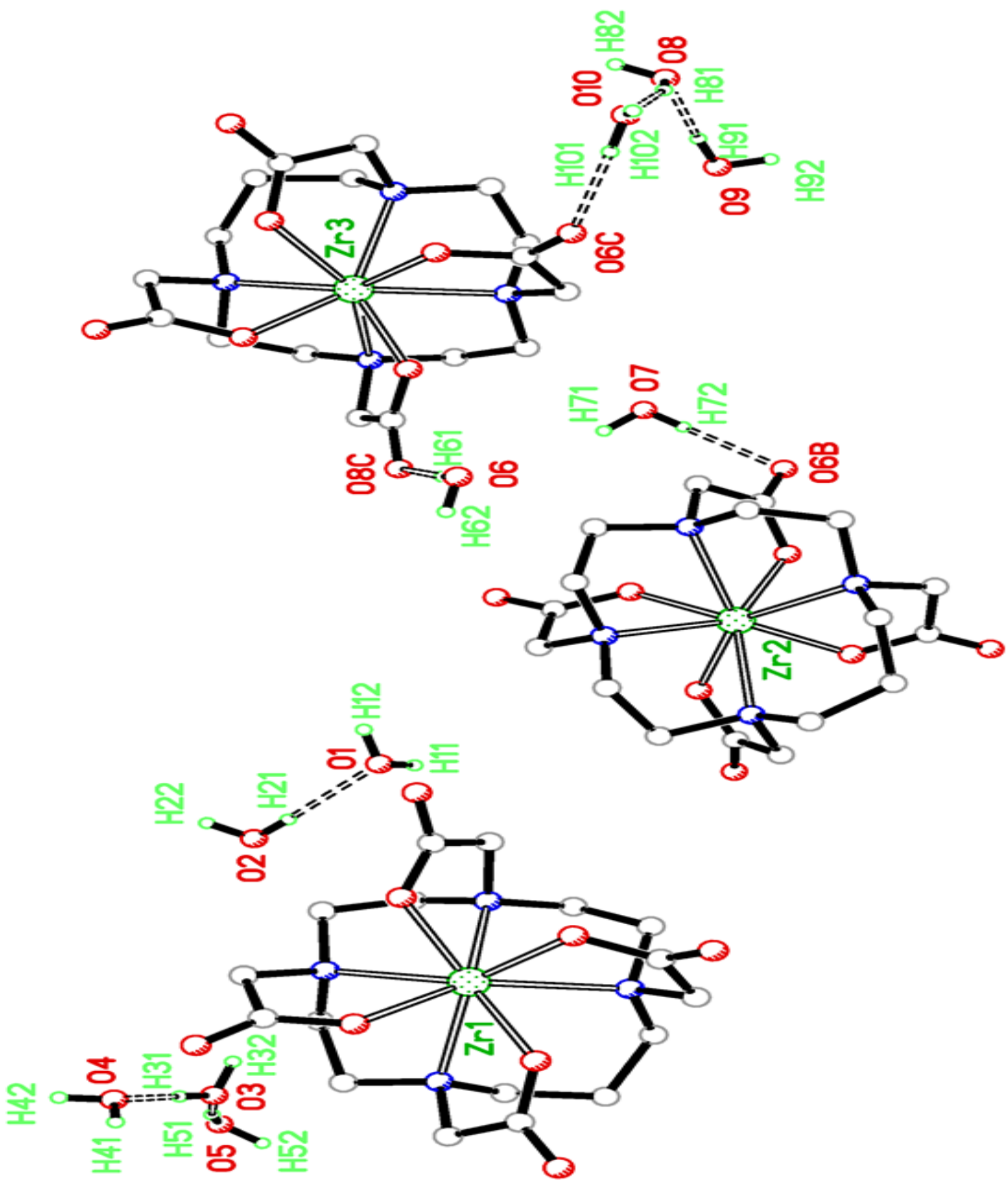

Figure S26. Plot showing the contents of the asymmetric unit for $\left[\mathrm{Zr}\left(\mathrm{C}_{17} \mathrm{H}_{26} \mathrm{~N}_{4} \mathrm{O}_{8}\right)\right]-3.33 \mathrm{H}_{2} \mathrm{O}$ with all atoms represented by arbitrary-sized spheres. Ligand hydrogen atoms have been omitted and hydrogen-bonding interactions involving the lattice water molecules with the 3 independent $\left[\mathrm{Zr}\left(\mathrm{C}_{17} \mathrm{H}_{26} \mathrm{~N}_{4} \mathrm{O}_{8}\right)\right]$ molecules are represented by dashed-open bonds. 


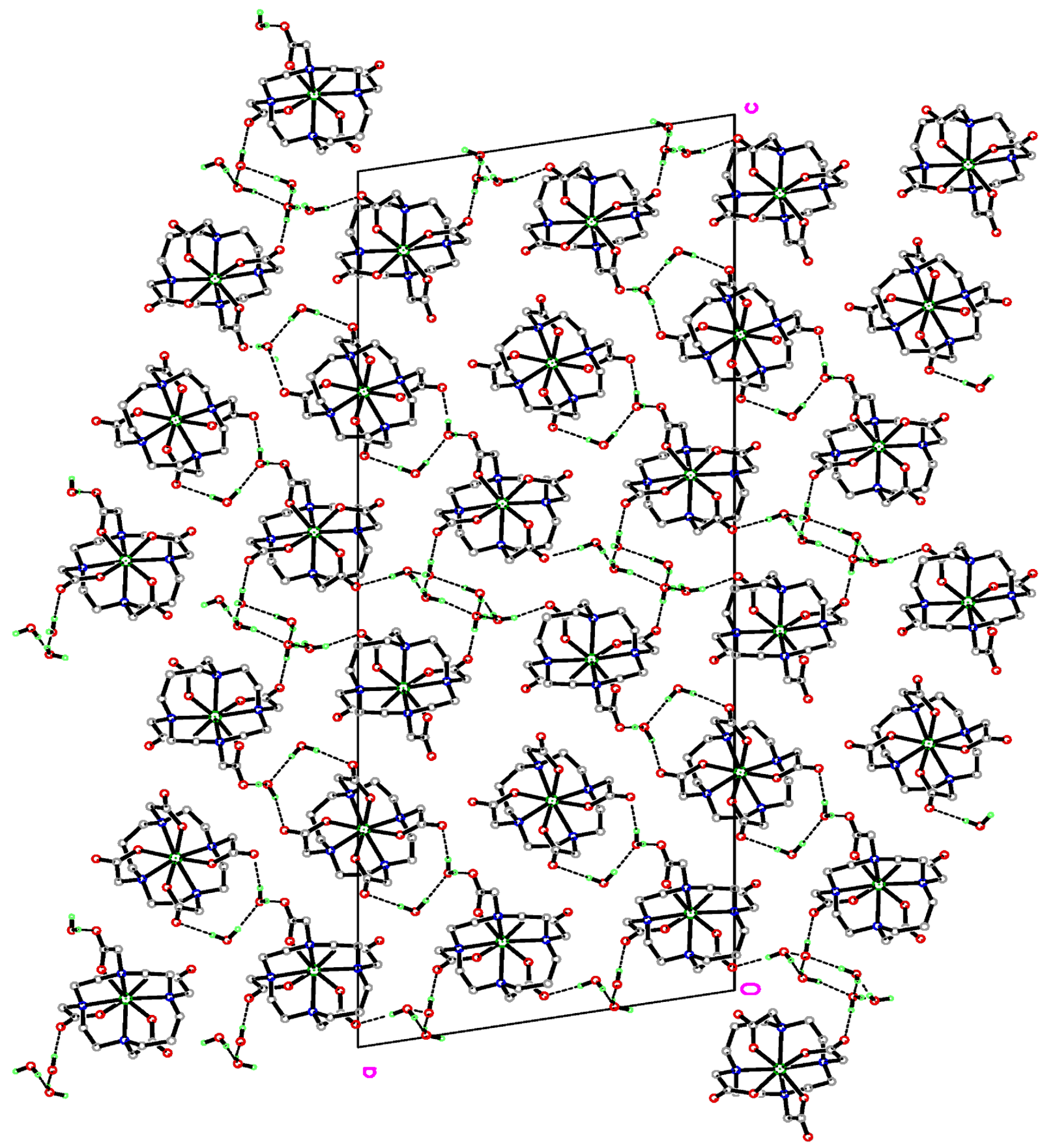

Figure S27. Packing plot of the solid-state structure of $\left[\mathrm{Zr}\left(\mathrm{C}_{17} \mathrm{H}_{26} \mathrm{~N}_{4} \mathrm{O}_{8}\right)\right]-3.33 \mathrm{H}_{2} \mathrm{O}$ viewed in projection down the $\vec{b}$ axis of the unit cell. All atoms are represented by dummy-sized spheres and ligand hydrogen atoms have been omitted for clarity. Hydrogen-bonding interactions involving the lattice water molecules are represented by dashed-solid bonds. 

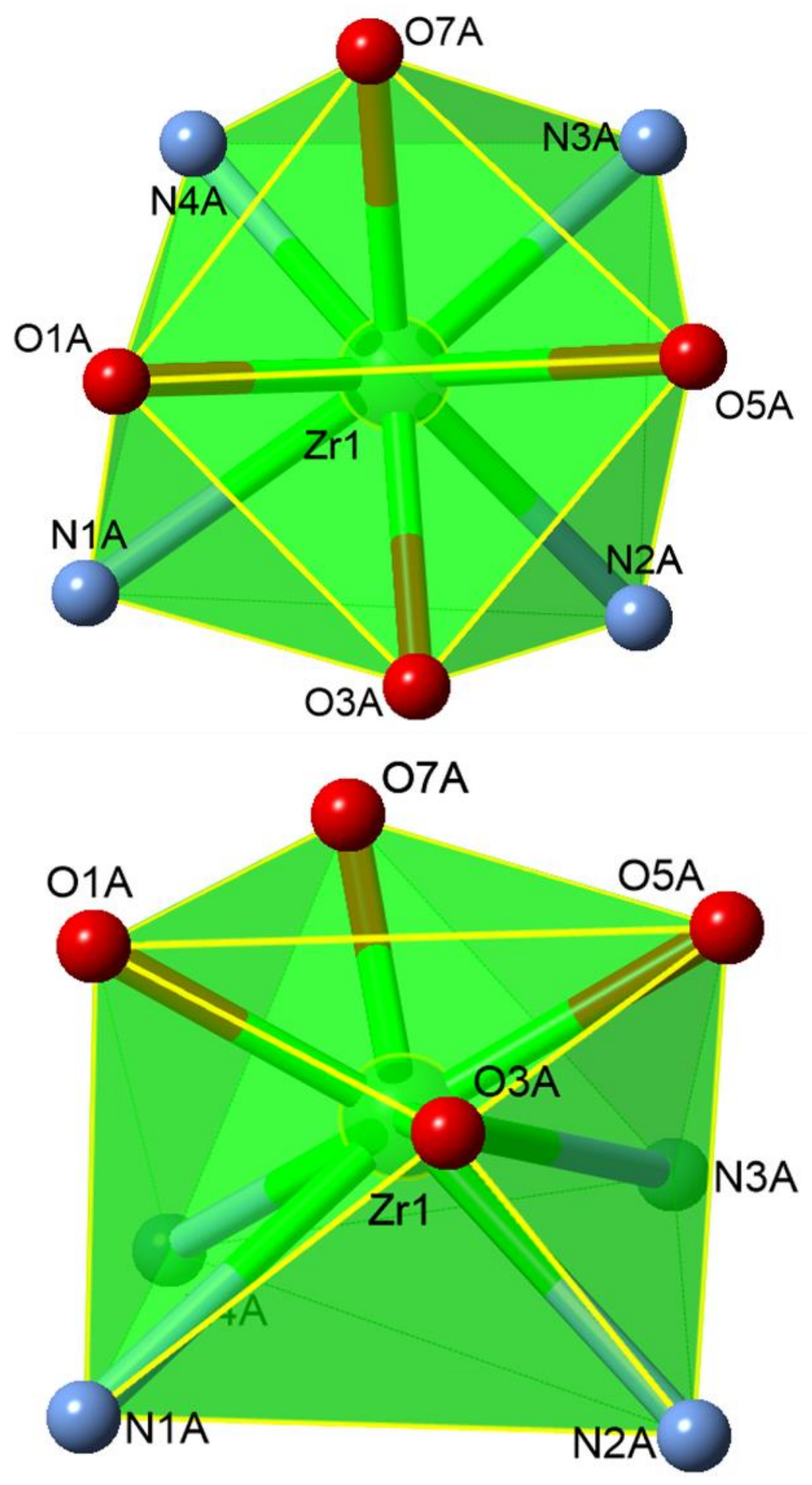

Figure S28. Molecule 1, top and side views showing the "distorted" square antiprism arrangement about Zr1. 

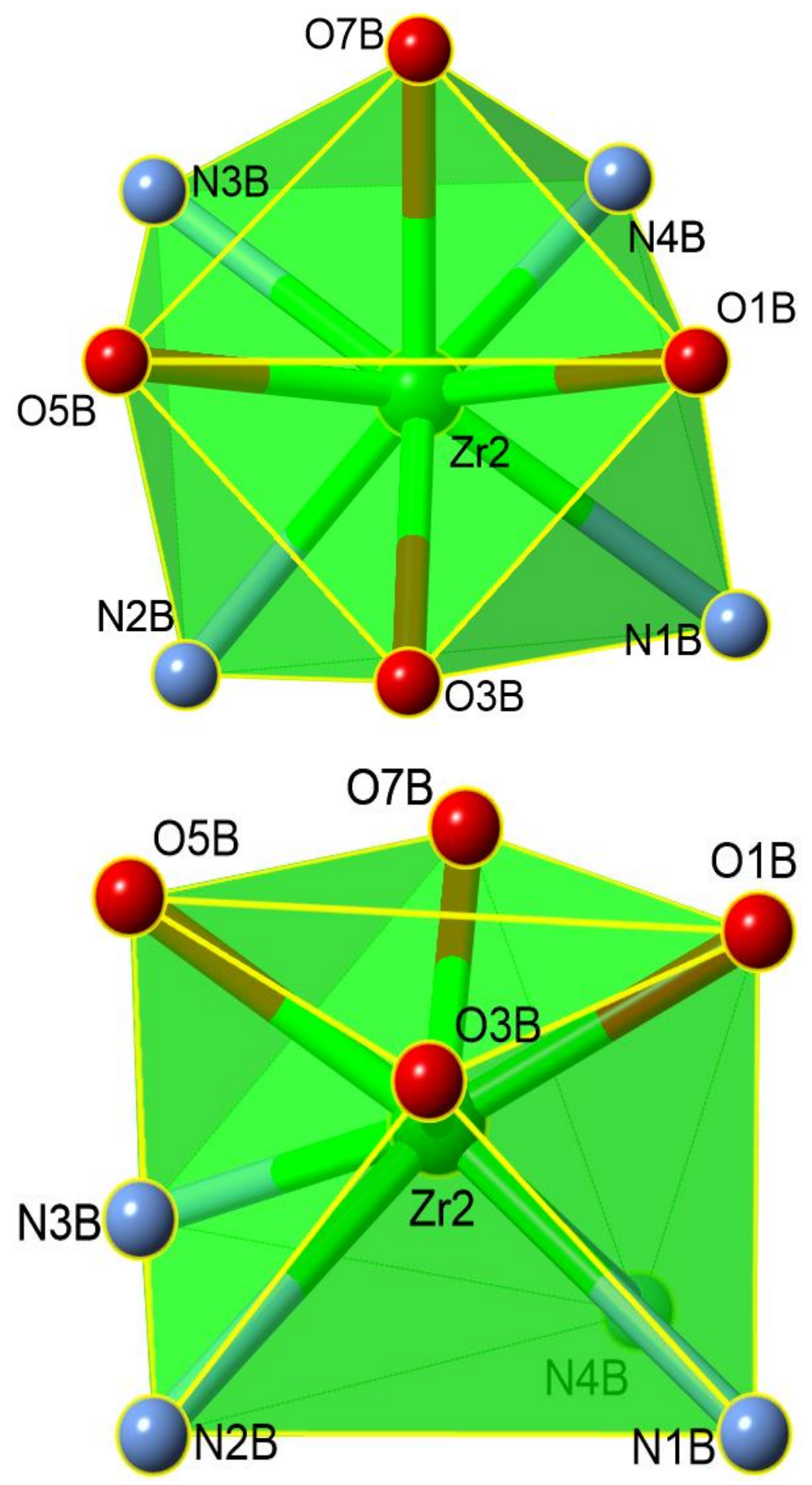

Figure S29. Molecule 2, top and side views showing the "distorted" square antiprism arrangement about Zr2. 

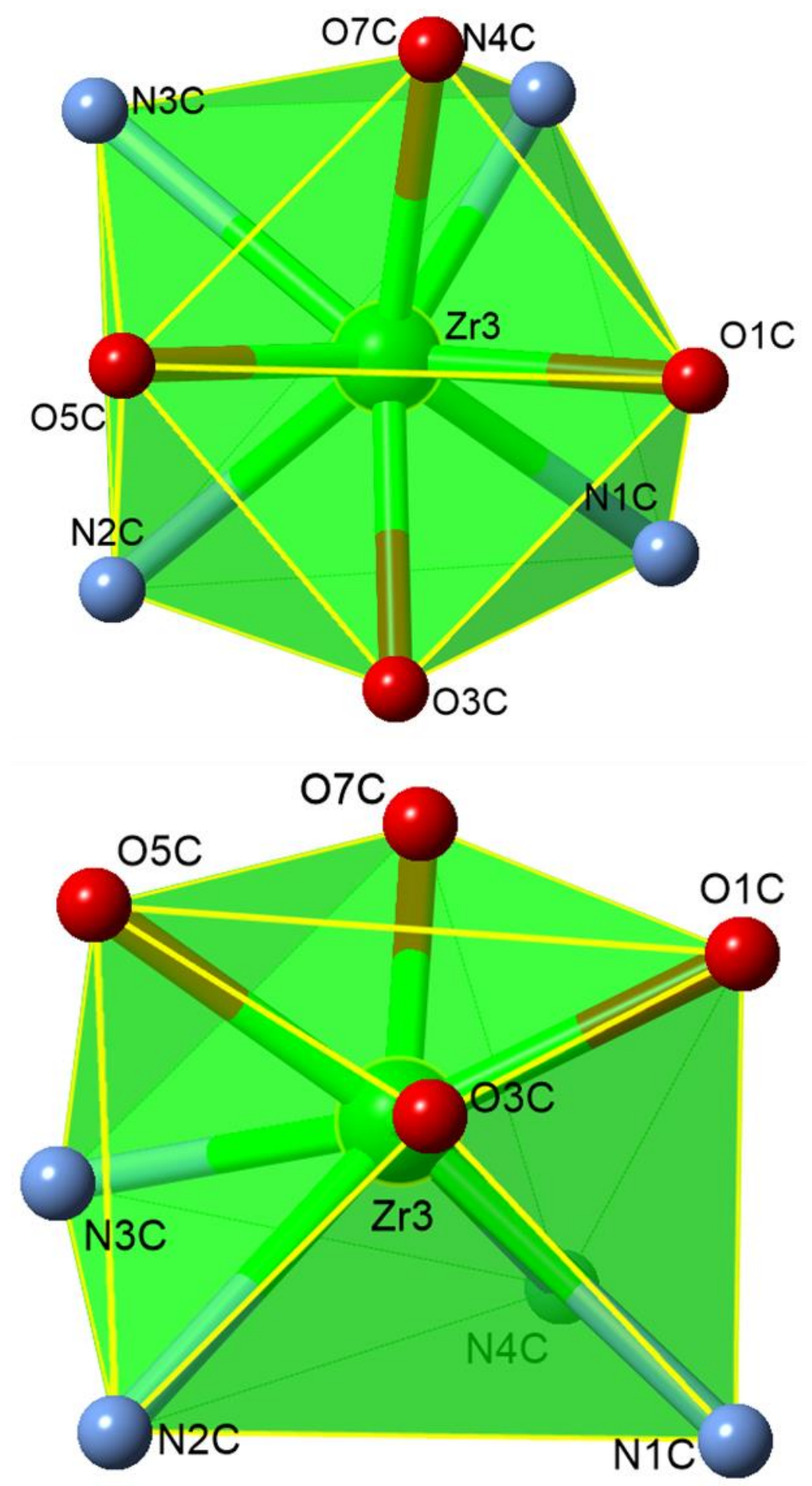

Figure S30. Molecule 3, top and side views showing the "distorted" square antiprism arrangement about Zr3. 
Table S1. Crystal structure data collection parameters

\section{Crystal data}

\begin{tabular}{|c|c|}
\hline $\mathrm{C}_{17} \mathrm{H}_{32.67} \mathrm{~N}_{4} \mathrm{O}_{11.33} \mathrm{Zr}$ & $F(000)=3520$ \\
\hline$M_{r}=565.69$ & $D_{\mathrm{x}}=1.706 \mathrm{Mg} \mathrm{m}^{-3}$ \\
\hline Monoclinic, Cc & Mo $K \alpha$ radiation, $\lambda=0.71073 \AA$ \\
\hline$a=19.2657$ (17) $\AA$ & Cell parameters from 9909 reflections \\
\hline$b=6.9888(6) \AA$ & $\theta=3.4-27.6^{\circ}$ \\
\hline$c=49.787(4) \AA$ & $\mu=0.57 \mathrm{~mm}^{-1}$ \\
\hline$\beta=99.7028(11)^{\circ}$ & $T=193 \mathrm{~K}$ \\
\hline$V=6607.6(10) \AA^{3}$ & Needle, colourless \\
\hline$Z=12$ & $0.47 \times 0.04 \times 0.01 \mathrm{~mm}$ \\
\hline
\end{tabular}

\section{Data collection}

\begin{tabular}{|l|l|}
\hline Bruker APEX CCD diffractometer & 19202 independent reflections \\
\hline Radiation source: sealed x-ray tube & 16822 reflections with $I>2 \square(I)$ \\
\hline Graphite monochromator & $R_{\text {int }}=0.059$ \\
\hline$\phi$ and $\omega$ scans & $\theta_{\max }=30.1^{\circ}, \theta_{\min }=3.4^{\circ}$ \\
\hline $\begin{array}{l}\text { Absorption correction: multi-scan Data were } \\
\text { corrected for scaling and absorption effects using } \\
\text { the multi-scan technique (SADABS). The ratio of } \\
\text { minimum to maximum apparent transmission was } \\
0.841 . \text { The calculated minimum and maximum } \\
\text { transmission coefficients (based on crystal size) are } \\
0.776 \text { and } 0.994 .\end{array}$ & $h=-27 \rightarrow 27$ \\
\hline $\begin{array}{l}T_{\text {min }}=0.627, T_{\text {max }}=0.746 \\
61502 \text { measured reflections }\end{array}$ & $k=-9 \rightarrow 9$ \\
\hline
\end{tabular}




\section{Refinement}

\begin{tabular}{|c|c|}
\hline Refinement on $F^{2}$ & Secondary atom site location: difference Fourier map \\
\hline Least-squares matrix: full & Hydrogen site location: mixed \\
\hline$R\left[F^{2}>2 \sigma\left(F^{2}\right)\right]=0.040$ & $\begin{array}{l}\mathrm{H} \text { atoms treated by a mixture of independent and } \\
\text { constrained refinement }\end{array}$ \\
\hline$w R\left(F^{2}\right)=0.079$ & $\begin{array}{l}w=1 /\left[\sigma^{2}\left(F_{0}^{2}\right)+(0.0304 P)^{2}\right] \\
\text { where } P=\left(F_{0}^{2}+2 F_{c}^{2}\right) / 3\end{array}$ \\
\hline$S=1.00$ & $(\Delta / \sigma)_{\max }=0.003$ \\
\hline 19202 reflections & $\Delta\rangle_{\max }=0.55 \mathrm{e}^{-3}$ \\
\hline 981 parameters & 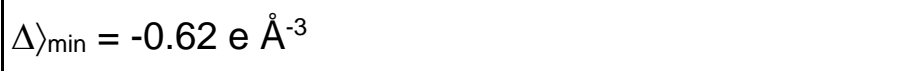 \\
\hline 32 restraints & $\begin{array}{l}\text { Absolute structure: Flack x determined using } 6966 \\
\text { quotients }[(\mathrm{I}+)-(\mathrm{I}-)] /[(\mathrm{I}+)+(\mathrm{I}-)] \quad \text { (Parsons, Flack and } \\
\text { Wagner, Acta Cryst. B69 (2013) 249-259) }\end{array}$ \\
\hline $\begin{array}{l}\text { Primary atom site location: structure-invariant direct } \\
\text { methods }\end{array}$ & Absolute structure parameter: $0.016(16)$ \\
\hline
\end{tabular}

\section{Special details}

Geometry. All esds (except the esd in the dihedral angle between two I.s. planes) are estimated using the full covariance matrix. The cell esds are taken into account individually in the estimation of esds in distances, angles and torsion angles; correlations between esds in cell parameters are only used when they are defined by crystal symmetry. An approximate (isotropic) treatment of cell esds is used for estimating esds involving I.s. planes. 
Table S2. Fractional atomic coordinates and isotropic or equivalent isotropic displacement parameters $\left(\AA^{2}\right)$

\begin{tabular}{|c|c|c|c|c|}
\hline & $x$ & $y$ & $z$ & $U_{\text {iso }}{ }^{*} / U_{\text {eq }}$ \\
\hline Zr1 & $0.87976(2)$ & $1.48693(5)$ & $0.90232(2)$ & 0.01309 (8) \\
\hline O1A & $0.94954(16)$ & $1.6045(4)$ & $0.87864(6)$ & $0.0184(6)$ \\
\hline $\mathrm{O} 2 \mathrm{~A}$ & $1.05440(18)$ & $1.7422(4)$ & $0.88036(7)$ & $0.0288(8)$ \\
\hline O3A & 0.94959 (16) & $1.6617(4)$ & $0.93078(6)$ & $0.0193(6)$ \\
\hline O4A & 0.99029 (18) & $1.7775(5)$ & 0.97191 (7) & $0.0293(8)$ \\
\hline O5A & $0.81246(16)$ & $1.6897(4)$ & $0.91621(6)$ & $0.0195(6)$ \\
\hline $\mathrm{O} 6 \mathrm{~A}$ & $0.70833(18)$ & 1.7928 (5) & $0.92409(7)$ & 0.0298 (8) \\
\hline O7A & $0.81553(16)$ & $1.5623(4)$ & $0.86471(6)$ & $0.0198(6)$ \\
\hline O8A & 0.8047 (3) & 1.5478 (6) & $0.81969(8)$ & 0.0492 (12) \\
\hline N1A & 0.99584 (19) & $1.3074(5)$ & $0.90816(7)$ & $0.0167(7)$ \\
\hline N2A & 0.87506 (19) & $1.3835(5)$ & 0.94934 (7) & $0.0164(7)$ \\
\hline N3A & $0.76393(19)$ & $1.3369(5)$ & 0.90257 (7) & $0.0169(7)$ \\
\hline N4A & $0.86712(19)$ & $1.2041(5)$ & $0.87279(7)$ & $0.0169(7)$ \\
\hline C1A & $1.0215(2)$ & $1.1799(6)$ & $0.93188(9)$ & $0.0218(9)$ \\
\hline $\mathrm{H} 1 \mathrm{~A}$ & 1.0736 & 1.1759 & 0.9346 & $0.026^{*}$ \\
\hline H1B & 1.0042 & 1.0486 & 0.9273 & $0.026^{*}$ \\
\hline C2A & 0.9994 (2) & $1.2385(6)$ & 0.95851 (9) & 0.0217 (9) \\
\hline $\mathrm{H} 2 \mathrm{~A}$ & 1.0262 & 1.1613 & 0.9734 & $0.026^{*}$ \\
\hline $\mathrm{H} 2 \mathrm{~B}$ & 1.0122 & 1.3743 & 0.9622 & $0.026^{*}$ \\
\hline C3A & $0.9217(2)$ & $1.2142(6)$ & $0.95891(9)$ & $0.0231(10)$ \\
\hline $\mathrm{H} 3 \mathrm{~A}$ & 0.9157 & 1.1832 & 0.9778 & $0.028^{*}$ \\
\hline $\mathrm{H} 3 \mathrm{~B}$ & 0.9048 & 1.1028 & 0.9474 & $0.028^{*}$ \\
\hline C4A & $0.8013(2)$ & $1.3337(6)$ & $0.95242(9)$ & 0.0206 (9) \\
\hline $\mathrm{H} 4 \mathrm{~A}$ & 0.7747 & 1.4524 & 0.9545 & $0.025^{\star}$ \\
\hline H4B & 0.8021 & 1.2556 & 0.9691 & $0.025^{\star}$ \\
\hline C5A & $0.7651(2)$ & $1.2244(7)$ & $0.92816(9)$ & $0.0215(9)$ \\
\hline H5A & 0.7900 & 1.1018 & 0.9268 & $0.026^{*}$ \\
\hline
\end{tabular}




\begin{tabular}{|c|c|c|c|c|}
\hline H5B & 0.7163 & 1.1948 & 0.9305 & $0.026^{*}$ \\
\hline C6A & $0.7427(2)$ & $1.2061(7)$ & $0.87889(9)$ & $0.0220(9)$ \\
\hline $\mathrm{H} 6 \mathrm{~A}$ & 0.7030 & 1.1243 & 0.8821 & $0.026^{*}$ \\
\hline $\mathrm{H} 6 \mathrm{~B}$ & 0.7273 & 1.2818 & 0.8621 & $0.026^{*}$ \\
\hline C7A & $0.8044(3)$ & $1.0849(6)$ & $0.87558(10)$ & $0.0230(10)$ \\
\hline $\mathrm{H} 7 \mathrm{~A}$ & 0.7919 & 1.0035 & 0.8592 & $0.028^{*}$ \\
\hline H7B & 0.8161 & 0.9998 & 0.8916 & $0.028^{*}$ \\
\hline C8A & $0.9300(3)$ & $1.0753(6)$ & $0.87712(9)$ & $0.0219(10)$ \\
\hline $\mathrm{H} 8 \mathrm{~A}$ & 0.9275 & 0.9886 & 0.8926 & $0.026^{*}$ \\
\hline H8B & 0.9304 & 0.9960 & 0.8607 & $0.026^{*}$ \\
\hline C9A & $0.9959(2)$ & $1.1913(6)$ & $0.88279(9)$ & $0.0205(9)$ \\
\hline H9A & 0.9987 & 1.2774 & 0.8672 & $0.025^{\star}$ \\
\hline H9B & 1.0374 & 1.1057 & 0.8852 & $0.025^{\star}$ \\
\hline C10A & $1.0485(2)$ & $1.4633(6)$ & $0.90717(10)$ & $0.0206(10)$ \\
\hline $\mathrm{H} 10 \mathrm{~A}$ & 1.0912 & 1.4099 & 0.9013 & $0.025^{\star}$ \\
\hline $\mathrm{H} 10 \mathrm{~B}$ & 1.0624 & 1.5194 & 0.9256 & $0.025^{\star}$ \\
\hline C11A & $1.0172(2)$ & $1.6172(6)$ & $0.88738(9)$ & $0.0200(9)$ \\
\hline C12A & $0.8985(3)$ & $1.5472(7)$ & $0.96777(9)$ & $0.0209(10)$ \\
\hline $\mathrm{H} 12 \mathrm{~A}$ & 0.9202 & 1.4976 & 0.9859 & $0.025^{\star}$ \\
\hline $\mathrm{H} 12 \mathrm{~B}$ & 0.8570 & 1.6249 & 0.9702 & $0.025^{\star}$ \\
\hline C13A & $0.9508(2)$ & $1.6722(6)$ & $0.95666(8)$ & $0.0182(8)$ \\
\hline C14A & $0.7121(3)$ & $1.4947(6)$ & $0.90166(10)$ & $0.0209(10)$ \\
\hline $\mathrm{H} 14 \mathrm{~A}$ & 0.6729 & 1.4531 & 0.9108 & $0.025^{\star}$ \\
\hline $\mathrm{H} 14 \mathrm{~B}$ & 0.6923 & 1.5237 & 0.8824 & $0.025^{\star}$ \\
\hline C15A & $0.7445(2)$ & $1.6723(6)$ & $0.91518(9)$ & $0.0193(9)$ \\
\hline C16A & $0.8585(3)$ & $1.2848(7)$ & $0.84484(9)$ & $0.0229(10)$ \\
\hline $\mathrm{H} 16 \mathrm{~A}$ & 0.8299 & 1.1954 & 0.8321 & $0.028^{*}$ \\
\hline H16B & 0.9054 & 1.2956 & 0.8393 & $0.028^{*}$ \\
\hline C17A & 0.8234 (3) & $1.4800(7)$ & $0.84240(10)$ & $0.0253(11)$ \\
\hline
\end{tabular}




\begin{tabular}{|c|c|c|c|c|}
\hline $\mathrm{Zr} 2$ & $0.98417(2)$ & 0.64557 (5) & $0.74773(2)$ & $0.01392(9)$ \\
\hline O1B & 0.96739 (17) & 0.4937 (4) & $0.78253(6)$ & $0.0221(7)$ \\
\hline O2B & $1.01413(19)$ & $0.3776(5)$ & $0.82316(7)$ & $0.0298(8)$ \\
\hline O3B & 1.08407 (16) & $0.5300(4)$ & $0.76364(6)$ & $0.0207(7)$ \\
\hline O4B & 1.1952 (2) & $0.4663(6)$ & $0.76049(8)$ & $0.0435(10)$ \\
\hline O5B & 1.00764 (17) & 0.4699 (4) & $0.71588(6)$ & $0.0200(6)$ \\
\hline O6B & $0.98271(18)$ & $0.3468(5)$ & $0.67429(6)$ & $0.0275(8)$ \\
\hline O7B & $0.88833(16)$ & $0.4978(4)$ & $0.73509(6)$ & $0.0208(6)$ \\
\hline O8B & 0.7769 (2) & $0.4490(6)$ & $0.73879(9)$ & 0.0455 (11) \\
\hline N1B & $1.0211(2)$ & $0.8346(6)$ & $0.79070(8)$ & $0.0263(9)$ \\
\hline N2B & $1.0737(2)$ & $0.8271(6)$ & $0.72934(8)$ & $0.0243(8)$ \\
\hline N3B & $0.9286(2)$ & $0.7841(5)$ & $0.70354(7)$ & $0.0235(8)$ \\
\hline N4B & $0.8879(2)$ & $0.8596(6)$ & $0.75476(8)$ & $0.0260(9)$ \\
\hline C1B & $1.0698(3)$ & $1.0021(7)$ & $0.79180(10)$ & $0.0340(12)$ \\
\hline $\mathrm{H} 1 \mathrm{C}$ & 1.0938 & 1.0188 & 0.8109 & $0.041^{*}$ \\
\hline H1D & 1.0413 & 1.1184 & 0.7867 & $0.041^{*}$ \\
\hline C2B & $1.1250(3)$ & $0.9882(8)$ & $0.77382(12)$ & $0.0341(13)$ \\
\hline $\mathrm{H} 2 \mathrm{C}$ & 1.1606 & 1.0891 & 0.7794 & $0.041^{*}$ \\
\hline $\mathrm{H} 2 \mathrm{D}$ & 1.1490 & 0.8632 & 0.7772 & $0.041^{*}$ \\
\hline C3B & $1.0991(3)$ & $1.0069(7)$ & $0.74395(11)$ & $0.0317(11)$ \\
\hline $\mathrm{H} 3 \mathrm{C}$ & 1.0602 & 1.1010 & 0.7412 & $0.038^{*}$ \\
\hline $\mathrm{H} 3 \mathrm{D}$ & 1.1377 & 1.0598 & 0.7354 & $0.038^{*}$ \\
\hline C4B & $1.0474(3)$ & $0.8777(7)$ & $0.69983(9)$ & $0.0291(11)$ \\
\hline $\mathrm{H} 4 \mathrm{C}$ & 1.0768 & 0.9819 & 0.6942 & $0.035^{*}$ \\
\hline H4D & 1.0521 & 0.7649 & 0.6882 & $0.035^{*}$ \\
\hline C5B & $0.9737(3)$ & $0.9388(7)$ & $0.69571(10)$ & $0.0303(11)$ \\
\hline $\mathrm{H} 5 \mathrm{C}$ & 0.9580 & 0.9726 & 0.6763 & $0.036^{*}$ \\
\hline H5D & 0.9691 & 1.0540 & 0.7068 & $0.036^{*}$ \\
\hline C6B & 0.8564 (3) & $0.8633(7)$ & $0.70449(10)$ & $0.0295(11)$ \\
\hline
\end{tabular}




\begin{tabular}{|c|c|c|c|c|}
\hline $\mathrm{H} 6 \mathrm{C}$ & 0.8416 & 0.9473 & 0.6885 & $0.035^{*}$ \\
\hline H6D & 0.8221 & 0.7569 & 0.7036 & $0.035^{*}$ \\
\hline C7B & $0.8566(3)$ & $0.9730(7)$ & 0.72973 (12) & $0.0315(12)$ \\
\hline $\mathrm{H} 7 \mathrm{C}$ & 0.8077 & 1.0097 & 0.7311 & $0.038^{*}$ \\
\hline H7D & 0.8841 & 1.0919 & 0.7290 & $0.038^{*}$ \\
\hline C8B & $0.9057(3)$ & $0.9970(7)$ & $0.77784(10)$ & $0.0322(11)$ \\
\hline $\mathrm{H} 8 \mathrm{C}$ & 0.9282 & 1.1123 & 0.7716 & $0.039^{*}$ \\
\hline H8D & 0.8622 & 1.0372 & 0.7844 & $0.039^{*}$ \\
\hline C9B & $0.9547(3)$ & $0.9048(7)$ & $0.80055(10)$ & $0.0320(12)$ \\
\hline $\mathrm{H} 9 \mathrm{C}$ & 0.9308 & 0.7954 & 0.8077 & $0.038^{*}$ \\
\hline H9D & 0.9677 & 0.9981 & 0.8155 & $0.038^{*}$ \\
\hline C10B & $1.0516(3)$ & $0.6865(7)$ & $0.81084(9)$ & $0.0276(11)$ \\
\hline $\mathrm{H} 10 \mathrm{C}$ & 1.0510 & 0.7337 & 0.8296 & $0.033^{*}$ \\
\hline H10D & 1.1011 & 0.6615 & 0.8090 & $0.033^{*}$ \\
\hline C11B & $1.0094(3)$ & $0.5024(7)$ & $0.80616(9)$ & $0.0205(9)$ \\
\hline C12B & $1.1355(2)$ & $0.6962(7)$ & $0.73046(10)$ & $0.0263(10)$ \\
\hline $\mathrm{H} 12 \mathrm{C}$ & 1.1317 & 0.6266 & 0.7130 & $0.032^{*}$ \\
\hline $\mathrm{H} 12 \mathrm{D}$ & 1.1792 & 0.7734 & 0.7328 & $0.032^{*}$ \\
\hline C13B & $1.1408(3)$ & $0.5543(7)$ & $0.75321(10)$ & $0.0245(10)$ \\
\hline C14B & $0.9220(3)$ & $0.6292(7)$ & $0.68324(10)$ & $0.0245(10)$ \\
\hline $\mathrm{H} 14 \mathrm{C}$ & 0.9286 & 0.6831 & 0.6655 & $0.029^{*}$ \\
\hline H14D & 0.8738 & 0.5756 & 0.6810 & $0.029^{*}$ \\
\hline C15B & $0.9744(3)$ & $0.4704(6)$ & $0.69093(9)$ & $0.0201(9)$ \\
\hline C16B & $0.8336(3)$ & $0.7192(7)$ & $0.76119(10)$ & $0.0270(10)$ \\
\hline $\mathrm{H} 16 \mathrm{C}$ & 0.8449 & 0.6829 & 0.7807 & $0.032^{*}$ \\
\hline $\mathrm{H} 16 \mathrm{D}$ & 0.7867 & 0.7812 & 0.7581 & $0.032^{*}$ \\
\hline C17B & $0.8309(3)$ & $0.5423(7)$ & 0.74395 (10) & $0.0242(10)$ \\
\hline Zr3 & $0.61650(2)$ & $0.93584(5)$ & $0.59569(2)$ & $0.01259(8)$ \\
\hline O1C & $0.54787(16)$ & $1.0550(4)$ & 0.61947 (6) & 0.0167 (6) \\
\hline
\end{tabular}




\begin{tabular}{|c|c|c|c|c|}
\hline $\mathrm{O} 2 \mathrm{C}$ & 0.44296 (18) & 1.1945 (5) & $0.61812(7)$ & $0.0270(7)$ \\
\hline O3C & $0.54762(15)$ & $1.1125(4)$ & $0.56761(6)$ & $0.0169(6)$ \\
\hline O4C & $0.50589(18)$ & $1.2283(5)$ & $0.52644(6)$ & $0.0286(8)$ \\
\hline O5C & $0.68470(15)$ & $1.1367(4)$ & $0.58198(6)$ & $0.0179(6)$ \\
\hline O6C & 0.78919 (17) & $1.2398(5)$ & $0.57495(7)$ & $0.0288(8)$ \\
\hline O7C & $0.68136(16)$ & $1.0100(4)$ & $0.63314(6)$ & $0.0184(6)$ \\
\hline O8C & $0.6949(2)$ & $0.9930(5)$ & $0.67855(7)$ & $0.0348(9)$ \\
\hline N1C & $0.50009(19)$ & $0.7586(5)$ & $0.59010(7)$ & $0.0157(7)$ \\
\hline $\mathrm{N} 2 \mathrm{C}$ & $0.62043(19)$ & $0.8311(5)$ & $0.54863(7)$ & $0.0168(7)$ \\
\hline N3C & $0.73157(19)$ & $0.7827(5)$ & $0.59534(7)$ & $0.0161(7)$ \\
\hline $\mathrm{N} 4 \mathrm{C}$ & $0.62806(18)$ & $0.6534(5)$ & $0.62530(7)$ & $0.0160(7)$ \\
\hline C1C & $0.4736(2)$ & $0.6320(6)$ & $0.56630(9)$ & $0.0205(9)$ \\
\hline H1E & 0.4215 & 0.6293 & 0.5637 & $0.025^{*}$ \\
\hline $\mathrm{H} 1 \mathrm{~F}$ & 0.4906 & 0.5002 & 0.5708 & $0.025^{\star}$ \\
\hline $\mathrm{C} 2 \mathrm{C}$ & $0.4955(2)$ & $0.6900(6)$ & $0.53974(9)$ & $0.0202(9)$ \\
\hline $\mathrm{H} 2 \mathrm{E}$ & 0.4833 & 0.8264 & 0.5362 & $0.024^{*}$ \\
\hline $\mathrm{H} 2 \mathrm{~F}$ & 0.4681 & 0.6140 & 0.5248 & $0.024^{*}$ \\
\hline C3C & $0.5738(2)$ & $0.6626(6)$ & $0.53914(9)$ & $0.0209(9)$ \\
\hline H3E & 0.5906 & 0.5510 & 0.5507 & $0.025^{\star}$ \\
\hline H3F & 0.5795 & 0.6313 & 0.5202 & $0.025^{*}$ \\
\hline $\mathrm{C} 4 \mathrm{C}$ & $0.6939(2)$ & $0.7806(6)$ & $0.54540(9)$ & $0.0197(9)$ \\
\hline $\mathrm{H} 4 \mathrm{E}$ & 0.7207 & 0.8990 & 0.5434 & $0.024^{*}$ \\
\hline $\mathrm{H} 4 \mathrm{~F}$ & 0.6928 & 0.7030 & 0.5287 & $0.024^{*}$ \\
\hline C5C & $0.7298(2)$ & $0.6699(6)$ & $0.56963(9)$ & $0.0206(9)$ \\
\hline H5E & 0.7044 & 0.5481 & 0.5710 & $0.025^{*}$ \\
\hline $\mathrm{H} 5 \mathrm{~F}$ & 0.7785 & 0.6389 & 0.5672 & $0.025^{\star}$ \\
\hline $\mathrm{C} 6 \mathrm{C}$ & $0.7524(3)$ & $0.6525(7)$ & $0.61911(9)$ & $0.0217(10)$ \\
\hline H6E & 0.7682 & 0.7286 & 0.6358 & $0.026^{*}$ \\
\hline $\mathrm{H} 6 \mathrm{~F}$ & 0.7919 & 0.5695 & 0.6159 & $0.026^{*}$ \\
\hline
\end{tabular}




\begin{tabular}{|c|c|c|c|c|}
\hline C7C & $0.6900(3)$ & $0.5321(6)$ & $0.62260(10)$ & $0.0219(10)$ \\
\hline H7E & 0.6779 & 0.4464 & 0.6067 & $0.026^{*}$ \\
\hline $\mathrm{H} 7 \mathrm{~F}$ & 0.7022 & 0.4513 & 0.6390 & $0.026^{*}$ \\
\hline $\mathrm{C} 8 \mathrm{C}$ & $0.5651(3)$ & $0.5270(6)$ & $0.62123(10)$ & $0.0204(9)$ \\
\hline H8E & 0.5645 & 0.4493 & 0.6378 & $0.024^{*}$ \\
\hline $\mathrm{H} 8 \mathrm{~F}$ & 0.5673 & 0.4386 & 0.6058 & $0.024^{*}$ \\
\hline C9C & $0.4995(2)$ & $0.6441(6)$ & $0.61536(9)$ & $0.0182(9)$ \\
\hline H9E & 0.4578 & 0.5592 & 0.6129 & $0.022^{*}$ \\
\hline $\mathrm{H} 9 \mathrm{~F}$ & 0.4967 & 0.7308 & 0.6309 & $0.022^{*}$ \\
\hline C10C & $0.4482(2)$ & $0.9165(7)$ & $0.59114(9)$ & $0.0198(9)$ \\
\hline $\mathrm{H} 10 \mathrm{E}$ & 0.4052 & 0.8642 & 0.5968 & $0.024^{*}$ \\
\hline $\mathrm{H} 10 \mathrm{~F}$ & 0.4349 & 0.9731 & 0.5728 & $0.024^{*}$ \\
\hline C11C & $0.4794(2)$ & $1.0698(6)$ & $0.61101(9)$ & $0.0177(9)$ \\
\hline C12C & $0.5976(3)$ & $0.9977(7)$ & $0.53042(9)$ & $0.0204(10)$ \\
\hline $\mathrm{H} 12 \mathrm{E}$ & 0.5759 & 0.9502 & 0.5122 & $0.024^{*}$ \\
\hline $\mathrm{H} 12 \mathrm{~F}$ & 0.6394 & 1.0749 & 0.5282 & $0.024^{*}$ \\
\hline C13C & $0.5455(2)$ & $1.1226(6)$ & $0.54166(9)$ & $0.0199(9)$ \\
\hline C14C & $0.7844(2)$ & $0.9378(6)$ & $0.59640(10)$ & $0.0193(9)$ \\
\hline H14E & 0.8230 & 0.8951 & 0.5870 & $0.023^{*}$ \\
\hline $\mathrm{H} 14 \mathrm{~F}$ & 0.8048 & 0.9644 & 0.6156 & $0.023^{*}$ \\
\hline C15C & $0.7525(2)$ & $1.1189(6)$ & $0.58320(9)$ & $0.0194(9)$ \\
\hline C16C & $0.6378(3)$ & $0.7361(7)$ & $0.65354(9)$ & $0.0203(9)$ \\
\hline H16E & 0.6655 & 0.6452 & 0.6664 & $0.024^{*}$ \\
\hline $\mathrm{H} 16 \mathrm{~F}$ & 0.5910 & 0.7508 & 0.6591 & $0.024^{*}$ \\
\hline C17C & $0.6746(3)$ & $0.9275(7)$ & $0.65582(10)$ & $0.0210(10)$ \\
\hline 01 & $0.7400(3)$ & $0.9006(6)$ & $0.81582(8)$ & $0.0454(10)$ \\
\hline $\mathrm{H} 11$ & $0.764(2)$ & $0.803(5)$ & $0.8175(9)$ & $0.024(14)^{*}$ \\
\hline $\mathrm{H} 12$ & $0.720(3)$ & $0.897(8)$ & $0.8000(6)$ & $0.06(2)^{*}$ \\
\hline $\mathrm{O} 2$ & $0.6500(3)$ & $0.7903(8)$ & $0.85387(10)$ & $0.0560(12)$ \\
\hline
\end{tabular}




\begin{tabular}{|c|c|c|c|c|}
\hline $\mathrm{H} 21$ & 0.676 (2) & $0.834(9)$ & $0.8438(11)$ & $0.06(2)^{*}$ \\
\hline $\mathrm{H} 22$ & $0.6105(13)$ & $0.825(10)$ & $0.8464(11)$ & $0.06(2)^{*}$ \\
\hline O3 & $0.6848(2)$ & $0.9531(6)$ & $0.97189(8)$ & $0.0348(9)$ \\
\hline H31 & $0.663(4)$ & $1.054(6)$ & $0.9696(11)$ & $0.07(3)^{*}$ \\
\hline H32 & $0.690(3)$ & $0.912(6)$ & $0.9571(5)$ & $0.022(13)^{*}$ \\
\hline O4 & $0.6282(2)$ & $1.3038(6)$ & $0.96380(9)$ & $0.0393(9)$ \\
\hline $\mathrm{H} 41$ & $0.641(3)$ & $1.408(6)$ & $0.9704(17)$ & $0.10(3)^{*}$ \\
\hline $\mathrm{H} 42$ & $0.5855(9)$ & 1.304 (8) & $0.9636(12)$ & $0.039(18)^{*}$ \\
\hline O5 & 0.6766 (2) & $0.6024(6)$ & $0.99721(10)$ & $0.0462(11)$ \\
\hline H51 & $0.674(2)$ & $0.705(4)$ & $0.9893(8)$ & $0.016(12)^{\star}$ \\
\hline H52 & $0.7162(17)$ & $0.597(9)$ & $1.0061(13)$ & $0.07(3)^{*}$ \\
\hline 06 & $0.7598(2)$ & $1.3433(6)$ & $0.68332(8)$ & $0.0446(10)$ \\
\hline H61 & $0.743(3)$ & $1.236(4)$ & $0.6829(11)$ & $0.05(2)^{*}$ \\
\hline H62 & $0.760(4)$ & $1.383(8)$ & $0.6987(6)$ & $0.06(2)^{\star}$ \\
\hline 07 & $0.8479(3)$ & $0.2243(8)$ & $0.64502(10)$ & $0.0533(12)$ \\
\hline $\mathrm{H} 71$ & $0.820(2)$ & $0.235(12)$ & $0.6557(11)$ & $0.08(3)^{\star}$ \\
\hline $\mathrm{H} 72$ & $0.8863(15)$ & $0.258(9)$ & $0.6535(10)$ & $0.049(19)^{*}$ \\
\hline O8 & $0.8191(3)$ & $1.0465(6)$ & $0.50202(10)$ & $0.0473(11)$ \\
\hline $\mathrm{H} 81$ & $0.825(3)$ & $1.149(5)$ & $0.5097(12)$ & $0.06(2)^{*}$ \\
\hline H82 & $0.7775(12)$ & $1.038(8)$ & $0.4955(13)$ & $0.05(2)^{*}$ \\
\hline O9 & $0.8689(2)$ & $0.7518(6)$ & $0.53532(9)$ & $0.0382(9)$ \\
\hline H91 & $0.850(2)$ & $0.832(6)$ & $0.5242(8)$ & $0.027(15)^{*}$ \\
\hline H92 & $0.9090(14)$ & $0.747(9)$ & $0.5317(12)$ & $0.06(2)^{\star}$ \\
\hline 010 & $0.8118(2)$ & $1.3998(5)$ & $0.52682(7)$ & $0.0332(8)$ \\
\hline $\mathrm{H} 101$ & $0.806(3)$ & $1.365(6)$ & $0.5419(5)$ & $0.025(14)^{*}$ \\
\hline $\mathrm{H} 102$ & $0.821(4)$ & $1.514(3)$ & $0.5279(11)$ & $0.06(2)^{*}$ \\
\hline
\end{tabular}


Table S3. Atomic displacement parameters $\left(\AA^{2}\right)$

\begin{tabular}{|c|c|c|c|c|c|c|}
\hline & $U^{11}$ & $U^{22}$ & $U^{\beta 3}$ & $u^{12}$ & $U^{13}$ & $u^{33}$ \\
\hline Zr1 & $0.01429(19)$ & 0.01012 (17) & $0.01510(17)$ & $0.00042(16)$ & $0.00320(13)$ & $0.00088(14)$ \\
\hline O1A & $0.0184(16)$ & $0.0182(15)$ & $0.0195(15)$ & $0.0010(13)$ & $0.0056(12)$ & $0.0039(11)$ \\
\hline $\mathrm{O} 2 \mathrm{~A}$ & $0.0273(19)$ & $0.0212(17)$ & $0.041(2)$ & $-0.0034(14)$ & $0.0142(15)$ & $0.0066(14)$ \\
\hline O3A & $0.0186(16)$ & $0.0187(15)$ & $0.0209(15)$ & $-0.0042(12)$ & $0.0043(12)$ & $-0.0034(11)$ \\
\hline O4A & $0.0305(19)$ & $0.0296(18)$ & $0.0268(17)$ & $-0.0100(15)$ & $0.0017(14)$ & $-0.0098(14)$ \\
\hline O5A & $0.0201(16)$ & $0.0140(15)$ & $0.0256(16)$ & $-0.0004(12)$ & $0.0074(13)$ & $0.0004(11)$ \\
\hline O6A & $0.0232(18)$ & $0.0229(17)$ & $0.044(2)$ & $0.0053(14)$ & $0.0084(15)$ & $-0.0059(15)$ \\
\hline O7A & $0.0219(17)$ & $0.0189(16)$ & $0.0180(15)$ & $0.0038(13)$ & $0.0017(12)$ & $0.0029(11)$ \\
\hline O8A & $0.076(3)$ & $0.048(3)$ & $0.022(2)$ & $0.032(2)$ & $0.006(2)$ & $0.0100(16)$ \\
\hline $\mathrm{N} 1 \mathrm{~A}$ & $0.0172(19)$ & 0.0159 (17) & $0.0170(17)$ & $0.0038(15)$ & $0.0032(14)$ & $0.0009(13)$ \\
\hline $\mathrm{N} 2 \mathrm{~A}$ & $0.0190(19)$ & $0.0139(17)$ & $0.0162(17)$ & $-0.0009(14)$ & $0.0029(14)$ & $0.0002(13)$ \\
\hline N3A & $0.0177(19)$ & $0.0131(17)$ & $0.0196(18)$ & $-0.0029(14)$ & $0.0026(14)$ & $-0.0021(13)$ \\
\hline N4A & $0.0192(19)$ & $0.0139(16)$ & $0.0176(17)$ & $0.0006(14)$ & $0.0032(14)$ & $0.0007(13)$ \\
\hline C1A & $0.022(2)$ & $0.017(2)$ & 0.026 (2) & $0.0047(18)$ & $0.0008(18)$ & $0.0058(17)$ \\
\hline $\mathrm{C} 2 \mathrm{~A}$ & $0.025(2)$ & $0.020(2)$ & 0.018 (2) & $0.0041(18)$ & $0.0014(17)$ & $0.0052(16)$ \\
\hline C3A & $0.026(3)$ & $0.016(2)$ & 0.026 (2) & $0.0032(19)$ & $0.0037(19)$ & $0.0064(17)$ \\
\hline C4A & $0.020(2)$ & $0.022(2)$ & $0.021(2)$ & $-0.0014(18)$ & $0.0077(17)$ & $0.0021(16)$ \\
\hline C5A & $0.019(2)$ & $0.020(2)$ & $0.028(2)$ & $-0.0045(18)$ & $0.0080(18)$ & $0.0032(17)$ \\
\hline $\mathrm{C} 6 \mathrm{~A}$ & $0.018(2)$ & $0.021(2)$ & 0.027 (2) & $-0.0059(19)$ & $0.0025(18)$ & $-0.0034(18)$ \\
\hline C7A & $0.028(3)$ & $0.014(2)$ & $0.028(2)$ & $-0.0034(18)$ & $0.0045(19)$ & $-0.0065(17)$ \\
\hline C8A & $0.032(3)$ & $0.0102(19)$ & 0.024 (2) & $0.0071(18)$ & $0.0064(19)$ & $-0.0013(16)$ \\
\hline C9A & $0.025(2)$ & $0.017(2)$ & $0.020(2)$ & $0.0084(18)$ & $0.0070(18)$ & $-0.0007(16)$ \\
\hline C10A & $0.014(2)$ & $0.020(2)$ & 0.028 (3) & $0.0024(18)$ & $0.0050(18)$ & $0.0034(17)$ \\
\hline C11A & $0.021(2)$ & $0.018(2)$ & $0.023(2)$ & $0.0012(18)$ & $0.0077(17)$ & $-0.0022(16)$ \\
\hline C12A & $0.022(3)$ & $0.021(2)$ & $0.019(2)$ & $-0.0022(19)$ & $0.0024(18)$ & $-0.0051(16)$ \\
\hline C13A & $0.020(2)$ & $0.015(2)$ & $0.019(2)$ & $0.0024(17)$ & $0.0028(16)$ & $-0.0021(15)$ \\
\hline C14A & 0.016 (2) & 0.018 (2) & 0.029 (3) & $0.0010(18)$ & $0.0033(19)$ & $0.0004(17)$ \\
\hline
\end{tabular}




\begin{tabular}{|c|c|c|c|c|c|c|}
\hline C15A & $0.020(2)$ & 0.018 (2) & 0.019 (2) & $0.0019(18)$ & $0.0027(17)$ & $0.0045(16)$ \\
\hline C16A & 0.025 (3) & 0.026 (2) & 0.018 (2) & $0.005(2)$ & $0.0038(18)$ & $-0.0001(18)$ \\
\hline C17A & $0.025(3)$ & $0.029(3)$ & $0.021(2)$ & $0.006(2)$ & $0.002(2)$ & $0.0043(18)$ \\
\hline Zr2 & $0.0160(2)$ & $0.01155(18)$ & $0.01501(16)$ & $0.00001(16)$ & $0.00493(15)$ & $0.00014(14)$ \\
\hline O1B & $0.0272(18)$ & $0.0213(16)$ & $0.0184(15)$ & $-0.0036(13)$ & $0.0052(13)$ & $0.0018(12)$ \\
\hline $\mathrm{O} 2 \mathrm{~B}$ & $0.035(2)$ & $0.0315(19)$ & $0.0236(18)$ & $0.0058(15)$ & $0.0056(15)$ & $0.0091(14)$ \\
\hline O3B & $0.0205(17)$ & $0.0223(16)$ & $0.0186(15)$ & $0.0022(13)$ & $0.0012(12)$ & $0.0021(11)$ \\
\hline O4B & $0.024(2)$ & $0.066(3)$ & $0.042(2)$ & $0.0227(19)$ & $0.0099(17)$ & $0.0174(19)$ \\
\hline O5B & $0.0247(17)$ & $0.0168(15)$ & $0.0178(15)$ & $0.0022(13)$ & $0.0020(12)$ & $-0.0018(11)$ \\
\hline O6B & $0.036(2)$ & $0.0272(18)$ & $0.0201(16)$ & $-0.0007(15)$ & $0.0070(14)$ & $-0.0042(13)$ \\
\hline O7B & $0.0190(17)$ & $0.0202(16)$ & $0.0228(16)$ & $-0.0024(13)$ & $0.0028(13)$ & $-0.0024(12)$ \\
\hline O8B & $0.028(2)$ & 0.057 (3) & $0.054(3)$ & $-0.0225(19)$ & $0.0146(18)$ & $-0.021(2)$ \\
\hline N1B & $0.035(2)$ & $0.022(2)$ & $0.0226(19)$ & $-0.0064(17)$ & $0.0075(17)$ & $-0.0053(15)$ \\
\hline N2B & $0.021(2)$ & $0.024(2)$ & $0.030(2)$ & $0.0006(17)$ & $0.0091(16)$ & $0.0043(16)$ \\
\hline N3B & $0.031(2)$ & $0.0205(19)$ & $0.0193(18)$ & $0.0073(17)$ & $0.0052(16)$ & $0.0034(14)$ \\
\hline N4B & $0.025(2)$ & $0.023(2)$ & $0.031(2)$ & $0.0005(17)$ & $0.0082(17)$ & $-0.0064(16)$ \\
\hline C1B & 0.038 (3) & 0.031 (3) & 0.033 (3) & $-0.012(2)$ & $0.005(2)$ & $-0.011(2)$ \\
\hline C2B & $0.032(3)$ & $0.028(3)$ & $0.044(3)$ & $-0.011(2)$ & $0.011(2)$ & $-0.011(2)$ \\
\hline C3B & $0.026(3)$ & $0.029(3)$ & $0.041(3)$ & $-0.006(2)$ & $0.006(2)$ & $0.004(2)$ \\
\hline C4B & $0.039(3)$ & $0.028(3)$ & $0.023(2)$ & $-0.004(2)$ & $0.013(2)$ & $0.0086(18)$ \\
\hline C5B & 0.038 (3) & 0.029 (3) & 0.024 (2) & $-0.001(2)$ & $0.004(2)$ & $0.0089(19)$ \\
\hline C6B & $0.027(3)$ & $0.032(3)$ & $0.028(2)$ & $0.009(2)$ & $0.0004(19)$ & $0.005(2)$ \\
\hline C7B & $0.027(3)$ & $0.023(3)$ & $0.047(3)$ & $0.012(2)$ & $0.012(2)$ & $0.008(2)$ \\
\hline C8B & $0.036(3)$ & 0.027 (3) & 0.034 (3) & $0.006(2)$ & $0.009(2)$ & $-0.006(2)$ \\
\hline C9B & 0.049 (3) & 0.024 (3) & $0.025(2)$ & $0.006(2)$ & $0.014(2)$ & $-0.0063(19)$ \\
\hline C10B & $0.030(3)$ & 0.037 (3) & $0.015(2)$ & $-0.006(2)$ & $0.0018(19)$ & $-0.0058(19)$ \\
\hline C11B & $0.022(2)$ & $0.023(2)$ & $0.018(2)$ & $0.0060(19)$ & $0.0082(17)$ & $0.0008(17)$ \\
\hline C12B & $0.016(2)$ & 0.035 (3) & $0.030(2)$ & $0.001(2)$ & $0.0092(18)$ & $0.001(2)$ \\
\hline C13B & $0.020(2)$ & 0.027 (3) & 0.027 (3) & 0.004 (2) & 0.0050 (19) & $-0.0016(18)$ \\
\hline
\end{tabular}




\begin{tabular}{|c|c|c|c|c|c|c|}
\hline C14B & $0.026(3)$ & 0.029 (3) & 0.017 (2) & $0.002(2)$ & $0.0001(18)$ & $0.0006(17)$ \\
\hline C15B & $0.022(2)$ & 0.021 (2) & 0.019 (2) & $-0.0043(19)$ & $0.0053(17)$ & $0.0004(16)$ \\
\hline C16B & $0.020(2)$ & $0.031(3)$ & $0.033(3)$ & $0.001(2)$ & $0.011(2)$ & $-0.004(2)$ \\
\hline C17B & $0.021(3)$ & $0.031(3)$ & $0.022(2)$ & $-0.002(2)$ & $0.0067(18)$ & $0.0016(18)$ \\
\hline Zr3 & $0.01361(19)$ & $0.00982(17)$ & $0.01459(17)$ & $-0.00049(15)$ & $0.00310(13)$ & $-0.00080(14)$ \\
\hline O1C & $0.0178(16)$ & $0.0135(14)$ & 0.0201 (15) & $-0.0019(12)$ & 0.0064 (12) & $-0.0034(11)$ \\
\hline $\mathrm{O} 2 \mathrm{C}$ & $0.0244(18)$ & $0.0219(17)$ & $0.0366(19)$ & $0.0032(14)$ & $0.0102(14)$ & $-0.0043(14)$ \\
\hline $\mathrm{O} 3 \mathrm{C}$ & $0.0174(15)$ & $0.0164(15)$ & $0.0173(14)$ & $0.0037(12)$ & $0.0047(11)$ & $0.0023(11)$ \\
\hline $\mathrm{O} 4 \mathrm{C}$ & 0.034 (2) & $0.0274(18)$ & 0.0229 (17) & $0.0105(15)$ & $0.0014(14)$ & $0.0090(13)$ \\
\hline O5C & $0.0160(15)$ & $0.0143(15)$ & $0.0239(16)$ & $-0.0011(12)$ & $0.0049(12)$ & $0.0008(11)$ \\
\hline $\mathrm{O} 6 \mathrm{C}$ & $0.0209(18)$ & $0.0246(18)$ & $0.042(2)$ & $-0.0061(14)$ & $0.0087(15)$ & $0.0075(14)$ \\
\hline O7C & $0.0178(16)$ & $0.0170(15)$ & $0.0200(16)$ & $-0.0024(12)$ & $0.0023(12)$ & $-0.0026(11)$ \\
\hline $\mathrm{O} 8 \mathrm{C}$ & $0.042(2)$ & 0.039 (2) & 0.0212 (18) & $-0.0130(18)$ & $0.0016(16)$ & $-0.0080(14)$ \\
\hline N1C & $0.0179(18)$ & $0.0118(16)$ & $0.0180(17)$ & $-0.0015(14)$ & $0.0046(13)$ & $-0.0017(12)$ \\
\hline $\mathrm{N} 2 \mathrm{C}$ & $0.0171(18)$ & $0.0167(18)$ & $0.0168(17)$ & $-0.0004(15)$ & $0.0038(14)$ & $-0.0004(13)$ \\
\hline N3C & $0.0165(18)$ & $0.0133(17)$ & $0.0186(17)$ & $0.0014(14)$ & $0.0027(14)$ & $0.0013(13)$ \\
\hline $\mathrm{N} 4 \mathrm{C}$ & $0.0188(18)$ & $0.0138(16)$ & $0.0154(17)$ & $-0.0018(14)$ & $0.0032(13)$ & $-0.0023(12)$ \\
\hline $\mathrm{C} 1 \mathrm{C}$ & $0.021(2)$ & $0.019(2)$ & $0.021(2)$ & $-0.0037(18)$ & $-0.0009(17)$ & $-0.0037(16)$ \\
\hline $\mathrm{C} 2 \mathrm{C}$ & $0.020(2)$ & $0.018(2)$ & $0.021(2)$ & $-0.0039(18)$ & $-0.0003(17)$ & $-0.0050(16)$ \\
\hline $\mathrm{C} 3 \mathrm{C}$ & $0.026(2)$ & $0.019(2)$ & 0.019 (2) & $-0.0039(19)$ & $0.0058(17)$ & $-0.0069(16)$ \\
\hline $\mathrm{C} 4 \mathrm{C}$ & $0.018(2)$ & $0.021(2)$ & $0.021(2)$ & $0.0043(18)$ & $0.0058(17)$ & $-0.0033(16)$ \\
\hline $\mathrm{C} 5 \mathrm{C}$ & $0.024(2)$ & $0.018(2)$ & $0.020(2)$ & $0.0035(18)$ & $0.0045(17)$ & $-0.0058(16)$ \\
\hline C6C & $0.022(2)$ & $0.023(2)$ & $0.021(2)$ & $0.0076(19)$ & $0.0030(18)$ & $0.0050(17)$ \\
\hline C7C & $0.027(3)$ & $0.014(2)$ & $0.025(2)$ & $0.0066(18)$ & $0.0056(19)$ & $0.0055(16)$ \\
\hline $\mathrm{C} 8 \mathrm{C}$ & $0.026(3)$ & $0.014(2)$ & $0.022(2)$ & $-0.0047(18)$ & $0.0062(19)$ & $0.0007(15)$ \\
\hline C9C & $0.021(2)$ & $0.015(2)$ & 0.019 (2) & $-0.0042(18)$ & $0.0052(17)$ & $-0.0032(15)$ \\
\hline C10C & $0.017(2)$ & $0.020(2)$ & $0.023(2)$ & $-0.0007(18)$ & $0.0037(18)$ & $-0.0027(17)$ \\
\hline C11C & $0.018(2)$ & $0.016(2)$ & $0.020(2)$ & $-0.0003(17)$ & $0.0074(17)$ & $0.0030(16)$ \\
\hline C12C & 0.028 (3) & 0.019 (2) & 0.015 (2) & $0.0026(19)$ & 0.0078 (18) & $0.0042(16)$ \\
\hline
\end{tabular}




\begin{tabular}{|l|l|l|l|l|l|l|}
\hline C13C & $0.017(2)$ & $0.015(2)$ & $0.027(2)$ & $-0.0019(17)$ & $0.0011(17)$ & $0.0018(16)$ \\
\hline C14C & $0.014(2)$ & $0.020(2)$ & $0.025(2)$ & $-0.0020(17)$ & $0.0055(18)$ & $-0.0003(17)$ \\
\hline C15C & $0.019(2)$ & $0.018(2)$ & $0.022(2)$ & $-0.0033(18)$ & $0.0051(17)$ & $-0.0038(16)$ \\
\hline C16C & $0.023(2)$ & $0.023(2)$ & $0.015(2)$ & $0.0005(18)$ & $0.0025(17)$ & $0.0020(15)$ \\
\hline C17C & $0.020(3)$ & $0.024(2)$ & $0.018(2)$ & $0.0034(19)$ & $0.0034(18)$ & $-0.0015(17)$ \\
\hline O1 & $0.060(3)$ & $0.040(2)$ & $0.032(2)$ & $0.012(2)$ & $-0.003(2)$ & $-0.0060(17)$ \\
\hline O2 & $0.037(3)$ & $0.079(3)$ & $0.050(3)$ & $0.007(3)$ & $0.003(2)$ & $0.023(2)$ \\
\hline O3 & $0.047(2)$ & $0.029(2)$ & $0.028(2)$ & $0.0050(19)$ & $0.0079(17)$ & $0.0040(15)$ \\
\hline O4 & $0.036(2)$ & $0.032(2)$ & $0.052(3)$ & $0.0051(18)$ & $0.0124(19)$ & $-0.0029(18)$ \\
\hline O5 & $0.047(3)$ & $0.032(2)$ & $0.056(3)$ & $-0.013(2)$ & $-0.004(2)$ & $0.012(2)$ \\
\hline O6 & $0.060(3)$ & $0.040(2)$ & $0.034(2)$ & $-0.022(2)$ & $0.008(2)$ & $-0.0014(18)$ \\
\hline O7 & $0.037(3)$ & $0.070(3)$ & $0.050(3)$ & $-0.008(2)$ & $-0.001(2)$ & $-0.024(2)$ \\
\hline O8 & $0.045(3)$ & $0.034(2)$ & $0.059(3)$ & $0.012(2)$ & $-0.004(2)$ & $-0.013(2)$ \\
\hline O9 & $0.032(2)$ & $0.034(2)$ & $0.050(3)$ & $-0.0019(18)$ & $0.0085(19)$ & $0.0064(18)$ \\
\hline O10 & $0.044(2)$ & $0.026(2)$ & $0.030(2)$ & $-0.0060(18)$ & $0.0075(17)$ & $-0.0025(15)$ \\
\hline
\end{tabular}

Table S4. Geometric parameters ( $\AA$, $\left.{ }^{\circ}\right)$

\begin{tabular}{|l|l|l|l|}
\hline Zr1-O1A & $2.099(3)$ & C6B-H6C & 0.9900 \\
\hline Zr1-O5A & $2.114(3)$ & C6B-H6D & 0.9900 \\
\hline Zr1-O7A & $2.129(3)$ & C7B-H7C & 0.9900 \\
\hline Zr1-O3A & $2.160(3)$ & C7B-H7D & 0.9900 \\
\hline Zr1-N4A & $2.451(4)$ & C8B-C9B & $1.491(7)$ \\
\hline Zr1—N2A & $2.466(3)$ & C8B-H8C & 0.9900 \\
\hline Zr1-N3A & $2.468(4)$ & C8B-H8D & 0.9900 \\
\hline Zr1-N1A & $2.538(4)$ & C9B-H9C & 0.9900 \\
\hline O1A-C11A & $1.305(6)$ & C9B-H9D & 0.9900 \\
\hline O2A-C11A & $1.218(5)$ & C10B-C11B & $1.519(7)$ \\
\hline O3A-C13A & $1.287(5)$ & C10B-H10C & 0.9900 \\
\hline
\end{tabular}




\begin{tabular}{|c|c|c|c|}
\hline$O 4 A-C 13 A$ & $1.226(5)$ & C10B-H10D & 0.9900 \\
\hline O5A-C15A & $1.307(5)$ & C12B-C13B & $1.496(7)$ \\
\hline O6A-C15A & $1.223(5)$ & $\mathrm{C} 12 \mathrm{~B}-\mathrm{H} 12 \mathrm{C}$ & 0.9900 \\
\hline O7A-C17A & $1.282(6)$ & C12B-H12D & 0.9900 \\
\hline$O 8 A-C 17 A$ & $1.223(6)$ & C14B-C15B & $1.506(7)$ \\
\hline $\mathrm{N} 1 \mathrm{~A}-\mathrm{C} 1 \mathrm{~A}$ & $1.496(5)$ & C14B-H14C & 0.9900 \\
\hline $\mathrm{N} 1 \mathrm{~A}-\mathrm{C} 10 \mathrm{~A}$ & $1.496(6)$ & C14B-H14D & 0.9900 \\
\hline N1A-C9A & $1.501(5)$ & C16B-C17B & $1.501(7)$ \\
\hline $\mathrm{N} 2 \mathrm{~A}-\mathrm{C} 12 \mathrm{~A}$ & $1.488(5)$ & C16B-H16C & 0.9900 \\
\hline $\mathrm{N} 2 \mathrm{~A}-\mathrm{C} 4 \mathrm{~A}$ & $1.496(6)$ & C16B-H16D & 0.9900 \\
\hline $\mathrm{N} 2 \mathrm{~A}-\mathrm{C} 3 \mathrm{~A}$ & $1.514(5)$ & $\mathrm{Zr3}-\mathrm{O} 1 \mathrm{C}$ & 2.091 (3) \\
\hline N3A-C14A & $1.484(6)$ & Zr3-O5C & $2.113(3)$ \\
\hline $\mathrm{N} 3 \mathrm{~A}-\mathrm{C} 6 \mathrm{~A}$ & $1.493(6)$ & Zr3-O7C & $2.126(3)$ \\
\hline$N 3 A-C 5 A$ & $1.494(5)$ & $\mathrm{Zr} 3-\mathrm{O} 3 \mathrm{C}$ & $2.148(3)$ \\
\hline N4A-C16A & $1.485(5)$ & $\mathrm{Zr3}-\mathrm{N} 4 \mathrm{C}$ & $2.451(3)$ \\
\hline $\mathrm{N} 4 \mathrm{~A}-\mathrm{C} 7 \mathrm{~A}$ & $1.494(6)$ & Zr3-N3C & $2.465(4)$ \\
\hline $\mathrm{N} 4 \mathrm{~A}-\mathrm{C} 8 \mathrm{~A}$ & $1.496(6)$ & $\mathrm{Zr3}-\mathrm{N} 2 \mathrm{C}$ & $2.468(3)$ \\
\hline $\mathrm{C} 1 \mathrm{~A}-\mathrm{C} 2 \mathrm{~A}$ & $1.516(6)$ & Zr3-N1C & $2.536(4)$ \\
\hline $\mathrm{C} 1 \mathrm{~A}-\mathrm{H} 1 \mathrm{~A}$ & 0.9900 & O1C-C11C & $1.319(5)$ \\
\hline $\mathrm{C} 1 \mathrm{~A}-\mathrm{H} 1 \mathrm{~B}$ & 0.9900 & $\mathrm{O} 2 \mathrm{C}-\mathrm{C} 11 \mathrm{C}$ & $1.209(5)$ \\
\hline $\mathrm{C} 2 \mathrm{~A}-\mathrm{C} 3 \mathrm{~A}$ & $1.510(7)$ & $\mathrm{O} 3 \mathrm{C}-\mathrm{C} 13 \mathrm{C}$ & $1.288(5)$ \\
\hline $\mathrm{C} 2 \mathrm{~A}-\mathrm{H} 2 \mathrm{~A}$ & 0.9900 & O4C-C13C & $1.228(5)$ \\
\hline $\mathrm{C} 2 \mathrm{~A}-\mathrm{H} 2 \mathrm{~B}$ & 0.9900 & O5C-C15C & $1.303(5)$ \\
\hline $\mathrm{C} 3 \mathrm{~A}-\mathrm{H} 3 \mathrm{~A}$ & 0.9900 & O6C-C15C & $1.216(5)$ \\
\hline СЗА-HЗB & 0.9900 & O7C-C17C & $1.294(6)$ \\
\hline C4A-C5A & $1.498(6)$ & $\mathrm{O} 8 \mathrm{C}-\mathrm{C} 17 \mathrm{C}$ & $1.222(6)$ \\
\hline $\mathrm{C} 4 \mathrm{~A}-\mathrm{H} 4 \mathrm{~A}$ & 0.9900 & $\mathrm{~N} 1 \mathrm{C}-\mathrm{C} 9 \mathrm{C}$ & $1.492(5)$ \\
\hline $\mathrm{C} 4 \mathrm{~A}-\mathrm{H} 4 \mathrm{~B}$ & 0.9900 & $\mathrm{~N} 1 \mathrm{C}-\mathrm{C} 10 \mathrm{C}$ & $1.495(6)$ \\
\hline C5A-H5A & 0.9900 & $\mathrm{~N} 1 \mathrm{C}-\mathrm{C} 1 \mathrm{C}$ & $1.498(5)$ \\
\hline
\end{tabular}




\begin{tabular}{|c|c|c|c|}
\hline $\mathrm{C} 5 \mathrm{~A}-\mathrm{H} 5 \mathrm{~B}$ & 0.9900 & $\mathrm{~N} 2 \mathrm{C}-\mathrm{C} 4 \mathrm{C}$ & $1.493(5)$ \\
\hline C6A-C7A & $1.491(7)$ & $\mathrm{N} 2 \mathrm{C}-\mathrm{C} 12 \mathrm{C}$ & $1.496(5)$ \\
\hline $\mathrm{C} 6 \mathrm{~A}-\mathrm{H} 6 \mathrm{~A}$ & 0.9900 & $\mathrm{~N} 2 \mathrm{C}-\mathrm{C} 3 \mathrm{C}$ & $1.508(5)$ \\
\hline $\mathrm{C} 6 \mathrm{~A}-\mathrm{H} 6 \mathrm{~B}$ & 0.9900 & $\mathrm{~N} 3 \mathrm{C}-\mathrm{C} 14 \mathrm{C}$ & $1.482(6)$ \\
\hline C7A-H7A & 0.9900 & $\mathrm{~N} 3 \mathrm{C}-\mathrm{C} 6 \mathrm{C}$ & $1.493(5)$ \\
\hline C7A-H7B & 0.9900 & $\mathrm{~N} 3 \mathrm{C}-\mathrm{C} 5 \mathrm{C}$ & $1.499(5)$ \\
\hline C8A-C9A & $1.492(7)$ & $\mathrm{N} 4 \mathrm{C}-\mathrm{C} 8 \mathrm{C}$ & $1.486(6)$ \\
\hline $\mathrm{C} 8 \mathrm{~A}-\mathrm{H} 8 \mathrm{~A}$ & 0.9900 & $\mathrm{~N} 4 \mathrm{C}-\mathrm{C} 7 \mathrm{C}$ & $1.488(6)$ \\
\hline $\mathrm{C} 8 \mathrm{~A}-\mathrm{H} 8 \mathrm{~B}$ & 0.9900 & $\mathrm{~N} 4 \mathrm{C}-\mathrm{C} 16 \mathrm{C}$ & $1.503(5)$ \\
\hline C9A-H9A & 0.9900 & $\mathrm{C} 1 \mathrm{C}-\mathrm{C} 2 \mathrm{C}$ & $1.509(6)$ \\
\hline C9A-H9B & 0.9900 & $\mathrm{C} 1 \mathrm{C}-\mathrm{H} 1 \mathrm{E}$ & 0.9900 \\
\hline $\mathrm{C} 10 \mathrm{~A}-\mathrm{C} 11 \mathrm{~A}$ & $1.514(6)$ & $\mathrm{C} 1 \mathrm{C}-\mathrm{H} 1 \mathrm{~F}$ & 0.9900 \\
\hline C10A-H10A & 0.9900 & $\mathrm{C} 2 \mathrm{C}-\mathrm{C} 3 \mathrm{C}$ & $1.527(6)$ \\
\hline C10A-H10B & 0.9900 & $\mathrm{C} 2 \mathrm{C}-\mathrm{H} 2 \mathrm{E}$ & 0.9900 \\
\hline C12A-C13A & $1.508(6)$ & $\mathrm{C} 2 \mathrm{C}-\mathrm{H} 2 \mathrm{~F}$ & 0.9900 \\
\hline C12A-H12A & 0.9900 & CЗС-H3E & 0.9900 \\
\hline C12A-H12B & 0.9900 & $\mathrm{C} 3 \mathrm{C}-\mathrm{H} 3 \mathrm{~F}$ & 0.9900 \\
\hline C14A-C15A & $1.497(6)$ & $C 4 C-C 5 C$ & $1.501(6)$ \\
\hline $\mathrm{C} 14 \mathrm{~A}-\mathrm{H} 14 \mathrm{~A}$ & 0.9900 & $\mathrm{C} 4 \mathrm{C}-\mathrm{H} 4 \mathrm{E}$ & 0.9900 \\
\hline C14A-H14B & 0.9900 & $\mathrm{C} 4 \mathrm{C}-\mathrm{H} 4 \mathrm{~F}$ & 0.9900 \\
\hline C16A-C17A & $1.518(6)$ & C5C-H5E & 0.9900 \\
\hline $\mathrm{C} 16 \mathrm{~A}-\mathrm{H} 16 \mathrm{~A}$ & 0.9900 & $\mathrm{C} 5 \mathrm{C}-\mathrm{H} 5 \mathrm{~F}$ & 0.9900 \\
\hline C16A-H16B & 0.9900 & $\mathrm{C} 6 \mathrm{C}-\mathrm{C} 7 \mathrm{C}$ & $1.502(7)$ \\
\hline Zr2-O1B & $2.103(3)$ & C6C-H6E & 0.9900 \\
\hline Zr2-O5B & $2.114(3)$ & $\mathrm{C} 6 \mathrm{C}-\mathrm{H} 6 \mathrm{~F}$ & 0.9900 \\
\hline $\mathrm{Zr} 2-\mathrm{O} 3 \mathrm{~B}$ & $2.114(3)$ & C7C-H7E & 0.9900 \\
\hline Zr2-O7B & 2.117 (3) & C7C-H7F & 0.9900 \\
\hline Zr2-N2B & $2.440(4)$ & $\mathrm{C} 8 \mathrm{C}-\mathrm{C} 9 \mathrm{C}$ & $1.493(6)$ \\
\hline $\mathrm{Zr} 2-\mathrm{N} 4 \mathrm{~B}$ & $2.453(4)$ & $\mathrm{C} 8 \mathrm{C}-\mathrm{H} 8 \mathrm{E}$ & 0.9900 \\
\hline
\end{tabular}




\begin{tabular}{|c|c|c|c|}
\hline $\mathrm{Zr} 2-\mathrm{N} 3 \mathrm{~B}$ & $2.476(4)$ & $\mathrm{C} 8 \mathrm{C}-\mathrm{H} 8 \mathrm{~F}$ & 0.9900 \\
\hline Zr2-N1B & $2.513(4)$ & C9C-H9E & 0.9900 \\
\hline O1B-C11B & $1.312(6)$ & $\mathrm{C} 9 \mathrm{C}-\mathrm{H} 9 \mathrm{~F}$ & 0.9900 \\
\hline $\mathrm{O} 2 \mathrm{~B}-\mathrm{C} 11 \mathrm{~B}$ & $1.208(5)$ & C10C-C11C & $1.512(6)$ \\
\hline O3B-C13B & $1.297(6)$ & C10C-H10E & 0.9900 \\
\hline O4B-C13B & $1.217(6)$ & $\mathrm{C} 10 \mathrm{C}-\mathrm{H} 10 \mathrm{~F}$ & 0.9900 \\
\hline O5B-C15B & $1.298(5)$ & $\mathrm{C} 12 \mathrm{C}-\mathrm{C} 13 \mathrm{C}$ & $1.507(6)$ \\
\hline O6B-C15B & $1.226(5)$ & C12C-H12E & 0.9900 \\
\hline O7B-C17B & $1.296(6)$ & $\mathrm{C} 12 \mathrm{C}-\mathrm{H} 12 \mathrm{~F}$ & 0.9900 \\
\hline O8B-C17B & $1.218(6)$ & C14C-C15C & $1.508(6)$ \\
\hline N1B-C10B & $1.491(6)$ & $\mathrm{C} 14 \mathrm{C}-\mathrm{H} 14 \mathrm{E}$ & 0.9900 \\
\hline N1B-C1B & $1.496(6)$ & $\mathrm{C} 14 \mathrm{C}-\mathrm{H} 14 \mathrm{~F}$ & 0.9900 \\
\hline N1B-C9B & $1.527(6)$ & $\mathrm{C} 16 \mathrm{C}-\mathrm{C} 17 \mathrm{C}$ & $1.510(7)$ \\
\hline $\mathrm{N} 2 \mathrm{~B}-\mathrm{C} 3 \mathrm{~B}$ & $1.492(6)$ & C16C-H16E & 0.9900 \\
\hline $\mathrm{N} 2 \mathrm{~B}-\mathrm{C} 12 \mathrm{~B}$ & $1.496(6)$ & $\mathrm{C} 16 \mathrm{C}-\mathrm{H} 16 \mathrm{~F}$ & 0.9900 \\
\hline $\mathrm{N} 2 \mathrm{~B}-\mathrm{C} 4 \mathrm{~B}$ & $1.513(6)$ & $\mathrm{O} 1-\mathrm{H} 11$ & $0.818(14)$ \\
\hline N3B-C14B & $1.471(6)$ & $\mathrm{O} 1-\mathrm{H} 12$ & $0.818(14)$ \\
\hline N3B-C5B & $1.479(6)$ & $\mathrm{O} 2-\mathrm{H} 21$ & $0.825(15)$ \\
\hline N3B-C6B & $1.506(6)$ & $\mathrm{O} 2-\mathrm{H} 22$ & $0.824(15)$ \\
\hline N4B-C8B & $1.492(6)$ & $\mathrm{O} 3-\mathrm{H} 31$ & $0.814(15)$ \\
\hline $\mathrm{N} 4 \mathrm{~B}-\mathrm{C} 16 \mathrm{~B}$ & $1.507(6)$ & O3-H32 & $0.814(14)$ \\
\hline N4B-C7B & $1.514(7)$ & $\mathrm{O} 4-\mathrm{H} 41$ & $0.822(15)$ \\
\hline C1B-C2B & $1.503(8)$ & $\mathrm{O} 4-\mathrm{H} 42$ & $0.820(15)$ \\
\hline $\mathrm{C} 1 \mathrm{~B}-\mathrm{H} 1 \mathrm{C}$ & 0.9900 & O5-H51 & $0.813(14)$ \\
\hline C1B-H1D & 0.9900 & $\mathrm{O} 5-\mathrm{H} 52$ & $0.817(14)$ \\
\hline $\mathrm{C} 2 \mathrm{~B}-\mathrm{C} 3 \mathrm{~B}$ & $1.492(8)$ & $\mathrm{O} 6-\mathrm{H} 61$ & $0.816(15)$ \\
\hline $\mathrm{C} 2 \mathrm{~B}-\mathrm{H} 2 \mathrm{C}$ & 0.9900 & $\mathrm{O} 6-\mathrm{H} 62$ & $0.814(14)$ \\
\hline $\mathrm{C} 2 \mathrm{~B}-\mathrm{H} 2 \mathrm{D}$ & 0.9900 & O7-H71 & $0.826(14)$ \\
\hline С $3 \mathrm{~B}-\mathrm{H} 3 \mathrm{C}$ & 0.9900 & O7-H72 & $0.823(15)$ \\
\hline
\end{tabular}




\begin{tabular}{|c|c|c|c|}
\hline C3B-H3D & 0.9900 & $\mathrm{O} 8-\mathrm{H} 81$ & $0.810(15)$ \\
\hline C4B-C5B & $1.465(7)$ & O8-H82 & $0.816(14)$ \\
\hline $\mathrm{C} 4 \mathrm{~B}-\mathrm{H} 4 \mathrm{C}$ & 0.9900 & O9-H91 & $0.826(14)$ \\
\hline $\mathrm{C} 4 \mathrm{~B}-\mathrm{H} 4 \mathrm{D}$ & 0.9900 & O9-H92 & $0.822(14)$ \\
\hline $\mathrm{C} 5 \mathrm{~B}-\mathrm{H} 5 \mathrm{C}$ & 0.9900 & $\mathrm{O} 10-\mathrm{H} 101$ & $0.814(14)$ \\
\hline C5B-H5D & 0.9900 & $\mathrm{O} 10-\mathrm{H} 102$ & $0.815(15)$ \\
\hline C6B-C7B & $1.471(7)$ & N2B-C4B-H4D & 109.4 \\
\hline O1A-Zr1-O5A & 114.05 (12) & $\mathrm{H} 4 \mathrm{C}-\mathrm{C} 4 \mathrm{~B}-\mathrm{H} 4 \mathrm{D}$ & 108.0 \\
\hline $\mathrm{O} 1 \mathrm{~A}-\mathrm{Zr} 1-\mathrm{O} 7 \mathrm{~A}$ & $75.02(12)$ & $\mathrm{C} 4 \mathrm{~B}-\mathrm{C} 5 \mathrm{~B}-\mathrm{N} 3 \mathrm{~B}$ & $110.4(4)$ \\
\hline O5A-Zr1-O7A & $79.88(12)$ & $\mathrm{C} 4 \mathrm{~B}-\mathrm{C} 5 \mathrm{~B}-\mathrm{H} 5 \mathrm{C}$ & 109.6 \\
\hline $01 \mathrm{~A}-\mathrm{Zr} 1-\mathrm{O} 3 \mathrm{~A}$ & $76.12(11)$ & N3B-C5B-H5C & 109.6 \\
\hline O5A-Zr1-O3A & 75.81 (12) & C4B-C5B-H5D & 109.6 \\
\hline O7A-Zr1-O3A & $129.97(12)$ & N3B-C5B-H5D & 109.6 \\
\hline $\mathrm{O} 1 \mathrm{~A}-\mathrm{Zr} 1-\mathrm{N} 4 \mathrm{~A}$ & $89.25(12)$ & $\mathrm{H} 5 \mathrm{C}-\mathrm{C} 5 \mathrm{~B}-\mathrm{H} 5 \mathrm{D}$ & 108.1 \\
\hline $\mathrm{O} 5 \mathrm{~A}-\mathrm{Zr} 1-\mathrm{N} 4 \mathrm{~A}$ & $137.09(12)$ & C7B-C6B-N3B & $110.4(4)$ \\
\hline $\mathrm{O} 7 \mathrm{~A}-\mathrm{Zr} 1-\mathrm{N} 4 \mathrm{~A}$ & $71.80(12)$ & C7B-C6B-H6C & 109.6 \\
\hline $\mathrm{O} 3 \mathrm{~A}-\mathrm{Zr} 1-\mathrm{N} 4 \mathrm{~A}$ & $146.86(12)$ & N3B-C6B-H6C & 109.6 \\
\hline $\mathrm{O} 1 \mathrm{~A}-\mathrm{Zr} 1-\mathrm{N} 2 \mathrm{~A}$ & $141.02(12)$ & C7B-C6B-H6D & 109.6 \\
\hline $\mathrm{O} 5 \mathrm{~A}-\mathrm{Zr} 1-\mathrm{N} 2 \mathrm{~A}$ & 76.30 (12) & N3B-C6B-H6D & 109.6 \\
\hline $\mathrm{O} 7 \mathrm{~A}-\mathrm{Zr} 1-\mathrm{N} 2 \mathrm{~A}$ & $142.97(12)$ & $\mathrm{H} 6 \mathrm{C}-\mathrm{C} 6 \mathrm{~B}-\mathrm{H} 6 \mathrm{D}$ & 108.1 \\
\hline $\mathrm{O} 3 \mathrm{~A}-\mathrm{Zr} 1-\mathrm{N} 2 \mathrm{~A}$ & $70.22(11)$ & $\mathrm{C} 6 \mathrm{~B}-\mathrm{C} 7 \mathrm{~B}-\mathrm{N} 4 \mathrm{~B}$ & $111.9(4)$ \\
\hline $\mathrm{N} 4 \mathrm{~A}-\mathrm{Zr} 1-\mathrm{N} 2 \mathrm{~A}$ & $108.49(12)$ & C6B-C7B-H7C & 109.2 \\
\hline $\mathrm{O} 1 \mathrm{~A}-\mathrm{Zr} 1-\mathrm{N} 3 \mathrm{~A}$ & $146.55(12)$ & N4B-C7B-H7C & 109.2 \\
\hline O5A-Zr1-N3A & $71.23(12)$ & C6B-C7B-H7D & 109.2 \\
\hline O7A-Zr1-N3A & 73.59 (12) & N4B-C7B-H7D & 109.2 \\
\hline $\mathrm{O} 3 \mathrm{~A}-\mathrm{Zr} 1-\mathrm{N} 3 \mathrm{~A}$ & $134.49(11)$ & $\mathrm{H} 7 \mathrm{C}-\mathrm{C} 7 \mathrm{~B}-\mathrm{H} 7 \mathrm{D}$ & 107.9 \\
\hline $\mathrm{N} 4 \mathrm{~A}-\mathrm{Zr} 1-\mathrm{N} 3 \mathrm{~A}$ & $70.23(12)$ & $\mathrm{C} 9 \mathrm{~B}-\mathrm{C} 8 \mathrm{~B}-\mathrm{N} 4 \mathrm{~B}$ & $109.9(4)$ \\
\hline $\mathrm{N} 2 \mathrm{~A}-\mathrm{Zr} 1-\mathrm{N} 3 \mathrm{~A}$ & $72.11(12)$ & $\mathrm{C} 9 \mathrm{~B}-\mathrm{C} 8 \mathrm{~B}-\mathrm{H} 8 \mathrm{C}$ & 109.7 \\
\hline $\mathrm{O} 1 \mathrm{~A}-\mathrm{Zr} 1-\mathrm{N} 1 \mathrm{~A}$ & $67.82(12)$ & $\mathrm{N} 4 \mathrm{~B}-\mathrm{C} 8 \mathrm{~B}-\mathrm{H} 8 \mathrm{C}$ & 109.7 \\
\hline
\end{tabular}




\begin{tabular}{|c|c|c|c|}
\hline $\mathrm{O} 5 \mathrm{~A}-\mathrm{Zr} 1-\mathrm{N} 1 \mathrm{~A}$ & $150.57(12)$ & C9B-C8B-H8D & 109.7 \\
\hline O7A-Zr1-N1A & $126.40(11)$ & N4B-C8B-H8D & 109.7 \\
\hline $\mathrm{O} 3 \mathrm{~A}-\mathrm{Zr} 1-\mathrm{N} 1 \mathrm{~A}$ & $76.41(12)$ & $\mathrm{H} 8 \mathrm{C}-\mathrm{C} 8 \mathrm{~B}-\mathrm{H} 8 \mathrm{D}$ & 108.2 \\
\hline $\mathrm{N} 4 \mathrm{~A}-\mathrm{Zr} 1-\mathrm{N} 1 \mathrm{~A}$ & $70.53(12)$ & C8B-C9B-N1B & $110.6(4)$ \\
\hline $\mathrm{N} 2 \mathrm{~A}-\mathrm{Zr} 1-\mathrm{N} 1 \mathrm{~A}$ & $85.35(12)$ & C8B-C9B-H9C & 109.5 \\
\hline $\mathrm{N} 3 \mathrm{~A}-\mathrm{Zr} 1-\mathrm{N} 1 \mathrm{~A}$ & $124.72(12)$ & N1B-C9B-H9C & 109.5 \\
\hline $\mathrm{C} 11 \mathrm{~A}-\mathrm{O} 1 \mathrm{~A}-\mathrm{Zr} 1$ & $122.5(3)$ & C8B-C9B-H9D & 109.5 \\
\hline C13A-O3A-Zr1 & $126.4(3)$ & N1B-C9B-H9D & 109.5 \\
\hline C15A-O5A-Zr1 & $126.2(3)$ & $\mathrm{H} 9 \mathrm{C}-\mathrm{C} 9 \mathrm{~B}-\mathrm{H} 9 \mathrm{D}$ & 108.1 \\
\hline C17A-O7A-Zr1 & $121.0(3)$ & N1B-C10B-C11B & $110.2(4)$ \\
\hline $\mathrm{C} 1 \mathrm{~A}-\mathrm{N} 1 \mathrm{~A}-\mathrm{C} 10 \mathrm{~A}$ & $108.9(4)$ & $\mathrm{N} 1 \mathrm{~B}-\mathrm{C} 10 \mathrm{~B}-\mathrm{H} 10 \mathrm{C}$ & 109.6 \\
\hline $\mathrm{C} 1 \mathrm{~A}-\mathrm{N} 1 \mathrm{~A}-\mathrm{C} 9 \mathrm{~A}$ & $107.2(3)$ & $\mathrm{C} 11 \mathrm{~B}-\mathrm{C} 10 \mathrm{~B}-\mathrm{H} 10 \mathrm{C}$ & 109.6 \\
\hline C10A-N1A-C9A & $105.6(3)$ & N1B-C10B-H10D & 109.6 \\
\hline $\mathrm{C} 1 \mathrm{~A}-\mathrm{N} 1 \mathrm{~A}-\mathrm{Zr} 1$ & $123.6(3)$ & C11B-C10B-H10D & 109.6 \\
\hline $\mathrm{C} 10 \mathrm{~A}-\mathrm{N} 1 \mathrm{~A}-\mathrm{Zr} 1$ & $103.1(2)$ & $\mathrm{H} 10 \mathrm{C}-\mathrm{C} 10 \mathrm{~B}-\mathrm{H} 10 \mathrm{D}$ & 108.1 \\
\hline $\mathrm{C} 9 \mathrm{~A}-\mathrm{N} 1 \mathrm{~A}-\mathrm{Zr} 1$ & $107.2(2)$ & $\mathrm{O} 2 \mathrm{~B}-\mathrm{C} 11 \mathrm{~B}-\mathrm{O} 1 \mathrm{~B}$ & $123.9(5)$ \\
\hline $\mathrm{C} 12 \mathrm{~A}-\mathrm{N} 2 \mathrm{~A}-\mathrm{C} 4 \mathrm{~A}$ & $108.1(3)$ & O2B-C11B-C10B & $121.9(4)$ \\
\hline $\mathrm{C} 12 \mathrm{~A}-\mathrm{N} 2 \mathrm{~A}-\mathrm{C} 3 \mathrm{~A}$ & $107.7(3)$ & O1B-C11B-C10B & $114.2(4)$ \\
\hline $\mathrm{C} 4 \mathrm{~A}-\mathrm{N} 2 \mathrm{~A}-\mathrm{C} 3 \mathrm{~A}$ & $108.0(3)$ & $\mathrm{C} 13 \mathrm{~B}-\mathrm{C} 12 \mathrm{~B}-\mathrm{N} 2 \mathrm{~B}$ & $112.6(4)$ \\
\hline $\mathrm{C} 12 \mathrm{~A}-\mathrm{N} 2 \mathrm{~A}-\mathrm{Zr} 1$ & $107.7(3)$ & $\mathrm{C} 13 \mathrm{~B}-\mathrm{C} 12 \mathrm{~B}-\mathrm{H} 12 \mathrm{C}$ & 109.1 \\
\hline $\mathrm{C} 4 \mathrm{~A}-\mathrm{N} 2 \mathrm{~A}-\mathrm{Zr} 1$ & $110.7(2)$ & $\mathrm{N} 2 \mathrm{~B}-\mathrm{C} 12 \mathrm{~B}-\mathrm{H} 12 \mathrm{C}$ & 109.1 \\
\hline $\mathrm{C} 3 \mathrm{~A}-\mathrm{N} 2 \mathrm{~A}-\mathrm{Zr} 1$ & $114.4(3)$ & $\mathrm{C} 13 \mathrm{~B}-\mathrm{C} 12 \mathrm{~B}-\mathrm{H} 12 \mathrm{D}$ & 109.1 \\
\hline $\mathrm{C} 14 \mathrm{~A}-\mathrm{N} 3 \mathrm{~A}-\mathrm{C} 6 \mathrm{~A}$ & $109.7(3)$ & $\mathrm{N} 2 \mathrm{~B}-\mathrm{C} 12 \mathrm{~B}-\mathrm{H} 12 \mathrm{D}$ & 109.1 \\
\hline C14A-N3A-C5A & $109.3(3)$ & $\mathrm{H} 12 \mathrm{C}-\mathrm{C} 12 \mathrm{~B}-\mathrm{H} 12 \mathrm{D}$ & 107.8 \\
\hline $\mathrm{C} 6 \mathrm{~A}-\mathrm{N} 3 \mathrm{~A}-\mathrm{C} 5 \mathrm{~A}$ & $108.3(3)$ & O4B-C13B-O3B & $123.7(5)$ \\
\hline C14A-N3A-Zr1 & $106.8(3)$ & O4B-C13B-C12B & $120.4(5)$ \\
\hline $\mathrm{C} 6 \mathrm{~A}-\mathrm{N} 3 \mathrm{~A}-\mathrm{Zr} 1$ & $112.6(3)$ & O3B-C13B-C12B & $115.9(4)$ \\
\hline $\mathrm{C} 5 \mathrm{~A}-\mathrm{N} 3 \mathrm{~A}-\mathrm{Zr} 1$ & $110.1(3)$ & N3B-C14B-C15B & $113.0(4)$ \\
\hline $\mathrm{C} 16 \mathrm{~A}-\mathrm{N} 4 \mathrm{~A}-\mathrm{C} 7 \mathrm{~A}$ & $109.5(4)$ & N3B-C14B-H14C & 109.0 \\
\hline
\end{tabular}




\begin{tabular}{|c|c|c|c|}
\hline $\mathrm{C} 16 \mathrm{~A}-\mathrm{N} 4 \mathrm{~A}-\mathrm{C} 8 \mathrm{~A}$ & $108.9(3)$ & C15B-C14B-H14C & 109.0 \\
\hline $\mathrm{C} 7 \mathrm{~A}-\mathrm{N} 4 \mathrm{~A}-\mathrm{C} 8 \mathrm{~A}$ & $107.4(3)$ & N3B-C14B-H14D & 109.0 \\
\hline $\mathrm{C} 16 \mathrm{~A}-\mathrm{N} 4 \mathrm{~A}-\mathrm{Zr} 1$ & $103.9(2)$ & C15B-C14B-H14D & 109.0 \\
\hline $\mathrm{C} 7 \mathrm{~A}-\mathrm{N} 4 \mathrm{~A}-\mathrm{Zr} 1$ & $113.2(2)$ & $\mathrm{H} 14 \mathrm{C}-\mathrm{C} 14 \mathrm{~B}-\mathrm{H} 14 \mathrm{D}$ & 107.8 \\
\hline $\mathrm{C} 8 \mathrm{~A}-\mathrm{N} 4 \mathrm{~A}-\mathrm{Zr} 1$ & $113.8(3)$ & O6B-C15B-O5B & $123.0(4)$ \\
\hline $\mathrm{N} 1 \mathrm{~A}-\mathrm{C} 1 \mathrm{~A}-\mathrm{C} 2 \mathrm{~A}$ & $115.2(4)$ & O6B-C15B-C14B & $120.4(4)$ \\
\hline $\mathrm{N} 1 \mathrm{~A}-\mathrm{C} 1 \mathrm{~A}-\mathrm{H} 1 \mathrm{~A}$ & 108.5 & O5B-C15B-C14B & $116.5(4)$ \\
\hline $\mathrm{C} 2 \mathrm{~A}-\mathrm{C} 1 \mathrm{~A}-\mathrm{H} 1 \mathrm{~A}$ & 108.5 & C17B-C16B-N4B & $111.9(4)$ \\
\hline $\mathrm{N} 1 \mathrm{~A}-\mathrm{C} 1 \mathrm{~A}-\mathrm{H} 1 \mathrm{~B}$ & 108.5 & C17B-C16B-H16C & 109.2 \\
\hline $\mathrm{C} 2 \mathrm{~A}-\mathrm{C} 1 \mathrm{~A}-\mathrm{H} 1 \mathrm{~B}$ & 108.5 & $\mathrm{~N} 4 \mathrm{~B}-\mathrm{C} 16 \mathrm{~B}-\mathrm{H} 16 \mathrm{C}$ & 109.2 \\
\hline $\mathrm{H} 1 \mathrm{~A}-\mathrm{C} 1 \mathrm{~A}-\mathrm{H} 1 \mathrm{~B}$ & 107.5 & C17B-C16B-H16D & 109.2 \\
\hline $\mathrm{C} 3 \mathrm{~A}-\mathrm{C} 2 \mathrm{~A}-\mathrm{C} 1 \mathrm{~A}$ & $114.0(4)$ & N4B-C16B-H16D & 109.2 \\
\hline $\mathrm{C} 3 \mathrm{~A}-\mathrm{C} 2 \mathrm{~A}-\mathrm{H} 2 \mathrm{~A}$ & 108.8 & $\mathrm{H} 16 \mathrm{C}-\mathrm{C} 16 \mathrm{~B}-\mathrm{H} 16 \mathrm{D}$ & 107.9 \\
\hline $\mathrm{C} 1 \mathrm{~A}-\mathrm{C} 2 \mathrm{~A}-\mathrm{H} 2 \mathrm{~A}$ & 108.8 & O8B-C17B-O7B & $123.2(5)$ \\
\hline $\mathrm{C} 3 \mathrm{~A}-\mathrm{C} 2 \mathrm{~A}-\mathrm{H} 2 \mathrm{~B}$ & 108.8 & O8B-C17B-C16B & $120.5(5)$ \\
\hline $\mathrm{C} 1 \mathrm{~A}-\mathrm{C} 2 \mathrm{~A}-\mathrm{H} 2 \mathrm{~B}$ & 108.8 & O7B-C17B-C16B & $116.3(4)$ \\
\hline $\mathrm{H} 2 \mathrm{~A}-\mathrm{C} 2 \mathrm{~A}-\mathrm{H} 2 \mathrm{~B}$ & 107.6 & O1C-Zr3-O5C & $113.92(12)$ \\
\hline $\mathrm{C} 2 \mathrm{~A}-\mathrm{C} 3 \mathrm{~A}-\mathrm{N} 2 \mathrm{~A}$ & $116.5(4)$ & O1C-Zr3-O7C & $74.97(12)$ \\
\hline $\mathrm{C} 2 \mathrm{~A}-\mathrm{C} 3 \mathrm{~A}-\mathrm{H} 3 \mathrm{~A}$ & 108.2 & O5C-Zr3-O7C & $79.40(12)$ \\
\hline $\mathrm{N} 2 \mathrm{~A}-\mathrm{C} 3 \mathrm{~A}-\mathrm{H} 3 \mathrm{~A}$ & 108.2 & $\mathrm{O} 1 \mathrm{C}-\mathrm{Zr} 3-\mathrm{O} 3 \mathrm{C}$ & $76.07(11)$ \\
\hline $\mathrm{C} 2 \mathrm{~A}-\mathrm{C} 3 \mathrm{~A}-\mathrm{H} 3 \mathrm{~B}$ & 108.2 & $\mathrm{O} 5 \mathrm{C}-\mathrm{Zr} 3-\mathrm{O} 3 \mathrm{C}$ & $75.97(11)$ \\
\hline $\mathrm{N} 2 \mathrm{~A}-\mathrm{C} 3 \mathrm{~A}-\mathrm{H} 3 \mathrm{~B}$ & 108.2 & $\mathrm{O} 7 \mathrm{C}-\mathrm{Zr} 3-\mathrm{O} 3 \mathrm{C}$ & $129.64(12)$ \\
\hline $\mathrm{H} 3 \mathrm{~A}-\mathrm{C} 3 \mathrm{~A}-\mathrm{H} 3 \mathrm{~B}$ & 107.3 & $\mathrm{O} 1 \mathrm{C}-\mathrm{Zr} 3-\mathrm{N} 4 \mathrm{C}$ & $88.99(11)$ \\
\hline $\mathrm{N} 2 \mathrm{~A}-\mathrm{C} 4 \mathrm{~A}-\mathrm{C} 5 \mathrm{~A}$ & $110.9(4)$ & $\mathrm{O} 5 \mathrm{C}-\mathrm{Zr} 3-\mathrm{N} 4 \mathrm{C}$ & $136.95(12)$ \\
\hline $\mathrm{N} 2 \mathrm{~A}-\mathrm{C} 4 \mathrm{~A}-\mathrm{H} 4 \mathrm{~A}$ & 109.5 & O7C-Zr3-N4C & $71.85(11)$ \\
\hline $\mathrm{C} 5 \mathrm{~A}-\mathrm{C} 4 \mathrm{~A}-\mathrm{H} 4 \mathrm{~A}$ & 109.5 & $\mathrm{O} 3 \mathrm{C}-\mathrm{Zr} 3-\mathrm{N} 4 \mathrm{C}$ & $146.90(12)$ \\
\hline $\mathrm{N} 2 \mathrm{~A}-\mathrm{C} 4 \mathrm{~A}-\mathrm{H} 4 \mathrm{~B}$ & 109.5 & $\mathrm{O} 1 \mathrm{C}-\mathrm{Zr} 3-\mathrm{N} 3 \mathrm{C}$ & $146.35(12)$ \\
\hline $\mathrm{C} 5 \mathrm{~A}-\mathrm{C} 4 \mathrm{~A}-\mathrm{H} 4 \mathrm{~B}$ & 109.5 & $\mathrm{O} 5 \mathrm{C}-\mathrm{Zr} 3-\mathrm{N} 3 \mathrm{C}$ & $71.17(11)$ \\
\hline $\mathrm{H} 4 \mathrm{~A}-\mathrm{C} 4 \mathrm{~A}-\mathrm{H} 4 \mathrm{~B}$ & 108.0 & O7C-Zr3-N3C & $73.48(12)$ \\
\hline
\end{tabular}




\begin{tabular}{|c|c|c|c|}
\hline $\mathrm{N} 3 \mathrm{~A}-\mathrm{C} 5 \mathrm{~A}-\mathrm{C} 4 \mathrm{~A}$ & $110.9(4)$ & $\mathrm{O} 3 \mathrm{C}-\mathrm{Zr} 3-\mathrm{N} 3 \mathrm{C}$ & $134.66(11)$ \\
\hline N3A-C5A-H5A & 109.5 & $\mathrm{~N} 4 \mathrm{C}-\mathrm{Zr} 3-\mathrm{N} 3 \mathrm{C}$ & 70.35 (12) \\
\hline $\mathrm{C} 4 \mathrm{~A}-\mathrm{C} 5 \mathrm{~A}-\mathrm{H} 5 \mathrm{~A}$ & 109.5 & $\mathrm{O} 1 \mathrm{C}-\mathrm{Zr} 3-\mathrm{N} 2 \mathrm{C}$ & $141.19(12)$ \\
\hline N3A-C5A-H5B & 109.5 & $\mathrm{O} 5 \mathrm{C}-\mathrm{Zr} 3-\mathrm{N} 2 \mathrm{C}$ & $76.71(12)$ \\
\hline $\mathrm{C} 4 \mathrm{~A}-\mathrm{C} 5 \mathrm{~A}-\mathrm{H} 5 \mathrm{~B}$ & 109.5 & $\mathrm{O} 7 \mathrm{C}-\mathrm{Zr} 3-\mathrm{N} 2 \mathrm{C}$ & $142.87(12)$ \\
\hline $\mathrm{H} 5 \mathrm{~A}-\mathrm{C} 5 \mathrm{~A}-\mathrm{H} 5 \mathrm{~B}$ & 108.0 & $\mathrm{O} 3 \mathrm{C}-\mathrm{Zr} 3-\mathrm{N} 2 \mathrm{C}$ & $70.55(11)$ \\
\hline $\mathrm{C} 7 \mathrm{~A}-\mathrm{C} 6 \mathrm{~A}-\mathrm{N} 3 \mathrm{~A}$ & $108.5(4)$ & $\mathrm{N} 4 \mathrm{C}-\mathrm{Zr} 3-\mathrm{N} 2 \mathrm{C}$ & $108.52(11)$ \\
\hline $\mathrm{C} 7 \mathrm{~A}-\mathrm{C} 6 \mathrm{~A}-\mathrm{H} 6 \mathrm{~A}$ & 110.0 & $\mathrm{~N} 3 \mathrm{C}-\mathrm{Zr} 3-\mathrm{N} 2 \mathrm{C}$ & $72.11(12)$ \\
\hline $\mathrm{N} 3 \mathrm{~A}-\mathrm{C} 6 \mathrm{~A}-\mathrm{H} 6 \mathrm{~A}$ & 110.0 & $\mathrm{O} 1 \mathrm{C}-\mathrm{Zr3}-\mathrm{N} 1 \mathrm{C}$ & $67.96(11)$ \\
\hline $\mathrm{C} 7 \mathrm{~A}-\mathrm{C} 6 \mathrm{~A}-\mathrm{H} 6 \mathrm{~B}$ & 110.0 & $\mathrm{O} 5 \mathrm{C}-\mathrm{Zr3}-\mathrm{N} 1 \mathrm{C}$ & $151.11(11)$ \\
\hline $\mathrm{N} 3 \mathrm{~A}-\mathrm{C} 6 \mathrm{~A}-\mathrm{H} 6 \mathrm{~B}$ & 110.0 & $\mathrm{O} 7 \mathrm{C}-\mathrm{Zr3}-\mathrm{N} 1 \mathrm{C}$ & $126.40(11)$ \\
\hline $\mathrm{H} 6 \mathrm{~A}-\mathrm{C} 6 \mathrm{~A}-\mathrm{H} 6 \mathrm{~B}$ & 108.4 & $\mathrm{O} 3 \mathrm{C}-\mathrm{Zr3}-\mathrm{N} 1 \mathrm{C}$ & $76.78(11)$ \\
\hline $\mathrm{C} 6 \mathrm{~A}-\mathrm{C} 7 \mathrm{~A}-\mathrm{N} 4 \mathrm{~A}$ & $111.5(4)$ & $\mathrm{N} 4 \mathrm{C}-\mathrm{Zr} 3-\mathrm{N} 1 \mathrm{C}$ & $70.22(11)$ \\
\hline $\mathrm{C} 6 \mathrm{~A}-\mathrm{C} 7 \mathrm{~A}-\mathrm{H} 7 \mathrm{~A}$ & 109.3 & $\mathrm{~N} 3 \mathrm{C}-\mathrm{Zr} 3-\mathrm{N} 1 \mathrm{C}$ & $124.54(11)$ \\
\hline $\mathrm{N} 4 \mathrm{~A}-\mathrm{C} 7 \mathrm{~A}-\mathrm{H} 7 \mathrm{~A}$ & 109.3 & $\mathrm{~N} 2 \mathrm{C}-\mathrm{Zr} 3-\mathrm{N} 1 \mathrm{C}$ & $85.35(11)$ \\
\hline $\mathrm{C} 6 \mathrm{~A}-\mathrm{C} 7 \mathrm{~A}-\mathrm{H} 7 \mathrm{~B}$ & 109.3 & $\mathrm{C} 11 \mathrm{C}-\mathrm{O} 1 \mathrm{C}-\mathrm{Zr3}$ & $123.0(3)$ \\
\hline $\mathrm{N} 4 \mathrm{~A}-\mathrm{C} 7 \mathrm{~A}-\mathrm{H} 7 \mathrm{~B}$ & 109.3 & $\mathrm{C} 13 \mathrm{C}-\mathrm{O} 3 \mathrm{C}-\mathrm{Zr} 3$ & $126.6(3)$ \\
\hline $\mathrm{H} 7 \mathrm{~A}-\mathrm{C} 7 \mathrm{~A}-\mathrm{H} 7 \mathrm{~B}$ & 108.0 & C15C-O5C-Zr3 & $126.5(3)$ \\
\hline $\mathrm{C} 9 \mathrm{~A}-\mathrm{C} 8 \mathrm{~A}-\mathrm{N} 4 \mathrm{~A}$ & 110.0 & C17C-O7C-Zr3 & $121.8(3)$ \\
\hline $\mathrm{C} 9 \mathrm{~A}-\mathrm{C} 8 \mathrm{~A}-\mathrm{H} 8 \mathrm{~A}$ & 109.7 & $\mathrm{C} 9 \mathrm{C}-\mathrm{N} 1 \mathrm{C}-\mathrm{C} 10 \mathrm{C}$ & $105.5(3)$ \\
\hline $\mathrm{N} 4 \mathrm{~A}-\mathrm{C} 8 \mathrm{~A}-\mathrm{H} 8 \mathrm{~A}$ & 109.7 & $\mathrm{C} 9 \mathrm{C}-\mathrm{N} 1 \mathrm{C}-\mathrm{C} 1 \mathrm{C}$ & $107.5(3)$ \\
\hline $\mathrm{C} 9 \mathrm{~A}-\mathrm{C} 8 \mathrm{~A}-\mathrm{H} 8 \mathrm{~B}$ & 109.7 & $\mathrm{C} 10 \mathrm{C}-\mathrm{N} 1 \mathrm{C}-\mathrm{C} 1 \mathrm{C}$ & 108.9 (3) \\
\hline $\mathrm{N} 4 \mathrm{~A}-\mathrm{C} 8 \mathrm{~A}-\mathrm{H} 8 \mathrm{~B}$ & 109.7 & $\mathrm{C} 9 \mathrm{C}-\mathrm{N} 1 \mathrm{C}-\mathrm{Zr3}$ & $107.5(2)$ \\
\hline $\mathrm{H} 8 \mathrm{~A}-\mathrm{C} 8 \mathrm{~A}-\mathrm{H} 8 \mathrm{~B}$ & 108.2 & $\mathrm{C} 10 \mathrm{C}-\mathrm{N} 1 \mathrm{C}-\mathrm{Zr3}$ & $102.7(3)$ \\
\hline $\mathrm{C} 8 \mathrm{~A}-\mathrm{C} 9 \mathrm{~A}-\mathrm{N} 1 \mathrm{~A}$ & $109.4(4)$ & $\mathrm{C} 1 \mathrm{C}-\mathrm{N} 1 \mathrm{C}-\mathrm{Zr3}$ & $123.5(3)$ \\
\hline $\mathrm{C} 8 \mathrm{~A}-\mathrm{C} 9 \mathrm{~A}-\mathrm{H} 9 \mathrm{~A}$ & 109.8 & $\mathrm{C} 4 \mathrm{C}-\mathrm{N} 2 \mathrm{C}-\mathrm{C} 12 \mathrm{C}$ & $107.8(3)$ \\
\hline $\mathrm{N} 1 \mathrm{~A}-\mathrm{C} 9 \mathrm{~A}-\mathrm{H} 9 \mathrm{~A}$ & 109.8 & $\mathrm{C} 4 \mathrm{C}-\mathrm{N} 2 \mathrm{C}-\mathrm{C} 3 \mathrm{C}$ & $107.9(3)$ \\
\hline $\mathrm{C} 8 \mathrm{~A}-\mathrm{C} 9 \mathrm{~A}-\mathrm{H} 9 \mathrm{~B}$ & 109.8 & $\mathrm{C} 12 \mathrm{C}-\mathrm{N} 2 \mathrm{C}-\mathrm{C} 3 \mathrm{C}$ & $108.6(3)$ \\
\hline $\mathrm{N} 1 \mathrm{~A}-\mathrm{C} 9 \mathrm{~A}-\mathrm{H} 9 \mathrm{~B}$ & 109.8 & $\mathrm{C} 4 \mathrm{C}-\mathrm{N} 2 \mathrm{C}-\mathrm{Zr} 3$ & $110.7(2)$ \\
\hline
\end{tabular}




\begin{tabular}{|c|c|c|c|}
\hline $\mathrm{H} 9 \mathrm{~A}-\mathrm{C} 9 \mathrm{~A}-\mathrm{H} 9 \mathrm{~B}$ & 108.2 & $\mathrm{C} 12 \mathrm{C}-\mathrm{N} 2 \mathrm{C}-\mathrm{Zr} 3$ & $106.9(2)$ \\
\hline $\mathrm{N} 1 \mathrm{~A}-\mathrm{C} 10 \mathrm{~A}-\mathrm{C} 11 \mathrm{~A}$ & $109.9(4)$ & $\mathrm{C} 3 \mathrm{C}-\mathrm{N} 2 \mathrm{C}-\mathrm{Zr} 3$ & $114.7(2)$ \\
\hline $\mathrm{N} 1 \mathrm{~A}-\mathrm{C} 10 \mathrm{~A}-\mathrm{H} 10 \mathrm{~A}$ & 109.7 & $\mathrm{C} 14 \mathrm{C}-\mathrm{N} 3 \mathrm{C}-\mathrm{C} 6 \mathrm{C}$ & $109.0(3)$ \\
\hline $\mathrm{C} 11 \mathrm{~A}-\mathrm{C} 10 \mathrm{~A}-\mathrm{H} 10 \mathrm{~A}$ & 109.7 & $\mathrm{C} 14 \mathrm{C}-\mathrm{N} 3 \mathrm{C}-\mathrm{C} 5 \mathrm{C}$ & $109.4(3)$ \\
\hline $\mathrm{N} 1 \mathrm{~A}-\mathrm{C} 10 \mathrm{~A}-\mathrm{H} 10 \mathrm{~B}$ & 109.7 & $\mathrm{C} 6 \mathrm{C}-\mathrm{N} 3 \mathrm{C}-\mathrm{C} 5 \mathrm{C}$ & $108.7(3)$ \\
\hline $\mathrm{C} 11 \mathrm{~A}-\mathrm{C} 10 \mathrm{~A}-\mathrm{H} 10 \mathrm{~B}$ & 109.7 & $\mathrm{C} 14 \mathrm{C}-\mathrm{N} 3 \mathrm{C}-\mathrm{Zr} 3$ & $107.2(3)$ \\
\hline $\mathrm{H} 10 \mathrm{~A}-\mathrm{C} 10 \mathrm{~A}-\mathrm{H} 10 \mathrm{~B}$ & 108.2 & $\mathrm{C} 6 \mathrm{C}-\mathrm{N} 3 \mathrm{C}-\mathrm{Zr} 3$ & $112.4(3)$ \\
\hline $\mathrm{O} 2 \mathrm{~A}-\mathrm{C} 11 \mathrm{~A}-\mathrm{O} 1 \mathrm{~A}$ & $123.8(4)$ & $\mathrm{C} 5 \mathrm{C}-\mathrm{N} 3 \mathrm{C}-\mathrm{Zr} 3$ & $110.1(2)$ \\
\hline $02 A-C 11 A-C 10 A$ & $120.4(4)$ & $\mathrm{C} 8 \mathrm{C}-\mathrm{N} 4 \mathrm{C}-\mathrm{C} 7 \mathrm{C}$ & $107.3(3)$ \\
\hline $01 A-C 11 A-C 10 A$ & $115.8(4)$ & $\mathrm{C} 8 \mathrm{C}-\mathrm{N} 4 \mathrm{C}-\mathrm{C} 16 \mathrm{C}$ & $109.0(3)$ \\
\hline $\mathrm{N} 2 \mathrm{~A}-\mathrm{C} 12 \mathrm{~A}-\mathrm{C} 13 \mathrm{~A}$ & $111.8(4)$ & $\mathrm{C} 7 \mathrm{C}-\mathrm{N} 4 \mathrm{C}-\mathrm{C} 16 \mathrm{C}$ & $109.2(3)$ \\
\hline $\mathrm{N} 2 \mathrm{~A}-\mathrm{C} 12 \mathrm{~A}-\mathrm{H} 12 \mathrm{~A}$ & 109.3 & $\mathrm{C} 8 \mathrm{C}-\mathrm{N} 4 \mathrm{C}-\mathrm{Zr} 3$ & $114.0(3)$ \\
\hline $\mathrm{C} 13 \mathrm{~A}-\mathrm{C} 12 \mathrm{~A}-\mathrm{H} 12 \mathrm{~A}$ & 109.3 & $\mathrm{C} 7 \mathrm{C}-\mathrm{N} 4 \mathrm{C}-\mathrm{Zr3}$ & $113.4(2)$ \\
\hline $\mathrm{N} 2 \mathrm{~A}-\mathrm{C} 12 \mathrm{~A}-\mathrm{H} 12 \mathrm{~B}$ & 109.3 & $\mathrm{C} 16 \mathrm{C}-\mathrm{N} 4 \mathrm{C}-\mathrm{Zr3}$ & $103.7(2)$ \\
\hline $\mathrm{C} 13 \mathrm{~A}-\mathrm{C} 12 \mathrm{~A}-\mathrm{H} 12 \mathrm{~B}$ & 109.3 & $\mathrm{~N} 1 \mathrm{C}-\mathrm{C} 1 \mathrm{C}-\mathrm{C} 2 \mathrm{C}$ & $115.2(4)$ \\
\hline $\mathrm{H} 12 \mathrm{~A}-\mathrm{C} 12 \mathrm{~A}-\mathrm{H} 12 \mathrm{~B}$ & 107.9 & $\mathrm{~N} 1 \mathrm{C}-\mathrm{C} 1 \mathrm{C}-\mathrm{H} 1 \mathrm{E}$ & 108.5 \\
\hline$O 4 A-C 13 A-O 3 A$ & $124.0(4)$ & $\mathrm{C} 2 \mathrm{C}-\mathrm{C} 1 \mathrm{C}-\mathrm{H} 1 \mathrm{E}$ & 108.5 \\
\hline O4A-C13A-C12A & $120.3(4)$ & $\mathrm{N} 1 \mathrm{C}-\mathrm{C} 1 \mathrm{C}-\mathrm{H} 1 \mathrm{~F}$ & 108.5 \\
\hline O3A-C13A-C12A & $115.7(4)$ & $\mathrm{C} 2 \mathrm{C}-\mathrm{C} 1 \mathrm{C}-\mathrm{H} 1 \mathrm{~F}$ & 108.5 \\
\hline N3A-C14A-C15A & $112.0(4)$ & $\mathrm{H} 1 \mathrm{E}-\mathrm{C} 1 \mathrm{C}-\mathrm{H} 1 \mathrm{~F}$ & 107.5 \\
\hline $\mathrm{N} 3 \mathrm{~A}-\mathrm{C} 14 \mathrm{~A}-\mathrm{H} 14 \mathrm{~A}$ & 109.2 & $\mathrm{C} 1 \mathrm{C}-\mathrm{C} 2 \mathrm{C}-\mathrm{C} 3 \mathrm{C}$ & $114.0(4)$ \\
\hline $\mathrm{C} 15 \mathrm{~A}-\mathrm{C} 14 \mathrm{~A}-\mathrm{H} 14 \mathrm{~A}$ & 109.2 & $\mathrm{C} 1 \mathrm{C}-\mathrm{C} 2 \mathrm{C}-\mathrm{H} 2 \mathrm{E}$ & 108.8 \\
\hline $\mathrm{N} 3 \mathrm{~A}-\mathrm{C} 14 \mathrm{~A}-\mathrm{H} 14 \mathrm{~B}$ & 109.2 & $\mathrm{C} 3 \mathrm{C}-\mathrm{C} 2 \mathrm{C}-\mathrm{H} 2 \mathrm{E}$ & 108.8 \\
\hline $\mathrm{C} 15 \mathrm{~A}-\mathrm{C} 14 \mathrm{~A}-\mathrm{H} 14 \mathrm{~B}$ & 109.2 & $\mathrm{C} 1 \mathrm{C}-\mathrm{C} 2 \mathrm{C}-\mathrm{H} 2 \mathrm{~F}$ & 108.8 \\
\hline $\mathrm{H} 14 \mathrm{~A}-\mathrm{C} 14 \mathrm{~A}-\mathrm{H} 14 \mathrm{~B}$ & 107.9 & $\mathrm{C} 3 \mathrm{C}-\mathrm{C} 2 \mathrm{C}-\mathrm{H} 2 \mathrm{~F}$ & 108.8 \\
\hline$O 6 A-C 15 A-O 5 A$ & $123.4(4)$ & $\mathrm{H} 2 \mathrm{E}-\mathrm{C} 2 \mathrm{C}-\mathrm{H} 2 \mathrm{~F}$ & 107.7 \\
\hline O6A-C15A-C14A & $120.9(4)$ & $\mathrm{N} 2 \mathrm{C}-\mathrm{C} 3 \mathrm{C}-\mathrm{C} 2 \mathrm{C}$ & $115.8(3)$ \\
\hline $05 A-C 15 A-C 14 A$ & $115.7(4)$ & $\mathrm{N} 2 \mathrm{C}-\mathrm{C} 3 \mathrm{C}-\mathrm{H} 3 \mathrm{E}$ & 108.3 \\
\hline $\mathrm{N} 4 \mathrm{~A}-\mathrm{C} 16 \mathrm{~A}-\mathrm{C} 17 \mathrm{~A}$ & $113.2(4)$ & $\mathrm{C} 2 \mathrm{C}-\mathrm{C} 3 \mathrm{C}-\mathrm{H} 3 \mathrm{E}$ & 108.3 \\
\hline
\end{tabular}




\begin{tabular}{|c|c|c|c|}
\hline $\mathrm{N} 4 \mathrm{~A}-\mathrm{C} 16 \mathrm{~A}-\mathrm{H} 16 \mathrm{~A}$ & 108.9 & $\mathrm{~N} 2 \mathrm{C}-\mathrm{C} 3 \mathrm{C}-\mathrm{H} 3 \mathrm{~F}$ & 108.3 \\
\hline C17A-C16A-H16A & 108.9 & $\mathrm{C} 2 \mathrm{C}-\mathrm{C} 3 \mathrm{C}-\mathrm{H} 3 \mathrm{~F}$ & 108.3 \\
\hline $\mathrm{N} 4 \mathrm{~A}-\mathrm{C} 16 \mathrm{~A}-\mathrm{H} 16 \mathrm{~B}$ & 108.9 & $\mathrm{H} 3 \mathrm{E}-\mathrm{C} 3 \mathrm{C}-\mathrm{H} 3 \mathrm{~F}$ & 107.4 \\
\hline $\mathrm{C} 17 \mathrm{~A}-\mathrm{C} 16 \mathrm{~A}-\mathrm{H} 16 \mathrm{~B}$ & 108.9 & $\mathrm{~N} 2 \mathrm{C}-\mathrm{C} 4 \mathrm{C}-\mathrm{C} 5 \mathrm{C}$ & $110.6(3)$ \\
\hline $\mathrm{H} 16 \mathrm{~A}-\mathrm{C} 16 \mathrm{~A}-\mathrm{H} 16 \mathrm{~B}$ & 107.7 & $\mathrm{~N} 2 \mathrm{C}-\mathrm{C} 4 \mathrm{C}-\mathrm{H} 4 \mathrm{E}$ & 109.5 \\
\hline$O 8 A-C 17 A-07 A$ & $124.6(5)$ & $\mathrm{C} 5 \mathrm{C}-\mathrm{C} 4 \mathrm{C}-\mathrm{H} 4 \mathrm{E}$ & 109.5 \\
\hline$O 8 A-C 17 A-C 16 A$ & $118.7(5)$ & $\mathrm{N} 2 \mathrm{C}-\mathrm{C} 4 \mathrm{C}-\mathrm{H} 4 \mathrm{~F}$ & 109.5 \\
\hline O7A-C17A-C16A & $116.7(4)$ & $\mathrm{C} 5 \mathrm{C}-\mathrm{C} 4 \mathrm{C}-\mathrm{H} 4 \mathrm{~F}$ & 109.5 \\
\hline O1B-Zr2-O5B & $114.01(11)$ & $\mathrm{H} 4 \mathrm{E}-\mathrm{C} 4 \mathrm{C}-\mathrm{H} 4 \mathrm{~F}$ & 108.1 \\
\hline O1B-Zr2-O3B & $75.83(12)$ & $\mathrm{N} 3 \mathrm{C}-\mathrm{C} 5 \mathrm{C}-\mathrm{C} 4 \mathrm{C}$ & $110.9(3)$ \\
\hline O5B-Zr2-O3B & 76.27 (12) & N3C-C5C-H5E & 109.5 \\
\hline O1B-Zr2-O7B & $75.59(12)$ & $\mathrm{C} 4 \mathrm{C}-\mathrm{C} 5 \mathrm{C}-\mathrm{H} 5 \mathrm{E}$ & 109.5 \\
\hline O5B-Zr2-O7B & $77.28(12)$ & $\mathrm{N} 3 \mathrm{C}-\mathrm{C} 5 \mathrm{C}-\mathrm{H} 5 \mathrm{~F}$ & 109.5 \\
\hline O3B-Zr2-O7B & $128.19(11)$ & $\mathrm{C} 4 \mathrm{C}-\mathrm{C} 5 \mathrm{C}-\mathrm{H} 5 \mathrm{~F}$ & 109.5 \\
\hline O1B-Zr2-N2B & $142.00(14)$ & $\mathrm{H} 5 \mathrm{E}-\mathrm{C} 5 \mathrm{C}-\mathrm{H} 5 \mathrm{~F}$ & 108.1 \\
\hline O5B-Zr2-N2B & $76.50(13)$ & $\mathrm{N} 3 \mathrm{C}-\mathrm{C} 6 \mathrm{C}-\mathrm{C} 7 \mathrm{C}$ & $108.6(4)$ \\
\hline $\mathrm{O} 3 \mathrm{~B}-\mathrm{Zr} 2-\mathrm{N} 2 \mathrm{~B}$ & $71.51(13)$ & N3C-C6C-H6E & 110.0 \\
\hline O7B-Zr2-N2B & $141.12(13)$ & C7C-C6C-H6E & 110.0 \\
\hline O1B-Zr2-N4B & $88.01(13)$ & $\mathrm{N} 3 \mathrm{C}-\mathrm{C} 6 \mathrm{C}-\mathrm{H} 6 \mathrm{~F}$ & 110.0 \\
\hline O5B-Zr2-N4B & $136.35(13)$ & $\mathrm{C} 7 \mathrm{C}-\mathrm{C} 6 \mathrm{C}-\mathrm{H} 6 \mathrm{~F}$ & 110.0 \\
\hline O3B-Zr2-N4B & $147.34(12)$ & $\mathrm{H} 6 \mathrm{E}-\mathrm{C} 6 \mathrm{C}-\mathrm{H} 6 \mathrm{~F}$ & 108.4 \\
\hline O7B-Zr2-N4B & $72.38(13)$ & $\mathrm{N} 4 \mathrm{C}-\mathrm{C} 7 \mathrm{C}-\mathrm{C} 6 \mathrm{C}$ & $111.2(3)$ \\
\hline $\mathrm{N} 2 \mathrm{~B}-\mathrm{Zr} 2-\mathrm{N} 4 \mathrm{~B}$ & $109.61(14)$ & N4C-C7C-H7E & 109.4 \\
\hline O1B-Zr2-N3B & $145.70(13)$ & C6C-C7C-H7E & 109.4 \\
\hline O5B-Zr2-N3B & 71.07 (12) & N4C-C7C-H7F & 109.4 \\
\hline O3B-Zr2-N3B & $135.41(12)$ & C6C-C7C-H7F & 109.4 \\
\hline O7B-Zr2-N3B & $72.65(13)$ & H7E-C7C-H7F & 108.0 \\
\hline $\mathrm{N} 2 \mathrm{~B}-\mathrm{Zr} 2-\mathrm{N} 3 \mathrm{~B}$ & $71.96(13)$ & $\mathrm{N} 4 \mathrm{C}-\mathrm{C} 8 \mathrm{C}-\mathrm{C} 9 \mathrm{C}$ & $110.2(3)$ \\
\hline N4B-Zr2-N3B & $70.39(13)$ & $\mathrm{N} 4 \mathrm{C}-\mathrm{C} 8 \mathrm{C}-\mathrm{H} 8 \mathrm{E}$ & 109.6 \\
\hline
\end{tabular}




\begin{tabular}{|c|c|c|c|}
\hline O1B-Zr2-N1B & $67.97(13)$ & $\mathrm{C} 9 \mathrm{C}-\mathrm{C} 8 \mathrm{C}-\mathrm{H} 8 \mathrm{E}$ & 109.6 \\
\hline O5B-Zr2-N1B & $151.43(14)$ & $\mathrm{N} 4 \mathrm{C}-\mathrm{C} 8 \mathrm{C}-\mathrm{H} 8 \mathrm{~F}$ & 109.6 \\
\hline O3B-Zr2-N1B & $76.86(13)$ & $\mathrm{C} 9 \mathrm{C}-\mathrm{C} 8 \mathrm{C}-\mathrm{H} 8 \mathrm{~F}$ & 109.6 \\
\hline O7B-Zr2-N1B & $128.13(13)$ & $\mathrm{H} 8 \mathrm{E}-\mathrm{C} 8 \mathrm{C}-\mathrm{H} 8 \mathrm{~F}$ & 108.1 \\
\hline N2B-Zr2-N1B & $85.93(13)$ & $\mathrm{N} 1 \mathrm{C}-\mathrm{C} 9 \mathrm{C}-\mathrm{C} 8 \mathrm{C}$ & $109.4(3)$ \\
\hline N4B-Zr2-N1B & $70.76(13)$ & $\mathrm{N} 1 \mathrm{C}-\mathrm{C} 9 \mathrm{C}-\mathrm{H} 9 \mathrm{E}$ & 109.8 \\
\hline N3B-Zr2-N1B & $124.67(13)$ & $\mathrm{C} 8 \mathrm{C}-\mathrm{C} 9 \mathrm{C}-\mathrm{H} 9 \mathrm{E}$ & 109.8 \\
\hline C11B-O1B-Zr2 & $123.9(3)$ & $\mathrm{N} 1 \mathrm{C}-\mathrm{C} 9 \mathrm{C}-\mathrm{H} 9 \mathrm{~F}$ & 109.8 \\
\hline $\mathrm{C} 13 \mathrm{~B}-\mathrm{O} 3 \mathrm{~B}-\mathrm{Zr} 2$ & $125.1(3)$ & $\mathrm{C} 8 \mathrm{C}-\mathrm{C} 9 \mathrm{C}-\mathrm{H} 9 \mathrm{~F}$ & 109.8 \\
\hline C15B-O5B-Zr2 & $125.6(3)$ & $\mathrm{H} 9 \mathrm{E}-\mathrm{C} 9 \mathrm{C}-\mathrm{H} 9 \mathrm{~F}$ & 108.2 \\
\hline C17B-O7B-Zr2 & $122.2(3)$ & $\mathrm{N} 1 \mathrm{C}-\mathrm{C} 10 \mathrm{C}-\mathrm{C} 11 \mathrm{C}$ & $110.6(4)$ \\
\hline $\mathrm{C} 10 \mathrm{~B}-\mathrm{N} 1 \mathrm{~B}-\mathrm{C} 1 \mathrm{~B}$ & $110.2(4)$ & $\mathrm{N} 1 \mathrm{C}-\mathrm{C} 10 \mathrm{C}-\mathrm{H} 10 \mathrm{E}$ & 109.5 \\
\hline $\mathrm{C} 10 \mathrm{~B}-\mathrm{N} 1 \mathrm{~B}-\mathrm{C} 9 \mathrm{~B}$ & $105.3(4)$ & $\mathrm{C} 11 \mathrm{C}-\mathrm{C} 10 \mathrm{C}-\mathrm{H} 10 \mathrm{E}$ & 109.5 \\
\hline C1B-N1B-C9B & $107.0(4)$ & $\mathrm{N} 1 \mathrm{C}-\mathrm{C} 10 \mathrm{C}-\mathrm{H} 10 \mathrm{~F}$ & 109.5 \\
\hline C10B-N1B-Zr2 & $103.3(3)$ & $\mathrm{C} 11 \mathrm{C}-\mathrm{C} 10 \mathrm{C}-\mathrm{H} 10 \mathrm{~F}$ & 109.5 \\
\hline $\mathrm{C} 1 \mathrm{~B}-\mathrm{N} 1 \mathrm{~B}-\mathrm{Zr} 2$ & $121.9(3)$ & $\mathrm{H} 10 \mathrm{E}-\mathrm{C} 10 \mathrm{C}-\mathrm{H} 10 \mathrm{~F}$ & 108.1 \\
\hline C9B-N1B-Zr2 & $108.1(3)$ & $\mathrm{O} 2 \mathrm{C}-\mathrm{C} 11 \mathrm{C}-\mathrm{O} 1 \mathrm{C}$ & $124.2(4)$ \\
\hline $\mathrm{C} 3 \mathrm{~B}-\mathrm{N} 2 \mathrm{~B}-\mathrm{C} 12 \mathrm{~B}$ & $107.6(4)$ & $\mathrm{O} 2 \mathrm{C}-\mathrm{C} 11 \mathrm{C}-\mathrm{C} 10 \mathrm{C}$ & $121.1(4)$ \\
\hline $\mathrm{C} 3 \mathrm{~B}-\mathrm{N} 2 \mathrm{~B}-\mathrm{C} 4 \mathrm{~B}$ & $107.8(4)$ & $\mathrm{O} 1 \mathrm{C}-\mathrm{C} 11 \mathrm{C}-\mathrm{C} 10 \mathrm{C}$ & $114.8(4)$ \\
\hline $\mathrm{C} 12 \mathrm{~B}-\mathrm{N} 2 \mathrm{~B}-\mathrm{C} 4 \mathrm{~B}$ & $108.2(4)$ & $\mathrm{N} 2 \mathrm{C}-\mathrm{C} 12 \mathrm{C}-\mathrm{C} 13 \mathrm{C}$ & $111.8(4)$ \\
\hline $\mathrm{C} 3 \mathrm{~B}-\mathrm{N} 2 \mathrm{~B}-\mathrm{Zr} 2$ & $116.7(3)$ & $\mathrm{N} 2 \mathrm{C}-\mathrm{C} 12 \mathrm{C}-\mathrm{H} 12 \mathrm{E}$ & 109.3 \\
\hline C12B-N2B-Zr2 & $106.1(3)$ & $\mathrm{C} 13 \mathrm{C}-\mathrm{C} 12 \mathrm{C}-\mathrm{H} 12 \mathrm{E}$ & 109.3 \\
\hline $\mathrm{C} 4 \mathrm{~B}-\mathrm{N} 2 \mathrm{~B}-\mathrm{Zr} 2$ & $110.1(3)$ & $\mathrm{N} 2 \mathrm{C}-\mathrm{C} 12 \mathrm{C}-\mathrm{H} 12 \mathrm{~F}$ & 109.3 \\
\hline C14B-N3B-C5B & $110.1(4)$ & $\mathrm{C} 13 \mathrm{C}-\mathrm{C} 12 \mathrm{C}-\mathrm{H} 12 \mathrm{~F}$ & 109.3 \\
\hline $\mathrm{C} 14 \mathrm{~B}-\mathrm{N} 3 \mathrm{~B}-\mathrm{C} 6 \mathrm{~B}$ & $108.6(4)$ & $\mathrm{H} 12 \mathrm{E}-\mathrm{C} 12 \mathrm{C}-\mathrm{H} 12 \mathrm{~F}$ & 107.9 \\
\hline C5B-N3B-C6B & $109.0(4)$ & $\mathrm{O} 4 \mathrm{C}-\mathrm{C} 13 \mathrm{C}-\mathrm{O} 3 \mathrm{C}$ & $124.3(4)$ \\
\hline C14B-N3B-Zr2 & $107.3(3)$ & $\mathrm{O} 4 \mathrm{C}-\mathrm{C} 13 \mathrm{C}-\mathrm{C} 12 \mathrm{C}$ & $120.2(4)$ \\
\hline $\mathrm{C} 5 \mathrm{~B}-\mathrm{N} 3 \mathrm{~B}-\mathrm{Zr} 2$ & $109.6(3)$ & $\mathrm{O} 3 \mathrm{C}-\mathrm{C} 13 \mathrm{C}-\mathrm{C} 12 \mathrm{C}$ & $115.5(4)$ \\
\hline C6B-N3B-Zr2 & $112.3(3)$ & N3C-C14C-C15C & $111.8(4)$ \\
\hline
\end{tabular}




\begin{tabular}{|c|c|c|c|}
\hline $\mathrm{C} 8 \mathrm{~B}-\mathrm{N} 4 \mathrm{~B}-\mathrm{C} 16 \mathrm{~B}$ & $109.5(4)$ & $\mathrm{N} 3 \mathrm{C}-\mathrm{C} 14 \mathrm{C}-\mathrm{H} 14 \mathrm{E}$ & 109.3 \\
\hline $\mathrm{C} 8 \mathrm{~B}-\mathrm{N} 4 \mathrm{~B}-\mathrm{C} 7 \mathrm{~B}$ & $107.8(4)$ & C15C-C14C-H14E & 109.3 \\
\hline $\mathrm{C} 16 \mathrm{~B}-\mathrm{N} 4 \mathrm{~B}-\mathrm{C} 7 \mathrm{~B}$ & $108.7(4)$ & $\mathrm{N} 3 \mathrm{C}-\mathrm{C} 14 \mathrm{C}-\mathrm{H} 14 \mathrm{~F}$ & 109.3 \\
\hline $\mathrm{C} 8 \mathrm{~B}-\mathrm{N} 4 \mathrm{~B}-\mathrm{Zr} 2$ & $114.9(3)$ & $\mathrm{C} 15 \mathrm{C}-\mathrm{C} 14 \mathrm{C}-\mathrm{H} 14 \mathrm{~F}$ & 109.3 \\
\hline C16B-N4B-Zr2 & $101.6(3)$ & $\mathrm{H} 14 \mathrm{E}-\mathrm{C} 14 \mathrm{C}-\mathrm{H} 14 \mathrm{~F}$ & 107.9 \\
\hline C7B-N4B-Zr2 & $114.1(3)$ & $\mathrm{O} 6 \mathrm{C}-\mathrm{C} 15 \mathrm{C}-\mathrm{O} 5 \mathrm{C}$ & $123.5(4)$ \\
\hline N1B-C1B-C2B & $115.4(4)$ & $\mathrm{O} 6 \mathrm{C}-\mathrm{C} 15 \mathrm{C}-\mathrm{C} 14 \mathrm{C}$ & $120.8(4)$ \\
\hline $\mathrm{N} 1 \mathrm{~B}-\mathrm{C} 1 \mathrm{~B}-\mathrm{H} 1 \mathrm{C}$ & 108.4 & $\mathrm{O} 5 \mathrm{C}-\mathrm{C} 15 \mathrm{C}-\mathrm{C} 14 \mathrm{C}$ & $115.6(4)$ \\
\hline $\mathrm{C} 2 \mathrm{~B}-\mathrm{C} 1 \mathrm{~B}-\mathrm{H} 1 \mathrm{C}$ & 108.4 & $\mathrm{~N} 4 \mathrm{C}-\mathrm{C} 16 \mathrm{C}-\mathrm{C} 17 \mathrm{C}$ & $113.2(4)$ \\
\hline N1B-C1B-H1D & 108.4 & $\mathrm{~N} 4 \mathrm{C}-\mathrm{C} 16 \mathrm{C}-\mathrm{H} 16 \mathrm{E}$ & 108.9 \\
\hline $\mathrm{C} 2 \mathrm{~B}-\mathrm{C} 1 \mathrm{~B}-\mathrm{H} 1 \mathrm{D}$ & 108.4 & C17C-C16C-H16E & 108.9 \\
\hline $\mathrm{H} 1 \mathrm{C}-\mathrm{C} 1 \mathrm{~B}-\mathrm{H} 1 \mathrm{D}$ & 107.5 & $\mathrm{~N} 4 \mathrm{C}-\mathrm{C} 16 \mathrm{C}-\mathrm{H} 16 \mathrm{~F}$ & 108.9 \\
\hline $\mathrm{C} 3 \mathrm{~B}-\mathrm{C} 2 \mathrm{~B}-\mathrm{C} 1 \mathrm{~B}$ & $115.9(5)$ & C17C-C16C-H16F & 108.9 \\
\hline $\mathrm{C} 3 \mathrm{~B}-\mathrm{C} 2 \mathrm{~B}-\mathrm{H} 2 \mathrm{C}$ & 108.3 & $\mathrm{H} 16 \mathrm{E}-\mathrm{C} 16 \mathrm{C}-\mathrm{H} 16 \mathrm{~F}$ & 107.8 \\
\hline $\mathrm{C} 1 \mathrm{~B}-\mathrm{C} 2 \mathrm{~B}-\mathrm{H} 2 \mathrm{C}$ & 108.3 & $\mathrm{O} 8 \mathrm{C}-\mathrm{C} 17 \mathrm{C}-\mathrm{O} 7 \mathrm{C}$ & $125.3(5)$ \\
\hline C3B-C2B-H2D & 108.3 & $\mathrm{O} 8 \mathrm{C}-\mathrm{C} 17 \mathrm{C}-\mathrm{C} 16 \mathrm{C}$ & $118.3(4)$ \\
\hline C1B-C2B-H2D & 108.3 & O7C-C17C-C16C & $116.4(4)$ \\
\hline $\mathrm{H} 2 \mathrm{C}-\mathrm{C} 2 \mathrm{~B}-\mathrm{H} 2 \mathrm{D}$ & 107.4 & $\mathrm{H} 11-\mathrm{O} 1-\mathrm{H} 12$ & $104(3)$ \\
\hline $\mathrm{N} 2 \mathrm{~B}-\mathrm{C} 3 \mathrm{~B}-\mathrm{C} 2 \mathrm{~B}$ & $116.0(4)$ & $\mathrm{H} 21-\mathrm{O} 2-\mathrm{H} 22$ & $103(3)$ \\
\hline $\mathrm{N} 2 \mathrm{~B}-\mathrm{C} 3 \mathrm{~B}-\mathrm{H} 3 \mathrm{C}$ & 108.3 & $\mathrm{H} 31-\mathrm{O} 3-\mathrm{H} 32$ & $108(3)$ \\
\hline $\mathrm{C} 2 \mathrm{~B}-\mathrm{C} 3 \mathrm{~B}-\mathrm{H} 3 \mathrm{C}$ & 108.3 & $\mathrm{H} 41-\mathrm{O} 4-\mathrm{H} 42$ & $104(3)$ \\
\hline $\mathrm{N} 2 \mathrm{~B}-\mathrm{C} 3 \mathrm{~B}-\mathrm{H} 3 \mathrm{D}$ & 108.3 & H51-O5-H52 & $107(4)$ \\
\hline $\mathrm{C} 2 \mathrm{~B}-\mathrm{C} 3 \mathrm{~B}-\mathrm{H} 3 \mathrm{D}$ & 108.3 & $\mathrm{H} 61-\mathrm{O} 6-\mathrm{H} 62$ & $107(3)$ \\
\hline $\mathrm{H} 3 \mathrm{C}-\mathrm{C} 3 \mathrm{~B}-\mathrm{H} 3 \mathrm{D}$ & 107.4 & $\mathrm{H} 71-\mathrm{O} 7-\mathrm{H} 72$ & $106(3)$ \\
\hline $\mathrm{C} 5 \mathrm{~B}-\mathrm{C} 4 \mathrm{~B}-\mathrm{N} 2 \mathrm{~B}$ & $111.2(4)$ & H81-O8-H82 & $107(4)$ \\
\hline C5B-C4B-H4C & 109.4 & $\mathrm{H} 91-\mathrm{O} 9-\mathrm{H} 92$ & $102(3)$ \\
\hline $\mathrm{N} 2 \mathrm{~B}-\mathrm{C} 4 \mathrm{~B}-\mathrm{H} 4 \mathrm{C}$ & 109.4 & $\mathrm{H} 101-\mathrm{O} 10-\mathrm{H} 102$ & $107(3)$ \\
\hline C5B-C4B-H4D & 109.4 & C1B-N1B-C10B-C11B & $-169.4(4)$ \\
\hline $\mathrm{C} 10 \mathrm{~A}-\mathrm{N} 1 \mathrm{~A}-\mathrm{C} 1 \mathrm{~A}-\mathrm{C} 2 \mathrm{~A}$ & $89.1(5)$ & C9B-N1B-C10B-C11B & $75.6(4)$ \\
\hline
\end{tabular}




\begin{tabular}{|c|c|c|c|}
\hline $\mathrm{C} 9 \mathrm{~A}-\mathrm{N} 1 \mathrm{~A}-\mathrm{C} 1 \mathrm{~A}-\mathrm{C} 2 \mathrm{~A}$ & $-157.1(4)$ & $\mathrm{Zr} 2-\mathrm{N} 1 \mathrm{~B}-\mathrm{C} 10 \mathrm{~B}-\mathrm{C} 11 \mathrm{~B}$ & $-37.7(4)$ \\
\hline $\mathrm{Zr} 1-\mathrm{N} 1 \mathrm{~A}-\mathrm{C} 1 \mathrm{~A}-\mathrm{C} 2 \mathrm{~A}$ & $-31.8(5)$ & $\mathrm{Zr} 2-\mathrm{O} 1 \mathrm{~B}-\mathrm{C} 11 \mathrm{~B}-\mathrm{O} 2 \mathrm{~B}$ & $-155.5(4)$ \\
\hline $\mathrm{N} 1 \mathrm{~A}-\mathrm{C} 1 \mathrm{~A}-\mathrm{C} 2 \mathrm{~A}-\mathrm{C} 3 \mathrm{~A}$ & $69.3(5)$ & $\mathrm{Zr} 2-\mathrm{O} 1 \mathrm{~B}-\mathrm{C} 11 \mathrm{~B}-\mathrm{C} 10 \mathrm{~B}$ & $25.6(5)$ \\
\hline $\mathrm{C} 1 \mathrm{~A}-\mathrm{C} 2 \mathrm{~A}-\mathrm{C} 3 \mathrm{~A}-\mathrm{N} 2 \mathrm{~A}$ & $-89.6(5)$ & $\mathrm{N} 1 \mathrm{~B}-\mathrm{C} 10 \mathrm{~B}-\mathrm{C} 11 \mathrm{~B}-\mathrm{O} 2 \mathrm{~B}$ & $-163.7(4)$ \\
\hline $\mathrm{C} 12 \mathrm{~A}-\mathrm{N} 2 \mathrm{~A}-\mathrm{C} 3 \mathrm{~A}-\mathrm{C} 2 \mathrm{~A}$ & $-61.1(5)$ & $\mathrm{N} 1 \mathrm{~B}-\mathrm{C} 10 \mathrm{~B}-\mathrm{C} 11 \mathrm{~B}-\mathrm{O} 1 \mathrm{~B}$ & $15.3(6)$ \\
\hline $\mathrm{C} 4 \mathrm{~A}-\mathrm{N} 2 \mathrm{~A}-\mathrm{C} 3 \mathrm{~A}-\mathrm{C} 2 \mathrm{~A}$ & $-177.6(4)$ & C3B-N2B-C12B-C13B & $96.4(5)$ \\
\hline $\mathrm{Zr} 1-\mathrm{N} 2 \mathrm{~A}-\mathrm{C} 3 \mathrm{~A}-\mathrm{C} 2 \mathrm{~A}$ & $58.6(4)$ & $\mathrm{C} 4 \mathrm{~B}-\mathrm{N} 2 \mathrm{~B}-\mathrm{C} 12 \mathrm{~B}-\mathrm{C} 13 \mathrm{~B}$ & $-147.4(4)$ \\
\hline C12A-N2A-C4A-C5A & $159.1(4)$ & $\mathrm{Zr} 2-\mathrm{N} 2 \mathrm{~B}-\mathrm{C} 12 \mathrm{~B}-\mathrm{C} 13 \mathrm{~B}$ & $-29.3(5)$ \\
\hline C3A-N2A-C4A-C5A & $-84.6(4)$ & $\mathrm{Zr} 2-\mathrm{O} 3 \mathrm{~B}-\mathrm{C} 13 \mathrm{~B}-\mathrm{O} 4 \mathrm{~B}$ & $-169.8(4)$ \\
\hline $\mathrm{Zr} 1-\mathrm{N} 2 \mathrm{~A}-\mathrm{C} 4 \mathrm{~A}-\mathrm{C} 5 \mathrm{~A}$ & $41.4(4)$ & $\mathrm{Zr} 2-\mathrm{O} 3 \mathrm{~B}-\mathrm{C} 13 \mathrm{~B}-\mathrm{C} 12 \mathrm{~B}$ & $8.4(6)$ \\
\hline $\mathrm{C} 14 \mathrm{~A}-\mathrm{N} 3 \mathrm{~A}-\mathrm{C} 5 \mathrm{~A}-\mathrm{C} 4 \mathrm{~A}$ & $-73.3(4)$ & $\mathrm{N} 2 \mathrm{~B}-\mathrm{C} 12 \mathrm{~B}-\mathrm{C} 13 \mathrm{~B}-\mathrm{O} 4 \mathrm{~B}$ & $-164.2(5)$ \\
\hline $\mathrm{C} 6 \mathrm{~A}-\mathrm{N} 3 \mathrm{~A}-\mathrm{C} 5 \mathrm{~A}-\mathrm{C} 4 \mathrm{~A}$ & $167.2(4)$ & $\mathrm{N} 2 \mathrm{~B}-\mathrm{C} 12 \mathrm{~B}-\mathrm{C} 13 \mathrm{~B}-\mathrm{O} 3 \mathrm{~B}$ & $17.5(6)$ \\
\hline $\mathrm{Zr} 1-\mathrm{N} 3 \mathrm{~A}-\mathrm{C} 5 \mathrm{~A}-\mathrm{C} 4 \mathrm{~A}$ & 43.7 (4) & C5B-N3B-C14B-C15B & $94.9(5)$ \\
\hline $\mathrm{N} 2 \mathrm{~A}-\mathrm{C} 4 \mathrm{~A}-\mathrm{C} 5 \mathrm{~A}-\mathrm{N} 3 \mathrm{~A}$ & $-58.2(5)$ & $\mathrm{C} 6 \mathrm{~B}-\mathrm{N} 3 \mathrm{~B}-\mathrm{C} 14 \mathrm{~B}-\mathrm{C} 15 \mathrm{~B}$ & $-145.8(4)$ \\
\hline $\mathrm{C} 14 \mathrm{~A}-\mathrm{N} 3 \mathrm{~A}-\mathrm{C} 6 \mathrm{~A}-\mathrm{C} 7 \mathrm{~A}$ & $163.7(4)$ & $\mathrm{Zr} 2-\mathrm{N} 3 \mathrm{~B}-\mathrm{C} 14 \mathrm{~B}-\mathrm{C} 15 \mathrm{~B}$ & $-24.3(5)$ \\
\hline$C 5 A-N 3 A-C 6 A-C 7 A$ & $-77.0(4)$ & $\mathrm{Zr} 2-\mathrm{O}$ BB-C15B-O6B & $-168.4(3)$ \\
\hline $\mathrm{Zr} 1-\mathrm{N} 3 \mathrm{~A}-\mathrm{C} 6 \mathrm{~A}-\mathrm{C} 7 \mathrm{~A}$ & $45.0(4)$ & Zr2-O5B-C15B-C14B & $9.6(6)$ \\
\hline $\mathrm{N} 3 \mathrm{~A}-\mathrm{C} 6 \mathrm{~A}-\mathrm{C} 7 \mathrm{~A}-\mathrm{N} 4 \mathrm{~A}$ & $-55.3(5)$ & N3B-C14B-C15B-O6B & $-168.7(4)$ \\
\hline C16A-N4A-C7A-C6A & $-76.8(4)$ & N3B $-C 14 B-C 15 B-O 5 B$ & $13.3(6)$ \\
\hline $\mathrm{C} 8 \mathrm{~A}-\mathrm{N} 4 \mathrm{~A}-\mathrm{C} 7 \mathrm{~A}-\mathrm{C} 6 \mathrm{~A}$ & $165.1(4)$ & $\mathrm{C} 8 \mathrm{~B}-\mathrm{N} 4 \mathrm{~B}-\mathrm{C} 16 \mathrm{~B}-\mathrm{C} 17 \mathrm{~B}$ & $-161.0(4)$ \\
\hline $\mathrm{Zr} 1-\mathrm{N} 4 \mathrm{~A}-\mathrm{C} 7 \mathrm{~A}-\mathrm{C} 6 \mathrm{~A}$ & $38.6(4)$ & C7B-N4B-C16B-C17B & $81.5(5)$ \\
\hline C16A-N4A-C8A-C9A & $78.2(4)$ & $\mathrm{Zr} 2-\mathrm{N} 4 \mathrm{~B}-\mathrm{C} 16 \mathrm{~B}-\mathrm{C} 17 \mathrm{~B}$ & $-39.1(4)$ \\
\hline $\mathrm{C} 7 \mathrm{~A}-\mathrm{N} 4 \mathrm{~A}-\mathrm{C} 8 \mathrm{~A}-\mathrm{C} 9 \mathrm{~A}$ & $-163.3(3)$ & $\mathrm{Zr} 2-\mathrm{O} 7 \mathrm{~B}-\mathrm{C} 17 \mathrm{~B}-\mathrm{O} 8 \mathrm{~B}$ & $-173.4(4)$ \\
\hline $\mathrm{Zr} 1-\mathrm{N} 4 \mathrm{~A}-\mathrm{C} 8 \mathrm{~A}-\mathrm{C} 9 \mathrm{~A}$ & $-37.2(4)$ & Zr2-O7B-C17B-C16B & $6.9(6)$ \\
\hline $\mathrm{N} 4 \mathrm{~A}-\mathrm{C} 8 \mathrm{~A}-\mathrm{C} 9 \mathrm{~A}-\mathrm{N} 1 \mathrm{~A}$ & $60.7(4)$ & $\mathrm{N} 4 \mathrm{~B}-\mathrm{C} 16 \mathrm{~B}-\mathrm{C} 17 \mathrm{~B}-\mathrm{O} 8 \mathrm{~B}$ & $-153.1(5)$ \\
\hline $\mathrm{C} 1 \mathrm{~A}-\mathrm{N} 1 \mathrm{~A}-\mathrm{C} 9 \mathrm{~A}-\mathrm{C} 8 \mathrm{~A}$ & $82.7(4)$ & N4B-C16B-C17B-O7B & $26.6(6)$ \\
\hline $\mathrm{C} 10 \mathrm{~A}-\mathrm{N} 1 \mathrm{~A}-\mathrm{C} 9 \mathrm{~A}-\mathrm{C} 8 \mathrm{~A}$ & $-161.3(3)$ & $\mathrm{C} 9 \mathrm{C}-\mathrm{N} 1 \mathrm{C}-\mathrm{C} 1 \mathrm{C}-\mathrm{C} 2 \mathrm{C}$ & $-157.5(4)$ \\
\hline $\mathrm{Zr} 1-\mathrm{N} 1 \mathrm{~A}-\mathrm{C} 9 \mathrm{~A}-\mathrm{C} 8 \mathrm{~A}$ & $-51.9(4)$ & $\mathrm{C} 10 \mathrm{C}-\mathrm{N} 1 \mathrm{C}-\mathrm{C} 1 \mathrm{C}-\mathrm{C} 2 \mathrm{C}$ & $88.7(5)$ \\
\hline $\mathrm{C} 1 \mathrm{~A}-\mathrm{N} 1 \mathrm{~A}-\mathrm{C} 10 \mathrm{~A}-\mathrm{C} 11 \mathrm{~A}$ & $-167.9(3)$ & $\mathrm{Zr} 3-\mathrm{N} 1 \mathrm{C}-\mathrm{C} 1 \mathrm{C}-\mathrm{C} 2 \mathrm{C}$ & $-31.6(5)$ \\
\hline
\end{tabular}




\begin{tabular}{|c|c|c|c|}
\hline $\mathrm{C} 9 \mathrm{~A}-\mathrm{N} 1 \mathrm{~A}-\mathrm{C} 10 \mathrm{~A}-\mathrm{C} 11 \mathrm{~A}$ & $77.3(4)$ & $\mathrm{N} 1 \mathrm{C}-\mathrm{C} 1 \mathrm{C}-\mathrm{C} 2 \mathrm{C}-\mathrm{C} 3 \mathrm{C}$ & $69.5(5)$ \\
\hline $\mathrm{Zr} 1-\mathrm{N} 1 \mathrm{~A}-\mathrm{C} 10 \mathrm{~A}-\mathrm{C} 11 \mathrm{~A}$ & $-35.0(4)$ & $\mathrm{C} 4 \mathrm{C}-\mathrm{N} 2 \mathrm{C}-\mathrm{C} 3 \mathrm{C}-\mathrm{C} 2 \mathrm{C}$ & $-177.5(4)$ \\
\hline $\mathrm{Zr} 1-\mathrm{O} 1 \mathrm{~A}-\mathrm{C} 11 \mathrm{~A}-\mathrm{O} 2 \mathrm{~A}$ & $-149.9(4)$ & $\mathrm{C} 12 \mathrm{C}-\mathrm{N} 2 \mathrm{C}-\mathrm{C} 3 \mathrm{C}-\mathrm{C} 2 \mathrm{C}$ & $-60.9(5)$ \\
\hline $\mathrm{Zr} 1-\mathrm{O} 1 \mathrm{~A}-\mathrm{C} 11 \mathrm{~A}-\mathrm{C} 10 \mathrm{~A}$ & $30.5(5)$ & $\mathrm{Zr} 3-\mathrm{N} 2 \mathrm{C}-\mathrm{C} 3 \mathrm{C}-\mathrm{C} 2 \mathrm{C}$ & $58.6(4)$ \\
\hline $\mathrm{N} 1 \mathrm{~A}-\mathrm{C} 10 \mathrm{~A}-\mathrm{C} 11 \mathrm{~A}-\mathrm{O} 2 \mathrm{~A}$ & $-168.7(4)$ & $\mathrm{C} 1 \mathrm{C}-\mathrm{C} 2 \mathrm{C}-\mathrm{C} 3 \mathrm{C}-\mathrm{N} 2 \mathrm{C}$ & $-89.9(5)$ \\
\hline $\mathrm{N} 1 \mathrm{~A}-\mathrm{C} 10 \mathrm{~A}-\mathrm{C} 11 \mathrm{~A}-\mathrm{O} 1 \mathrm{~A}$ & $10.8(5)$ & $\mathrm{C} 12 \mathrm{C}-\mathrm{N} 2 \mathrm{C}-\mathrm{C} 4 \mathrm{C}-\mathrm{C} 5 \mathrm{C}$ & $158.6(4)$ \\
\hline $\mathrm{C} 4 \mathrm{~A}-\mathrm{N} 2 \mathrm{~A}-\mathrm{C} 12 \mathrm{~A}-\mathrm{C} 13 \mathrm{~A}$ & $-149.8(4)$ & $\mathrm{C} 3 \mathrm{C}-\mathrm{N} 2 \mathrm{C}-\mathrm{C} 4 \mathrm{C}-\mathrm{C} 5 \mathrm{C}$ & $-84.3(4)$ \\
\hline C3A-N2A-C12A-C13A & $93.7(4)$ & $\mathrm{Zr} 3-\mathrm{N} 2 \mathrm{C}-\mathrm{C} 4 \mathrm{C}-\mathrm{C} 5 \mathrm{C}$ & $42.0(4)$ \\
\hline $\mathrm{Zr} 1-\mathrm{N} 2 \mathrm{~A}-\mathrm{C} 12 \mathrm{~A}-\mathrm{C} 13 \mathrm{~A}$ & $-30.2(4)$ & $\mathrm{C} 14 \mathrm{C}-\mathrm{N} 3 \mathrm{C}-\mathrm{C} 5 \mathrm{C}-\mathrm{C} 4 \mathrm{C}$ & $-73.8(4)$ \\
\hline $\mathrm{Zr} 1-\mathrm{O} 3 \mathrm{~A}-\mathrm{C} 13 \mathrm{~A}-\mathrm{O} 4 \mathrm{~A}$ & $-178.0(3)$ & $\mathrm{C} 6 \mathrm{C}-\mathrm{N} 3 \mathrm{C}-\mathrm{C} 5 \mathrm{C}-\mathrm{C} 4 \mathrm{C}$ & $167.3(4)$ \\
\hline $\mathrm{Zr} 1-\mathrm{O} 3 \mathrm{~A}-\mathrm{C} 13 \mathrm{~A}-\mathrm{C} 12 \mathrm{~A}$ & $0.8(5)$ & $\mathrm{Zr} 3-\mathrm{N} 3 \mathrm{C}-\mathrm{C} 5 \mathrm{C}-\mathrm{C} 4 \mathrm{C}$ & $43.7(4)$ \\
\hline $\mathrm{N} 2 \mathrm{~A}-\mathrm{C} 12 \mathrm{~A}-\mathrm{C} 13 \mathrm{~A}-\mathrm{O} 4 \mathrm{~A}$ & $-158.9(4)$ & $\mathrm{N} 2 \mathrm{C}-\mathrm{C} 4 \mathrm{C}-\mathrm{C} 5 \mathrm{C}-\mathrm{N} 3 \mathrm{C}$ & $-58.6(5)$ \\
\hline $\mathrm{N} 2 \mathrm{~A}-\mathrm{C} 12 \mathrm{~A}-\mathrm{C} 13 \mathrm{~A}-\mathrm{O} 3 \mathrm{~A}$ & $22.2(6)$ & $\mathrm{C} 14 \mathrm{C}-\mathrm{N} 3 \mathrm{C}-\mathrm{C} 6 \mathrm{C}-\mathrm{C} 7 \mathrm{C}$ & $163.9(4)$ \\
\hline $\mathrm{C} 6 \mathrm{~A}-\mathrm{N} 3 \mathrm{~A}-\mathrm{C} 14 \mathrm{~A}-\mathrm{C} 15 \mathrm{~A}$ & $-152.5(4)$ & $\mathrm{C} 5 \mathrm{C}-\mathrm{N} 3 \mathrm{C}-\mathrm{C} 6 \mathrm{C}-\mathrm{C} 7 \mathrm{C}$ & $-77.0(4)$ \\
\hline $\mathrm{C} 5 \mathrm{~A}-\mathrm{N} 3 \mathrm{~A}-\mathrm{C} 14 \mathrm{~A}-\mathrm{C} 15 \mathrm{~A}$ & $88.9(4)$ & $\mathrm{Zr} 3-\mathrm{N} 3 \mathrm{C}-\mathrm{C} 6 \mathrm{C}-\mathrm{C} 7 \mathrm{C}$ & $45.1(4)$ \\
\hline $\mathrm{Zr} 1-\mathrm{N} 3 \mathrm{~A}-\mathrm{C} 14 \mathrm{~A}-\mathrm{C} 15 \mathrm{~A}$ & $-30.2(4)$ & $\mathrm{C} 8 \mathrm{C}-\mathrm{N} 4 \mathrm{C}-\mathrm{C} 7 \mathrm{C}-\mathrm{C} 6 \mathrm{C}$ & $165.2(4)$ \\
\hline $\mathrm{Zr} 1-\mathrm{O} \mathrm{AA}-\mathrm{C} 15 \mathrm{~A}-\mathrm{O} \mathrm{6A}$ & $178.4(3)$ & $\mathrm{C} 16 \mathrm{C}-\mathrm{N} 4 \mathrm{C}-\mathrm{C} 7 \mathrm{C}-\mathrm{C} 6 \mathrm{C}$ & $-76.7(4)$ \\
\hline $\mathrm{Zr} 1-\mathrm{O} 5 \mathrm{~A}-\mathrm{C} 15 \mathrm{~A}-\mathrm{C} 14 \mathrm{~A}$ & $-2.9(5)$ & $\mathrm{Zr} 3-\mathrm{N} 4 \mathrm{C}-\mathrm{C} 7 \mathrm{C}-\mathrm{C} 6 \mathrm{C}$ & $38.4(4)$ \\
\hline $\mathrm{N} 3 \mathrm{~A}-\mathrm{C} 14 \mathrm{~A}-\mathrm{C} 15 \mathrm{~A}-\mathrm{O} 6 \mathrm{~A}$ & $-156.6(4)$ & $\mathrm{N} 3 \mathrm{C}-\mathrm{C} 6 \mathrm{C}-\mathrm{C} 7 \mathrm{C}-\mathrm{N} 4 \mathrm{C}$ & $-55.3(5)$ \\
\hline N3A-C14A-C15A-O5A & $24.6(5)$ & $\mathrm{C} 7 \mathrm{C}-\mathrm{N} 4 \mathrm{C}-\mathrm{C} 8 \mathrm{C}-\mathrm{C} 9 \mathrm{C}$ & $-163.1(4)$ \\
\hline C7A-N4A-C16A-C17A & $91.3(5)$ & $\mathrm{C} 16 \mathrm{C}-\mathrm{N} 4 \mathrm{C}-\mathrm{C} 8 \mathrm{C}-\mathrm{C} 9 \mathrm{C}$ & $78.8(4)$ \\
\hline $\mathrm{C} 8 \mathrm{~A}-\mathrm{N} 4 \mathrm{~A}-\mathrm{C} 16 \mathrm{~A}-\mathrm{C} 17 \mathrm{~A}$ & $-151.4(4)$ & $\mathrm{Zr} 3-\mathrm{N} 4 \mathrm{C}-\mathrm{C} 8 \mathrm{C}-\mathrm{C} 9 \mathrm{C}$ & $-36.6(4)$ \\
\hline $\mathrm{Zr} 1-\mathrm{N} 4 \mathrm{~A}-\mathrm{C} 16 \mathrm{~A}-\mathrm{C} 17 \mathrm{~A}$ & $-29.9(4)$ & $\mathrm{C} 10 \mathrm{C}-\mathrm{N} 1 \mathrm{C}-\mathrm{C} 9 \mathrm{C}-\mathrm{C} 8 \mathrm{C}$ & $-160.7(4)$ \\
\hline $\mathrm{Zr} 1-\mathrm{O} 7 \mathrm{~A}-\mathrm{C} 17 \mathrm{~A}-\mathrm{O} 8 \mathrm{~A}$ & $-157.8(5)$ & $\mathrm{C} 1 \mathrm{C}-\mathrm{N} 1 \mathrm{C}-\mathrm{C} 9 \mathrm{C}-\mathrm{C} 8 \mathrm{C}$ & $83.3(4)$ \\
\hline $\mathrm{Zr} 1-\mathrm{O} 7 \mathrm{~A}-\mathrm{C} 17 \mathrm{~A}-\mathrm{C} 16 \mathrm{~A}$ & $22.2(6)$ & $\mathrm{Zr} 3-\mathrm{N} 1 \mathrm{C}-\mathrm{C} 9 \mathrm{C}-\mathrm{C} 8 \mathrm{C}$ & $-51.7(4)$ \\
\hline $\mathrm{N} 4 \mathrm{~A}-\mathrm{C} 16 \mathrm{~A}-\mathrm{C} 17 \mathrm{~A}-\mathrm{O} 8 \mathrm{~A}$ & $-169.9(5)$ & $\mathrm{N} 4 \mathrm{C}-\mathrm{C} 8 \mathrm{C}-\mathrm{C} 9 \mathrm{C}-\mathrm{N} 1 \mathrm{C}$ & $60.2(4)$ \\
\hline N4A-C16A-C17A-O7A & $10.1(6)$ & $\mathrm{C} 9 \mathrm{C}-\mathrm{N} 1 \mathrm{C}-\mathrm{C} 10 \mathrm{C}-\mathrm{C} 11 \mathrm{C}$ & $76.8(4)$ \\
\hline $\mathrm{C} 10 \mathrm{~B}-\mathrm{N} 1 \mathrm{~B}-\mathrm{C} 1 \mathrm{~B}-\mathrm{C} 2 \mathrm{~B}$ & $87.9(5)$ & $\mathrm{C} 1 \mathrm{C}-\mathrm{N} 1 \mathrm{C}-\mathrm{C} 10 \mathrm{C}-\mathrm{C} 11 \mathrm{C}$ & $-168.2(3)$ \\
\hline $\mathrm{C} 9 \mathrm{~B}-\mathrm{N} 1 \mathrm{~B}-\mathrm{C} 1 \mathrm{~B}-\mathrm{C} 2 \mathrm{~B}$ & $-158.2(5)$ & $\mathrm{Zr} 3-\mathrm{N} 1 \mathrm{C}-\mathrm{C} 10 \mathrm{C}-\mathrm{C} 11 \mathrm{C}$ & $-35.8(4)$ \\
\hline
\end{tabular}




\begin{tabular}{|c|c|c|c|}
\hline $\mathrm{Zr} 2-\mathrm{N} 1 \mathrm{~B}-\mathrm{C} 1 \mathrm{~B}-\mathrm{C} 2 \mathrm{~B}$ & $-33.3(6)$ & $\mathrm{Zr} 3-\mathrm{O} 1 \mathrm{C}-\mathrm{C} 11 \mathrm{C}-\mathrm{O} 2 \mathrm{C}$ & $-150.0(4)$ \\
\hline $\mathrm{N} 1 \mathrm{~B}-\mathrm{C} 1 \mathrm{~B}-\mathrm{C} 2 \mathrm{~B}-\mathrm{C} 3 \mathrm{~B}$ & $71.5(6)$ & $\mathrm{Zr} 3-\mathrm{O} 1 \mathrm{C}-\mathrm{C} 11 \mathrm{C}-\mathrm{C} 10 \mathrm{C}$ & $29.8(5)$ \\
\hline $\mathrm{C} 12 \mathrm{~B}-\mathrm{N} 2 \mathrm{~B}-\mathrm{C} 3 \mathrm{~B}-\mathrm{C} 2 \mathrm{~B}$ & $-65.6(5)$ & $\mathrm{N} 1 \mathrm{C}-\mathrm{C} 10 \mathrm{C}-\mathrm{C} 11 \mathrm{C}-\mathrm{O} 2 \mathrm{C}$ & $-168.3(4)$ \\
\hline $\mathrm{C} 4 \mathrm{~B}-\mathrm{N} 2 \mathrm{~B}-\mathrm{C} 3 \mathrm{~B}-\mathrm{C} 2 \mathrm{~B}$ & $177.9(4)$ & $\mathrm{N} 1 \mathrm{C}-\mathrm{C} 10 \mathrm{C}-\mathrm{C} 11 \mathrm{C}-\mathrm{O} 1 \mathrm{C}$ & $11.9(5)$ \\
\hline $\mathrm{Zr} 2-\mathrm{N} 2 \mathrm{~B}-\mathrm{C} 3 \mathrm{~B}-\mathrm{C} 2 \mathrm{~B}$ & $53.4(5)$ & $\mathrm{C} 4 \mathrm{C}-\mathrm{N} 2 \mathrm{C}-\mathrm{C} 12 \mathrm{C}-\mathrm{C} 13 \mathrm{C}$ & $-150.0(4)$ \\
\hline $\mathrm{C} 1 \mathrm{~B}-\mathrm{C} 2 \mathrm{~B}-\mathrm{C} 3 \mathrm{~B}-\mathrm{N} 2 \mathrm{~B}$ & $-86.2(6)$ & $\mathrm{C} 3 \mathrm{C}-\mathrm{N} 2 \mathrm{C}-\mathrm{C} 12 \mathrm{C}-\mathrm{C} 13 \mathrm{C}$ & $93.3(4)$ \\
\hline $\mathrm{C} 3 \mathrm{~B}-\mathrm{N} 2 \mathrm{~B}-\mathrm{C} 4 \mathrm{~B}-\mathrm{C} 5 \mathrm{~B}$ & $-86.3(5)$ & $\mathrm{Zr} 3-\mathrm{N} 2 \mathrm{C}-\mathrm{C} 12 \mathrm{C}-\mathrm{C} 13 \mathrm{C}$ & $-30.9(4)$ \\
\hline $\mathrm{C} 12 \mathrm{~B}-\mathrm{N} 2 \mathrm{~B}-\mathrm{C} 4 \mathrm{~B}-\mathrm{C} 5 \mathrm{~B}$ & $157.5(4)$ & $\mathrm{Zr} 3-\mathrm{O} 3 \mathrm{C}-\mathrm{C} 13 \mathrm{C}-\mathrm{O} 4 \mathrm{C}$ & $-178.8(3)$ \\
\hline $\mathrm{Zr} 2-\mathrm{N} 2 \mathrm{~B}-\mathrm{C} 4 \mathrm{~B}-\mathrm{C} 5 \mathrm{~B}$ & $42.0(5)$ & $\mathrm{Zr} 3-\mathrm{O} 3 \mathrm{C}-\mathrm{C} 13 \mathrm{C}-\mathrm{C} 12 \mathrm{C}$ & $-0.5(5)$ \\
\hline $\mathrm{N} 2 \mathrm{~B}-\mathrm{C} 4 \mathrm{~B}-\mathrm{C} 5 \mathrm{~B}-\mathrm{N} 3 \mathrm{~B}$ & $-60.0(5)$ & $\mathrm{N} 2 \mathrm{C}-\mathrm{C} 12 \mathrm{C}-\mathrm{C} 13 \mathrm{C}-\mathrm{O} 4 \mathrm{C}$ & $-158.0(4)$ \\
\hline $\mathrm{C} 14 \mathrm{~B}-\mathrm{N} 3 \mathrm{~B}-\mathrm{C} 5 \mathrm{~B}-\mathrm{C} 4 \mathrm{~B}$ & $-72.3(5)$ & $\mathrm{N} 2 \mathrm{C}-\mathrm{C} 12 \mathrm{C}-\mathrm{C} 13 \mathrm{C}-\mathrm{O} 3 \mathrm{C}$ & $23.7(6)$ \\
\hline $\mathrm{C} 6 \mathrm{~B}-\mathrm{N} 3 \mathrm{~B}-\mathrm{C} 5 \mathrm{~B}-\mathrm{C} 4 \mathrm{~B}$ & $168.7(4)$ & $\mathrm{C} 6 \mathrm{C}-\mathrm{N} 3 \mathrm{C}-\mathrm{C} 14 \mathrm{C}-\mathrm{C} 15 \mathrm{C}$ & $-151.4(4)$ \\
\hline $\mathrm{Zr} 2-\mathrm{N} 3 \mathrm{~B}-\mathrm{C} 5 \mathrm{~B}-\mathrm{C} 4 \mathrm{~B}$ & $45.5(4)$ & $\mathrm{C} 5 \mathrm{C}-\mathrm{N} 3 \mathrm{C}-\mathrm{C} 14 \mathrm{C}-\mathrm{C} 15 \mathrm{C}$ & $89.8(4)$ \\
\hline $\mathrm{C} 14 \mathrm{~B}-\mathrm{N} 3 \mathrm{~B}-\mathrm{C} 6 \mathrm{~B}-\mathrm{C} 7 \mathrm{~B}$ & $162.2(4)$ & $\mathrm{Zr} 3-\mathrm{N} 3 \mathrm{C}-\mathrm{C} 14 \mathrm{C}-\mathrm{C} 15 \mathrm{C}$ & $-29.5(4)$ \\
\hline $\mathrm{C} 5 \mathrm{~B}-\mathrm{N} 3 \mathrm{~B}-\mathrm{C} 6 \mathrm{~B}-\mathrm{C} 7 \mathrm{~B}$ & $-77.8(5)$ & $\mathrm{Zr} 3-\mathrm{O} 5 \mathrm{C}-\mathrm{C} 15 \mathrm{C}-\mathrm{O} \mathrm{C}$ & $-180.0(3)$ \\
\hline $\mathrm{Zr} 2-\mathrm{N} 3 \mathrm{~B}-\mathrm{C} 6 \mathrm{~B}-\mathrm{C} 7 \mathrm{~B}$ & $43.8(5)$ & $\mathrm{Zr} 3-\mathrm{O} 5 \mathrm{C}-\mathrm{C} 15 \mathrm{C}-\mathrm{C} 14 \mathrm{C}$ & $-1.5(5)$ \\
\hline $\mathrm{N} 3 \mathrm{~B}-\mathrm{C} 6 \mathrm{~B}-\mathrm{C} 7 \mathrm{~B}-\mathrm{N} 4 \mathrm{~B}$ & $-51.7(6)$ & $\mathrm{N} 3 \mathrm{C}-\mathrm{C} 14 \mathrm{C}-\mathrm{C} 15 \mathrm{C}-\mathrm{O} 6 \mathrm{C}$ & $-158.3(4)$ \\
\hline $\mathrm{C} 8 \mathrm{~B}-\mathrm{N} 4 \mathrm{~B}-\mathrm{C} 7 \mathrm{~B}-\mathrm{C} 6 \mathrm{~B}$ & $163.3(4)$ & $\mathrm{N} 3 \mathrm{C}-\mathrm{C} 14 \mathrm{C}-\mathrm{C} 15 \mathrm{C}-\mathrm{O} 5 \mathrm{C}$ & $23.2(5)$ \\
\hline $\mathrm{C} 16 \mathrm{~B}-\mathrm{N} 4 \mathrm{~B}-\mathrm{C} 7 \mathrm{~B}-\mathrm{C} 6 \mathrm{~B}$ & $-78.2(5)$ & $\mathrm{C} 8 \mathrm{C}-\mathrm{N} 4 \mathrm{C}-\mathrm{C} 16 \mathrm{C}-\mathrm{C} 17 \mathrm{C}$ & $-152.5(4)$ \\
\hline Zr2-N4B-C7B-C6B & $34.4(5)$ & C7C-N4C-C16C-C17C & $90.5(4)$ \\
\hline $\mathrm{C} 16 \mathrm{~B}-\mathrm{N} 4 \mathrm{~B}-\mathrm{C} 8 \mathrm{~B}-\mathrm{C} 9 \mathrm{~B}$ & $78.1(5)$ & $\mathrm{Zr} 3-\mathrm{N} 4 \mathrm{C}-\mathrm{C} 16 \mathrm{C}-\mathrm{C} 17 \mathrm{C}$ & $-30.7(4)$ \\
\hline C7B-N4B-C8B-C9B & $-163.8(4)$ & $\mathrm{Zr} 3-\mathrm{O} 7 \mathrm{C}-\mathrm{C} 17 \mathrm{C}-\mathrm{O} 8 \mathrm{C}$ & $-159.1(4)$ \\
\hline $\mathrm{Zr} 2-\mathrm{N} 4 \mathrm{~B}-\mathrm{C} 8 \mathrm{~B}-\mathrm{C} 9 \mathrm{~B}$ & $-35.4(5)$ & $\mathrm{Zr} 3-\mathrm{O} 7 \mathrm{C}-\mathrm{C} 17 \mathrm{C}-\mathrm{C} 16 \mathrm{C}$ & $20.2(5)$ \\
\hline $\mathrm{N} 4 \mathrm{~B}-\mathrm{C} 8 \mathrm{~B}-\mathrm{C} 9 \mathrm{~B}-\mathrm{N} 1 \mathrm{~B}$ & $57.2(6)$ & $\mathrm{N} 4 \mathrm{C}-\mathrm{C} 16 \mathrm{C}-\mathrm{C} 17 \mathrm{C}-\mathrm{O} 8 \mathrm{C}$ & $-168.7(4)$ \\
\hline $\mathrm{C} 10 \mathrm{~B}-\mathrm{N} 1 \mathrm{~B}-\mathrm{C} 9 \mathrm{~B}-\mathrm{C} 8 \mathrm{~B}$ & $-159.2(4)$ & N4C-C16C-C17C-O7C & $12.0(6)$ \\
\hline $\mathrm{C} 1 \mathrm{~B}-\mathrm{N} 1 \mathrm{~B}-\mathrm{C} 9 \mathrm{~B}-\mathrm{C} 8 \mathrm{~B}$ & $83.6(5)$ & & \\
\hline $\mathrm{Zr} 2-\mathrm{N} 1 \mathrm{~B}-\mathrm{C} 9 \mathrm{~B}-\mathrm{C} 8 \mathrm{~B}$ & $-49.3(5)$ & & \\
\hline
\end{tabular}


Table S5. Hydrogen-bond geometry ( $\AA$, ํ)

\begin{tabular}{|c|c|c|c|c|}
\hline$D-\mathrm{H} \cdots A$ & $D-\mathrm{H}$ & $\mathrm{H} \cdots A$ & $D \cdots A$ & $D-\mathrm{H} \cdots A$ \\
\hline $\mathrm{O} 1-\mathrm{H} 11 \cdots \mathrm{O} 8 A^{\mathrm{i}}$ & $0.82(1)$ & $1.95(2)$ & $2.755(6)$ & $170(5)$ \\
\hline $\mathrm{O} 1-\mathrm{H} 12 \cdots \mathrm{O} 4 B^{\mathrm{ii}}$ & $0.82(1)$ & $2.00(3)$ & $2.782(6)$ & $159(7)$ \\
\hline $\mathrm{O} 2-\mathrm{H} 21 \cdots \mathrm{O} 1$ & $0.83(2)$ & $2.06(2)$ & $2.881(7)$ & $171(7)$ \\
\hline $\mathrm{O} 2-\mathrm{H} 22 \cdots \mathrm{O} 2 B^{\mathrm{ii}}$ & $0.82(2)$ & $2.05(2)$ & $2.865(6)$ & $171(7)$ \\
\hline $\mathrm{O} 3-\mathrm{H} 31 \cdots \mathrm{O} 4$ & $0.81(2)$ & $1.88(2)$ & $2.685(6)$ & $170(8)$ \\
\hline 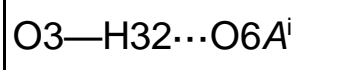 & $0.81(1)$ & $1.93(2)$ & $2.736(5)$ & $174(5)$ \\
\hline $\mathrm{O} 4-\mathrm{H} 41 \cdots \mathrm{O} 5^{\mathrm{iii}}$ & $0.82(2)$ & $1.94(3)$ & $2.733(6)$ & $160(9)$ \\
\hline $\mathrm{O} 4-\mathrm{H} 42 \cdots \mathrm{O} 4 A^{\mathrm{iv}}$ & $0.82(2)$ & $1.96(2)$ & $2.760(5)$ & $166(6)$ \\
\hline $\mathrm{O} 5-\mathrm{H} 51 \cdots \mathrm{O} 3$ & $0.81(1)$ & $1.97(2)$ & $2.772(6)$ & $170(4)$ \\
\hline $\mathrm{O} 5-\mathrm{H} 52 \cdots \mathrm{O}^{2} 0^{v}$ & $0.82(1)$ & $1.95(2)$ & $2.769(6)$ & $177(6)$ \\
\hline $\mathrm{O} 6-\mathrm{H} 61 \cdots \mathrm{O} 8 \mathrm{C}$ & $0.82(2)$ & $1.93(2)$ & $2.740(5)$ & $173(7)$ \\
\hline $\mathrm{O} 6-\mathrm{H} 62 \cdots \mathrm{O} 8 B^{\mathrm{iii}}$ & $0.81(1)$ & $2.02(2)$ & $2.824(6)$ & $169(7)$ \\
\hline $\mathrm{O} 7-\mathrm{H} 71 \cdots \mathrm{O} 6^{\mathrm{i}}$ & $0.83(1)$ & $2.08(3)$ & $2.881(7)$ & $163(8)$ \\
\hline $\mathrm{O} 7-\mathrm{H} 72 \cdots \mathrm{O} 6 \mathrm{~B}$ & $0.82(2)$ & $2.06(2)$ & $2.886(6)$ & $179(7)$ \\
\hline $\mathrm{O} 8-\mathrm{H} 81 \cdots \mathrm{O} 10$ & $0.81(2)$ & $1.98(2)$ & $2.775(6)$ & $165(6)$ \\
\hline $\mathrm{O} 8-\mathrm{H} 82 \cdots \mathrm{O}^{\mathrm{vi}}$ & $0.82(1)$ & $1.97(2)$ & $2.764(6)$ & $166(7)$ \\
\hline 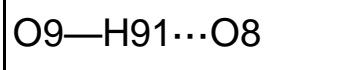 & $0.83(1)$ & $1.90(2)$ & $2.715(6)$ & $170(5)$ \\
\hline $\mathrm{O} 9-\mathrm{H} 92 \cdots \mathrm{O} 4 \mathrm{C}^{\mathrm{vii}}$ & $0.82(1)$ & $1.93(2)$ & $2.754(6)$ & $175(6)$ \\
\hline O10-H101‥O6C & $0.81(1)$ & $1.94(2)$ & $2.745(5)$ & $170(5)$ \\
\hline $\mathrm{O} 10-\mathrm{H} 102 \cdots \mathrm{O} 9^{\mathrm{iii}}$ & $0.82(2)$ & $1.91(3)$ & 2.699 (6) & $163(8)$ \\
\hline
\end{tabular}

Symmetry codes: (i) $x, y-1, z$; (ii) $x-1 / 2, y+1 / 2, z$; (iii) $x, y+1, z$; (iv) $x-1 / 2, y-1 / 2, z$; (v) $x,-y+2, z+1 / 2$; (vi) $x,-$ $y+2, z-1 / 2 ;$ (vii) $x+1 / 2, y-1 / 2, z$. 
Least-squares planes ( $x, y, z$ in crystal coordinates) and deviations from them (* indicates atom used to define plane)

$0.9419(0.0224) x+6.7776(0.0020) y-12.1401(0.0530) z=0.9781(0.0496)$

* $0.1240(0.0015) \mathrm{O} 1 \mathrm{~A}$

* $-0.1211(0.0015)$ O3A

* $0.1163(0.0014) \mathrm{O}$ A

* $\quad-0.1192(0.0015)$ O7A

$-1.0259(0.0015)$ Zr1

$-2.2041(0.0046) \mathrm{N} 1 \mathrm{~A}$

$-2.3020(0.0044) \mathrm{N} 2 \mathrm{~A}$

$-2.1551(0.0045) \mathrm{N} 3 \mathrm{~A}$

$-2.5963(0.0042) \mathrm{N} 4 \mathrm{~A}$

Rms deviation of fitted atoms $=0.1202$

$-1.1167(0.0222) x-6.6504(0.0027) y+15.3003(0.0578) z=4.2197(0.0581)$

Angle to previous plane (with approximate esd) $=3.785(0.087)$

* $-0.1315(0.0016) \mathrm{N} 1 \mathrm{~A}$

* $\quad 0.1273(0.0015) \mathrm{N} 2 \mathrm{~A}$

* $\quad-0.1540(0.0018) \mathrm{N} 3 \mathrm{~A}$

* $\quad 0.1582(0.0019) \mathrm{N} 4 \mathrm{~A}$

$-1.2851(0.0017) \mathrm{Zr} 1$

$-2.5070(0.0039) \mathrm{O} 1 \mathrm{~A}$

$-2.0899(0.0043)$ O3A

$-2.3459(0.0039) \mathrm{O} A$

$-2.2898(0.0040) \mathrm{O} A \mathrm{~A}$

Rms deviation of fitted atoms $=0.1434$ 
$-0.7072(0.0240) x+6.9685(0.0009) y-2.9665(0.0656) z=0.5485(0.0507)$

Angle to previous plane (with approximate esd) $=14.797(0.070)$

* $-0.1138(0.0014) \mathrm{O} 1 \mathrm{~B}$

* $\quad 0.1126(0.0014)$ O3B

* $-0.1103(0.0014)$ O5B

* $\quad 0.1116(0.0014)$ O7B

$1.0360(0.0014)$ Zr2

$2.2000(0.0051)$ N1B

$2.2925(0.0049)$ N2B

$2.1717(0.0049)$ N3B

$2.5748(0.0048) \mathrm{N} 4 \mathrm{~B}$

Rms deviation of fitted atoms $=0.1121$

$0.4842(0.0279) x+6.9620(0.0010) y-4.3223(0.0623) z=3.0083(0.0498)$

Angle to previous plane (with approximate esd) $=3.676(0.030)$

* $-0.1207(0.0018)$ N1B

* $\quad 0.1176(0.0018)$ N2B

* $-0.1407(0.0021)$ N3B

* $0.1438(0.0022) \mathrm{N} 4 \mathrm{~B}$

$-1.2693(0.0019) \mathrm{Zr} 2$

$-2.4853(0.0042) \mathrm{O} 1 \mathrm{~B}$

-2.0945 (0.0046) O3B

$-2.3433(0.0041)$ O5B

$-2.2898(0.0043)$ O7B

Rms deviation of fitted atoms $=0.1312$ 
$-0.7622(0.0214) x+6.7766(0.0019) y+12.1758(0.0519) z=14.1519(0.0288)$

Angle to previous plane (with approximate esd) $=19.143(0.049)$

* $0.1226(0.0015) \mathrm{O} \mathrm{C}$

* $-0.1195(0.0015) \mathrm{O3C}$

* $0.1149(0.0014) \mathrm{O} 5 \mathrm{C}$

* $-0.1180(0.0015) \mathrm{O} 7 \mathrm{C}$

$-1.0270(0.0015)$ Zr3

$-2.2074(0.0044) \mathrm{N} 1 \mathrm{C}$

$-2.3131(0.0044) \mathrm{N} 2 \mathrm{C}$

$-2.1569(0.0045)$ N3C

$-2.5896(0.0041) \mathrm{N} 4 \mathrm{C}$

Rms deviation of fitted atoms $=0.1188$

$-0.9408(0.0219) x+6.6587(0.0026) y+15.1199(0.0565) z=13.3718(0.0336)$

Angle to previous plane (with approximate esd) $=3.524(0.085)$

* $0.1312(0.0016) \mathrm{N} 1 \mathrm{C}$

* $-0.1265(0.0015) \mathrm{N} 2 \mathrm{C}$

* $0.1530(0.0018) \mathrm{N} 3 \mathrm{C}$

* $-0.1577(0.0019) \mathrm{N} 4 \mathrm{C}$

$1.2864(0.0017) \mathrm{Zr3}$

$2.5042(0.0037) \mathrm{O} 1 \mathrm{C}$

2.1027 (0.0042) O3C

$2.3521(0.0040)$ O5C

2.2854 (0.0039) O7C

Rms deviation of fitted atoms $=0.1428$ 


\section{Crystal Structure Analysis of $\mathrm{Zr}-\mathrm{PCTA},\left[\mathrm{Zr}\left(\mathrm{C}_{17} \mathrm{H}_{21} \mathrm{~N}_{4} \mathrm{O}_{6}\right)\right]_{2} \mathrm{O}-8 \mathrm{H}_{2} \mathrm{O}$}

\section{Crystallographic Experimental Details:}

Data Collection and Structure Solution. A clear colourless plate-like specimen of $\left[\mathrm{Zr}\left(\mathrm{C}_{17} \mathrm{H}_{21} \mathrm{~N}_{4} \mathrm{O}_{6}\right)\right]_{2} \mathrm{O}-8 \mathrm{H}_{2} \mathrm{O}$, approximate dimensions $0.030 \mathrm{~mm} \times 0.098 \mathrm{~mm} \times 0.460 \mathrm{~mm}$, was used for the X-ray crystallographic analysis. The X-ray intensity data were measured on a Bruker APEX CCD system equipped with a graphite monochromator and a Mo Ka sealed $x$-ray tube $(\lambda=0.71073 \AA)$. X-rays were provided by a fine-focus sealed $x$ ray tube operated at $50 \mathrm{kV}$ and $30 \mathrm{~mA}$.

The total exposure time was 18.65 hours. The frames were integrated with the Bruker SAINT Software ${ }^{1}$ package using a narrow-frame algorithm. The integration of the data using a monoclinic unit cell yielded a total of 39,418 reflections to a maximum $\theta$ angle of $30.08^{\circ}(0.71 \AA$ resolution), of which 6124 were independent (average redundancy 6.437 , completeness $=99.2 \%, \mathrm{R}_{\text {int }}=4.67 \%, \mathrm{R}_{\mathrm{sig}}=2.93 \%$ ) and $5433(88.72 \%)$ were greater than $2 \sigma\left(F^{2}\right)$. The final cell constants of $a=26.632(4) \AA, b=10.6634(17) \AA, c=15.884(3) \AA, \beta=111.098(2)^{\circ}$, volume $=4208.5(12) \AA^{3}$, are based upon the refinement of the XYZ-centroids of 9951 reflections above $20 \sigma(\mathrm{I})$ with $6.877^{\circ}<2 \theta<60.06^{\circ}$. Data were corrected for absorption effects using the multi-scan method (SADABS) ${ }^{2}$. The ratio of minimum to maximum apparent transmission was 0.895 . The calculated minimum and maximum transmission coefficients (based on crystal size) are 0.7730 and 0.9830 .

The structure was solved and refined using the Bruker SHELXTL Software Package ${ }^{3}$, using the space group $\mathrm{C} 2 / \mathrm{c}$, with $\mathrm{Z}=4$ for the formula unit, $\mathrm{C}_{34} \mathrm{H}_{58} \mathrm{~N}_{8} \mathrm{O}_{21} \mathrm{Zr}_{2}$. The final model includes 4 "full" occupancy water molecules per asymmetric unit. The final structural model incorporated isotropic thermal parameters for all included hydrogen atoms. The hydrogen atoms of the PCTA ligand were included in the structural model as fixed atoms (using idealized $\mathrm{sp}^{2}$ - or $\mathrm{sp}^{3}$-hybridized geometry and $\mathrm{C}-\mathrm{H}$ bond lengths of 0.95 and $0.99 \AA$, respectively) "riding" on their respective carbon atoms. The isotropic thermal parameters for all PCTA hydrogen atoms were fixed at values 1.2 times the equivalent isotropic thermal parameter of the carbon atom to which they are covalently bonded. The isotropic thermal parameters of the water hydrogen atoms were allowed to refine independently. The final anisotropic full-matrix least-squares refinement on $\mathrm{F}^{2}$ with 326 variables converged at $\mathrm{R}_{1}=2.33 \%$, for the observed data and $w R_{2}=6.33 \%$ for all data. The goodness-of-fit was 1.057 . The largest peak in the final difference electron density synthesis was $0.529 \mathrm{e}^{-} / \AA^{3}$ and the largest hole was $-0.309 \mathrm{e}^{-} / \AA^{3}$ with an RMS deviation 
of $0.074 \mathrm{e}^{-} / \AA^{3}$. On the basis of the final model, the calculated density was $1.732 \mathrm{~g} / \mathrm{cm}^{3}$ and $F(000), 2264 \mathrm{e}$.

Refinement Details. Crystal data, data collection and structure refinement details are summarized in tables below.

Computing details. Data collection: Bruker APEX3 v2016.1-0; cell refinement: Bruker APEX3 v2016.1-0; data reduction: Bruker APEX2 v2014.11-0; program(s) used to solve structure: SHELXS97 (Sheldrick 2008); program(s) used to refine structure: SHELXL2014 (Sheldrick, 2014); molecular graphics: Bruker APEX2 v2014.11-0; software used to prepare material for publication: Bruker APEX2 v2014.11-01-6. 


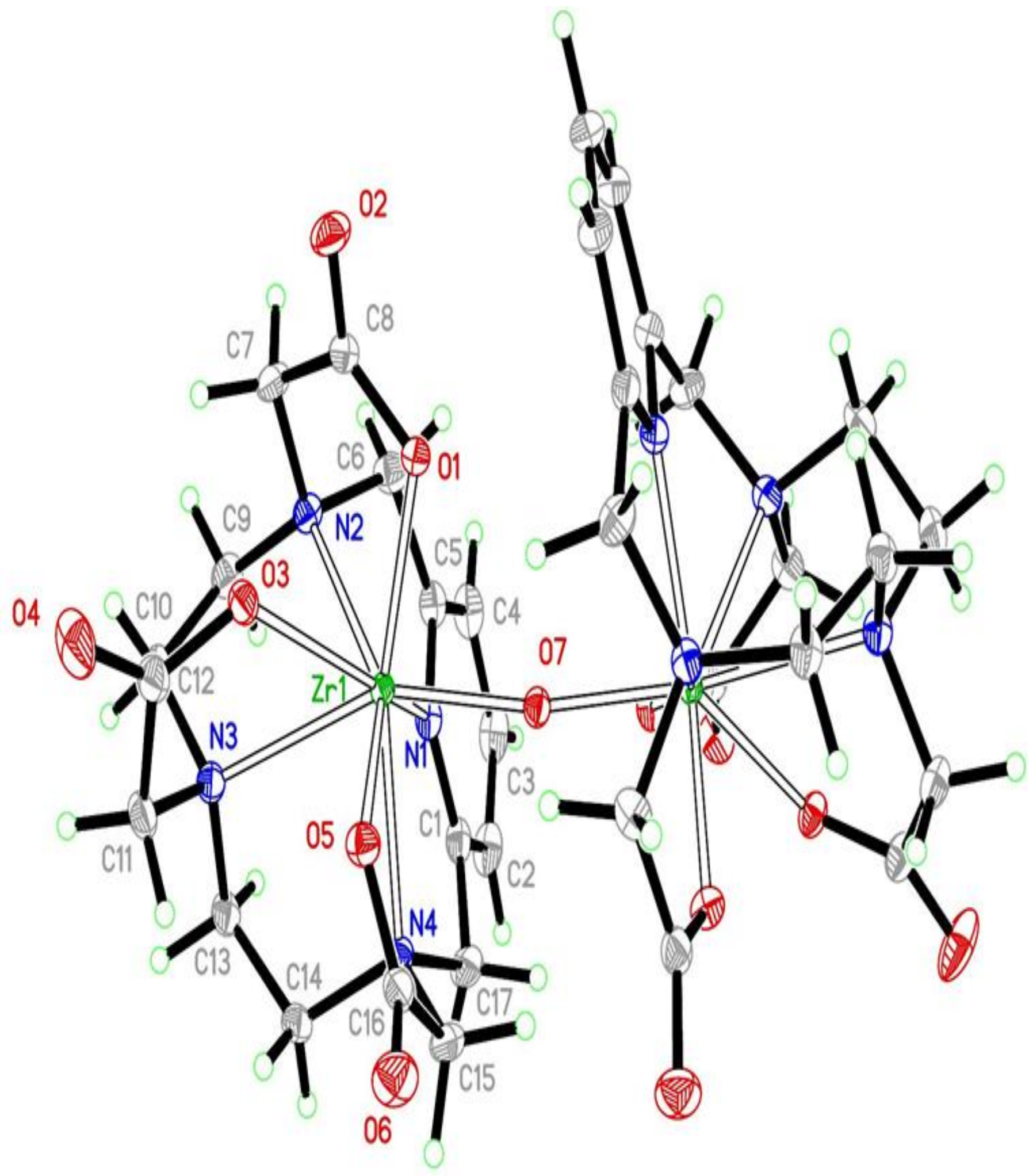

Figure $\mathrm{S} 31.50 \%$ probability plot for the dimeric unit of $\left[\mathrm{Zr}\left(\mathrm{C}_{17} \mathrm{H}_{21} \mathrm{~N}_{4} \mathrm{O}_{6}\right)\right]_{2} \mathrm{O}-\mathbf{8} \mathrm{H}_{2} \mathrm{O}$ with the water molecules omitted for clarity. The bridging oxygen atom, $\mathrm{O}_{7}$, lies on the crystallographic 2 -fold axis at $1 / 2, y, 1 / 4$ in the unit cell and only the non-hydrogen atoms of the asymmetric unit are labeled. 


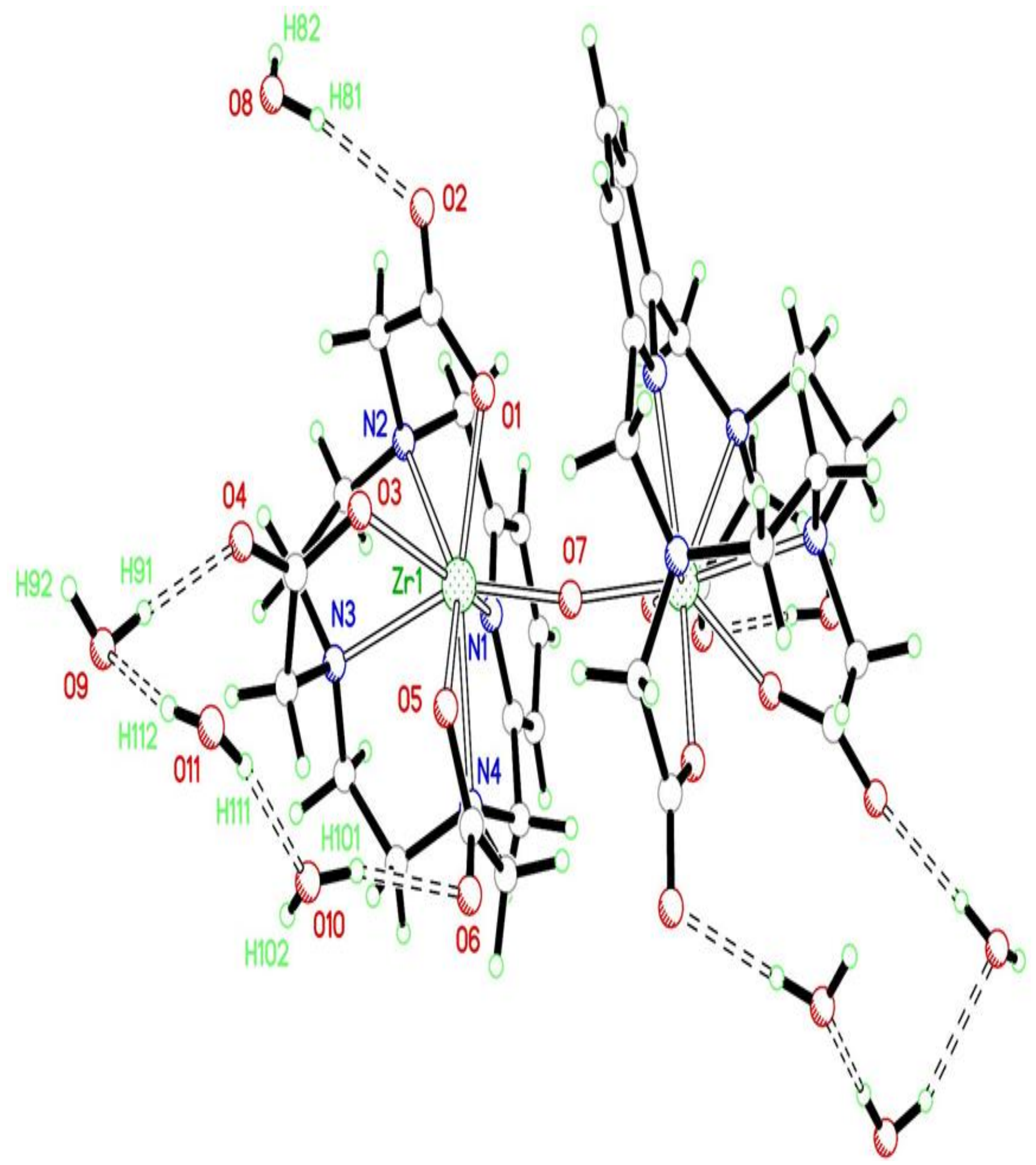

Figure S32. Plot of $\left[\mathrm{Zr}\left(\mathrm{C}_{17} \mathrm{H}_{21} \mathrm{~N}_{4} \mathrm{O}_{6}\right)\right]_{2} \mathrm{O}-8 \mathrm{H}_{2} \mathrm{O}$ with all atoms represented by dummy-sized spheres and hydrogen-bonding interactions represented by dashed-open bonds. 


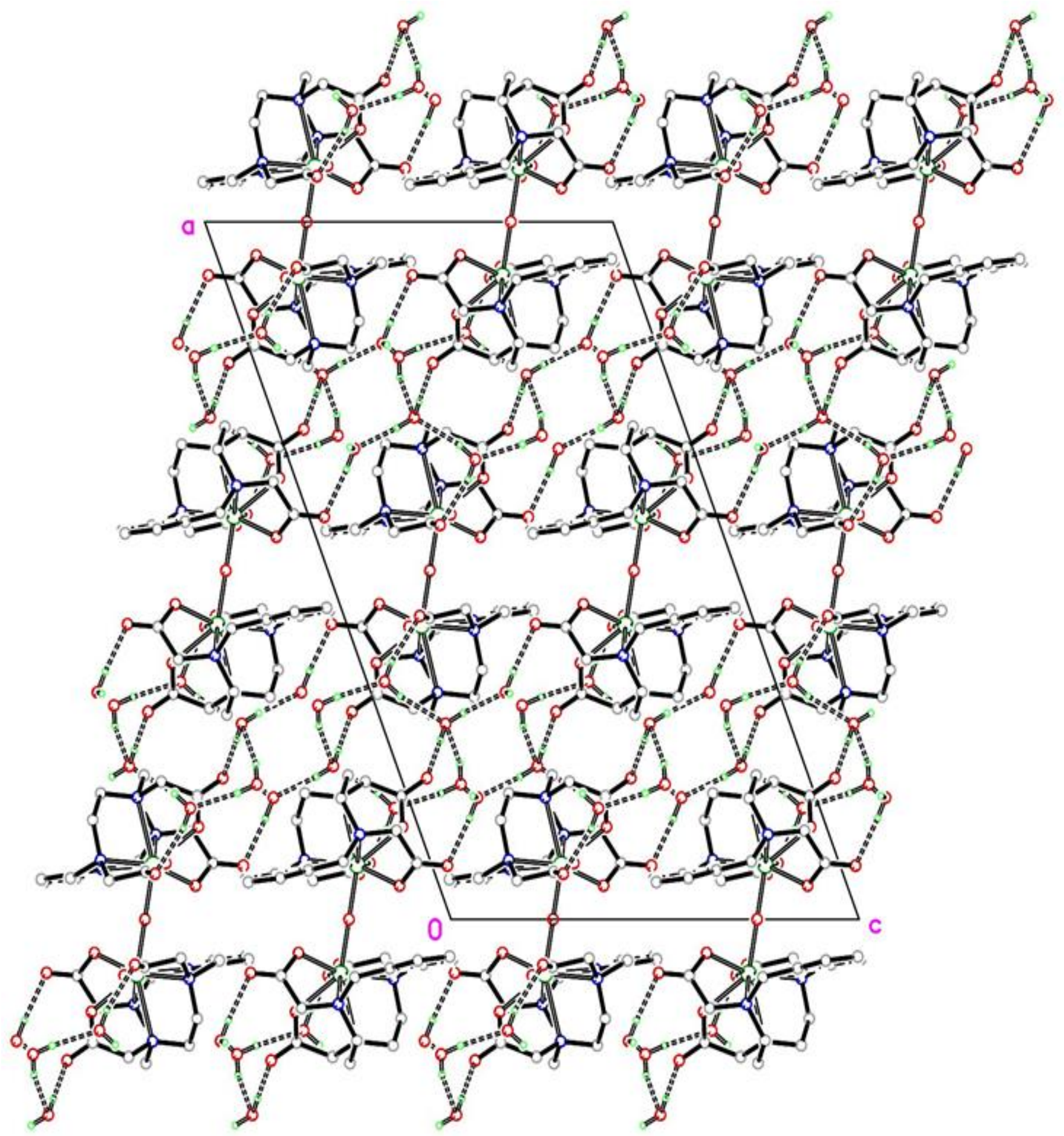

Figure S33. Packing plot of the solid-state structure of $\left[\mathrm{Zr}\left(\mathrm{C}_{17} \mathrm{H}_{21} \mathrm{~N}_{4} \mathrm{O}_{6}\right)\right]_{2} \mathrm{O}-8 \mathrm{H}_{2} \mathrm{O}$ viewed in projection down the $\mathbf{b}$ axis of the unit cell. All atoms are represented by dummy-sized spheres and non-water hydrogen atoms have been omitted for clarity. Hydrogen-bonding interactions represented by dashed-solid bonds. 


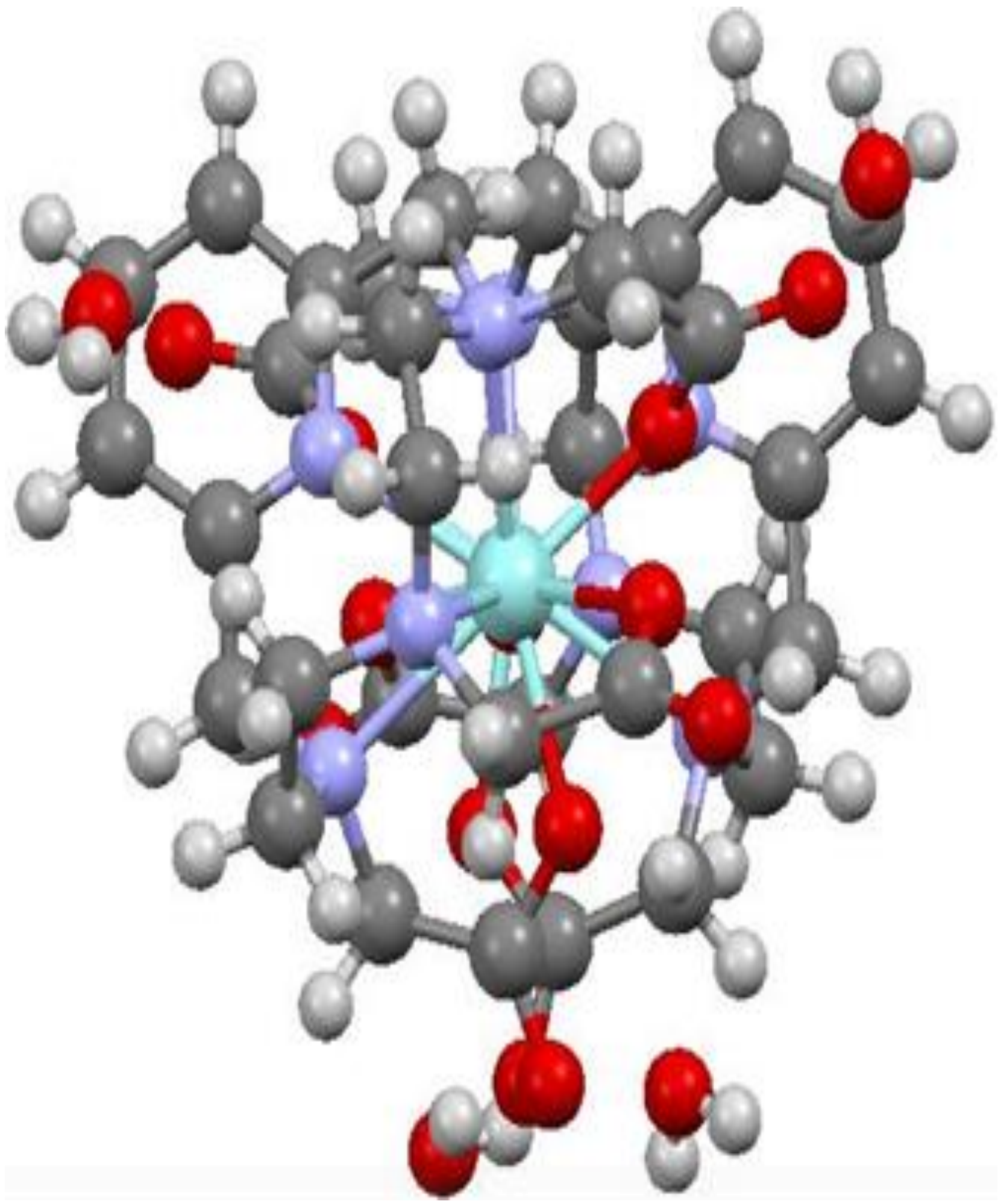

Figure S34. Plot of $\left[\mathrm{Zr}\left(\mathrm{C}_{17} \mathrm{H}_{21} \mathrm{~N}_{4} \mathrm{O}_{6}\right)\right]_{2} \mathrm{O}-8 \mathrm{H}_{2} \mathrm{O}$, viewed down the $\mathrm{Zr}$--- $\mathrm{Zr}$ vector with all atoms represented by dummy-sized spheres. 


\section{Crystal data}

\begin{tabular}{|l|l|}
\hline $\mathrm{C}_{34} \mathrm{H}_{58} \mathrm{~N}_{8} \mathrm{O}_{21} \mathrm{Zr}_{2}$ & $F(000)=2264$ \\
\hline$M_{r}=1097.32$ & $D_{x}=1.732 \mathrm{Mg} \mathrm{m}^{-3}$ \\
\hline Monoclinic, C2/c & Mo Ko radiation, $\lambda=0.71073 \AA$ \\
\hline$a=26.632(4) \AA$ & Cell parameters from 9951 reflections \\
\hline$b=10.6634(17) \AA$ & $\theta=3.4-30.0^{\circ}$ \\
\hline$C=15.884(3) \AA$ & $\mu=0.59 \mathrm{~mm}^{-1}$ \\
\hline$\beta=111.098(2)^{\circ}$ & $T=193 \mathrm{~K}$ \\
\hline$V=4208.6(12) \AA^{3}$ & $P l a t e$, colourless \\
\hline$Z=4$ & $0.46 \times 0.10 \times 0.03 \mathrm{~mm}$ \\
\hline
\end{tabular}

\section{Data collection}

\begin{tabular}{|l|l|}
\hline Bruker APEX CCD diffractometer & 6124 independent reflections \\
\hline Radiation source: sealed tube & 5433 reflections with $I>2 \sigma(I)$ \\
\hline Graphite monochromator & $R_{\text {int }}=0.047$ \\
\hline$\phi$ and $\omega$ scans & $\theta_{\max }=30.1^{\circ}, \theta_{\min }=3.4^{\circ}$ \\
\hline $\begin{array}{l}\text { Absorption correction: multi-scan Data were } \\
\text { corrected for scaling and absorption effects using } \\
\text { the multi-scan technique (SADABS). The ratio of } \\
\text { minimum to maximum apparent transmission was } \\
0.895 . \text { The calculated minimum and maximum } \\
\text { transmission coefficients (based on crystal size) are } \\
0.773 \text { and } 0.983 .\end{array}$ & $h=-37 \rightarrow 37$ \\
\hline$T_{\text {min }}=0.667, T_{\max }=0.746$ & $k=-14 \rightarrow 14$ \\
\hline 39418 measured reflections & $I=-22 \rightarrow 22$ \\
\hline
\end{tabular}




\section{Refinement}

\begin{tabular}{|l|l|}
\hline Refinement on $F^{2}$ & 12 restraints \\
\hline Least-squares matrix: full & Hydrogen site location: mixed \\
\hline$R\left[F^{2}>2 \sigma\left(F^{2}\right)\right]=0.023$ & $\begin{array}{l}\text { H atoms treated by a mixture of independent and } \\
\text { constrained refinement }\end{array}$ \\
\hline$w R\left(F^{2}\right)=0.063$ & $\begin{array}{l}W=1 /\left[\sigma^{2}\left(F_{0}^{2}\right)+(0.0312 P)^{2}+2.5169 P\right] \\
\text { where } P=\left(F_{0}^{2}+2 F_{\mathrm{c}}^{2}\right) / 3\end{array}$ \\
\hline$S=1.06$ & $(\Delta / \sigma)_{\max }=0.002$ \\
\hline 6124 reflections & $\Delta\rangle_{\max }=0.53$ e $\AA^{-3}$ \\
\hline 326 parameters & $\Delta\rangle_{\min }=-0.31$ e $\AA^{-3}$ \\
\hline
\end{tabular}

\section{Special details}

Geometry. All esds (except the esd in the dihedral angle between two I.s. planes) are estimated using the full covariance matrix. The cell esds are taken into account individually in the estimation of esds in distances, angles and torsion angles; correlations between esds in cell parameters are only used when they are defined by crystal symmetry. An approximate (isotropic) treatment of cell esds is used for estimating esds involving I.s. planes. 
Table S7. Fractional atomic coordinates and isotropic or equivalent isotropic displacement parameters $\left(\AA^{2}\right)$

\begin{tabular}{|c|c|c|c|c|}
\hline & $x$ & $y$ & $z$ & $U_{\text {iso }}{ }^{*} / U_{\text {eq }}$ \\
\hline Zr1 & $0.42185(2)$ & $0.39753(2)$ & 0.18239 (2) & $0.00952(4)$ \\
\hline 01 & 0.45174 (4) & $0.28434(8)$ & $0.09702(6)$ & $0.01517(17)$ \\
\hline $\mathrm{O} 2$ & 0.42315 (4) & $0.18849(9)$ & $-0.03754(7)$ & $0.0218(2)$ \\
\hline O3 & 0.36737 (4) & $0.42299(8)$ & $0.03892(6)$ & $0.01564(18)$ \\
\hline $\mathrm{O} 4$ & $0.29752(5)$ & $0.51715(12)$ & $-0.06308(7)$ & $0.0342(3)$ \\
\hline O5 & 0.41950 (4) & $0.59413(8)$ & $0.15041(7)$ & $0.01590(18)$ \\
\hline 06 & $0.43478(5)$ & $0.79600(9)$ & $0.18580(7)$ & $0.0258(2)$ \\
\hline $\mathrm{O} 7$ & 0.5000 & $0.41805(11)$ & 0.2500 & $0.0129(2)$ \\
\hline N1 & $0.42802(4)$ & $0.27938(10)$ & $0.31193(7)$ & $0.0141(2)$ \\
\hline $\mathrm{N} 2$ & 0.37848 (4) & $0.19029(10)$ & $0.15047(7)$ & $0.0146(2)$ \\
\hline N3 & $0.32502(4)$ & 0.41969 (10) & $0.16303(7)$ & $0.0147(2)$ \\
\hline N4 & $0.41500(4)$ & $0.53215(10)$ & $0.30742(7)$ & $0.0138(2)$ \\
\hline C1 & $0.43550(5)$ & $0.33262(12)$ & 0.39228 (9) & $0.0162(2)$ \\
\hline C2 & $0.44272(6)$ & $0.26188(14)$ & $0.46930(9)$ & $0.0215(3)$ \\
\hline $\mathrm{H} 2$ & 0.4449 & 0.3014 & 0.5241 & $0.026^{*}$ \\
\hline C3 & $0.44668(6)$ & $0.13323(15)$ & $0.46466(10)$ & 0.0241 (3) \\
\hline H3 & 0.4533 & 0.0833 & 0.5171 & $0.029^{*}$ \\
\hline C4 & $0.44087(6)$ & $0.07749(13)$ & $0.38275(10)$ & $0.0221(3)$ \\
\hline $\mathrm{H} 4$ & 0.4444 & -0.0107 & 0.3785 & $0.027^{\star}$ \\
\hline C5 & $0.42986(5)$ & $0.15307(12)$ & $0.30748(9)$ & $0.0166(2)$ \\
\hline C6 & $0.41690(6)$ & $0.10220(11)$ & $0.21398(10)$ & 0.0187 (3) \\
\hline $\mathrm{H} 6 \mathrm{~A}$ & 0.4501 & 0.0954 & 0.1998 & $0.022^{*}$ \\
\hline H6B & 0.4006 & 0.0178 & 0.2090 & $0.022^{*}$ \\
\hline C7 & $0.37287(6)$ & $0.15697(12)$ & $0.05720(9)$ & 0.0176 (2) \\
\hline H7A & 0.3383 & 0.1894 & 0.0144 & $0.021^{*}$ \\
\hline H7B & 0.3729 & 0.0646 & 0.0509 & $0.021^{*}$ \\
\hline C8 & 0.41929 (5) & $0.21323(11)$ & $0.03532(9)$ & $0.0156(2)$ \\
\hline
\end{tabular}




\begin{tabular}{|c|c|c|c|c|}
\hline C9 & $0.32621(5)$ & 0.18499 (12) & $0.16450(9)$ & $0.0179(2)$ \\
\hline H9A & 0.3329 & 0.1771 & 0.2297 & $0.021^{*}$ \\
\hline H9B & 0.3057 & 0.1104 & 0.1335 & $0.021^{*}$ \\
\hline C10 & $0.29368(5)$ & 0.30206 (12) & $0.12784(9)$ & $0.0182(2)$ \\
\hline $\mathrm{H} 10 \mathrm{~A}$ & 0.2816 & 0.3015 & 0.0612 & $0.022^{*}$ \\
\hline $\mathrm{H} 10 \mathrm{~B}$ & 0.2612 & 0.3016 & 0.1444 & $0.022^{*}$ \\
\hline C11 & $0.30270(5)$ & 0.51515 (12) & $0.09072(9)$ & $0.0182(2)$ \\
\hline $\mathrm{H} 11 \mathrm{~A}$ & 0.3145 & 0.6001 & 0.1149 & $0.022^{*}$ \\
\hline H11B & 0.2629 & 0.5126 & 0.0676 & $0.022^{*}$ \\
\hline C12 & $0.32331(6)$ & $0.48514(12)$ & $0.01498(9)$ & $0.0182(2)$ \\
\hline C13 & $0.31933(5)$ & $0.45841(12)$ & $0.24909(9)$ & $0.0169(2)$ \\
\hline $\mathrm{H} 13 \mathrm{~A}$ & 0.3272 & 0.3862 & 0.2910 & $0.020^{*}$ \\
\hline H13B & 0.2819 & 0.4856 & 0.2372 & $0.020^{*}$ \\
\hline C14 & $0.35762(5)$ & 0.56506 (12) & $0.29194(9)$ & $0.0169(2)$ \\
\hline $\mathrm{H} 14 \mathrm{~A}$ & 0.3472 & 0.6396 & 0.2523 & $0.020^{*}$ \\
\hline H14B & 0.3544 & 0.5871 & 0.3504 & $0.020^{*}$ \\
\hline C15 & $0.44408(6)$ & $0.64965(12)$ & $0.30442(9)$ & $0.0177(2)$ \\
\hline $\mathrm{H} 15 \mathrm{~A}$ & 0.4833 & 0.6369 & 0.3350 & $0.021^{*}$ \\
\hline $\mathrm{H} 15 \mathrm{~B}$ & 0.4327 & 0.7173 & 0.3363 & $0.021^{*}$ \\
\hline C16 & $0.43185(5)$ & $0.68670(11)$ & $0.20747(9)$ & $0.0163(2)$ \\
\hline C17 & $0.44095(6)$ & 0.47195 (12) & $0.39679(8)$ & 0.0184 (3) \\
\hline $\mathrm{H} 17 \mathrm{~A}$ & 0.4244 & 0.5050 & 0.4390 & $0.022^{*}$ \\
\hline H17B & 0.4796 & 0.4943 & 0.4209 & $0.022^{*}$ \\
\hline O8 & $0.32558(5)$ & $0.09800(12)$ & $-0.17010(9)$ & $0.0361(3)$ \\
\hline H81 & $0.3553(5)$ & $0.1162(18)$ & $-0.1327(12)$ & $0.043(6)^{*}$ \\
\hline H82 & $0.3235(10)$ & $0.0224(10)$ & $-0.1609(17)$ & $0.064(8)^{*}$ \\
\hline O9 & $0.21960(5)$ & $0.68121(11)$ & $-0.15600(8)$ & $0.0306(2)$ \\
\hline H91 & $0.2423(7)$ & 0.6294 (17) & $-0.1299(12)$ & $0.045(6)^{*}$ \\
\hline H92 & $0.2044(7)$ & 0.6564 (18) & $-0.2071(7)$ & $0.034(5)^{*}$ \\
\hline
\end{tabular}




\begin{tabular}{|l|l|l|l|l|}
\hline $\mathrm{O} 10$ & $0.34067(6)$ & $0.84749(13)$ & $0.04255(9)$ & $0.0370(3)$ \\
\hline $\mathrm{H} 101$ & $0.3696(5)$ & $0.824(2)$ & $0.0776(13)$ & $0.054(7)^{\star}$ \\
\hline $\mathrm{H} 102$ & $0.3198(7)$ & $0.836(2)$ & $0.0690(15)$ & $0.057(7)^{\star}$ \\
\hline $\mathrm{O} 11$ & $0.30641(5)$ & $0.84782(12)$ & $-0.14118(9)$ & $0.0330(3)$ \\
\hline $\mathrm{H} 111$ & $0.3190(8)$ & $0.840(2)$ & $-0.0863(6)$ & $0.043(6)^{\star}$ \\
\hline $\mathrm{H} 112$ & $0.2771(5)$ & $0.814(2)$ & $-0.1565(14)$ & $0.049(7)^{\star}$ \\
\hline
\end{tabular}

Table S8. Atomic displacement parameters $\left(\AA^{2}\right)$

\begin{tabular}{|l|l|l|l|l|l|l|}
\hline & $U^{11}$ & $U^{22}$ & $U^{\beta 3}$ & $U^{12}$ & $U^{13}$ & $U^{23}$ \\
\hline Zr1 & $0.00958(6)$ & $0.01080(6)$ & $0.00801(6)$ & $0.00027(4)$ & $0.00296(4)$ & $-0.00017(3)$ \\
\hline O1 & $0.0130(4)$ & $0.0175(4)$ & $0.0151(4)$ & $0.0003(3)$ & $0.0051(3)$ & $-0.0040(3)$ \\
\hline O2 & $0.0247(5)$ & $0.0266(5)$ & $0.0160(5)$ & $-0.0040(4)$ & $0.0096(4)$ & $-0.0071(4)$ \\
\hline O3 & $0.0152(5)$ & $0.0196(4)$ & $0.0118(4)$ & $0.0037(3)$ & $0.0044(4)$ & $0.0007(3)$ \\
\hline O4 & $0.0333(6)$ & $0.0523(7)$ & $0.0144(5)$ & $0.0219(5)$ & $0.0053(5)$ & $0.0083(5)$ \\
\hline O5 & $0.0187(5)$ & $0.0141(4)$ & $0.0147(5)$ & $0.0014(3)$ & $0.0057(4)$ & $0.0009(3)$ \\
\hline O6 & $0.0315(6)$ & $0.0151(4)$ & $0.0296(6)$ & $-0.0015(4)$ & $0.0093(5)$ & $0.0012(4)$ \\
\hline O7 & $0.0120(6)$ & $0.0141(5)$ & $0.0120(6)$ & 0.000 & $0.0037(5)$ & 0.000 \\
\hline N1 & $0.0128(5)$ & $0.0171(5)$ & $0.0127(5)$ & $-0.0004(4)$ & $0.0049(4)$ & $0.0021(4)$ \\
\hline N2 & $0.0154(5)$ & $0.0155(5)$ & $0.0133(5)$ & $-0.0019(4)$ & $0.0055(4)$ & $-0.0013(4)$ \\
\hline N3 & $0.0134(5)$ & $0.0184(5)$ & $0.0127(5)$ & $0.0006(4)$ & $0.0052(4)$ & $-0.0010(4)$ \\
\hline N4 & $0.0135(5)$ & $0.0162(5)$ & $0.0118(5)$ & $-0.0009(4)$ & $0.0047(4)$ & $-0.0015(4)$ \\
\hline C1 & $0.0124(6)$ & $0.0227(6)$ & $0.0130(6)$ & $0.0003(4)$ & $0.0041(5)$ & $0.0017(4)$ \\
\hline C2 & $0.0185(7)$ & $0.0334(7)$ & $0.0122(6)$ & $-0.0025(5)$ & $0.0050(5)$ & $0.0041(5)$ \\
\hline C3 & $0.0200(7)$ & $0.0324(7)$ & $0.0176(7)$ & $-0.0025(5)$ & $0.0038(5)$ & $0.0113(5)$ \\
\hline C4 & $0.0187(7)$ & $0.0220(6)$ & $0.0244(7)$ & $-0.0004(5)$ & $0.0064(6)$ & $0.0086(5)$ \\
\hline C5 & $0.0144(6)$ & $0.0176(6)$ & $0.0173(6)$ & $-0.0005(4)$ & $0.0052(5)$ & $0.0034(4)$ \\
\hline C6 & $0.0239(7)$ & $0.0129(5)$ & $0.0192(7)$ & $0.0020(4)$ & $0.0075(5)$ & $0.0014(4)$ \\
\hline C7 & $0.0197(6)$ & $0.0181(6)$ & $0.0152(6)$ & $-0.0038(5)$ & $0.0064(5)$ & $-0.0053(4)$ \\
\hline C8 & $0.0156(6)$ & $0.0156(5)$ & $0.0148(6)$ & $0.0016(4)$ & $0.0047(5)$ & $-0.0017(4)$ \\
\hline
\end{tabular}




\begin{tabular}{|l|l|l|l|l|l|l|}
\hline C9 & $0.0169(6)$ & $0.0200(6)$ & $0.0181(6)$ & $-0.0055(5)$ & $0.0082(5)$ & $-0.0018(5)$ \\
\hline C10 & $0.0124(6)$ & $0.0241(6)$ & $0.0174(6)$ & $-0.0033(5)$ & $0.0045(5)$ & $-0.0033(5)$ \\
\hline C11 & $0.0158(6)$ & $0.0229(6)$ & $0.0148(6)$ & $0.0065(5)$ & $0.0044(5)$ & $0.0015(5)$ \\
\hline C12 & $0.0179(6)$ & $0.0207(6)$ & $0.0146(6)$ & $0.0037(5)$ & $0.0044(5)$ & $0.0004(4)$ \\
\hline C13 & $0.0139(6)$ & $0.0239(6)$ & $0.0148(6)$ & $0.0008(4)$ & $0.0074(5)$ & $-0.0010(4)$ \\
\hline C14 & $0.0159(6)$ & $0.0205(6)$ & $0.0159(6)$ & $0.0025(5)$ & $0.0077(5)$ & $-0.0028(5)$ \\
\hline C15 & $0.0204(6)$ & $0.0158(5)$ & $0.0184(6)$ & $-0.0034(5)$ & $0.0088(5)$ & $-0.0054(4)$ \\
\hline C16 & $0.0139(6)$ & $0.0145(5)$ & $0.0206(6)$ & $0.0012(4)$ & $0.0064(5)$ & $-0.0003(4)$ \\
\hline C17 & $0.0217(7)$ & $0.0222(6)$ & $0.0095(6)$ & $0.0006(5)$ & $0.0035(5)$ & $-0.0016(4)$ \\
\hline O8 & $0.0294(7)$ & $0.0374(7)$ & $0.0299(7)$ & $-0.0040(5)$ & $-0.0034(5)$ & $0.0022(5)$ \\
\hline 09 & $0.0272(6)$ & $0.0316(6)$ & $0.0269(6)$ & $0.0093(5)$ & $0.0024(5)$ & $0.0008(5)$ \\
\hline O10 & $0.0359(7)$ & $0.0451(7)$ & $0.0289(7)$ & $0.0038(6)$ & $0.0103(6)$ & $0.0094(5)$ \\
\hline O11 & $0.0315(7)$ & $0.0420(7)$ & $0.0271(6)$ & $-0.0052(5)$ & $0.0125(5)$ & $0.0011(5)$ \\
\hline
\end{tabular}

Table S9. Geometric parameters ( $\left.\AA^{\circ}, \stackrel{\circ}{)}\right)$

\begin{tabular}{|l|l|l|l|}
\hline $\mathrm{Zr} 1-\mathrm{O} 7$ & $1.9809(3)$ & $\mathrm{C} 4-\mathrm{H} 4$ & 0.9500 \\
\hline $\mathrm{Zr} 1-\mathrm{O} 5$ & $2.1527(9)$ & $\mathrm{C} 5-\mathrm{C} 6$ & $1.5000(19)$ \\
\hline $\mathrm{Zr} 1-\mathrm{O} 1$ & $2.1695(9)$ & $\mathrm{C} 6-\mathrm{H} 6 \mathrm{~A}$ & 0.9900 \\
\hline $\mathrm{Zr} 1-\mathrm{O} 3$ & $2.2343(10)$ & $\mathrm{C} 6-\mathrm{H} 6 \mathrm{~B}$ & 0.9900 \\
\hline $\mathrm{Zr} 1-\mathrm{N} 1$ & $2.3674(11)$ & $\mathrm{C} 7-\mathrm{C} 8$ & $1.5228(18)$ \\
\hline $\mathrm{Zr} 1-\mathrm{N} 2$ & $2.4603(11)$ & $\mathrm{C} 7-\mathrm{H} 7 \mathrm{~A}$ & 0.9900 \\
\hline $\mathrm{Zr} 1-\mathrm{N} 3$ & $2.4959(12)$ & $\mathrm{C}$-H7B & 0.9900 \\
\hline $\mathrm{Zr} 1-\mathrm{N} 4$ & $2.5097(11)$ & $\mathrm{C}-\mathrm{C} 10$ & $1.5115(19)$ \\
\hline $\mathrm{O} 1-\mathrm{C} 8$ & $1.2932(15)$ & $\mathrm{C}-\mathrm{H} 9 \mathrm{~A}$ & 0.9900 \\
\hline $\mathrm{O} 2-\mathrm{C} 8$ & $1.2269(16)$ & $\mathrm{C} 9-\mathrm{H} 9 \mathrm{~B}$ & 0.9900 \\
\hline $\mathrm{O} 3-\mathrm{C} 12$ & $1.2805(16)$ & $\mathrm{C} 10-\mathrm{H} 10 \mathrm{~A}$ & 0.9900 \\
\hline $\mathrm{O} 4-\mathrm{C} 12$ & $1.2298(17)$ & $\mathrm{C} 10-\mathrm{H} 10 \mathrm{~B}$ & 0.9900 \\
\hline $\mathrm{O} 5-\mathrm{C} 16$ & $1.2998(15)$ & $\mathrm{C} 11-\mathrm{C} 12$ & $1.5253(18)$ \\
\hline
\end{tabular}




\begin{tabular}{|c|c|c|c|}
\hline O6-C16 & $1.2257(16)$ & $\mathrm{C} 11-\mathrm{H} 11 \mathrm{~A}$ & 0.9900 \\
\hline $\mathrm{O} 7-Z \mathrm{Zr} 1^{\mathrm{i}}$ & $1.9810(3)$ & $\mathrm{C} 11-\mathrm{H} 11 \mathrm{~B}$ & 0.9900 \\
\hline $\mathrm{N} 1-\mathrm{C} 1$ & $1.3445(17)$ & $\mathrm{C} 13-\mathrm{C} 14$ & $1.5154(19)$ \\
\hline $\mathrm{N} 1-\mathrm{C} 5$ & $1.3506(16)$ & $\mathrm{C} 13-\mathrm{H} 13 \mathrm{~A}$ & 0.9900 \\
\hline $\mathrm{N} 2-\mathrm{C} 7$ & $1.4779(16)$ & $\mathrm{C} 13-\mathrm{H} 13 \mathrm{~B}$ & 0.9900 \\
\hline N2-C6 & $1.4845(17)$ & $\mathrm{C} 14-\mathrm{H} 14 \mathrm{~A}$ & 0.9900 \\
\hline $\mathrm{N} 2-\mathrm{C} 9$ & $1.4879(16)$ & C14-H14B & 0.9900 \\
\hline N3-C13 & $1.4870(16)$ & C15-C16 & $1.5076(19)$ \\
\hline N3-C11 & $1.4890(17)$ & C15-H15A & 0.9900 \\
\hline N3-C10 & $1.4988(17)$ & C15-H15B & 0.9900 \\
\hline N4-C15 & $1.4827(16)$ & C17-H17A & 0.9900 \\
\hline N4-C17 & $1.4829(17)$ & C17-H17B & 0.9900 \\
\hline $\mathrm{N} 4-\mathrm{C} 14$ & 1.4995 (17) & $\mathrm{O} 8-\mathrm{H} 81$ & $0.823(9)$ \\
\hline $\mathrm{C} 1-\mathrm{C} 2$ & 1.3905 (18) & O8-H82 & $0.824(9)$ \\
\hline C1-C17 & $1.4920(19)$ & $\mathrm{O9}-\mathrm{H} 91$ & $0.813(9)$ \\
\hline C2-C3 & $1.380(2)$ & $\mathrm{O} 9-\mathrm{H} 92$ & $0.811(9)$ \\
\hline $\mathrm{C} 2-\mathrm{H} 2$ & 0.9500 & $\mathrm{O} 10-\mathrm{H} 101$ & $0.811(9)$ \\
\hline C3-C4 & $1.388(2)$ & $\mathrm{O} 10-\mathrm{H} 102$ & $0.816(9)$ \\
\hline C3-H3 & 0.9500 & $\mathrm{O} 11-\mathrm{H} 111$ & $0.818(9)$ \\
\hline C4-C5 & $1.3830(18)$ & $\mathrm{O} 11-\mathrm{H} 112$ & $0.813(9)$ \\
\hline O7-Zr1-O5 & $87.48(4)$ & N2-C6-C5 & $107.50(10)$ \\
\hline $\mathrm{O} 7-\mathrm{Zr} 1-\mathrm{O} 1$ & 81.37 (3) & $\mathrm{N} 2-\mathrm{C} 6-\mathrm{H} 6 \mathrm{~A}$ & 110.2 \\
\hline O5-Zr1-O1 & 112.35 (4) & $\mathrm{C} 5-\mathrm{C} 6-\mathrm{H} 6 \mathrm{~A}$ & 110.2 \\
\hline $\mathrm{O} 7-\mathrm{Zr} 1-\mathrm{O} 3$ & $135.18(3)$ & N2-C6-H6B & 110.2 \\
\hline O5-Zr1-O3 & $71.81(4)$ & C5-C6-H6B & 110.2 \\
\hline O1-Zr1-O3 & $71.32(4)$ & $\mathrm{H} 6 \mathrm{~A}-\mathrm{C} 6-\mathrm{H} 6 \mathrm{~B}$ & 108.5 \\
\hline O7-Zr1-N1 & 81.77 (3) & N2-C7-C8 & $109.67(10)$ \\
\hline O5-Zr1-N1 & 135.27 (4) & N2-C7-H7A & 109.7 \\
\hline O1-Zr1-N1 & $108.72(4)$ & $\mathrm{C} 8-\mathrm{C} 7-\mathrm{H} 7 \mathrm{~A}$ & 109.7 \\
\hline
\end{tabular}




\begin{tabular}{|c|c|c|c|}
\hline $\mathrm{O} 3-\mathrm{Zr} 1-\mathrm{N} 1$ & $140.14(4)$ & $\mathrm{N} 2-\mathrm{C} 7-\mathrm{H} 7 \mathrm{~B}$ & 109.7 \\
\hline $\mathrm{O} 7-\mathrm{Zr} 1-\mathrm{N} 2$ & $122.32(4)$ & $\mathrm{C} 8-\mathrm{C} 7-\mathrm{H} 7 \mathrm{~B}$ & 109.7 \\
\hline $\mathrm{O} 5-\mathrm{Zr} 1-\mathrm{N} 2$ & $148.70(4)$ & $\mathrm{H} 7 \mathrm{~A}-\mathrm{C} 7-\mathrm{H} 7 \mathrm{~B}$ & 108.2 \\
\hline O1-Zr1-N2 & $67.80(4)$ & $\mathrm{O} 2-\mathrm{C} 8-\mathrm{O} 1$ & $125.52(12)$ \\
\hline O3-Zr1-N2 & 79.32 (4) & $\mathrm{O} 2-\mathrm{C} 8-\mathrm{C} 7$ & $118.76(11)$ \\
\hline $\mathrm{N} 1-\mathrm{Zr} 1-\mathrm{N} 2$ & $65.27(4)$ & $\mathrm{O} 1-\mathrm{C} 8-\mathrm{C} 7$ & $115.72(11)$ \\
\hline O7-Zr1-N3 & 153.34 (3) & N2-C9-C10 & $110.38(10)$ \\
\hline $\mathrm{O} 5-\mathrm{Zr} 1-\mathrm{N} 3$ & $86.43(4)$ & $\mathrm{N} 2-\mathrm{C} 9-\mathrm{H} 9 \mathrm{~A}$ & 109.6 \\
\hline O1-Zr1-N3 & $124.82(3)$ & C10-C9-H9A & 109.6 \\
\hline O3-Zr1-N3 & $66.43(4)$ & N2-C9-H9B & 109.6 \\
\hline N1-Zr1-N3 & $84.43(4)$ & C10-C9-H9B & 109.6 \\
\hline N2-Zr1-N3 & $70.86(4)$ & $\mathrm{H} 9 \mathrm{~A}-\mathrm{C} 9-\mathrm{H} 9 \mathrm{~B}$ & 108.1 \\
\hline O7-Zr1-N4 & $82.49(3)$ & N3-C10-C9 & $112.50(11)$ \\
\hline $\mathrm{O} 5-\mathrm{Zr} 1-\mathrm{N} 4$ & $68.01(4)$ & N3-C10-H10A & 109.1 \\
\hline $\mathrm{O} 1-\mathrm{Zr} 1-\mathrm{N} 4$ & $163.82(4)$ & $\mathrm{C} 9-\mathrm{C} 10-\mathrm{H} 10 \mathrm{~A}$ & 109.1 \\
\hline $\mathrm{O} 3-\mathrm{Zr} 1-\mathrm{N} 4$ & $122.15(4)$ & $\mathrm{N} 3-\mathrm{C} 10-\mathrm{H} 10 \mathrm{~B}$ & 109.1 \\
\hline $\mathrm{N} 1-\mathrm{Zr} 1-\mathrm{N} 4$ & $67.59(4)$ & $\mathrm{C} 9-\mathrm{C} 10-\mathrm{H} 10 \mathrm{~B}$ & 109.1 \\
\hline $\mathrm{N} 2-\mathrm{Zr} 1-\mathrm{N} 4$ & $120.96(4)$ & $\mathrm{H} 10 \mathrm{~A}-\mathrm{C} 10-\mathrm{H} 10 \mathrm{~B}$ & 107.8 \\
\hline N3-Zr1-N4 & $71.17(4)$ & N3-C11-C12 & $107.94(10)$ \\
\hline C8-01-Zr1 & $120.30(8)$ & N3-C11-H11A & 110.1 \\
\hline C12-O3-Zr1 & $123.71(8)$ & C12-C11-H11A & 110.1 \\
\hline $\mathrm{C} 16-\mathrm{O} 5-\mathrm{Zr} 1$ & $126.62(8)$ & N3-C11-H11B & 110.1 \\
\hline $\mathrm{Zr} 1-\mathrm{O} 7-\mathrm{Zr} 1^{\mathrm{i}}$ & $167.32(7)$ & C12-C11-H11B & 110.1 \\
\hline $\mathrm{C} 1-\mathrm{N} 1-\mathrm{C} 5$ & $118.36(11)$ & $\mathrm{H} 11 \mathrm{~A}-\mathrm{C} 11-\mathrm{H} 11 \mathrm{~B}$ & 108.4 \\
\hline $\mathrm{C} 1-\mathrm{N} 1-\mathrm{Zr} 1$ & $122.72(8)$ & $\mathrm{O} 4-\mathrm{C} 12-\mathrm{O} 3$ & $124.23(13)$ \\
\hline C5-N1-Zr1 & $118.53(8)$ & $\mathrm{O} 4-\mathrm{C} 12-\mathrm{C} 11$ & $120.54(12)$ \\
\hline C7-N2-C6 & $108.98(10)$ & $\mathrm{O} 3-\mathrm{C} 12-\mathrm{C} 11$ & $115.20(11)$ \\
\hline C7-N2-C9 & $112.25(10)$ & N3-C13-C14 & $110.28(10)$ \\
\hline $\mathrm{C} 6-\mathrm{N} 2-\mathrm{C} 9$ & $109.10(10)$ & $\mathrm{N} 3-\mathrm{C} 13-\mathrm{H} 13 \mathrm{~A}$ & 109.6 \\
\hline
\end{tabular}




\begin{tabular}{|c|c|c|c|}
\hline C7-N2-Zr1 & $107.05(7)$ & $\mathrm{C} 14-\mathrm{C} 13-\mathrm{H} 13 \mathrm{~A}$ & 109.6 \\
\hline C6-N2-Zr1 & $105.70(7)$ & N3-C13-H13B & 109.6 \\
\hline C9-N2-Zr1 & $113.48(7)$ & $\mathrm{C} 14-\mathrm{C} 13-\mathrm{H} 13 \mathrm{~B}$ & 109.6 \\
\hline C13-N3-C11 & $112.01(10)$ & $\mathrm{H} 13 \mathrm{~A}-\mathrm{C} 13-\mathrm{H} 13 \mathrm{~B}$ & 108.1 \\
\hline C13-N3-C10 & $110.36(10)$ & $\mathrm{N} 4-\mathrm{C} 14-\mathrm{C} 13$ & $112.29(10)$ \\
\hline $\mathrm{C} 11-\mathrm{N} 3-\mathrm{C} 10$ & $105.69(10)$ & $\mathrm{N} 4-\mathrm{C} 14-\mathrm{H} 14 \mathrm{~A}$ & 109.1 \\
\hline C13-N3-Zr1 & $110.83(8)$ & $\mathrm{C} 13-\mathrm{C} 14-\mathrm{H} 14 \mathrm{~A}$ & 109.1 \\
\hline C11-N3-Zr1 & $105.52(8)$ & N4-C14-H14B & 109.1 \\
\hline C10-N3-Zr1 & $112.24(8)$ & $\mathrm{C} 13-\mathrm{C} 14-\mathrm{H} 14 \mathrm{~B}$ & 109.1 \\
\hline $\mathrm{C} 15-\mathrm{N} 4-\mathrm{C} 17$ & $109.13(10)$ & $\mathrm{H} 14 \mathrm{~A}-\mathrm{C} 14-\mathrm{H} 14 \mathrm{~B}$ & 107.9 \\
\hline C15-N4-C14 & $108.08(10)$ & $\mathrm{N} 4-\mathrm{C} 15-\mathrm{C} 16$ & $109.33(10)$ \\
\hline C17-N4-C14 & $110.76(10)$ & $\mathrm{N} 4-\mathrm{C} 15-\mathrm{H} 15 \mathrm{~A}$ & 109.8 \\
\hline $\mathrm{C} 15-\mathrm{N} 4-\mathrm{Zr} 1$ & $105.69(7)$ & $\mathrm{C} 16-\mathrm{C} 15-\mathrm{H} 15 \mathrm{~A}$ & 109.8 \\
\hline C17-N4-Zr1 & $111.62(7)$ & N4-C15-H15B & 109.8 \\
\hline C14-N4-Zr1 & $111.36(7)$ & $\mathrm{C} 16-\mathrm{C} 15-\mathrm{H} 15 \mathrm{~B}$ & 109.8 \\
\hline $\mathrm{N} 1-\mathrm{C} 1-\mathrm{C} 2$ & $122.17(12)$ & H15A-C15-H15B & 108.3 \\
\hline $\mathrm{N} 1-\mathrm{C} 1-\mathrm{C} 17$ & $116.40(11)$ & $\mathrm{O} 6-\mathrm{C} 16-\mathrm{O} 5$ & $123.51(13)$ \\
\hline $\mathrm{C} 2-\mathrm{C} 1-\mathrm{C} 17$ & $121.18(12)$ & $\mathrm{O} 6-\mathrm{C} 16-\mathrm{C} 15$ & $121.59(12)$ \\
\hline $\mathrm{C} 3-\mathrm{C} 2-\mathrm{C} 1$ & $118.77(13)$ & $\mathrm{O} 5-\mathrm{C} 16-\mathrm{C} 15$ & $114.88(11)$ \\
\hline $\mathrm{C} 3-\mathrm{C} 2-\mathrm{H} 2$ & 120.6 & $\mathrm{~N} 4-\mathrm{C} 17-\mathrm{C} 1$ & $112.42(10)$ \\
\hline $\mathrm{C} 1-\mathrm{C} 2-\mathrm{H} 2$ & 120.6 & $\mathrm{~N} 4-\mathrm{C} 17-\mathrm{H} 17 \mathrm{~A}$ & 109.1 \\
\hline C2-C3-C4 & $119.41(13)$ & $\mathrm{C} 1-\mathrm{C} 17-\mathrm{H} 17 \mathrm{~A}$ & 109.1 \\
\hline $\mathrm{C} 2-\mathrm{C} 3-\mathrm{H} 3$ & 120.3 & N4-C17-H17B & 109.1 \\
\hline $\mathrm{C} 4-\mathrm{C} 3-\mathrm{H} 3$ & 120.3 & $\mathrm{C} 1-\mathrm{C} 17-\mathrm{H} 17 \mathrm{~B}$ & 109.1 \\
\hline $\mathrm{C} 5-\mathrm{C} 4-\mathrm{C} 3$ & $118.58(13)$ & $\mathrm{H} 17 \mathrm{~A}-\mathrm{C} 17-\mathrm{H} 17 \mathrm{~B}$ & 107.9 \\
\hline $\mathrm{C} 5-\mathrm{C} 4-\mathrm{H} 4$ & 120.7 & $\mathrm{H} 81-\mathrm{O} 8-\mathrm{H} 82$ & $102.1(17)$ \\
\hline $\mathrm{C} 3-\mathrm{C} 4-\mathrm{H} 4$ & 120.7 & H91-O9-H92 & $107.4(17)$ \\
\hline $\mathrm{N} 1-\mathrm{C} 5-\mathrm{C} 4$ & $122.43(13)$ & $\mathrm{H} 101-\mathrm{O} 10-\mathrm{H} 102$ & $104.9(18)$ \\
\hline $\mathrm{N} 1-\mathrm{C} 5-\mathrm{C} 6$ & $114.38(11)$ & $\mathrm{H} 111-\mathrm{O} 11-\mathrm{H} 112$ & $104.7(17)$ \\
\hline
\end{tabular}




\begin{tabular}{|c|c|c|c|}
\hline C4-C5-C6 & $123.15(12)$ & $\mathrm{C} 11-\mathrm{N} 3-\mathrm{C} 10-\mathrm{C} 9$ & $150.29(11)$ \\
\hline $\mathrm{C} 5-\mathrm{N} 1-\mathrm{C} 1-\mathrm{C} 2$ & $-2.78(19)$ & $\mathrm{Zr} 1-\mathrm{N} 3-\mathrm{C} 10-\mathrm{C} 9$ & $35.76(13)$ \\
\hline $\mathrm{Zr} 1-\mathrm{N} 1-\mathrm{C} 1-\mathrm{C} 2$ & $-175.53(10)$ & $\mathrm{N} 2-\mathrm{C} 9-\mathrm{C} 10-\mathrm{N} 3$ & $-51.90(14)$ \\
\hline $\mathrm{C} 5-\mathrm{N} 1-\mathrm{C} 1-\mathrm{C} 17$ & $171.55(11)$ & $\mathrm{C} 13-\mathrm{N} 3-\mathrm{C} 11-\mathrm{C} 12$ & $165.15(11)$ \\
\hline $\mathrm{Zr} 1-\mathrm{N} 1-\mathrm{C} 1-\mathrm{C} 17$ & $-1.20(16)$ & $\mathrm{C} 10-\mathrm{N} 3-\mathrm{C} 11-\mathrm{C} 12$ & $-74.62(13)$ \\
\hline $\mathrm{N} 1-\mathrm{C} 1-\mathrm{C} 2-\mathrm{C} 3$ & $5.4(2)$ & $\mathrm{Zr} 1-\mathrm{N} 3-\mathrm{C} 11-\mathrm{C} 12$ & $44.47(11)$ \\
\hline $\mathrm{C} 17-\mathrm{C} 1-\mathrm{C} 2-\mathrm{C} 3$ & $-168.69(13)$ & $\mathrm{Zr} 1-\mathrm{O} 3-\mathrm{C} 12-\mathrm{O} 4$ & $167.64(12)$ \\
\hline $\mathrm{C} 1-\mathrm{C} 2-\mathrm{C} 3-\mathrm{C} 4$ & $-3.0(2)$ & $\mathrm{Zr} 1-\mathrm{O} 3-\mathrm{C} 12-\mathrm{C} 11$ & $-14.25(16)$ \\
\hline C2-C3-C4-C5 & $-1.6(2)$ & N3-C11-C12-O4 & $153.59(13)$ \\
\hline $\mathrm{C} 1-\mathrm{N} 1-\mathrm{C} 5-\mathrm{C} 4$ & $-2.18(19)$ & N3-C11-C12-O3 & $-24.60(16)$ \\
\hline Zr1-N1-C5-C4 & $170.88(10)$ & C11-N3-C13-C14 & $-71.27(13)$ \\
\hline $\mathrm{C} 1-\mathrm{N} 1-\mathrm{C} 5-\mathrm{C} 6$ & $175.56(12)$ & C10-N3-C13-C14 & $171.26(10)$ \\
\hline Zr1-N1-C5-C6 & $-11.38(15)$ & Zr1-N3-C13-C14 & 46.29 (12) \\
\hline C3-C4-C5-N1 & $4.4(2)$ & C15-N4-C14-C13 & $152.02(11)$ \\
\hline C3-C4-C5-C6 & $-173.17(13)$ & C17-N4-C14-C13 & $-88.48(13)$ \\
\hline C7-N2-C6-C5 & $169.20(10)$ & $\mathrm{Zr} 1-\mathrm{N} 4-\mathrm{C} 14-\mathrm{C} 13$ & $36.36(12)$ \\
\hline C9-N2-C6-C5 & $-67.92(13)$ & $\mathrm{N} 3-\mathrm{C} 13-\mathrm{C} 14-\mathrm{N} 4$ & $-56.36(14)$ \\
\hline $\mathrm{Zr} 1-\mathrm{N} 2-\mathrm{C} 6-\mathrm{C} 5$ & $54.44(11)$ & C17-N4-C15-C16 & $160.02(10)$ \\
\hline $\mathrm{N} 1-\mathrm{C} 5-\mathrm{C} 6-\mathrm{N} 2$ & $-30.48(16)$ & C14-N4-C15-C16 & $-79.45(12)$ \\
\hline $\mathrm{C} 4-\mathrm{C} 5-\mathrm{C} 6-\mathrm{N} 2$ & $147.25(13)$ & Zr1-N4-C15-C16 & 39.86 (11) \\
\hline $\mathrm{C} 6-\mathrm{N} 2-\mathrm{C} 7-\mathrm{C} 8$ & $-82.07(12)$ & $\mathrm{Zr} 1-\mathrm{O} 5-\mathrm{C} 16-\mathrm{O} 6$ & $172.59(10)$ \\
\hline $\mathrm{C} 9-\mathrm{N} 2-\mathrm{C} 7-\mathrm{C} 8$ & $156.95(10)$ & $\mathrm{Zr} 1-\mathrm{O} 5-\mathrm{C} 16-\mathrm{C} 15$ & $-5.77(16)$ \\
\hline $\mathrm{Zr} 1-\mathrm{N} 2-\mathrm{C} 7-\mathrm{C} 8$ & $31.81(11)$ & $\mathrm{N} 4-\mathrm{C} 15-\mathrm{C} 16-\mathrm{O} 6$ & $154.58(12)$ \\
\hline $\mathrm{Zr} 1-\mathrm{O} 1-\mathrm{C} 8-\mathrm{O} 2$ & $147.14(11)$ & $\mathrm{N} 4-\mathrm{C} 15-\mathrm{C} 16-\mathrm{O} 5$ & $-27.02(15)$ \\
\hline $\mathrm{Zr} 1-\mathrm{O} 1-\mathrm{C} 8-\mathrm{C} 7$ & $-32.89(14)$ & $\mathrm{C} 15-\mathrm{N} 4-\mathrm{C} 17-\mathrm{C} 1$ & $-150.19(11)$ \\
\hline N2-C7-C8-O2 & $175.40(11)$ & C14-N4-C17-C1 & $90.95(12)$ \\
\hline N2-C7-C8-O1 & $-4.57(15)$ & $\mathrm{Zr} 1-\mathrm{N} 4-\mathrm{C} 17-\mathrm{C} 1$ & $-33.74(13)$ \\
\hline C7-N2-C9-C10 & $-79.60(13)$ & $\mathrm{N} 1-\mathrm{C} 1-\mathrm{C} 17-\mathrm{N} 4$ & $24.35(16)$ \\
\hline $\mathrm{C} 6-\mathrm{N} 2-\mathrm{C} 9-\mathrm{C} 10$ & $159.49(10)$ & $\mathrm{C} 2-\mathrm{C} 1-\mathrm{C} 17-\mathrm{N} 4$ & $-161.26(12)$ \\
\hline
\end{tabular}




\begin{tabular}{|l|l|l|l|}
\hline Zr1-N2-C9-C10 & $41.93(12)$ & & \\
\hline C13-N3-C10-C9 & $-88.40(13)$ & & \\
\hline
\end{tabular}

Symmetry code: (i) $-x+1, y,-z+1 / 2$.

Table S10. Hydrogen-bond geometry ( $(\AA, \stackrel{\circ}{)})$

\begin{tabular}{|l|l|l|l|l|}
\hline$D-\mathrm{H} \cdots A$ & $D-\mathrm{H}$ & $\mathrm{H} \cdots A$ & $D \cdots A$ & $D-\mathrm{H} \cdots A$ \\
\hline $\mathrm{C} 2-\mathrm{H} 2 \cdots \mathrm{O}^{\mathrm{ii}}$ & 0.95 & 2.59 & $3.5066(18)$ & 163 \\
\hline $\mathrm{C} 4-\mathrm{H} 4 \cdots \mathrm{O} 2^{\mathrm{iii}}$ & 0.95 & 2.50 & $3.2094(17)$ & 132 \\
\hline $\mathrm{C} 6-\mathrm{H} 6 B \cdots \mathrm{O} 6^{\mathrm{iv}}$ & 0.99 & 2.61 & $3.3529(16)$ & 132 \\
\hline $\mathrm{C} 7-\mathrm{H} 7 B \cdots \mathrm{O} 10^{\mathrm{iv}}$ & 0.99 & 2.46 & $3.396(2)$ & 158 \\
\hline $\mathrm{C} 9-\mathrm{H} 9 A \cdots \mathrm{O} 11^{1 i}$ & 0.99 & 2.41 & $3.3316(19)$ & 155 \\
\hline $\mathrm{C} 14-\mathrm{H} 14 B \cdots \mathrm{O} 4^{\mathrm{ii}}$ & 0.99 & 2.63 & $3.3596(18)$ & 131 \\
\hline $\mathrm{C} 15-\mathrm{H} 15 A \cdots \mathrm{O} 5^{\mathrm{i}}$ & 0.99 & 2.56 & $3.4912(18)$ & 158 \\
\hline $\mathrm{C} 15-\mathrm{H} 15 B \cdots \mathrm{O} 2^{\mathrm{ii}}$ & 0.99 & 2.34 & $3.2556(17)$ & 154 \\
\hline $\mathrm{O} 8-\mathrm{H} 81 \cdots \mathrm{O} 2$ & $0.82(1)$ & $2.04(1)$ & $2.8598(16)$ & $171(2)$ \\
\hline $\mathrm{O} 8-\mathrm{H} 82 \cdots \mathrm{O} 11^{\mathrm{iv}}$ & $0.82(1)$ & $1.97(1)$ & $2.7852(18)$ & $171(2)$ \\
\hline $\mathrm{O} 9-\mathrm{H} 91 \cdots \mathrm{O} 4$ & $0.81(1)$ & $1.90(1)$ & $2.7093(16)$ & $176(2)$ \\
\hline $\mathrm{O}-\mathrm{H} 92 \cdots \mathrm{O} 8^{\vee}$ & $0.81(1)$ & $1.93(1)$ & $2.7335(18)$ & $175(2)$ \\
\hline $\mathrm{O} 10-\mathrm{H} 101 \cdots \mathrm{O} 6$ & $0.81(1)$ & $1.98(1)$ & $2.7669(18)$ & $164(2)$ \\
\hline $\mathrm{O} 10-\mathrm{H} 102 \cdots \mathrm{O} 9^{v i}$ & $0.82(1)$ & $2.02(1)$ & $2.8285(19)$ & $169(2)$ \\
\hline $\mathrm{O} 11-\mathrm{H} 111 \cdots \mathrm{O} 10$ & $0.82(1)$ & $1.92(1)$ & $2.7262(18)$ & $170(2)$ \\
\hline $\mathrm{O} 11-\mathrm{H} 112 \cdots \mathrm{O} 9$ & $0.81(1)$ & $2.09(1)$ & $2.8574(18)$ & $158(2)$ \\
\hline
\end{tabular}

Symmetry codes: (i) $-x+1, y,-z+1 / 2$; (ii) $x,-y+1, z+1 / 2$; (iii) $x,-y, z+1 / 2$; (iv) $x, y-1, z$; (v) $-x+1 / 2, y+1 / 2,-z-1 / 2$; (vi) $-x+1 / 2,-y+3 / 2,-z$ 
Least-squares planes ( $x, y, z$ in crystal coordinates) and deviations from them (* indicates atom used to define plane)

$21.3519(0.0087) x+3.2726(0.0041) y-12.1847(0.0048) z=6.5766(0.0038)$

* $-0.3241(0.0006) \mathrm{N} 1$

* $0.2939(0.0006) \mathrm{N} 2$

* $-0.2499(0.0005) \mathrm{N} 3$

* $0.2802(0.0005) \mathrm{N} 4$

$1.5091(0.0006) \mathrm{Zr} 1$

$2.8172(0.0013) 01$

$2.1775(0.0015) \mathrm{O3}$

$2.4921(0.0014) 05$

$2.4212(0.0011) 07$

Rms deviation of fitted atoms $=0.2883$

$19.8900(0.0061) x+4.1453(0.0038) y-12.2662(0.0037) z=8.7824(0.0028)$

Angle to previous plane (with approximate esd) $=5.852(0.053)$

* $0.1912(0.0005) 01$

* $-0.1994(0.0005) 03$

* $0.1793(0.0004) 05$

* $-0.1711(0.0004) 07$

$-0.9813(0.0004) \mathrm{Zr} 1$

$-2.9373(0.0012) \mathrm{N} 1$

$-2.3114(0.0014) \mathrm{N} 2$

$-2.5779(0.0014)$ N3

$-2.0929(0.0014) \mathrm{N} 4$

Rms deviation of fitted atoms $=0.1855$ 


\section{Crystal Structure Analysis of $\mathrm{Zr}-\mathrm{NOTA},\left[\mathrm{Zr}\left(\mathrm{C}_{12} \mathrm{H}_{18} \mathrm{~N}_{3} \mathrm{O}_{6}\right)(\mathrm{OH})\right]_{2}-6 \mathrm{H}_{2} \mathrm{O}$}

\section{Crystallographic Experimental Details:}

Data Collection and Structure Solution. A clear colorless plate-like specimen of $\left[\mathrm{Zr}\left(\mathrm{C}_{12} \mathrm{H}_{18} \mathrm{~N}_{3} \mathrm{O}_{6}\right)(\mathrm{OH})\right]_{2}-6$ $\mathrm{H}_{2} \mathrm{O}$, approximate dimensions $0.080 \mathrm{~mm} \times 0.300 \mathrm{~mm} \times 0.460 \mathrm{~mm}$, was used for the X-ray crystallographic analysis. The X-ray intensity data were measured on a Bruker APEX CCD system equipped with a graphite monochromator and a Mo $K_{\alpha}$ sealed $x$-ray tube $(\lambda=0.71073 \AA)$. X-rays were provided by a fine-focus sealed $x$ ray tube operated at $50 \mathrm{kV}$ and $30 \mathrm{~mA}$.

The total exposure time was 18.73 hours. The frames were integrated with the Bruker SAINT Software ${ }^{1}$ package using a narrow-frame algorithm. The integration of the data using a monoclinic unit cell yielded a total of 31714 reflections to a maximum $\theta$ angle of $30.50^{\circ}(0.70 \AA$ resolution), of which 5078 were independent (average redundancy 6.245 , completeness $=99.8 \%, R_{\text {int }}=3.13 \%, R_{\text {sig }}=1.83 \%$ ) and $4778(94.09 \%)$ were greater than $2 \sigma\left(F^{2}\right)$. The final cell constants of $a=11.7960$ (7) $\AA, b=10.9411$ (6) $\AA, c=14.1815$ (8) $\AA, \beta=114.2100$ $(10)^{\circ}$, volume $=1669.31(16) \AA^{3}$, are based upon the refinement of the $X Y Z$-centroids of 9847 reflections above $20 \sigma(\mathrm{I})$ with $7.32^{\circ}<2 \theta<62.98^{\circ}$. Data were corrected for absorption effects using the multi-scan method $(\mathrm{SADABS})^{2}$. The ratio of minimum to maximum apparent transmission was 0.909 . The calculated minimum and maximum transmission coefficients (based on crystal size) are 0.7330 and 0.9450 .

The structure was solved and refined using the Bruker SHELXTL Software Package ${ }^{3}$, using the space group $\mathrm{P} 2{ }_{1} / \mathrm{n}$, with $\mathrm{Z}=2$ for the formula unit, $\mathrm{C}_{24} \mathrm{H}_{50} \mathrm{~N}_{6} \mathrm{O}_{20} \mathrm{Zr}_{2}$. The final model includes 6 full-occupancy water molecules per dimeric unit. The arrangement of three $\mathrm{CH} 2$ groups about nitrogen atom $\mathrm{N} 3$ shows a slight rotational disorder (93/7\%) about the Zr-N3 bond. The carbon atoms for the major orientation are designated by carbons C8, C10 and $\mathrm{C} 11$ and their associated hydrogen atoms. The carbon atoms for the minor orientation are designated by carbons $\mathrm{C}^{\prime}, \mathrm{C} 10^{\prime}$ and $\mathrm{C} 11$ ' and their associated hydrogens. The final structural model incorporated isotropic thermal parameters for the carbon atoms of the minor orientation and for all included hydrogen atoms. The hydrogen atoms of the "NOTA" ligand were included in the structural model as fixed atoms (using idealized sp³ hybridized geometry and $\mathrm{C}-\mathrm{H}$ bond lengths of $0.99 \AA$ ) "riding" on their respective carbon atoms. The isotropic thermal parameters for all "NOTA" hydrogen atoms were fixed at values 1.2 times the equivalent isotropic thermal parameter of the carbon atom to which they are covalently bonded. The isotropic thermal parameters of the 
water hydrogen atoms were allowed to refine independently. The final anisotropic full-matrix least-squares refinement on $F^{2}$ with 276 variables converged at $R_{1}=1.92 \%$, for the observed data and $w R_{2}=5.18 \%$ for all data. The goodness-of-fit was 1.030 . The largest peak in the final difference electron density synthesis was 0.521 $\mathrm{e}^{-} / \AA^{3}$ and the largest hole was $-0.240 \mathrm{e} / \AA^{3}$ with an RMS deviation of $0.060 \mathrm{e}^{-} / \AA^{3}$. On the basis of the final model, the calculated density was $1.841 \mathrm{~g} / \mathrm{cm}^{3}$ and $\mathrm{F}(000), 952 \mathrm{e}^{-}$.

Refinement Details. Crystal data, data collection and structure refinement details are summarized in tables below.

Computing details. Data collection: Bruker APEX3 v2016.1-0; cell refinement: Bruker APEX3 v2016.1-0; data reduction: Bruker APEX2 v2014.11-0; program(s) used to solve structure: SHELXS97 (Sheldrick 2008); program(s) used to refine structure: SHELXL2014 (Sheldrick, 2014); molecular graphics: Bruker APEX2 v2014.11-0; software used to prepare material for publication: Bruker APEX2 v2014.11-01-6. 


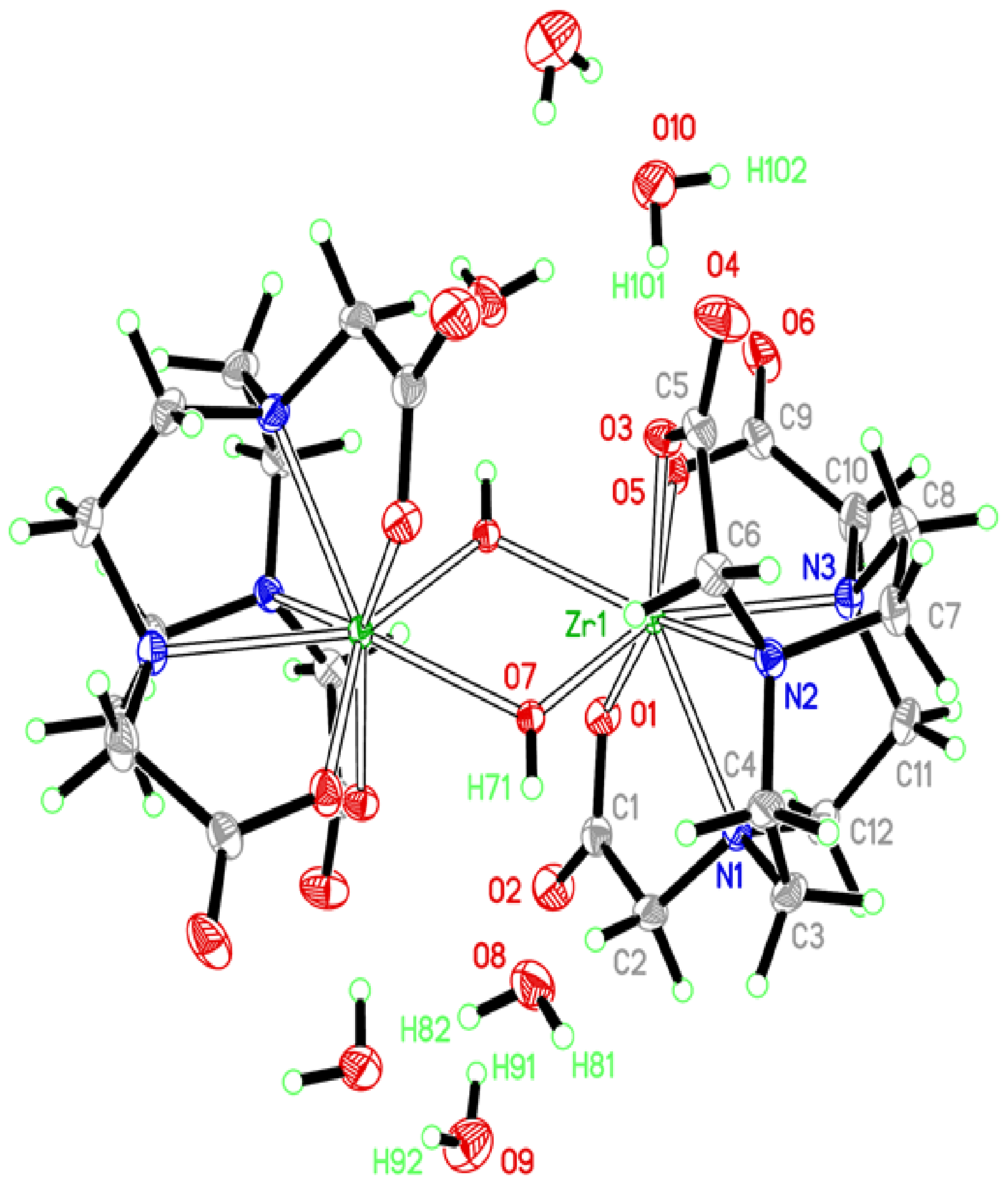

Figure S35. $50 \%$ probability plot for the dimeric unit of $\left[\mathrm{Zr}\left(\mathrm{C}_{12} \mathrm{H}_{18} \mathrm{~N}_{3} \mathrm{O}_{6}\right)(\mathrm{OH})\right]_{2}-6 \mathrm{H}_{2} \mathrm{O}$ with disordered atoms C8', C10' and C11' omitted for clarity. The two halves of the dimer are related by the crystallographic inversion center at $1 \frac{2}{2}, 1 / 2,0$ in the unit cell. Only the non-hydrogen atoms of the asymmetric unit are labeled. 


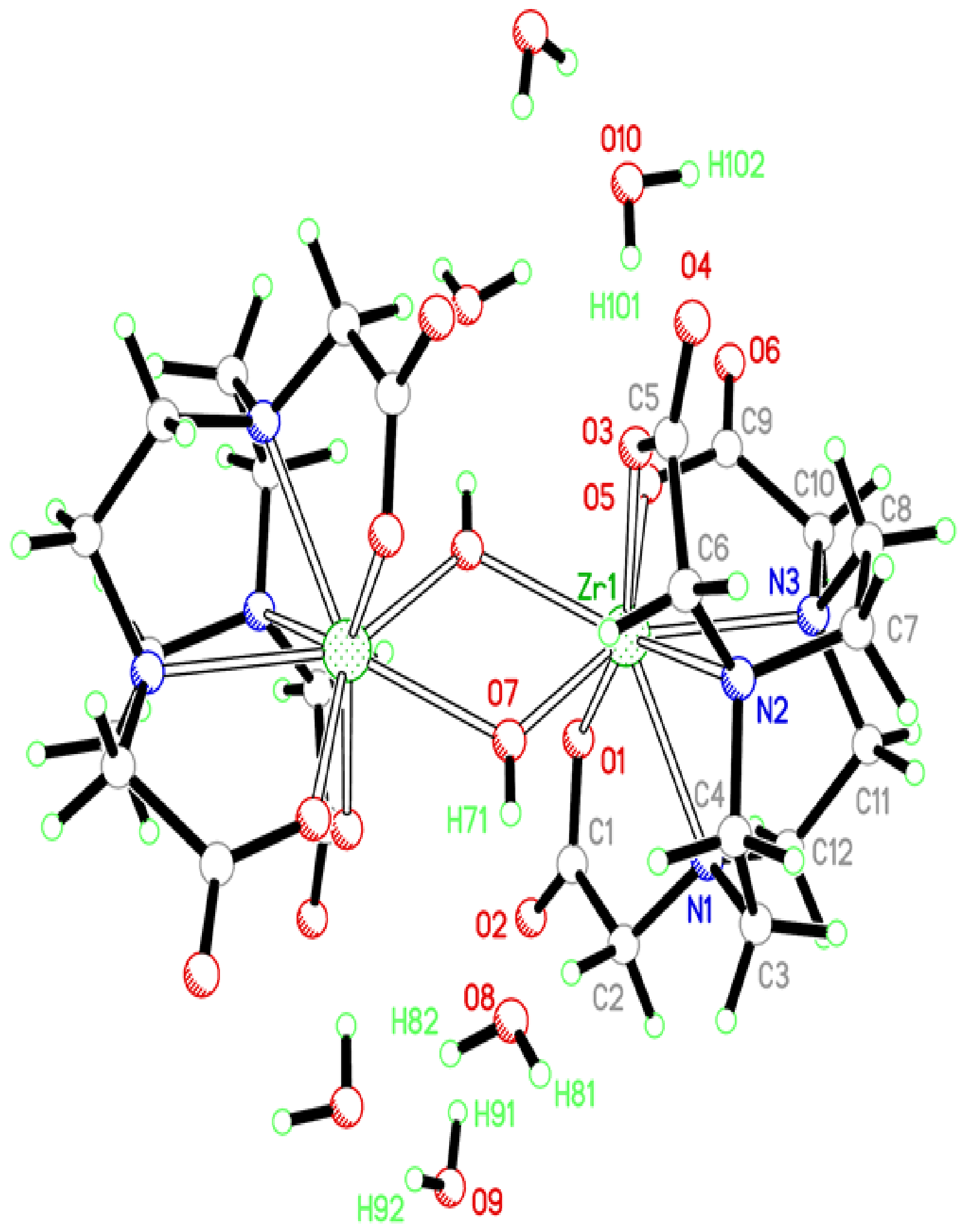

Figure S36. Plot of $\left[\mathrm{Zr}\left(\mathrm{C}_{12} \mathrm{H}_{18} \mathrm{~N}_{3} \mathrm{O}_{6}\right)(\mathrm{OH})\right]_{2}-6 \mathrm{H}_{2} \mathrm{O}$ with disordered atoms C8', C10' and C11' omitted for clarity. All atoms represented by arbitrary-sized spheres. 


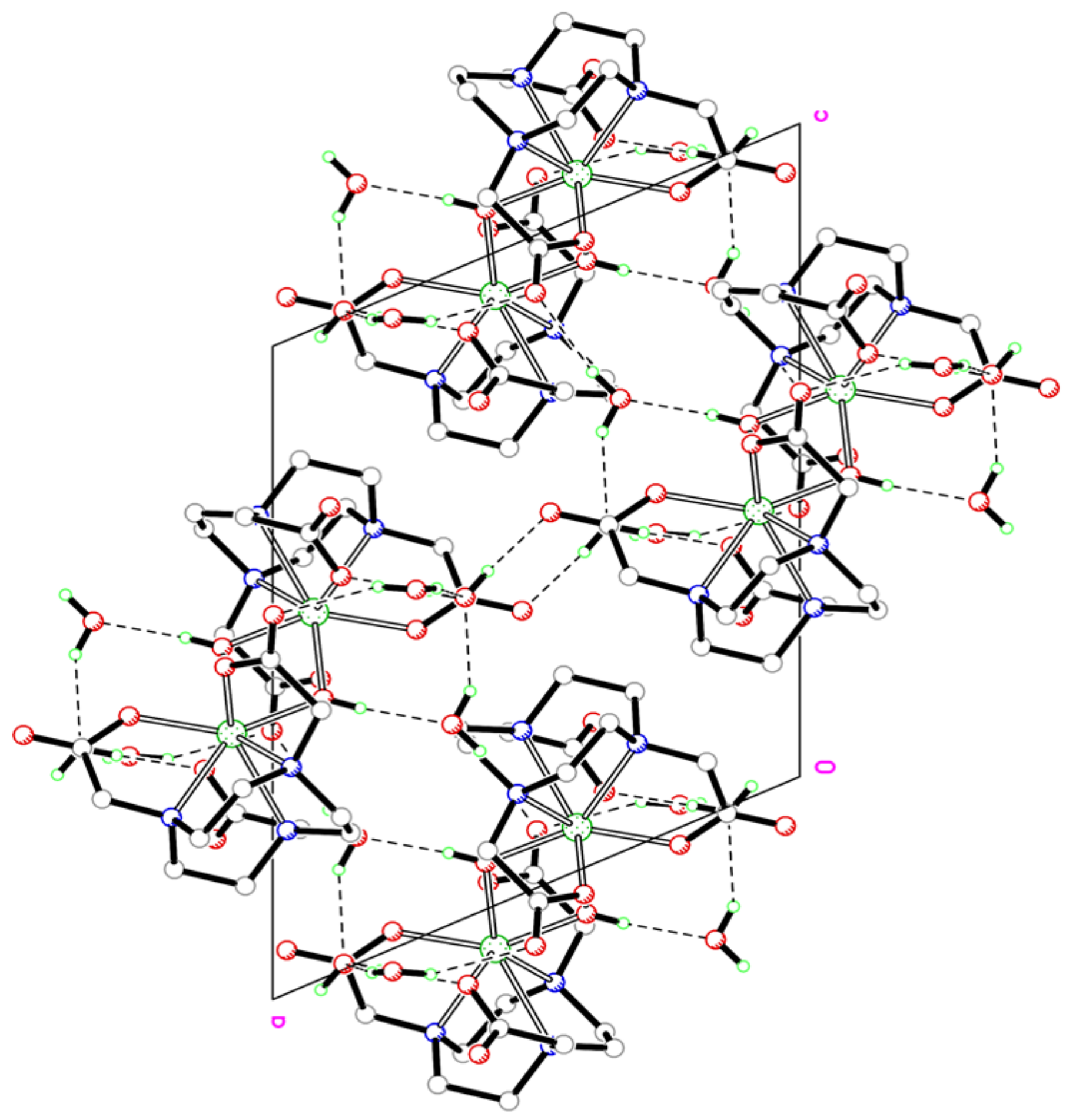

Figure S37. Packing plot of the solid-state structure of $\left[\mathrm{Zr}\left(\mathrm{C}_{12} \mathrm{H}_{18} \mathrm{~N}_{3} \mathrm{O}_{6}\right)(\mathrm{OH})\right]_{2}-6 \mathrm{H}_{2} \mathrm{O}$ viewed in projection down the $b$ axis of the unit cell showing the major orientation (93\%). All atoms are represented by dummysized spheres and ligand hydrogen atoms have been omitted for clarity. Hydrogen-bonding interactions are represented by dashed-solid bonds. 


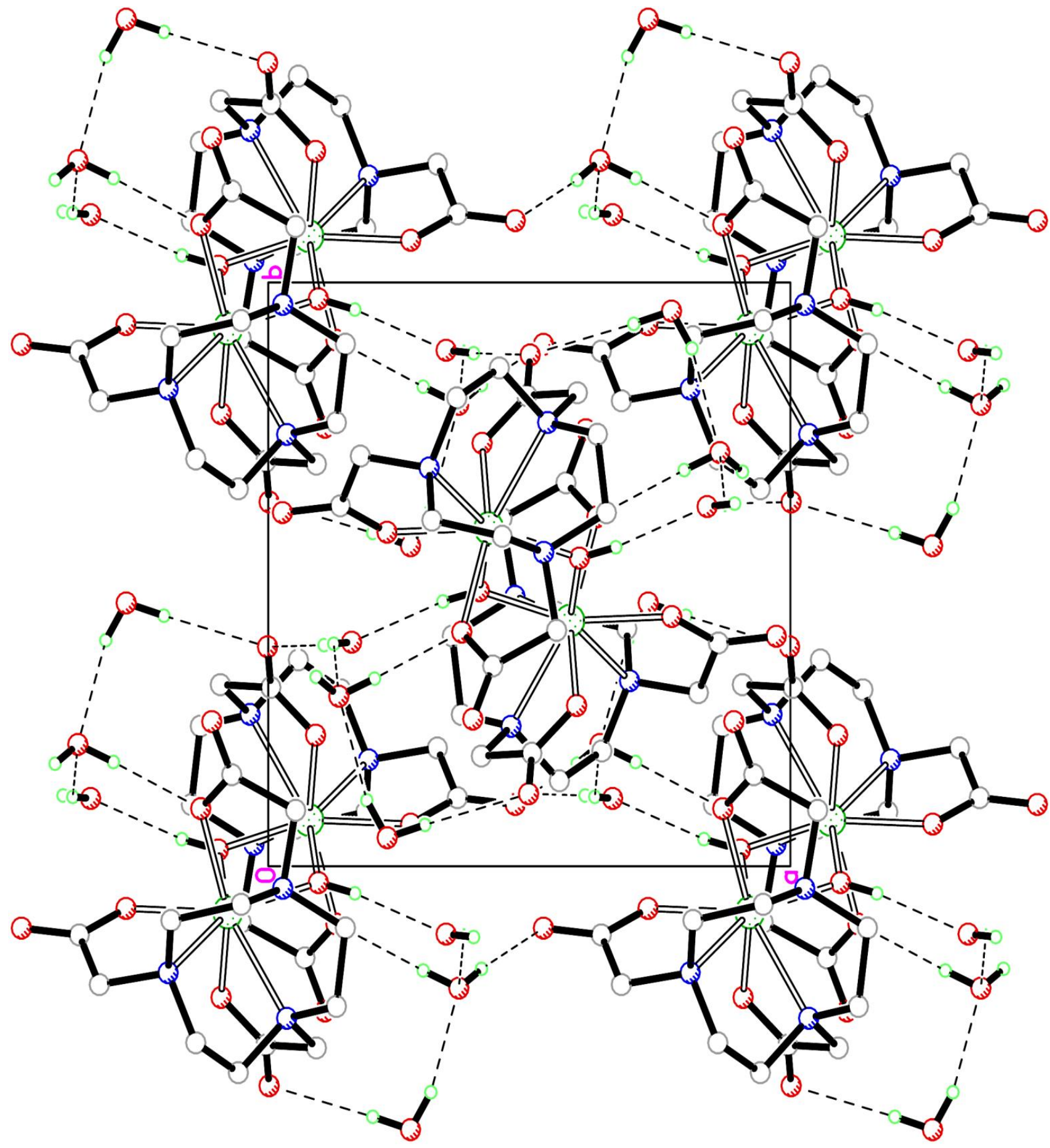

Figure S38. Packing plot of the solid-state structure of $\left[\mathrm{Zr}\left(\mathrm{C}_{12} \mathrm{H}_{18} \mathrm{~N}_{3} \mathrm{O}_{6}\right)(\mathrm{OH})\right]_{2}-6 \mathrm{H}_{2} \mathrm{O}$ viewed in projection down the $c$ axis of the unit cell showing the major orientation (93\%). All atoms are represented by dummysized spheres and ligand hydrogen atoms have been omitted for clarity. Hydrogen-bonding interactions are represented by dashed-solid bonds. 


\section{R}

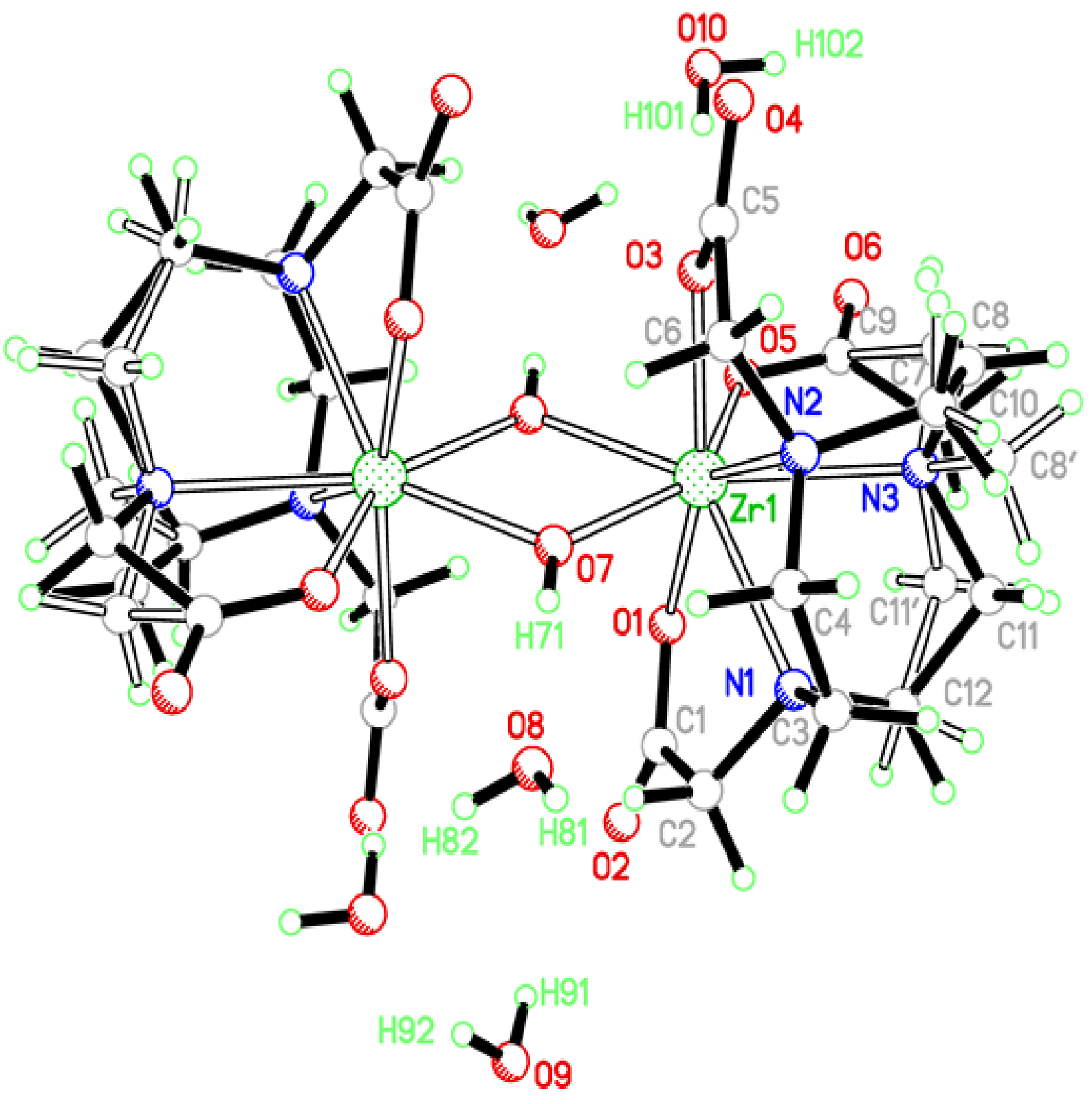

Figure S39. Plot of $\left[\mathrm{Zr}\left(\mathrm{C}_{12} \mathrm{H}_{18} \mathrm{~N}_{3} \mathrm{O}_{6}\right)(\mathrm{OH})\right]_{2}-6 \mathrm{H}_{2} \mathrm{O}$ showing the disordered arrangement of carbon atoms about the Zr1-N3 bond with the major (93\%) orientation designated by carbon atoms C8, C10 and C11 and the minor (7\%) orientation designated by carbon atoms C8', C10' and C11'. All atoms are represented by arbitrary-sized spheres. 


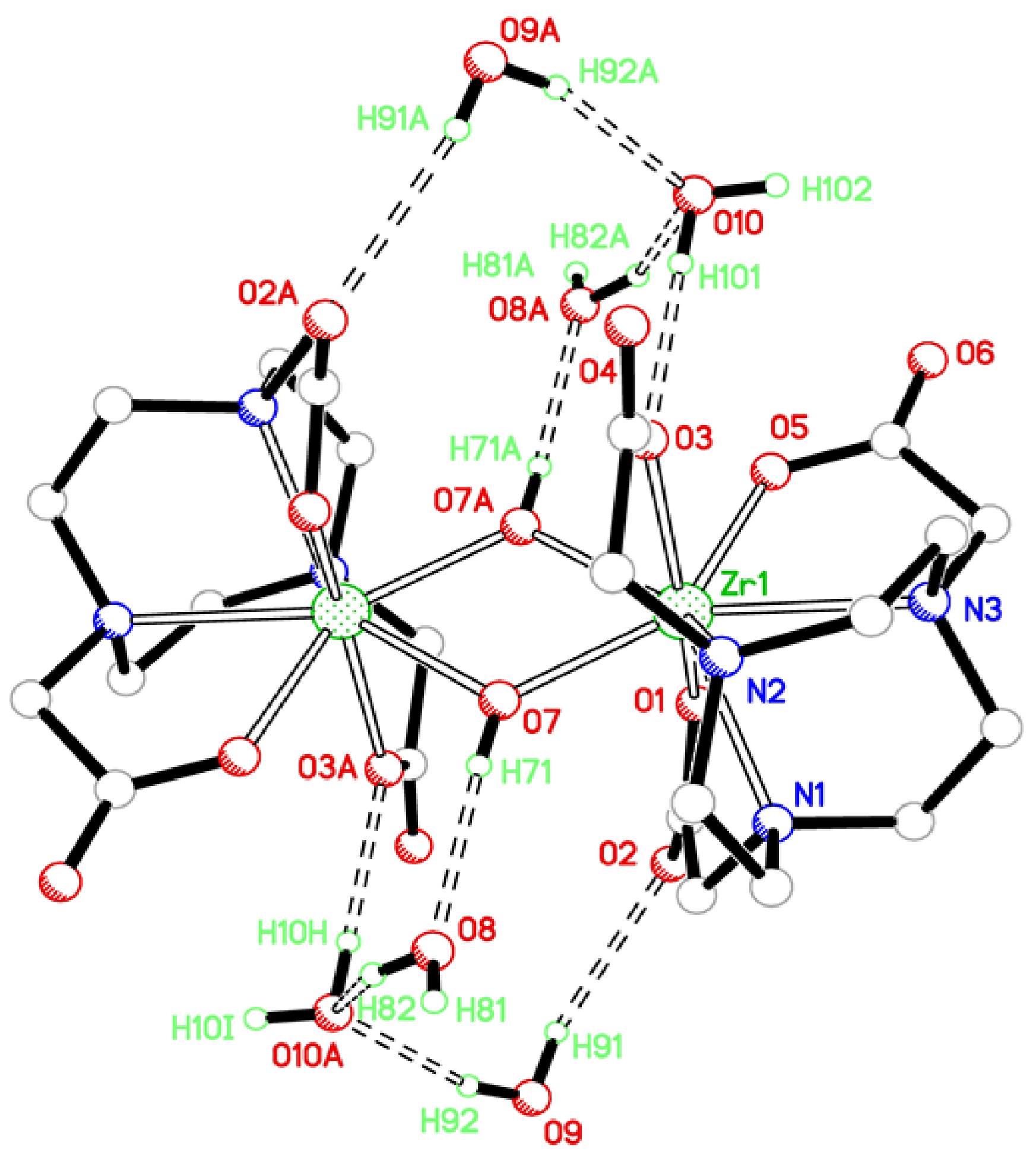

Figure S40. Plot of $\left[\mathrm{Zr}\left(\mathrm{C}_{12} \mathrm{H}_{18} \mathrm{~N}_{3} \mathrm{O}_{6}\right)(\mathrm{OH})\right]_{2}-6 \mathrm{H}_{2} \mathrm{O}$ with disordered atoms C8', C10' and C11' omitted for clarity. All atoms represented by arbitrary-sized spheres. Ligand hydrogen atoms have been omitted and hydrogen-bonding interactions involving the bridging $\mathrm{OH}$ groups and lattice water molecules are represented by dashed-open bonds. 


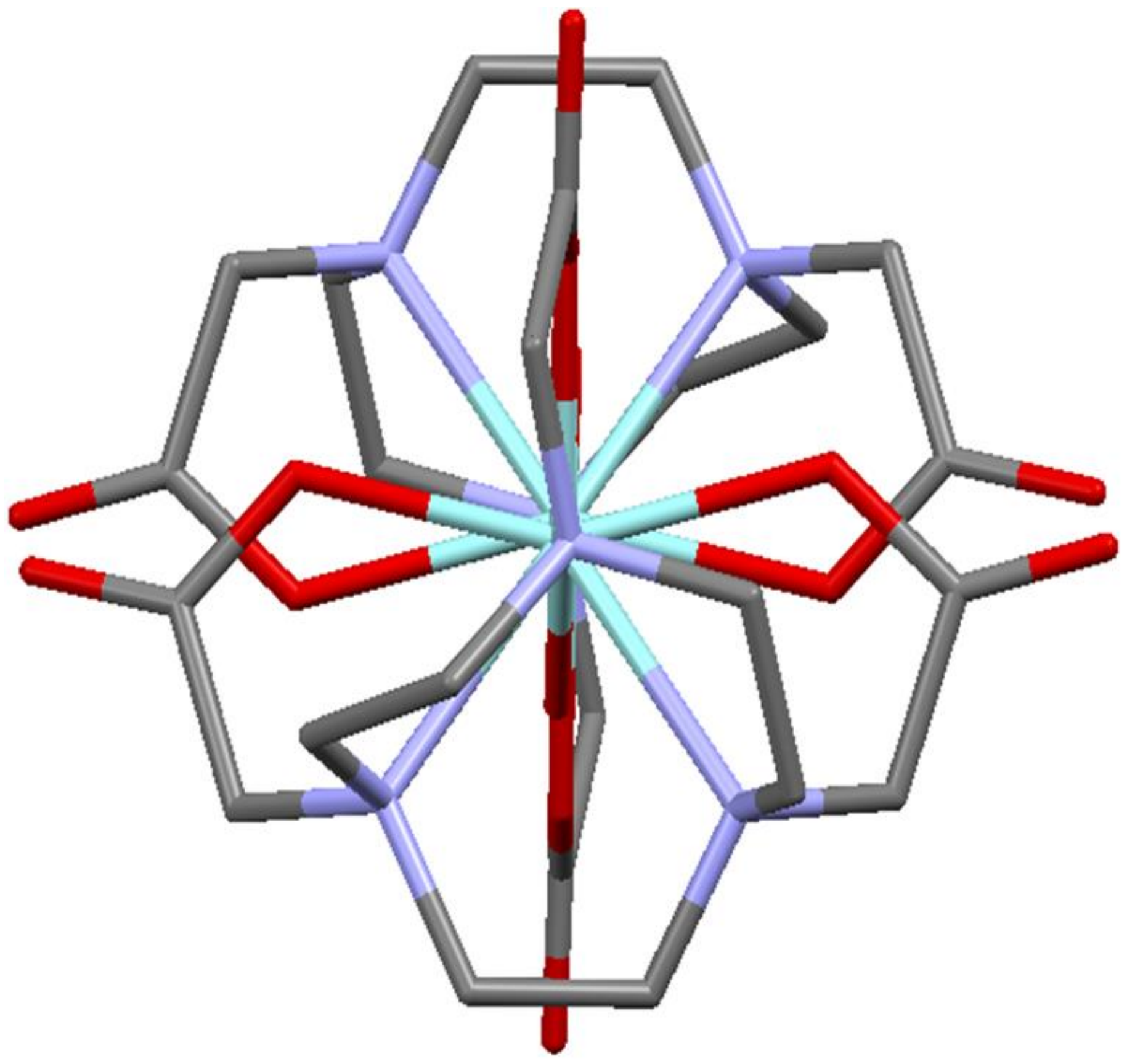

Figure S41. Plot of $\left[\mathrm{Zr}\left(\mathrm{C}_{12} \mathrm{H}_{18} \mathrm{~N}_{3} \mathrm{O}_{6}\right)(\mathrm{OH})\right]_{2}-6 \mathrm{H}_{2} \mathrm{O}$, viewed down the $\mathrm{Zr}$ - $\mathrm{Zr}$ vector, with disordered atoms C8', C10' and C11'. All hydrogen atoms and water molecules have been omitted for clarity. 
Table 11. Crystal structure data collection parameters

\section{Crystal data}

\begin{tabular}{|l|l|}
\hline $\mathrm{C}_{24} \mathrm{H}_{50} \mathrm{~N}_{6} \mathrm{O}_{20} \mathrm{Zr}_{2}$ & $\mathrm{~F}(000)=952$ \\
\hline$M_{r}=925.14$ & $D_{x}=1.841 \mathrm{Mg} \mathrm{m}^{-3}$ \\
\hline Monoclinic, $P 2_{1} / n$ & Mo Ka radiation, $\lambda=0.71073 \AA$ \\
\hline$a=11.7960(7) \AA$ & Cell parameters from 9847 reflections \\
\hline$b=10.9411(6) \AA$ & $\theta=3.7-31.5^{\circ}$ \\
\hline$C=14.1815(8) \AA$ & $\mu=0.72 \mathrm{~mm}^{-1}$ \\
\hline$\beta=114.210(1)^{\circ}$ & $T=193 \mathrm{~K}$ \\
\hline$V=1669.31(16) \AA 3$ & Irregular plate, colorless \\
\hline$Z=2$ & $0.46 \times 0.30 \times 0.08 \mathrm{~mm}$ \\
\hline
\end{tabular}

\section{Data collection}

\begin{tabular}{|l|l|}
\hline Bruker APEX CCD diffractometer & 5078 independent reflections \\
\hline Radiation source: sealed x-ray tube & 4778 reflections with $I>2 \sigma(I)$ \\
\hline Graphite monochromator & $R_{\text {int }}=0.031$ \\
\hline$\phi$ and $\omega$ scans & $\theta_{\max }=30.5^{\circ}, \theta_{\min }=3.5^{\circ}$ \\
\hline $\begin{array}{l}\text { Absorption correction: multi-scan Data were } \\
\text { corrected for scaling and absorption effects using } \\
\text { the multi-scan technique (SADABS). The ratio of } \\
\text { minimum to maximum apparent transmission was } \\
0.909 . \text { The calculated minimum and maximum } \\
\text { transmission coefficients (based on crystal size) are } \\
0.733 \text { and } 0.945 .\end{array}$ & $h=-16 \rightarrow 16$ \\
\hline $\begin{array}{l}T_{\text {min }}=0.678, T_{\max }=0.746 \\
31714 \text { measured reflections }\end{array}$ & $k=-15 \rightarrow 15$ \\
\hline
\end{tabular}




\section{Refinement}

\begin{tabular}{|l|l|}
\hline Refinement on $F^{2}$ & 10 restraints \\
\hline Least-squares matrix: full & Hydrogen site location: mixed \\
\hline$R\left[F^{2}>2 \sigma\left(F^{2}\right)\right]=0.019$ & $\begin{array}{l}\text { H atoms treated by a mixture of independent and } \\
\text { constrained refinement }\end{array}$ \\
\hline$w R\left(F^{2}\right)=0.052$ & $\begin{array}{l}w=1 /\left[\sigma^{2}\left(F_{0}^{2}\right)+(0.0269 P)^{2}+0.7232 P\right] \\
\text { where } P=\left(F_{0}^{2}+2 F_{c}^{2}\right) / 3\end{array}$ \\
\hline$S=1.03$ & $(\Delta / \sigma)_{\max }=0.002$ \\
\hline 5078 reflections & $\Delta\rangle_{\max }=0.52$ e $\AA^{-3}$ \\
\hline 276 parameters & $\Delta\rangle_{\min }=-0.24$ e $\AA^{-3}$ \\
\hline
\end{tabular}

\section{Special details}

Geometry. All esds (except the esd in the dihedral angle between two I.s. planes) are estimated using the full covariance matrix. The cell esds are taken into account individually in the estimation of esds in distances, angles and torsion angles; correlations between esds in cell parameters are only used when they are defined by crystal symmetry. An approximate (isotropic) treatment of cell esds is used for estimating esds involving I.s. planes. 
Table S12. Fractional atomic coordinates and isotropic or equivalent isotropic displacement parameters $\left(\AA^{2}\right)$

\begin{tabular}{|c|c|c|c|c|c|}
\hline & $x$ & $y$ & $z$ & $U_{\text {iso }}{ }^{*} / U_{\text {eq }}$ & Occ. $(<1)$ \\
\hline Zr1 & $0.42226(2)$ & $0.57931(2)$ & 0.06624 (2) & 0.00986 (4) & \\
\hline 01 & 0.40948 (7) & $0.72464(7)$ & $-0.04018(6)$ & $0.01716(15)$ & \\
\hline $\mathrm{O} 2$ & $0.50149(9)$ & $0.87590(9)$ & $-0.08801(7)$ & $0.02681(19)$ & \\
\hline O3 & 0.37057 (7) & $0.40118(7)$ & $0.10355(6)$ & $0.01641(15)$ & \\
\hline O4 & $0.39167(9)$ & $0.25215(9)$ & $0.21731(7)$ & $0.0285(2)$ & \\
\hline O5 & $0.22734(7)$ & $0.57222(8)$ & $-0.02677(6)$ & $0.01836(16)$ & \\
\hline 06 & $0.02701(8)$ & $0.60589(10)$ & $-0.06612(7)$ & $0.02521(19)$ & \\
\hline O7 & $0.59586(7)$ & $0.52797(7)$ & $0.07190(6)$ & $0.01279(14)$ & \\
\hline $\mathrm{H} 71$ & $0.6656(10)$ & $0.5464(17)$ & $0.1103(12)$ & $0.032(5)^{*}$ & \\
\hline N1 & $0.53517(8)$ & 0.76027 (8) & $0.15638(7)$ & $0.01482(16)$ & \\
\hline N2 & 0.52750 (8) & 0.53771 (9) & $0.25042(7)$ & $0.01404(16)$ & \\
\hline N3 & 0.30800 (8) & $0.68228(9)$ & $0.15594(7)$ & $0.01593(17)$ & \\
\hline C1 & 0.49519 (11) & $0.80627(10)$ & $-0.02259(9)$ & $0.0173(2)$ & \\
\hline C2 & $0.59196(10)$ & $0.81112(10)$ & 0.08854 (8) & 0.0179 (2) & \\
\hline $\mathrm{H} 2 \mathrm{~A}$ & 0.6659 & 0.7625 & 0.0958 & $0.021^{*}$ & \\
\hline $\mathrm{H} 2 \mathrm{~B}$ & 0.6185 & 0.8966 & 0.1084 & $0.021^{*}$ & \\
\hline C3 & $0.63025(11)$ & 0.74006 (11) & $0.26511(8)$ & 0.0187 (2) & \\
\hline $\mathrm{H} 3 \mathrm{~A}$ & 0.6042 & 0.7846 & 0.3136 & $0.022^{*}$ & \\
\hline H3B & 0.7109 & 0.7744 & 0.2719 & $0.022^{*}$ & \\
\hline C4 & $0.64753(10)$ & $0.60560(11)$ & 0.29505 (8) & $0.0170(2)$ & \\
\hline $\mathrm{H} 4 \mathrm{~A}$ & 0.7068 & 0.5685 & 0.2702 & $0.020^{*}$ & \\
\hline $\mathrm{H} 4 \mathrm{~B}$ & 0.6834 & 0.5984 & 0.3713 & $0.020^{*}$ & \\
\hline C5 & $0.42989(10)$ & $0.34316(10)$ & $0.19085(8)$ & $0.01681(19)$ & \\
\hline C6 & $0.55222(10)$ & $0.40409(10)$ & 0.25705 (9) & 0.0167 (2) & \\
\hline $\mathrm{H} 6 \mathrm{~A}$ & 0.5821 & 0.3759 & 0.3296 & $0.020^{*}$ & \\
\hline H6B & 0.6161 & 0.3844 & 0.2309 & $0.020^{*}$ & \\
\hline C7 & 0.44795 (11) & $0.56521(11)$ & 0.30816 (9) & $0.0189(2)$ & \\
\hline
\end{tabular}




\begin{tabular}{|c|c|c|c|c|c|}
\hline H7A & 0.4834 & 0.6362 & 0.3541 & $0.023^{*}$ & 0.932 (3) \\
\hline H7B & 0.4502 & 0.4943 & 0.3522 & $0.023^{*}$ & $0.932(3)$ \\
\hline $\mathrm{H} 7 \mathrm{C}$ & 0.3961 & 0.4927 & 0.3042 & $0.023^{*}$ & 0.068 (3) \\
\hline H7D & 0.5031 & 0.5785 & 0.3819 & $0.023^{*}$ & 0.068 (3) \\
\hline C8 & $0.31342(12)$ & $0.59285(12)$ & $0.23689(10)$ & 0.0189 (3) & 0.932 (3) \\
\hline $\mathrm{H} 8 \mathrm{~A}$ & 0.2708 & 0.5164 & 0.2037 & $0.023^{*}$ & 0.932 (3) \\
\hline $\mathrm{H} 8 \mathrm{~B}$ & 0.2699 & 0.6268 & 0.2777 & $0.023^{*}$ & 0.932 (3) \\
\hline C9 & $0.13783(10)$ & 0.61751 (11) & $-0.00904(8)$ & $0.0174(2)$ & \\
\hline C10 & $0.17627(12)$ & 0.70261 (13) & $0.08255(10)$ & 0.0231 (3) & $0.932(3)$ \\
\hline $\mathrm{H} 10 \mathrm{~A}$ & 0.1659 & 0.7883 & 0.0579 & $0.028^{*}$ & $0.932(3)$ \\
\hline $\mathrm{H} 10 \mathrm{~B}$ & 0.1215 & 0.6895 & 0.1190 & $0.028^{*}$ & $0.932(3)$ \\
\hline C11 & 0.36107 (12) & $0.80236(12)$ & $0.20561(10)$ & 0.0207 (3) & $0.932(3)$ \\
\hline $\mathrm{H} 11 \mathrm{~A}$ & 0.2928 & 0.8603 & 0.1963 & $0.025^{\star}$ & $0.932(3)$ \\
\hline H11B & 0.4102 & 0.7906 & 0.2807 & $0.025^{*}$ & 0.932 (3) \\
\hline C12 & $0.44248(11)$ & $0.85349(10)$ & $0.15727(9)$ & $0.0198(2)$ & \\
\hline $\mathrm{H} 12 \mathrm{~A}$ & 0.4868 & 0.9263 & 0.1965 & $0.024^{*}$ & $0.932(3)$ \\
\hline H12B & 0.3904 & 0.8794 & 0.0855 & $0.024^{*}$ & $0.932(3)$ \\
\hline $\mathrm{H} 12 \mathrm{C}$ & 0.4398 & 0.9206 & 0.1096 & $0.024^{*}$ & $0.068(3)$ \\
\hline H12D & 0.4719 & 0.8886 & 0.2276 & $0.024^{*}$ & 0.068 (3) \\
\hline C8' & $0.3707(13)$ & $0.6658(13)$ & $0.2725(11)$ & $0.011(3)^{*}$ & 0.068 (3) \\
\hline H8'A & 0.4201 & 0.7401 & 0.3029 & $0.013^{*}$ & 0.068 (3) \\
\hline $\mathrm{H} 8{ }^{\prime} \mathrm{B}$ & 0.3052 & 0.6602 & 0.2990 & $0.013^{*}$ & 0.068 (3) \\
\hline C10' & $0.1814(13)$ & $0.6236(13)$ & $0.1139(11)$ & $0.011(3)^{\star}$ & 0.068 (3) \\
\hline $\mathrm{H} 10 \mathrm{C}$ & 0.1225 & 0.6730 & 0.1315 & $0.013^{*}$ & 0.068 (3) \\
\hline H10D & 0.1860 & 0.5405 & 0.1428 & $0.013^{*}$ & 0.068 (3) \\
\hline C11' & $0.3084(15)$ & $0.8044(15)$ & $0.1259(12)$ & $0.017(3)^{*}$ & 0.068 (3) \\
\hline $\mathrm{H} 11 \mathrm{C}$ & 0.2652 & 0.8555 & 0.1585 & $0.020^{*}$ & 0.068 (3) \\
\hline H11D & 0.2621 & 0.8108 & 0.0500 & $0.020^{*}$ & 0.068 (3) \\
\hline O8 & $0.84053(9)$ & $0.61618(12)$ & 0.19501 (8) & $0.0332(2)$ & \\
\hline
\end{tabular}




\begin{tabular}{|l|l|l|l|l|l|}
\hline H81 & $0.8933(16)$ & $0.621(2)$ & $0.2538(8)$ & $0.058(7)^{\star}$ & \\
\hline H82 & $0.8746(17)$ & $0.619(2)$ & $0.1560(12)$ & $0.048(6)^{\star}$ & \\
\hline O9 & $0.77230(11)$ & $0.95110(11)$ & $-0.03463(9)$ & $0.0365(2)$ & \\
\hline H91 & $0.7000(10)$ & $0.9291(19)$ & $-0.0639(19)$ & $0.067(8)^{\star}$ & \\
\hline H92 & $0.8114(15)$ & $0.8871(12)$ & $-0.0234(16)$ & $0.041(5)^{\star}$ & \\
\hline O10 & $0.13509(9)$ & $0.29129(10)$ & $-0.00865(8)$ & $0.0288(2)$ & \\
\hline H101 & $0.2034(10)$ & $0.3214(17)$ & $0.0250(14)$ & $0.042(5)^{\star}$ & \\
\hline H102 & $0.0916(15)$ & $0.3243(18)$ & $0.0166(15)$ & $0.047(6)^{\star}$ & \\
\hline
\end{tabular}

Table S13. Atomic displacement parameters $\left(\AA^{2}\right)$

\begin{tabular}{|l|l|l|l|l|l|l|}
\hline & $U^{11}$ & $U^{2}$ & $U^{\beta 3}$ & $U^{12}$ & $U^{13}$ & $U^{23}$ \\
\hline Zr1 & $0.00987(5)$ & $0.01125(5)$ & $0.00919(5)$ & $0.00046(3)$ & $0.00465(4)$ & $-0.00013(3)$ \\
\hline O1 & $0.0198(4)$ & $0.0154(3)$ & $0.0149(3)$ & $-0.0001(3)$ & $0.0057(3)$ & $0.0023(3)$ \\
\hline O2 & $0.0367(5)$ & $0.0235(4)$ & $0.0218(4)$ & $-0.0045(4)$ & $0.0135(4)$ & $0.0053(3)$ \\
\hline O3 & $0.0162(3)$ & $0.0172(3)$ & $0.0151(3)$ & $-0.0020(3)$ & $0.0058(3)$ & $0.0023(3)$ \\
\hline O4 & $0.0302(4)$ & $0.0279(5)$ & $0.0271(4)$ & $-0.0075(4)$ & $0.0114(4)$ & $0.0090(4)$ \\
\hline O5 & $0.0126(3)$ & $0.0262(4)$ & $0.0155(4)$ & $0.0029(3)$ & $0.0050(3)$ & $-0.0031(3)$ \\
\hline O6 & $0.0128(4)$ & $0.0415(5)$ & $0.0204(4)$ & $0.0033(3)$ & $0.0059(3)$ & $0.0035(4)$ \\
\hline O7 & $0.0102(3)$ & $0.0162(3)$ & $0.0117(3)$ & $-0.0009(3)$ & $0.0042(2)$ & $-0.0029(3)$ \\
\hline N1 & $0.0172(4)$ & $0.0140(4)$ & $0.0136(4)$ & $-0.0012(3)$ & $0.0067(3)$ & $-0.0013(3)$ \\
\hline N2 & $0.0135(4)$ & $0.0170(4)$ & $0.0120(4)$ & $0.0004(3)$ & $0.0057(3)$ & $0.0011(3)$ \\
\hline N3 & $0.0149(4)$ & $0.0194(4)$ & $0.0139(4)$ & $0.0025(3)$ & $0.0064(3)$ & $-0.0012(3)$ \\
\hline C1 & $0.0218(5)$ & $0.0145(5)$ & $0.0175(5)$ & $0.0012(4)$ & $0.0101(4)$ & $0.0007(4)$ \\
\hline C2 & $0.0202(5)$ & $0.0166(5)$ & $0.0180(5)$ & $-0.0041(4)$ & $0.0089(4)$ & $-0.0001(4)$ \\
\hline C3 & $0.0194(5)$ & $0.0200(5)$ & $0.0138(4)$ & $-0.0035(4)$ & $0.0038(4)$ & $-0.0035(4)$ \\
\hline C4 & $0.0140(4)$ & $0.0223(5)$ & $0.0123(4)$ & $-0.0022(4)$ & $0.0029(4)$ & $-0.0004(4)$ \\
\hline C5 & $0.0174(5)$ & $0.0181(5)$ & $0.0173(5)$ & $0.0009(4)$ & $0.0096(4)$ & $0.0022(4)$ \\
\hline C6 & $0.0161(4)$ & $0.0169(5)$ & $0.0163(5)$ & $0.0026(4)$ & $0.0058(4)$ & $0.0043(4)$ \\
\hline
\end{tabular}




\begin{tabular}{|l|l|l|l|l|l|l|}
\hline C7 & $0.0195(5)$ & $0.0264(5)$ & $0.0135(4)$ & $0.0035(4)$ & $0.0094(4)$ & $0.0016(4)$ \\
\hline C8 & $0.0184(5)$ & $0.0242(6)$ & $0.0184(5)$ & $0.0025(4)$ & $0.0118(4)$ & $0.0016(4)$ \\
\hline C9 & $0.0148(4)$ & $0.0225(5)$ & $0.0155(5)$ & $0.0031(4)$ & $0.0069(4)$ & $0.0037(4)$ \\
\hline C10 & $0.0171(5)$ & $0.0313(7)$ & $0.0202(6)$ & $0.0089(5)$ & $0.0068(4)$ & $-0.0022(5)$ \\
\hline C11 & $0.0247(6)$ & $0.0187(6)$ & $0.0206(6)$ & $0.0027(4)$ & $0.0112(5)$ & $-0.0050(4)$ \\
\hline C12 & $0.0259(5)$ & $0.0135(5)$ & $0.0213(5)$ & $0.0023(4)$ & $0.0111(4)$ & $-0.0024(4)$ \\
\hline O8 & $0.0223(4)$ & $0.0549(7)$ & $0.0243(5)$ & $-0.0115(4)$ & $0.0114(4)$ & $-0.0111(5)$ \\
\hline O9 & $0.0382(6)$ & $0.0298(5)$ & $0.0380(6)$ & $0.0007(5)$ & $0.0120(5)$ & $-0.0027(5)$ \\
\hline O10 & $0.0179(4)$ & $0.0323(5)$ & $0.0346(5)$ & $-0.0014(4)$ & $0.0092(4)$ & $-0.0087(4)$ \\
\hline
\end{tabular}

Table S14. Geometric parameters (Å, ํ)

\begin{tabular}{|c|c|c|c|}
\hline $\mathrm{Zr} 1-\mathrm{O} 7$ & $2.0930(7)$ & C4-H4B & 0.9900 \\
\hline Zr1-O5 & $2.1320(8)$ & C5-C6 & $1.5156(15)$ \\
\hline $\mathrm{Zr} 1-\mathrm{O} 1$ & $2.1546(8)$ & $\mathrm{C} 6-\mathrm{H} 6 \mathrm{~A}$ & 0.9900 \\
\hline $\mathrm{Zr} 1-\mathrm{O} 3$ & $2.1714(8)$ & $\mathrm{C} 6-\mathrm{H} 6 \mathrm{~B}$ & 0.9900 \\
\hline $\mathrm{Zr} 1-O 7^{\mathrm{i}}$ & $2.2176(7)$ & C7-C8' & $1.385(14)$ \\
\hline $\mathrm{Zr} 1-\mathrm{N} 2$ & $2.4313(9)$ & $\mathrm{C} 7-\mathrm{C} 8$ & $1.5218(17)$ \\
\hline Zr1-N1 & $2.4355(9)$ & $\mathrm{C} 7-\mathrm{H} 7 \mathrm{~A}$ & 0.9900 \\
\hline $\mathrm{Zr} 1-\mathrm{N} 3$ & $2.4719(9)$ & C7-H7B & 0.9900 \\
\hline $\mathrm{Zr} 1-\mathrm{Zr} 1^{i}$ & 3.5680 (2) & $\mathrm{C} 7-\mathrm{H} 7 \mathrm{C}$ & 0.9900 \\
\hline $\mathrm{O} 1-\mathrm{C} 1$ & $1.2944(14)$ & C7-H7D & 0.9900 \\
\hline $\mathrm{O} 2-\mathrm{C} 1$ & $1.2260(14)$ & $\mathrm{C} 8-\mathrm{H} 8 \mathrm{~A}$ & 0.9900 \\
\hline O3-C5 & $1.3095(13)$ & C8-H8B & 0.9900 \\
\hline $\mathrm{O} 4-\mathrm{C} 5$ & $1.2142(14)$ & $\mathrm{C} 9-\mathrm{C} 10$ & $1.5089(17)$ \\
\hline O5-C9 & $1.2811(14)$ & C9-C10' & $1.604(14)$ \\
\hline O6-C9 & $1.2298(14)$ & C10-H10A & 0.9900 \\
\hline O7-Zr1i & $2.2175(7)$ & C10-H10B & 0.9900 \\
\hline O7-H71 & 0.804 (9) & C11-C12 & $1.4983(18)$ \\
\hline
\end{tabular}




\begin{tabular}{|c|c|c|c|}
\hline $\mathrm{N} 1-\mathrm{C} 2$ & $1.4874(14)$ & $\mathrm{C} 11-\mathrm{H} 11 \mathrm{~A}$ & 0.9900 \\
\hline $\mathrm{N} 1-\mathrm{C} 12$ & $1.4991(14)$ & $\mathrm{C} 11-\mathrm{H} 11 \mathrm{~B}$ & 0.9900 \\
\hline $\mathrm{N} 1-\mathrm{C} 3$ & $1.5053(14)$ & C12-C11' & $1.553(16)$ \\
\hline $\mathrm{N} 2-\mathrm{C} 6$ & $1.4861(14)$ & $\mathrm{C} 12-\mathrm{H} 12 \mathrm{~A}$ & 0.9900 \\
\hline $\mathrm{N} 2-\mathrm{C} 4$ & $1.4906(14)$ & $\mathrm{C} 12-\mathrm{H} 12 \mathrm{~B}$ & 0.9900 \\
\hline $\mathrm{N} 2-\mathrm{C} 7$ & $1.5073(14)$ & $\mathrm{C} 12-\mathrm{H} 12 \mathrm{C}$ & 0.9900 \\
\hline N3-C11' & $1.403(16)$ & $\mathrm{C} 12-\mathrm{H} 12 \mathrm{D}$ & 0.9900 \\
\hline N3-C8 & $1.4897(15)$ & $\mathrm{C} 8^{\prime}-\mathrm{H} 8^{\prime} \mathrm{A}$ & 0.9900 \\
\hline N3-C10 & $1.4903(15)$ & C8'-H8'B & 0.9900 \\
\hline N3-C11 & $1.5008(16)$ & $\mathrm{C} 10^{\prime}-\mathrm{H} 10 \mathrm{C}$ & 0.9900 \\
\hline N3-C10' & 1.506 (14) & C10'-H10D & 0.9900 \\
\hline N3-C8' & $1.520(14)$ & $\mathrm{C} 11^{\prime}-\mathrm{H} 11 \mathrm{C}$ & 0.9900 \\
\hline C1-C2 & $1.5212(15)$ & C11'-H11D & 0.9900 \\
\hline $\mathrm{C} 2-\mathrm{H} 2 \mathrm{~A}$ & 0.9900 & O8-H81 & $0.813(9)$ \\
\hline $\mathrm{C} 2-\mathrm{H} 2 \mathrm{~B}$ & 0.9900 & O8-H82 & $0.807(9)$ \\
\hline C3-C4 & $1.5216(16)$ & $\mathrm{O9-H} 91$ & $0.817(9)$ \\
\hline С3-H3A & 0.9900 & O9-H92 & $0.818(9)$ \\
\hline С3-H3B & 0.9900 & $\mathrm{O} 10-\mathrm{H} 101$ & $0.818(9)$ \\
\hline $\mathrm{C} 4-\mathrm{H} 4 \mathrm{~A}$ & 0.9900 & $\mathrm{O} 10-\mathrm{H} 102$ & $0.820(9)$ \\
\hline O7-Zr1-O5 & 143.51 (3) & N2-C4-H4A & 109.3 \\
\hline O7-Zr1-O1 & 90.64 (3) & $\mathrm{C} 3-\mathrm{C} 4-\mathrm{H} 4 \mathrm{~A}$ & 109.3 \\
\hline $\mathrm{O} 5-\mathrm{Zr} 1-\mathrm{O} 1$ & $81.11(3)$ & $\mathrm{N} 2-\mathrm{C} 4-\mathrm{H} 4 \mathrm{~B}$ & 109.3 \\
\hline O7-Zr1-O3 & 96.77 (3) & $\mathrm{C} 3-\mathrm{C} 4-\mathrm{H} 4 \mathrm{~B}$ & 109.3 \\
\hline O5-Zr1-O3 & 77.21 (3) & $\mathrm{H} 4 \mathrm{~A}-\mathrm{C} 4-\mathrm{H} 4 \mathrm{~B}$ & 108.0 \\
\hline O1-Zr1-O3 & $152.51(3)$ & O4-C5-O3 & $124.58(11)$ \\
\hline O7-Zr1-O7i & $68.30(3)$ & O4-C5-C6 & $123.31(10)$ \\
\hline O5-Zr1-O7i & 75.25 (3) & O3-C5-C6 & $112.10(9)$ \\
\hline O1-Zr1-O7i & 79.52 (3) & N2-C6-C5 & $106.34(9)$ \\
\hline O3-Zr1-O $7^{\mathrm{i}}$ & 78.77 (3) & $\mathrm{N} 2-\mathrm{C} 6-\mathrm{H} 6 \mathrm{~A}$ & 110.5 \\
\hline
\end{tabular}




\begin{tabular}{|c|c|c|c|}
\hline $\mathrm{O} 7-\mathrm{Zr} 1-\mathrm{N} 2$ & $81.26(3)$ & $\mathrm{C} 5-\mathrm{C} 6-\mathrm{H} 6 \mathrm{~A}$ & 110.5 \\
\hline $\mathrm{O} 5-\mathrm{Zr} 1-\mathrm{N} 2$ & $127.15(3)$ & $\mathrm{N} 2-\mathrm{C} 6-\mathrm{H} 6 \mathrm{~B}$ & 110.5 \\
\hline $\mathrm{O} 1-\mathrm{Zr} 1-\mathrm{N} 2$ & $138.50(3)$ & $\mathrm{C} 5-\mathrm{C} 6-\mathrm{H} 6 \mathrm{~B}$ & 110.5 \\
\hline $\mathrm{O} 3-\mathrm{Zr} 1-\mathrm{N} 2$ & 68.95 (3) & $\mathrm{H} 6 \mathrm{~A}-\mathrm{C} 6-\mathrm{H} 6 \mathrm{~B}$ & 108.7 \\
\hline O7i-Zr1-N2 & $132.20(3)$ & $\mathrm{C} 8{ }^{\prime}-\mathrm{C} 7-\mathrm{N} 2$ & $115.0(6)$ \\
\hline $\mathrm{O} 7-\mathrm{Zr} 1-\mathrm{N} 1$ & $83.23(3)$ & N2-C7-C8 & $113.00(9)$ \\
\hline $\mathrm{O} 5-\mathrm{Zr} 1-\mathrm{N} 1$ & $124.98(3)$ & $\mathrm{N} 2-\mathrm{C} 7-\mathrm{H} 7 \mathrm{~A}$ & 109.0 \\
\hline $\mathrm{O} 1-\mathrm{Zr} 1-\mathrm{N} 1$ & $68.52(3)$ & $\mathrm{C} 8-\mathrm{C} 7-\mathrm{H} 7 \mathrm{~A}$ & 109.0 \\
\hline $\mathrm{O} 3-\mathrm{Zr} 1-\mathrm{N} 1$ & 138.55 (3) & $\mathrm{N} 2-\mathrm{C} 7-\mathrm{H} 7 \mathrm{~B}$ & 109.0 \\
\hline O7-Zr1-N1 & $136.71(3)$ & $\mathrm{C} 8-\mathrm{C} 7-\mathrm{H} 7 \mathrm{~B}$ & 109.0 \\
\hline $\mathrm{N} 2-\mathrm{Zr} 1-\mathrm{N} 1$ & 70.12 (3) & $\mathrm{H} 7 \mathrm{~A}-\mathrm{C} 7-\mathrm{H} 7 \mathrm{~B}$ & 107.8 \\
\hline O7-Zr1-N3 & 146.51 (3) & $\mathrm{C} 88^{\prime}-\mathrm{C} 7-\mathrm{H} 7 \mathrm{C}$ & 108.5 \\
\hline O5-Zr1-N3 & 69.98 (3) & $\mathrm{N} 2-\mathrm{C} 7-\mathrm{H} 7 \mathrm{C}$ & 108.5 \\
\hline $\mathrm{O} 1-\mathrm{Zr} 1-\mathrm{N} 3$ & $97.34(3)$ & C8'-C7-H7D & 108.5 \\
\hline $\mathrm{O} 3-\mathrm{Zr} 1-\mathrm{N} 3$ & 90.96 (3) & $\mathrm{N} 2-\mathrm{C} 7-\mathrm{H} 7 \mathrm{D}$ & 108.5 \\
\hline O7i-Zr1-N3 & $145.13(3)$ & $\mathrm{H} 7 \mathrm{C}-\mathrm{C} 7-\mathrm{H} 7 \mathrm{D}$ & 107.5 \\
\hline $\mathrm{N} 2-\mathrm{Zr} 1-\mathrm{N} 3$ & 71.24 (3) & N3-C8-C7 & $110.23(10)$ \\
\hline $\mathrm{N} 1-\mathrm{Zr} 1-\mathrm{N} 3$ & 69.86 (3) & N3-C8-H8A & 109.6 \\
\hline O7-Zr1-Zr1 ${ }^{i}$ & 35.27 (2) & C7-C8-H8A & 109.6 \\
\hline O5-Zr1-Zr1 ${ }^{i}$ & 108.26 (2) & N3-C8-H8B & 109.6 \\
\hline O1-Zr1-Zr1 ${ }^{i}$ & $83.89(2)$ & $\mathrm{C} 7-\mathrm{C} 8-\mathrm{H} 8 \mathrm{~B}$ & 109.6 \\
\hline $\mathrm{O} 3-\mathrm{Zr} 1-\mathrm{Zr} 1^{\mathrm{i}}$ & $87.02(2)$ & $\mathrm{H} 8 \mathrm{~A}-\mathrm{C} 8-\mathrm{H} 8 \mathrm{~B}$ & 108.1 \\
\hline$O 7^{i}-Z r 1-Z r 1^{i}$ & 33.025 (19) & O6-C9-O5 & $124.74(11)$ \\
\hline $\mathrm{N} 2-\mathrm{Zr} 1-\mathrm{Zr} 1^{\mathrm{i}}$ & $109.17(2)$ & $\mathrm{O} 6-\mathrm{C} 9-\mathrm{C} 10$ & $119.57(11)$ \\
\hline $\mathrm{N} 1-\mathrm{Zr} 1-\mathrm{Zr} 1^{\mathrm{i}}$ & $112.54(2)$ & O5-C9-C10 & $115.26(10)$ \\
\hline $\mathrm{N} 3-\mathrm{Zr} 1-\mathrm{Zr} 1^{\mathrm{i}}$ & $177.60(2)$ & $\mathrm{O} 6-\mathrm{C} 9-\mathrm{C} 10^{\prime}$ & 120.0 \\
\hline C1-O1-Zr1 & $123.33(7)$ & O5-C9-C10' & $107.9(5)$ \\
\hline C5-O3-Zr1 & $124.81(7)$ & N3-C10-C9 & $111.61(10)$ \\
\hline $\mathrm{C} 9-\mathrm{O} 5-\mathrm{Zr} 1$ & $129.13(7)$ & N3-C10-H10A & 109.3 \\
\hline
\end{tabular}




\begin{tabular}{|c|c|c|c|}
\hline $\mathrm{Zr} 1-\mathrm{O} 7-\mathrm{Zr} 1^{\mathrm{i}}$ & $111.70(3)$ & C9-C10-H10A & 109.3 \\
\hline $\mathrm{Zr} 1-\mathrm{O} 7-\mathrm{H} 71$ & $132.2(13)$ & N3-C10-H10B & 109.3 \\
\hline 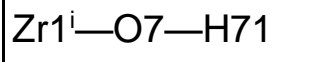 & $115.6(13)$ & C9-C10-H10B & 109.3 \\
\hline $\mathrm{C} 2-\mathrm{N} 1-\mathrm{C} 12$ & $105.93(9)$ & $\mathrm{H} 10 \mathrm{~A}-\mathrm{C} 10-\mathrm{H} 10 \mathrm{~B}$ & 108.0 \\
\hline $\mathrm{C} 2-\mathrm{N} 1-\mathrm{C} 3$ & $111.93(8)$ & $\mathrm{C} 12-\mathrm{C} 11-\mathrm{N} 3$ & $109.54(10)$ \\
\hline $\mathrm{C} 12-\mathrm{N} 1-\mathrm{C} 3$ & $109.33(8)$ & $\mathrm{C} 12-\mathrm{C} 11-\mathrm{H} 11 \mathrm{~A}$ & 109.8 \\
\hline $\mathrm{C} 2-\mathrm{N} 1-\mathrm{Zr} 1$ & $105.01(6)$ & N3-C11-H11A & 109.8 \\
\hline $\mathrm{C} 12-\mathrm{N} 1-\mathrm{Zr} 1$ & $108.29(6)$ & $\mathrm{C} 12-\mathrm{C} 11-\mathrm{H} 11 \mathrm{~B}$ & 109.8 \\
\hline $\mathrm{C} 3-\mathrm{N} 1-\mathrm{Zr} 1$ & $115.83(7)$ & N3-C11-H11B & 109.8 \\
\hline C6-N2-C4 & $109.62(8)$ & $\mathrm{H} 11 \mathrm{~A}-\mathrm{C} 11-\mathrm{H} 11 \mathrm{~B}$ & 108.2 \\
\hline $\mathrm{C} 6-\mathrm{N} 2-\mathrm{C} 7$ & $108.16(9)$ & $\mathrm{C} 11-\mathrm{C} 12-\mathrm{N} 1$ & $110.88(9)$ \\
\hline C4-N2-C7 & $111.36(9)$ & $\mathrm{N} 1-\mathrm{C} 12-\mathrm{C} 11^{\prime}$ & $115.0(6)$ \\
\hline $\mathrm{C} 6-\mathrm{N} 2-\mathrm{Zr} 1$ & $104.63(6)$ & $\mathrm{C} 11-\mathrm{C} 12-\mathrm{H} 12 \mathrm{~A}$ & 109.5 \\
\hline $\mathrm{C} 4-\mathrm{N} 2-\mathrm{Zr} 1$ & $109.91(6)$ & $\mathrm{N} 1-\mathrm{C} 12-\mathrm{H} 12 \mathrm{~A}$ & 109.5 \\
\hline $\mathrm{C} 7-\mathrm{N} 2-\mathrm{Zr} 1$ & $112.89(6)$ & $\mathrm{C} 11-\mathrm{C} 12-\mathrm{H} 12 \mathrm{~B}$ & 109.5 \\
\hline $\mathrm{C} 8-\mathrm{N} 3-\mathrm{C} 10$ & $109.83(10)$ & $\mathrm{N} 1-\mathrm{C} 12-\mathrm{H} 12 \mathrm{~B}$ & 109.5 \\
\hline $\mathrm{C} 8-\mathrm{N} 3-\mathrm{C} 11$ & $109.60(9)$ & $\mathrm{H} 12 \mathrm{~A}-\mathrm{C} 12-\mathrm{H} 12 \mathrm{~B}$ & 108.1 \\
\hline $\mathrm{C} 10-\mathrm{N} 3-\mathrm{C} 11$ & $108.17(10)$ & $\mathrm{N} 1-\mathrm{C} 12-\mathrm{H} 12 \mathrm{C}$ & 108.5 \\
\hline $\mathrm{C} 11^{\prime}-\mathrm{N} 3-\mathrm{C} 10^{\prime}$ & $114.4(9)$ & $\mathrm{C} 11^{\prime}-\mathrm{C} 12-\mathrm{H} 12 \mathrm{C}$ & 108.5 \\
\hline $\mathrm{C} 11^{\prime}-\mathrm{N} 3-\mathrm{C} 8^{\prime}$ & $112.5(8)$ & $\mathrm{N} 1-\mathrm{C} 12-\mathrm{H} 12 \mathrm{D}$ & 108.5 \\
\hline C10'-N3-C8' & $110.0(8)$ & C11'-C12-H12D & 108.5 \\
\hline $\mathrm{C} 11^{\prime}-\mathrm{N} 3-\mathrm{Zr} 1$ & $101.9(7)$ & $\mathrm{H} 12 \mathrm{C}-\mathrm{C} 12-\mathrm{H} 12 \mathrm{D}$ & 107.5 \\
\hline $\mathrm{C} 8-\mathrm{N} 3-\mathrm{Zr} 1$ & $103.72(7)$ & $\mathrm{C} 7-\mathrm{C} 8{ }^{\prime}-\mathrm{N} 3$ & $116.4(10)$ \\
\hline $\mathrm{C} 10-\mathrm{N} 3-\mathrm{Zr} 1$ & $109.94(7)$ & $\mathrm{C} 7-\mathrm{C} 8^{\prime}-\mathrm{H} 8^{\prime} \mathrm{A}$ & 108.2 \\
\hline $\mathrm{C} 11-\mathrm{N} 3-\mathrm{Zr} 1$ & $115.46(7)$ & $\mathrm{N} 3-\mathrm{C} 8^{\prime}-\mathrm{H} 8^{\prime} \mathrm{A}$ & 108.2 \\
\hline $\mathrm{C} 10^{\prime}-\mathrm{N} 3-\mathrm{Zr} 1$ & $105.3(5)$ & $\mathrm{C} 7-\mathrm{C} 8^{\prime}-\mathrm{H} 8^{\prime} \mathrm{B}$ & 108.2 \\
\hline $\mathrm{C} 8{ }^{\prime}-\mathrm{N} 3-\mathrm{Zr} 1$ & $112.4(5)$ & $\mathrm{N} 3-\mathrm{C} 8^{\prime}-\mathrm{H} 8^{\prime} \mathrm{B}$ & 108.2 \\
\hline $\mathrm{O} 2-\mathrm{C} 1-\mathrm{O} 1$ & $124.60(11)$ & H8'A-C8'-H8'B & 107.3 \\
\hline $\mathrm{O} 2-\mathrm{C} 1-\mathrm{C} 2$ & $120.65(10)$ & N3-C10'-C9 & $105.8(8)$ \\
\hline
\end{tabular}




\begin{tabular}{|c|c|c|c|}
\hline $\mathrm{O} 1-\mathrm{C} 1-\mathrm{C} 2$ & $114.76(9)$ & N3-C10'-H10C & 110.6 \\
\hline $\mathrm{N} 1-\mathrm{C} 2-\mathrm{C} 1$ & $108.15(9)$ & $\mathrm{C} 9-\mathrm{C} 10^{\prime}-\mathrm{H} 10 \mathrm{C}$ & 110.6 \\
\hline $\mathrm{N} 1-\mathrm{C} 2-\mathrm{H} 2 \mathrm{~A}$ & 110.1 & N3-C10'-H10D & 110.6 \\
\hline $\mathrm{C} 1-\mathrm{C} 2-\mathrm{H} 2 \mathrm{~A}$ & 110.1 & C9-C10'-H10D & 110.6 \\
\hline $\mathrm{N} 1-\mathrm{C} 2-\mathrm{H} 2 \mathrm{~B}$ & 110.1 & $\mathrm{H} 10 \mathrm{C}-\mathrm{C} 10^{\prime}-\mathrm{H} 10 \mathrm{D}$ & 108.7 \\
\hline $\mathrm{C} 1-\mathrm{C} 2-\mathrm{H} 2 \mathrm{~B}$ & 110.1 & N3-C11'-C12 & $111.9(10)$ \\
\hline $\mathrm{H} 2 \mathrm{~A}-\mathrm{C} 2-\mathrm{H} 2 \mathrm{~B}$ & 108.4 & N3-C11'-H11C & 109.2 \\
\hline $\mathrm{N} 1-\mathrm{C} 3-\mathrm{C} 4$ & $112.71(9)$ & $\mathrm{C} 12-\mathrm{C} 11^{\prime}-\mathrm{H} 11 \mathrm{C}$ & 109.2 \\
\hline $\mathrm{N} 1-\mathrm{C} 3-\mathrm{H} 3 \mathrm{~A}$ & 109.0 & N3-C11'-H11D & 109.2 \\
\hline $\mathrm{C} 4-\mathrm{C} 3-\mathrm{H} 3 \mathrm{~A}$ & 109.0 & C12-C11'-H11D & 109.2 \\
\hline $\mathrm{N} 1-\mathrm{C} 3-\mathrm{H} 3 \mathrm{~B}$ & 109.0 & $\mathrm{H} 11 \mathrm{C}-\mathrm{C} 11^{\prime}-\mathrm{H} 11 \mathrm{D}$ & 107.9 \\
\hline C4-C3-H3B & 109.0 & H81-O8-H82 & $108.3(17)$ \\
\hline $\mathrm{H} 3 \mathrm{~A}-\mathrm{C} 3-\mathrm{H} 3 \mathrm{~B}$ & 107.8 & H91-O9-H92 & $103.6(17)$ \\
\hline $\mathrm{N} 2-\mathrm{C} 4-\mathrm{C} 3$ & $111.60(9)$ & $\mathrm{H} 101-\mathrm{O} 10-\mathrm{H} 102$ & $102.1(16)$ \\
\hline $\mathrm{Zr} 1-\mathrm{O} 1-\mathrm{C} 1-\mathrm{O} 2$ & $166.26(9)$ & $\mathrm{Zr} 1-\mathrm{O} 5-\mathrm{C9}-\mathrm{O} 6$ & $-177.79(9)$ \\
\hline $\mathrm{Zr} 1-\mathrm{O} 1-\mathrm{C} 1-\mathrm{C} 2$ & $-13.66(13)$ & $\mathrm{Zr} 1-\mathrm{O} 5-\mathrm{C} 9-\mathrm{C} 10$ & $9.74(15)$ \\
\hline $\mathrm{C} 12-\mathrm{N} 1-\mathrm{C} 2-\mathrm{C} 1$ & $-71.30(10)$ & $\mathrm{Zr} 1-\mathrm{O} 5-\mathrm{C} 9-\mathrm{C} 10^{\prime}$ & $-28.1(6)$ \\
\hline $\mathrm{C} 3-\mathrm{N} 1-\mathrm{C} 2-\mathrm{C} 1$ & $169.63(9)$ & $\mathrm{C} 8-\mathrm{N} 3-\mathrm{C} 10-\mathrm{C} 9$ & $-92.35(12)$ \\
\hline $\mathrm{Zr} 1-\mathrm{N} 1-\mathrm{C} 2-\mathrm{C} 1$ & $43.18(9)$ & $\mathrm{C} 11-\mathrm{N} 3-\mathrm{C} 10-\mathrm{C} 9$ & $148.06(11)$ \\
\hline $\mathrm{O} 2-\mathrm{C} 1-\mathrm{C} 2-\mathrm{N} 1$ & $155.90(11)$ & Zr1-N3-C10-C9 & 21.16 (13) \\
\hline $\mathrm{O} 1-\mathrm{C} 1-\mathrm{C} 2-\mathrm{N} 1$ & $-24.18(13)$ & $\mathrm{O} 6-\mathrm{C} 9-\mathrm{C} 10-\mathrm{N} 3$ & $166.12(11)$ \\
\hline $\mathrm{C} 2-\mathrm{N} 1-\mathrm{C} 3-\mathrm{C} 4$ & $-114.42(10)$ & $\mathrm{O} 5-\mathrm{C} 9-\mathrm{C} 10-\mathrm{N} 3$ & $-20.99(16)$ \\
\hline $\mathrm{C} 12-\mathrm{N} 1-\mathrm{C} 3-\mathrm{C} 4$ & $128.54(10)$ & $\mathrm{C} 8-\mathrm{N} 3-\mathrm{C} 11-\mathrm{C} 12$ & $137.31(10)$ \\
\hline $\mathrm{Zr} 1-\mathrm{N} 1-\mathrm{C} 3-\mathrm{C} 4$ & $5.92(12)$ & $\mathrm{C} 10-\mathrm{N} 3-\mathrm{C} 11-\mathrm{C} 12$ & $-102.96(11)$ \\
\hline $\mathrm{C} 6-\mathrm{N} 2-\mathrm{C} 4-\mathrm{C} 3$ & $164.89(9)$ & $\mathrm{Zr} 1-\mathrm{N} 3-\mathrm{C} 11-\mathrm{C} 12$ & $20.67(12)$ \\
\hline C7-N2-C4-C3 & $-75.45(11)$ & N3-C11-C12-N1 & $-51.06(13)$ \\
\hline $\mathrm{Zr} 1-\mathrm{N} 2-\mathrm{C} 4-\mathrm{C} 3$ & $50.41(10)$ & $\mathrm{C} 2-\mathrm{N} 1-\mathrm{C} 12-\mathrm{C} 11$ & $168.66(9)$ \\
\hline $\mathrm{N} 1-\mathrm{C} 3-\mathrm{C} 4-\mathrm{N} 2$ & $-37.23(13)$ & C3-N1-C12-C11 & $-70.57(11)$ \\
\hline $\mathrm{Zr} 1-\mathrm{O} 3-\mathrm{C} 5-\mathrm{O} 4$ & $169.35(9)$ & $\mathrm{Zr} 1-\mathrm{N} 1-\mathrm{C} 12-\mathrm{C} 11$ & $56.45(10)$ \\
\hline
\end{tabular}




\begin{tabular}{|c|c|c|c|}
\hline $\mathrm{Zr} 1-\mathrm{O} 3-\mathrm{C} 5-\mathrm{C} 6$ & $-9.66(13)$ & $\mathrm{C} 2-\mathrm{N} 1-\mathrm{C} 12-\mathrm{C} 11^{\prime}$ & $125.3(7)$ \\
\hline $\mathrm{C} 4-\mathrm{N} 2-\mathrm{C} 6-\mathrm{C} 5$ & $-168.28(9)$ & C3-N1-C12-C11' & $-113.9(7)$ \\
\hline $\mathrm{C} 7-\mathrm{N} 2-\mathrm{C} 6-\mathrm{C} 5$ & $70.12(10)$ & $\mathrm{Zr} 1-\mathrm{N} 1-\mathrm{C} 12-\mathrm{C} 11^{\prime}$ & $13.1(7)$ \\
\hline $\mathrm{Zr} 1-\mathrm{N} 2-\mathrm{C} 6-\mathrm{C} 5$ & $-50.45(9)$ & $\mathrm{N} 2-\mathrm{C} 7-\mathrm{C} 8 \mathrm{-}-\mathrm{N} 3$ & $40.0(11)$ \\
\hline $\mathrm{O} 4-\mathrm{C} 5-\mathrm{C} 6-\mathrm{N} 2$ & $-136.71(12)$ & $\mathrm{C} 11^{\prime}-\mathrm{N} 3-\mathrm{C} 8 \mathrm{\prime}-\mathrm{C} 7$ & $-137.8(11)$ \\
\hline $\mathrm{O} 3-\mathrm{C} 5-\mathrm{C} 6-\mathrm{N} 2$ & $42.32(12)$ & $\mathrm{C} 10^{\prime}-\mathrm{N} 3-\mathrm{C} 8 \mathrm{\prime}-\mathrm{C} 7$ & $93.4(11)$ \\
\hline $\mathrm{C} 6-\mathrm{N} 2-\mathrm{C} 7-\mathrm{C} 8$ & $-151.3(6)$ & $\mathrm{Zr} 1-\mathrm{N} 3-\mathrm{C} 8 \mathrm{\prime}-\mathrm{C} 7$ & $-23.4(11)$ \\
\hline $\mathrm{C} 4-\mathrm{N} 2-\mathrm{C} 7-\mathrm{C} 8$ & $88.2(6)$ & $\mathrm{C} 11^{\prime}-\mathrm{N} 3-\mathrm{C} 10^{\prime}-\mathrm{C} 9$ & $65.5(11)$ \\
\hline $\mathrm{Zr} 1-\mathrm{N} 2-\mathrm{C} 7-\mathrm{C} 8$ & $-36.0(6)$ & $\mathrm{C} 8{ }^{\prime}-\mathrm{N} 3-\mathrm{C} 10^{\prime}-\mathrm{C} 9$ & $-166.8(8)$ \\
\hline $\mathrm{C} 6-\mathrm{N} 2-\mathrm{C} 7-\mathrm{C} 8$ & $-105.91(11)$ & $\mathrm{Zr} 1-\mathrm{N} 3-\mathrm{C} 10^{\prime}-\mathrm{C} 9$ & $-45.5(8)$ \\
\hline $\mathrm{C} 4-\mathrm{N} 2-\mathrm{C} 7-\mathrm{C} 8$ & $133.57(10)$ & $\mathrm{O} 6-\mathrm{C} 9-\mathrm{C} 10^{\prime}-\mathrm{N} 3$ & $-159.9(5)$ \\
\hline $\mathrm{Zr} 1-\mathrm{N} 2-\mathrm{C} 7-\mathrm{C} 8$ & $9.37(12)$ & $\mathrm{O} 5-\mathrm{C} 9-\mathrm{C} 10^{\prime}-\mathrm{N} 3$ & $48.8(8)$ \\
\hline $\mathrm{C} 10-\mathrm{N} 3-\mathrm{C} 8-\mathrm{C} 7$ & $176.36(10)$ & C10'-N3-C11'-C12 & $-171.2(9)$ \\
\hline $\mathrm{C} 11-\mathrm{N} 3-\mathrm{C} 8-\mathrm{C} 7$ & $-64.94(12)$ & $\mathrm{C} 8{ }^{\prime}-\mathrm{N} 3-\mathrm{C} 11^{\prime}-\mathrm{C} 12$ & $62.4(12)$ \\
\hline $\mathrm{Zr} 1-\mathrm{N} 3-\mathrm{C} 8-\mathrm{C} 7$ & $58.89(10)$ & $\mathrm{Zr} 1-\mathrm{N} 3-\mathrm{C} 11^{\prime}-\mathrm{C} 12$ & $-58.2(10)$ \\
\hline $\mathrm{N} 2-\mathrm{C} 7-\mathrm{C} 8-\mathrm{N} 3$ & $-47.53(13)$ & $\mathrm{N} 1-\mathrm{C} 12-\mathrm{C} 11^{\prime}-\mathrm{N} 3$ & $32.5(12)$ \\
\hline
\end{tabular}

Symmetry code: (i) $-x+1,-y+1,-z$.

Table S15. Hydrogen-bond geometry $(\AA \stackrel{\circ}{\circ})$

\begin{tabular}{|l|l|l|l|l|}
\hline$D-\mathrm{H} \cdots A$ & $D-\mathrm{H}$ & $\mathrm{H} \cdots A$ & $D \cdots A$ & $D-\mathrm{H} \cdots A$ \\
\hline $\mathrm{O} 7-\mathrm{H} 71 \cdots \mathrm{O} 8$ & $0.80(1)$ & $2.06(1)$ & $2.8594(12)$ & $172(2)$ \\
\hline $\mathrm{O} 8-\mathrm{H} 81 \cdots \mathrm{O} 2^{\mathrm{ii}}$ & $0.81(1)$ & $2.08(1)$ & $2.8792(14)$ & $169(2)$ \\
\hline $\mathrm{O} 8-\mathrm{H} 82 \cdots \mathrm{O} 10^{\mathrm{i}}$ & $0.81(1)$ & $2.27(1)$ & $2.9522(16)$ & $143(2)$ \\
\hline $\mathrm{O}-\mathrm{H} 91 \cdots \mathrm{O} 2$ & $0.82(1)$ & $2.30(1)$ & $3.0776(16)$ & $160(3)$ \\
\hline $\mathrm{O}-\mathrm{H} 92 \cdots \mathrm{O} 10^{\mathrm{i}}$ & $0.82(1)$ & $2.04(1)$ & $2.8378(16)$ & $164(2)$ \\
\hline $\mathrm{O} 10-\mathrm{H} 101 \cdots \mathrm{O} 3$ & $0.82(1)$ & $2.02(1)$ & $2.8404(12)$ & $178(2)$ \\
\hline $\mathrm{O} 10-\mathrm{H} 102 \cdots \mathrm{O}^{\mathrm{iii}}$ & $0.82(1)$ & $1.96(1)$ & $2.7766(14)$ & $174(2)$ \\
\hline
\end{tabular}

Symmetry codes: (i) $-x+1,-y+1,-z$; (ii) $x+1 / 2,-y+3 / 2, z+1 / 2$; (iii) $-x,-y+1,-z$. 
Least-squares planes ( $x, y, z$ in crystal coordinates) and deviations from them (* indicates atom used to define plane).

$-1.8012(0.0053) x+5.1785(0.0044) y+12.1093(0.0034) z=4.8668(0.0034)$

* $0.0000(0.0000) \mathrm{N} 1$

* $0.0000(0.0000) \mathrm{N} 2$

* $0.0000(0.0000) \mathrm{N} 3$

$-1.8253(0.0005) \mathrm{Zr} 1$

$-2.3383(0.0013) 01$

$-2.2028(0.0014) 03$

$-2.6372(0.0013) 05$

$-2.3353(0.0013) 07$

Rms deviation of fitted atoms $=0.0000$

$-3.9828(0.0061) x+5.8162(0.0026) y+12.0108(0.0022) z=2.1012(0.0025)$

Angle to previous plane (with approximate esd) $=12.290(0.049)$

* $0.0000(0.0000) 01$

* $0.0000(0.0000) 03$

* $0.0000(0.0000) 05$

$0.3820(0.0007) \mathrm{Zr} 1$

$-0.5400(0.0018) 07$

$2.0675(0.0016) \mathrm{N} 1$

$1.9331(0.0017) \mathrm{N} 2$

$2.5134(0.0011) \mathrm{N} 3$

Rms deviation of fitted atoms $=0.0000$ 
$-2.4457(0.0031) x+5.6205(0.0026) y+11.9740(0.0022) z=2.4723(0.0020)$

Angle to previous plane (with approximate esd) $=8.192(0.039)$

* $0.1179(0.0004) 01$

* $0.1161(0.0004) \mathrm{O}$

* $-0.1327(0.0005) 05$

* $\quad-0.1013(0.0003) 07$

$0.5441(0.0004) \mathrm{Zr} 1$

$2.3644(0.0011) \mathrm{N} 1$

$2.2583(0.0011) \mathrm{N} 2$

$2.4764(0.0011)$ N3

Rms deviation of fitted atoms $=0.1175$ 
<smiles>O=C(O)CN1CCN(CC(=O)O)Cc2cccc(n2)CN(CC(=O)O)CC1</smiles>

PCTA<smiles>O=C(O)CN1CCN(CC(=O)O)CCN(CC(=O)O)CC1</smiles>

NOTA<smiles>O=C(O)CN1CCCN(CC(=O)O)CCN(CC(=O)O)CCC1</smiles>

TRITA<smiles>C[R16](=O)O[Na]</smiles>
$99{ }^{\circ} \mathrm{C}, 2 \mathrm{~h}$

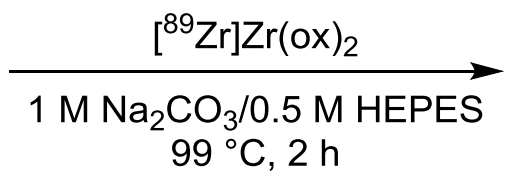

$\underset{1 \mathrm{M} \mathrm{Na}_{2} \mathrm{CO}_{3} / 0.5 \mathrm{M} \text { HEPES }}{\stackrel{\left[{ }^{89} \mathrm{Zr}\right] \mathrm{Zr}(\mathrm{ox})_{2}}{\longrightarrow}}$ $99^{\circ} \mathrm{C}, 2 \mathrm{~h}$

$\left[{ }^{89} \mathrm{Zr}\right] \mathrm{Zr}(\mathrm{ox})_{2}$ $\longrightarrow$

$1 \mathrm{M} \mathrm{Na}_{2} \mathrm{CO}_{3} / 0.5 \mathrm{M}$ HEPES $99{ }^{\circ} \mathrm{C}, 2 \mathrm{~h}$

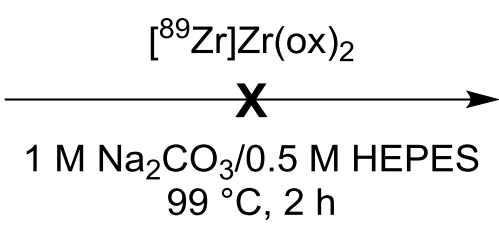

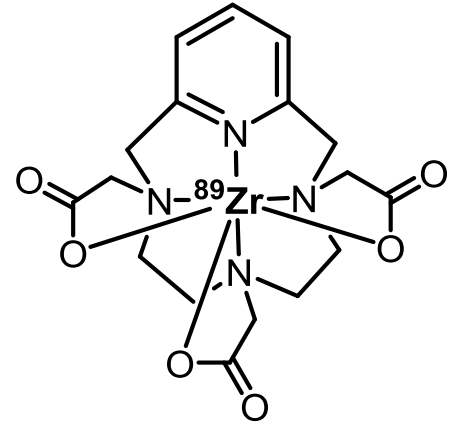

$\left[{ }^{89} \mathrm{Zr}\right] \mathrm{Zr}-\mathrm{PCTA}$

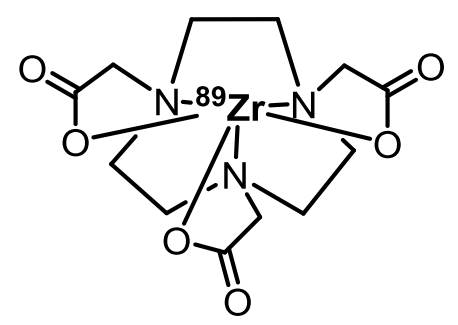

$\left[{ }^{89} \mathrm{Zr}\right] \mathrm{Zr}-\mathrm{NOTA}$

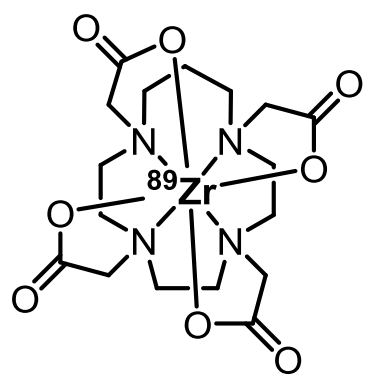

[ $\left.{ }^{89} \mathrm{Zr}\right] \mathrm{Zr}-\mathrm{TRITA}$<smiles>O=C(O)CN1CCCN(CC(=O)O)CCCN(CC(=O)O)CCC1</smiles>

TETA

Scheme S5. Radiochemical Synthesis of $\left[{ }^{89} \mathrm{Zr}\right] \mathrm{Zr}$-PCTA, $\left.{ }^{89} \mathrm{Zr}\right] \mathrm{Zr}-\mathrm{NOTA},\left[{ }^{89} \mathrm{Zr}\right] \mathrm{Zr}$-TRITA and $\left[{ }^{89} \mathrm{Zr}\right] \mathrm{Zr}$ TETA using $\left.{ }^{89} \mathrm{Zr}\right] \mathrm{Zr}(\mathrm{ox})_{2}$ 
Table S16. Summary of optimized radiochemistry conditions to prepare ${ }^{89} \mathrm{Zr}$-complexes with $\left[{ }^{89} \mathrm{Zr}\right] \mathrm{Zr}(\mathrm{ox})_{2}$

\begin{tabular}{ccccc}
\hline \multirow{2}{*}{ Radiochemistry conditions } & \multicolumn{3}{c}{ Ligands $(\mathbf{n}=10)$} \\
\cline { 2 - 5 } & PCTA & NOTA & TRITA & TETA \\
\hline Quantity $(\mu \mathrm{g})$ & $10-50$ & $10-50$ & $10-50$ & $10-50$ \\
{$\left[{ }^{89} \mathrm{Zr}\right] \mathrm{Zr}(\mathrm{ox})_{2}$ added $(\mathrm{MBq})$} & $16.5-20.5$ & $16.5-20.5$ & $16.5-20.5$ & $16.5-20.5$ \\
Reaction Buffer & $0.5 \mathrm{M} \mathrm{HEPES}$ & $0.5 \mathrm{M} \mathrm{HEPES}$ & 0.5 M HEPES & 0.5 M HEPES \\
Final Reaction pH & $6.9-7.3$ & $6.9-7.3$ & $6.9-7.3$ & $6.9-7.3$ \\
Reaction Temperature $\left({ }^{\circ} \mathrm{C}\right)$ & 99 & 99 & 99 & 99 \\
Reaction time $(\mathrm{min})$ & 120 & 120 & 120 & 120 \\
Radiochemical yield $(\%)$ & $35 \pm 9.3$ & $15 \pm 5.7$ & $3 \pm 1.3$ & 0 \\
\hline
\end{tabular}




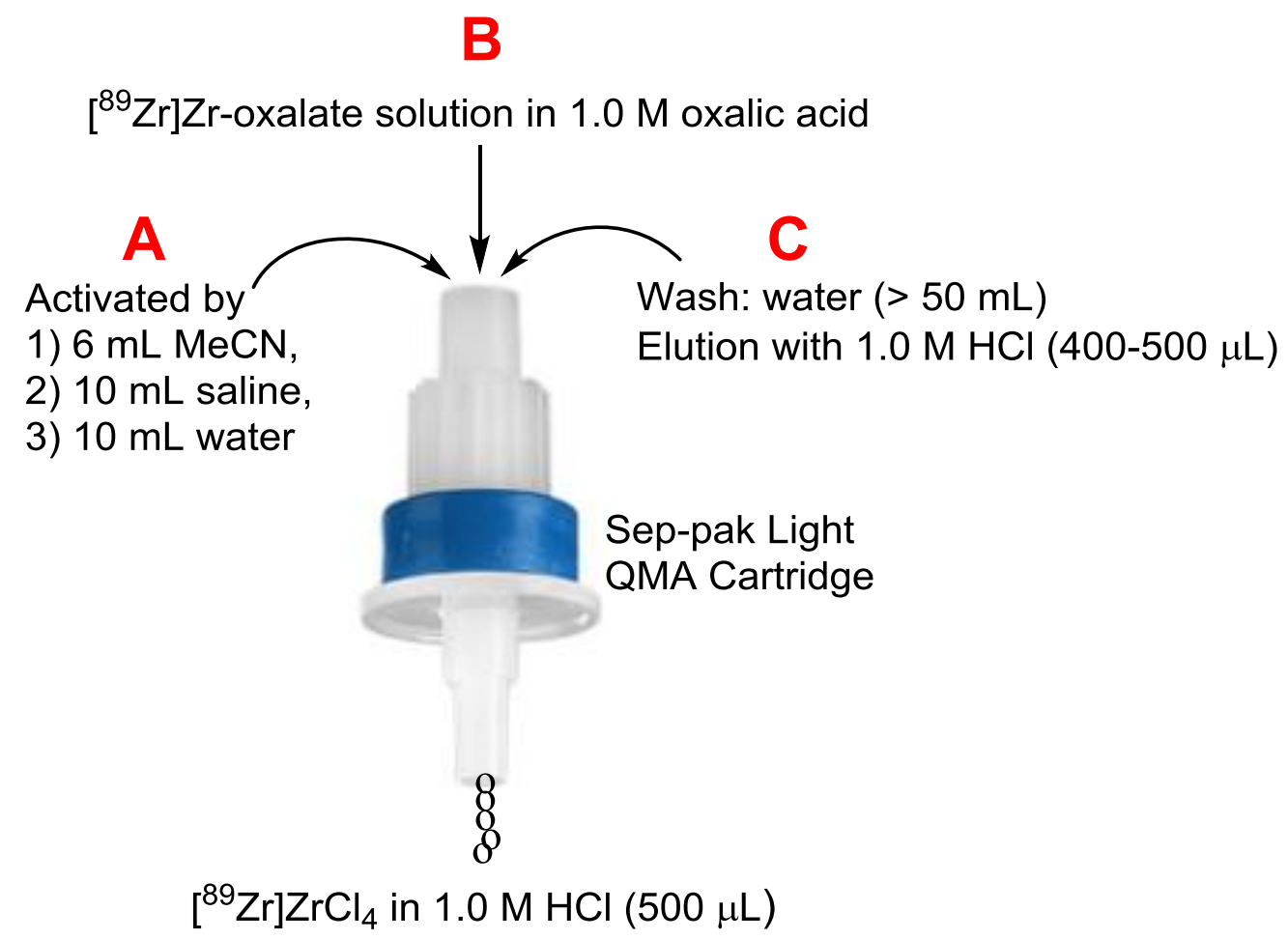

Figure S42. Schematic diagram for the production of $\left[{ }^{89} \mathrm{Zr}\right] \mathrm{ZrCl}_{4}$ from $\left[{ }^{89} \mathrm{Zr}\right] \mathrm{Zr}(\mathrm{ox})_{2}$ 
<smiles>O=C(O)CN1CCN(CC(=O)O)Cc2cccc(n2)CN(CC(=O)O)CC1</smiles>

PCTA<smiles>O=C(O)CN1CCN(CC(=O)O)CCN(CC(=O)O)CC1</smiles>

NOTA<smiles>O=C(O)CN1CCCN(CC(=O)O)N(CC(=O)O)CCCN(CC(=O)O)CC1</smiles>

TRITA<smiles>O=C(O)CN1CCCN(CC(=O)O)CCCN(CC(=O)O)N(CC(=O)O)CCC1</smiles>

TETA

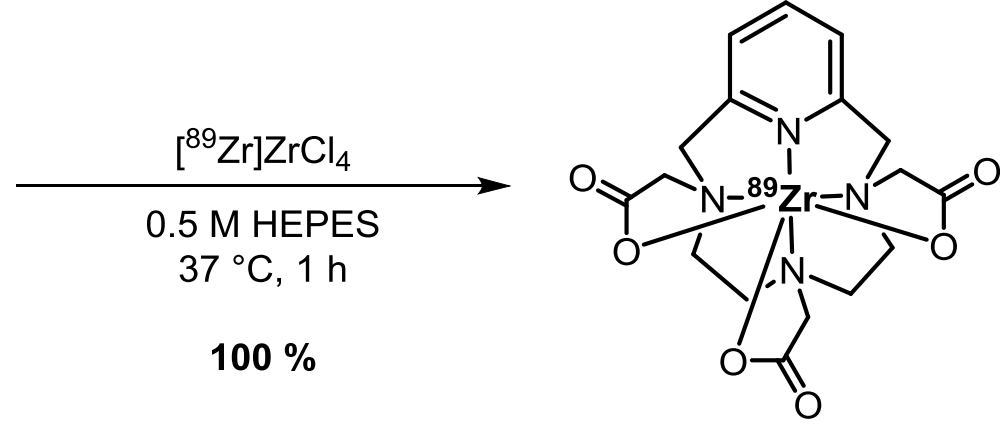

$\left[{ }^{89} \mathrm{Zr}\right] \mathrm{Zr}-\mathrm{PCTA}$

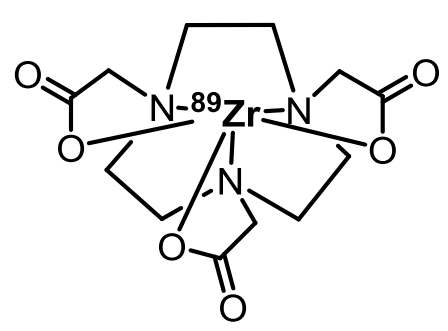

[ $\left.{ }^{89} \mathrm{Zr}\right] \mathrm{Zr}$-NOTA

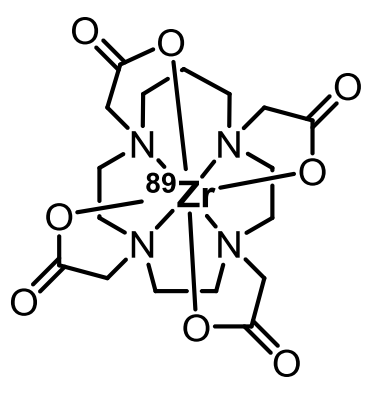

[ $\left.{ }^{89} \mathrm{Zr}\right] \mathrm{Zr}$-TRITA

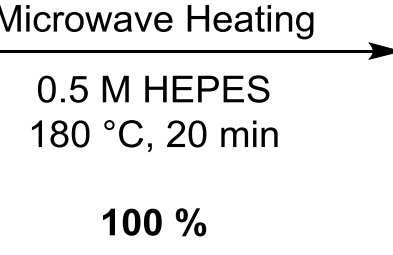

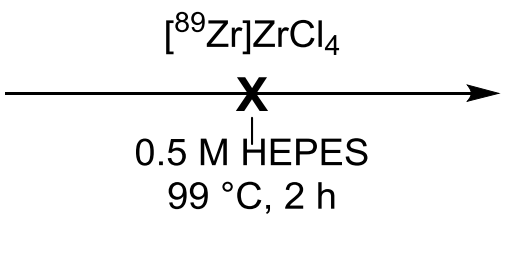

Scheme S6. Radiochemical Synthesis of [ $\left.{ }^{89} \mathrm{Zr}\right] \mathrm{Zr}-\mathrm{PCTA},\left[{ }^{89} \mathrm{Zr}\right] \mathrm{Zr}-\mathrm{NOTA}$, [ $\left.{ }^{89} \mathrm{Zr}\right] \mathrm{Zr}-\mathrm{TRITA}$ and $\left[{ }^{89} \mathrm{Zr}\right] \mathrm{Zr}$ TETA using $\left[{ }^{89} \mathrm{Zr}\right] \mathrm{ZrCl}_{4}$ 
Radiochemical Synthesis of $\left[{ }^{89} \mathrm{Zr}\right] \mathrm{Zr}$-PCTA with $\left[{ }^{89} \mathrm{Zr}\right] \mathrm{ZrCl}_{4}$ :

Table S17. Summary of optimized radiochemistry conditions and $\left[{ }^{89} \mathrm{Zr}^{2} \mathrm{ZrCl}_{4}\right.$ activity used to prepare $\left[{ }^{89} \mathrm{Zr}\right] \mathrm{Zr}-\mathrm{PCTA}{ }^{\mathrm{a}}$ complex $(\mathrm{n}=4$ for each buffer)

\begin{tabular}{|c|c|c|c|c|c|}
\hline $\begin{array}{l}\text { Quantity of } \\
\text { Ligand }\end{array}$ & $\begin{array}{c}{\left[{ }^{89} \mathrm{Zr}\right] \mathrm{ZrCl}_{4}} \\
\text { added }(\mathrm{MBq})\end{array}$ & $\begin{array}{l}\text { Reaction Buffer } \\
\text { (pH } 6.8-7.2)\end{array}$ & $\begin{array}{c}\text { Reaction } \\
\text { Temperature } \\
\left({ }^{\circ} \mathrm{C}\right)\end{array}$ & $\begin{array}{l}\text { Reaction } \\
\text { Time (min) }\end{array}$ & $\begin{array}{l}\text { Radiochemical Yield } \\
\text { by Radio-ITLC (\%) }\end{array}$ \\
\hline \multirow{8}{*}{$\begin{array}{c}\text { PCTA } \\
(7-10 \mu \mathrm{g})\end{array}$} & 18.5 & $1 \mathrm{M} \mathrm{NH}_{4} \mathrm{OAc}$ & 37 & 60 & $77.7 \pm 0.9$ \\
\hline & 19.1 & $1 \mathrm{M} \mathrm{NaOAc}$ & 37 & 60 & $59.1 \pm 1.1$ \\
\hline & 18.3 & $1 \mathrm{M}$ TRIS & 37 & 60 & $27.2 \pm 1.3$ \\
\hline & 18.4 & $1 \mathrm{M}$ TMAA & 37 & 60 & $15.9 \pm 0.8$ \\
\hline & 19.2 & $0.5 \mathrm{M}$ MES & 37 & 60 & $93.1 \pm 0.7$ \\
\hline & 18.8 & $0.5 \mathrm{M}$ HEPES & 37 & 60 & $99.9 \pm 0.1$ \\
\hline & 18.5 & $0.5 \mathrm{M}$ HEPES & 24 & 60 & $70.8 \pm 0.5$ \\
\hline & 18.4 & 0.5 M HEPES & 24 & 120 & $92.3 \pm 0.6$ \\
\hline
\end{tabular}

aPCTA ligand $(7-10 \mu \mathrm{g})$ was labeled with $\left[{ }^{89} \mathrm{Zr}\right] \mathrm{ZrCl}_{4}(18.3-19.2 \mathrm{MBq})$ using buffers $(200 \mu \mathrm{L}, \mathrm{pH}$ 6.8 - 7.2) at $24^{\circ} \mathrm{C}$ or $37^{\circ} \mathrm{C}$ for $60-120 \mathrm{~min}$. 
(a)

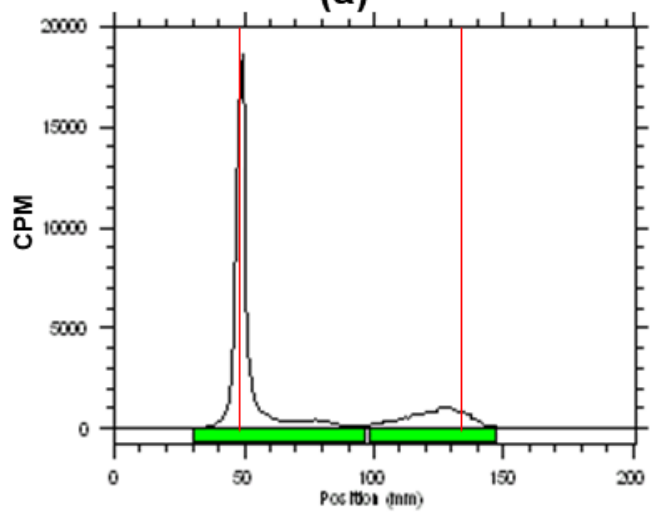

(d)

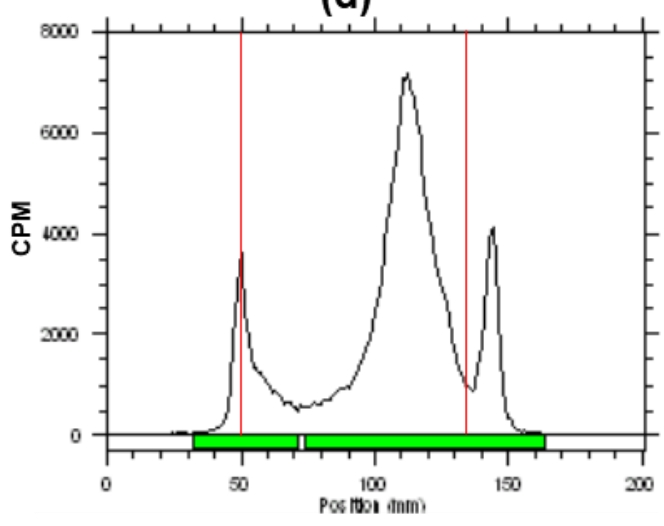

(g)

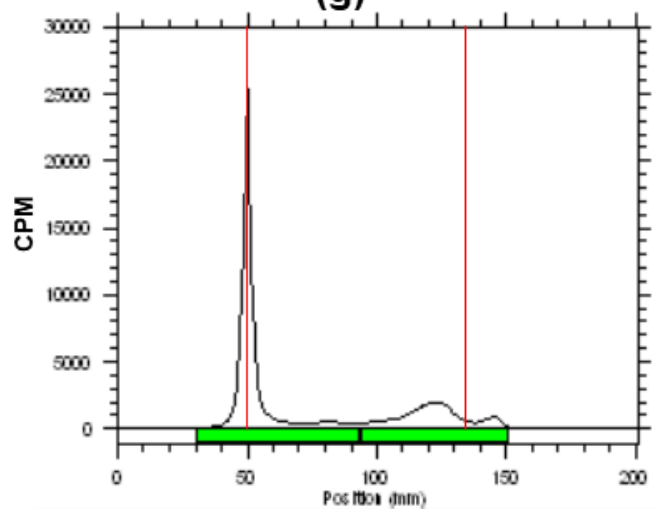

(b)

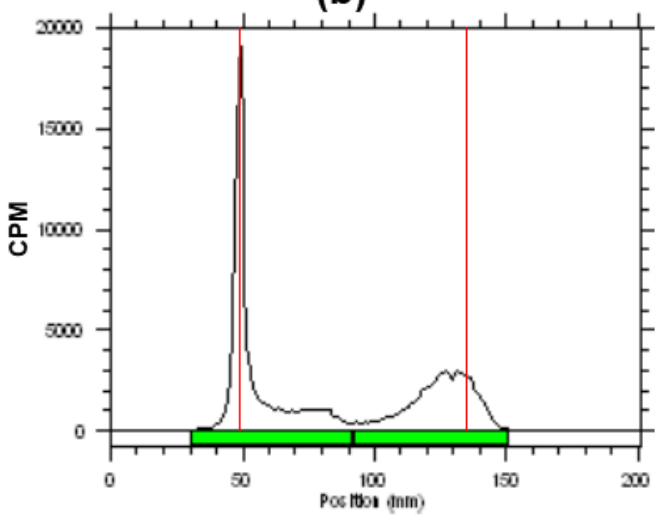

(e)

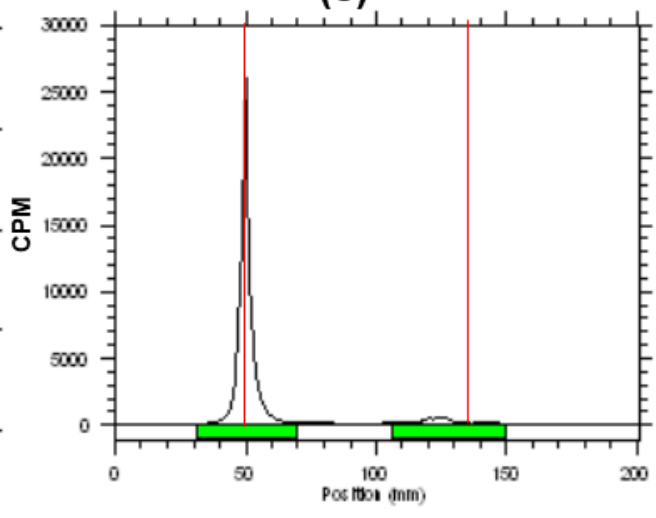

(h)

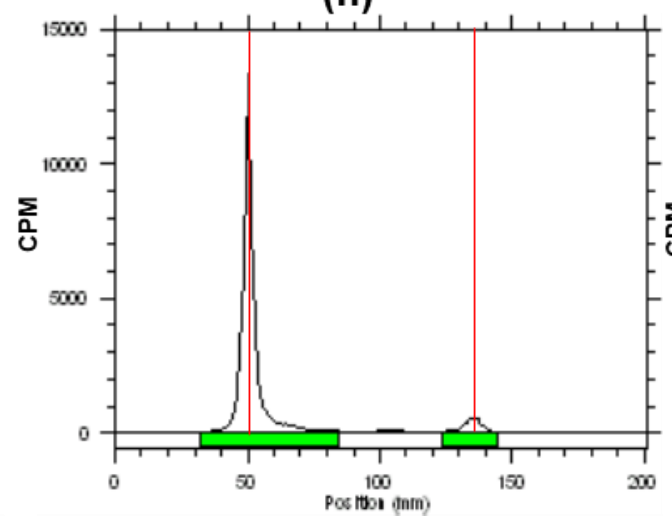

(c)

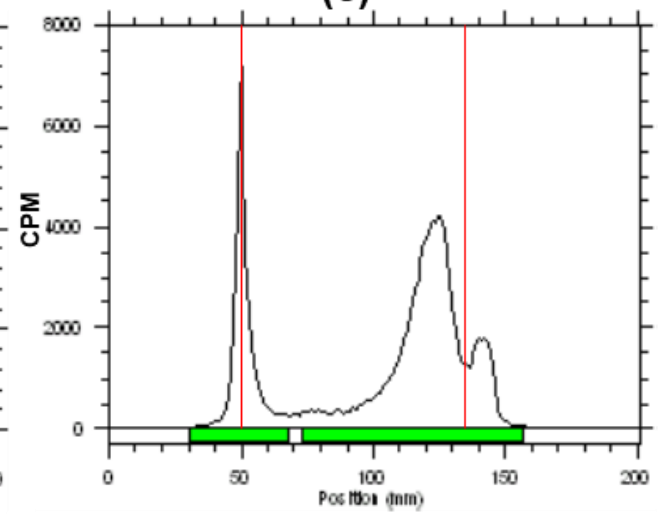

(f)

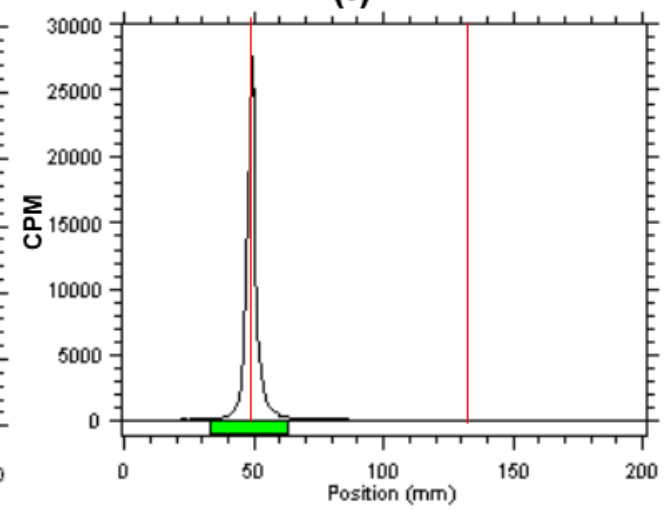

(i)

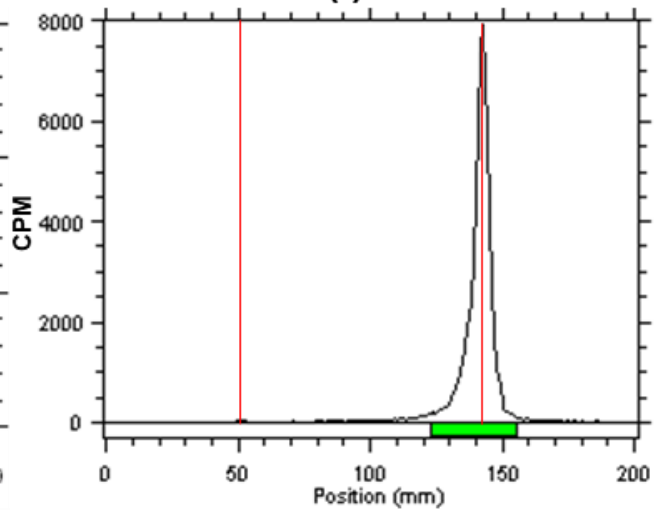

Figure S43. Radio-TLC analysis of [ $\left.{ }^{89} \mathrm{Zr}\right] \mathrm{Zr}$-PCTA prepared by using different buffers at $24^{\circ} \mathrm{C}$ or $37^{\circ} \mathrm{C}$ for 60-120 min. (a) $1 \mathrm{M} \mathrm{NH}_{4} \mathrm{OAc}, 37^{\circ} \mathrm{C}, 60 \mathrm{~min}$; (b) $1 \mathrm{M} \mathrm{NaOAc}, 37^{\circ} \mathrm{C}, 60 \mathrm{~min}$; (c) $1 \mathrm{M} \mathrm{TRIS}, 37^{\circ} \mathrm{C}, 60 \mathrm{~min}$; (d) $1 \mathrm{M}$ TMAA, $37^{\circ} \mathrm{C}, 60 \mathrm{~min}$; (e) $0.5 \mathrm{M} \mathrm{MES}, 37^{\circ} \mathrm{C}, 60 \mathrm{~min}$; (f) $0.5 \mathrm{M} \mathrm{HEPES}, 37^{\circ} \mathrm{C}, 60 \mathrm{~min}$; (g) $0.5 \mathrm{M} \mathrm{HEPES}$, $24{ }^{\circ} \mathrm{C}, 60 \mathrm{~min}$; (h) $0.5 \mathrm{M} \mathrm{HEPES}, 24^{\circ} \mathrm{C}, 120 \mathrm{~min}$; (i) $\left[{ }^{89} \mathrm{Zr}\right] \mathrm{ZrCl}$. In this ITLC-SA system, free ${ }^{89} \mathrm{Zr}$ formed a complex with EDTA and eluted with the solvent front $\left(\mathrm{R}_{\mathrm{f}} \sim 1\right)$, while $\left[{ }^{89} \mathrm{Zr}\right] \mathrm{Zr}-\mathrm{PCTA}$ complex remained at the origin $\left(\mathrm{R}_{\mathrm{f}} \sim 0\right)$. 
(a)

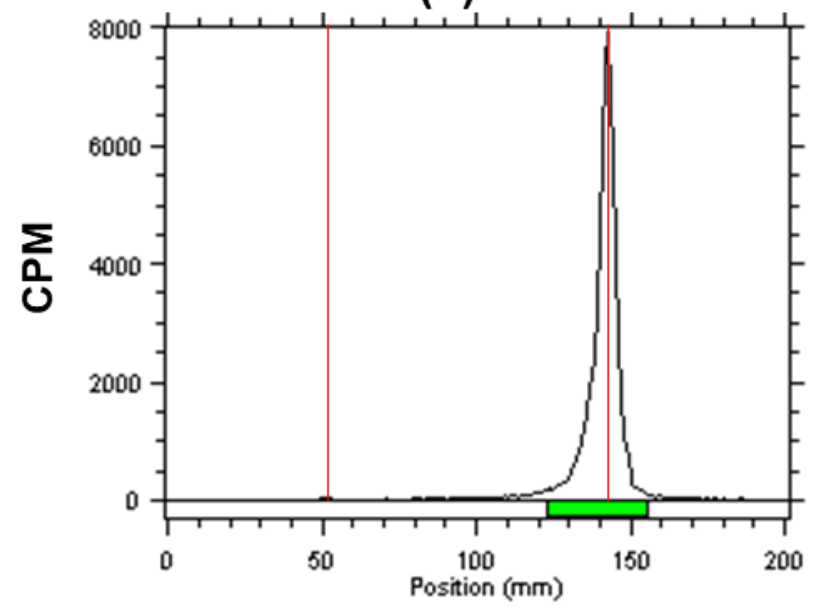

(b)

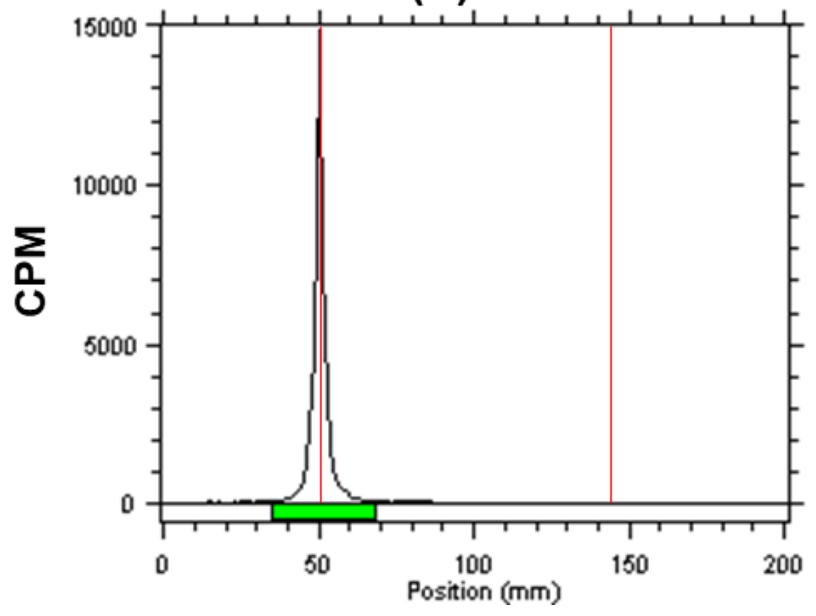

Figure S44. Quality control of $\left[{ }^{89} \mathrm{Zr}\right] \mathrm{Zr}$-PCTA by radio-TLC. ITLC-SA of $\left.{ }^{89} \mathrm{Zr}\right] \mathrm{ZrCl}{ }_{4}(\mathrm{a}),\left[{ }^{89} \mathrm{Zr}\right] \mathrm{Zr}$-PCTA (b) 


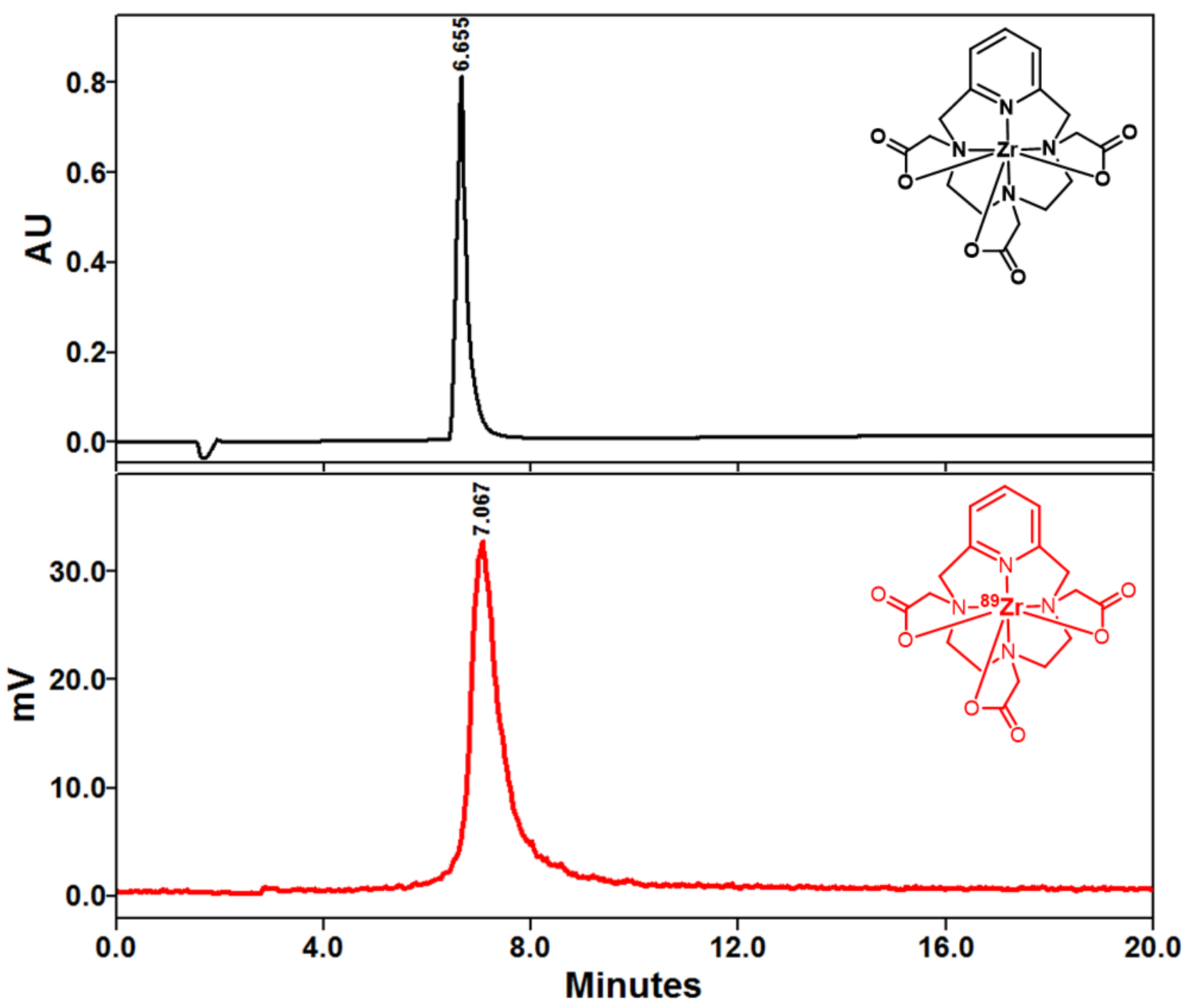

Figure S45. Quality control of [ $\left.{ }^{89} \mathrm{Zr}\right] \mathrm{Zr}-\mathrm{PCTA}$ by radio-HPLC. UV-HPLC chromatogram (201 $\left.\mathrm{nm}\right)$ of nonradioactive NatZr-PCTA complex (top) compared with radio-HPLC chromatogram of [ $\left.{ }^{89} \mathrm{Zr}\right] \mathrm{Zr}$-PCTA (bottom) 
Radiochemical Synthesis of $\left[{ }^{89} \mathrm{Zr}\right] \mathrm{Zr}-\mathrm{NOTA}$ with $\left[{ }^{89} \mathrm{Zr}\right] \mathrm{ZrCl}_{4}$ :

Table S18. Summary of optimized radiochemistry conditions and $\left[{ }^{89} \mathrm{Zr}\right] \mathrm{ZrCl}_{4}$ activity used to prepare $\left[{ }^{89} \mathrm{Zr}\right] \mathrm{Zr}-\mathrm{NOTA}{ }^{\mathrm{a}}$ complex $(\mathrm{n}=4$ for each buffer)

\begin{tabular}{|c|c|c|c|c|c|}
\hline $\begin{array}{l}\text { Quantity of } \\
\text { Ligand }\end{array}$ & $\begin{array}{c}{\left[{ }^{89} \mathrm{Zr}\right] \mathrm{ZrCl}_{4}} \\
\text { added }(\mathrm{MBq})\end{array}$ & $\begin{array}{c}\text { Reaction Buffer } \\
\text { (pH 6.8-7.2) }\end{array}$ & $\begin{array}{c}\text { Reaction } \\
\text { Temperature } \\
\left({ }^{\circ} \mathrm{C}\right)\end{array}$ & $\begin{array}{l}\text { Reaction } \\
\text { Time (min) }\end{array}$ & $\begin{array}{l}\text { Radiochemical Yield } \\
\text { by Radio-ITLC (\%) }\end{array}$ \\
\hline \multirow{8}{*}{$\begin{array}{c}\text { NOTA } \\
(7-10 \mu \mathrm{g})\end{array}$} & 18.1 & $1 \mathrm{M} \mathrm{NH}_{4} \mathrm{OAC}$ & 37 & 60 & $62.7 \pm 1.2$ \\
\hline & 18.4 & $1 \mathrm{M} \mathrm{NaOAc}$ & 37 & 60 & $30.2 \pm 1.8$ \\
\hline & 17.9 & $1 \mathrm{M}$ TRIS & 37 & 60 & $20.5 \pm 1.5$ \\
\hline & 18.0 & $1 \mathrm{M}$ TMAA & 37 & 60 & $2.2 \pm 0.6$ \\
\hline & 18.5 & $0.5 \mathrm{M}$ MES & 37 & 60 & $74.5 \pm 0.7$ \\
\hline & 19.1 & 0.5 M HEPES & 37 & 60 & $99.8 \pm 0.2$ \\
\hline & 18.7 & $0.5 \mathrm{M}$ HEPES & 24 & 60 & $66.7 \pm 1.1$ \\
\hline & 18.9 & 0.5 M HEPES & 24 & 120 & $85.4 \pm 0.3$ \\
\hline
\end{tabular}

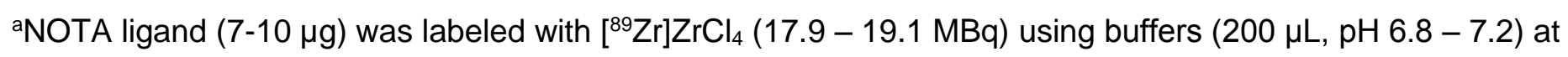
$24^{\circ} \mathrm{C}$ or $37^{\circ} \mathrm{C}$ for $60-120 \mathrm{~min}$. 
(a)

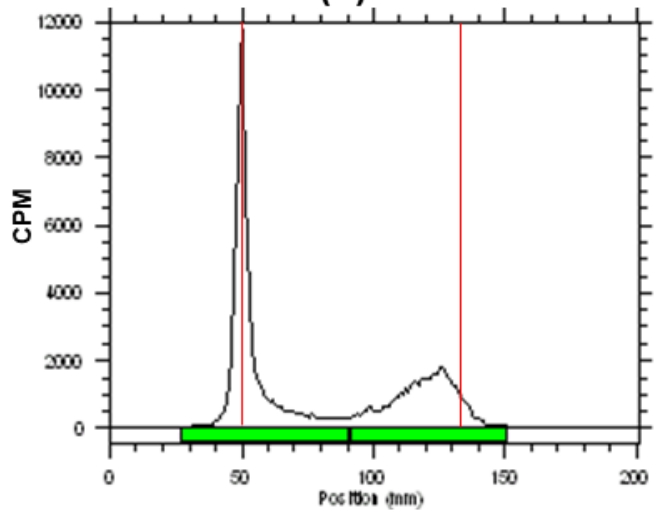

(d)

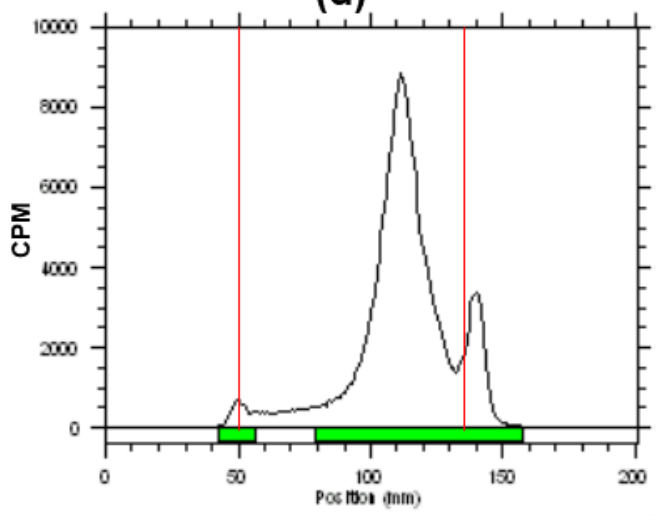

(g)

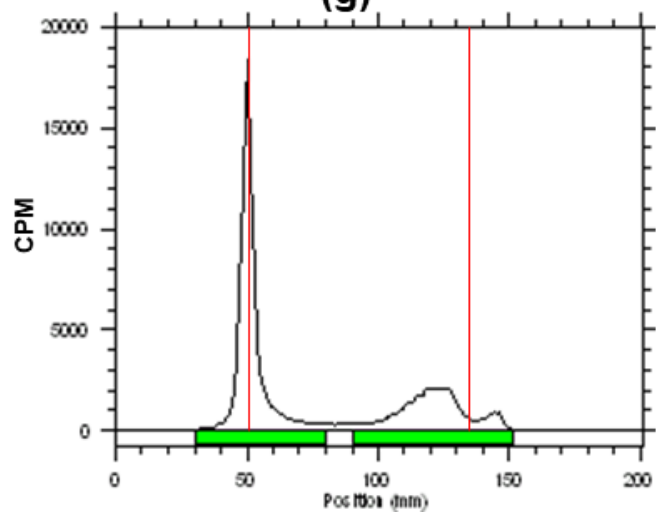

(b)

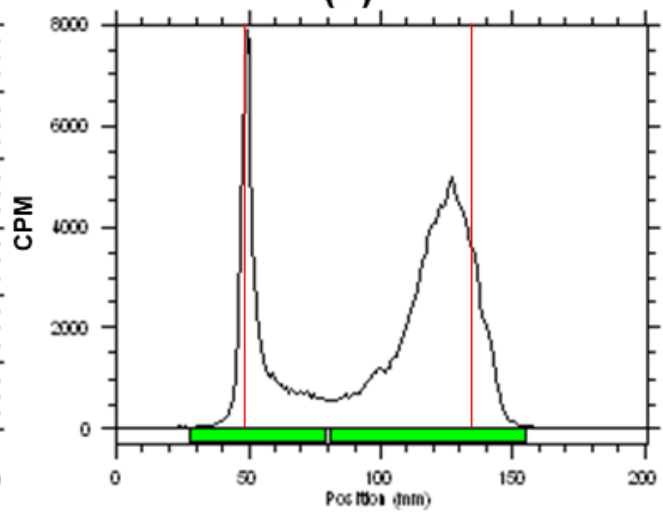

(e)

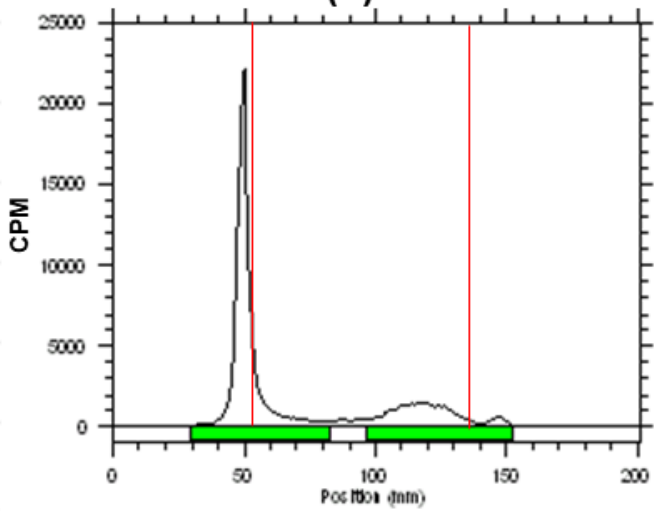

(h)

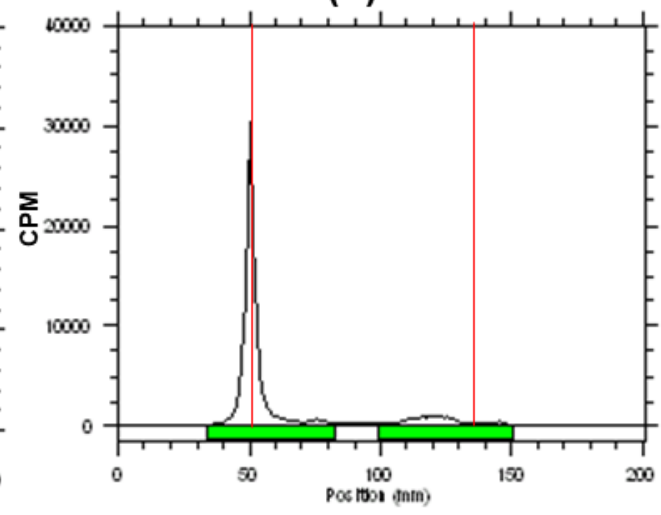

(c)

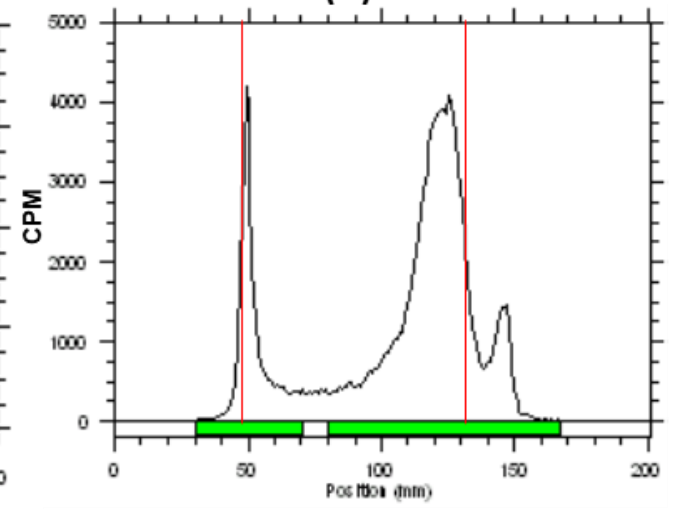

(f)

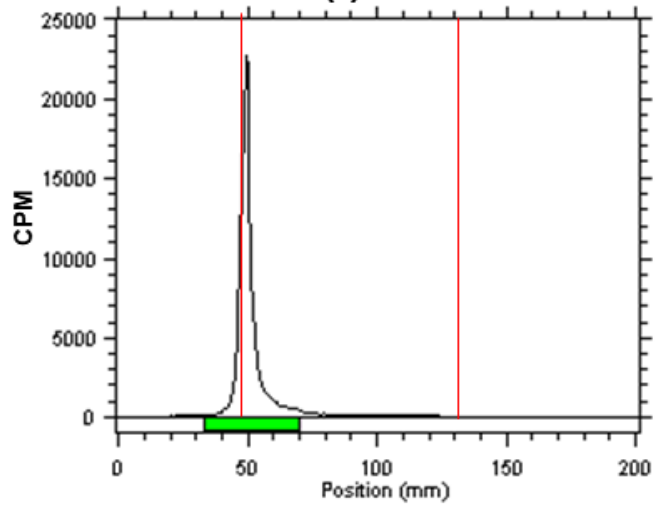

(i)

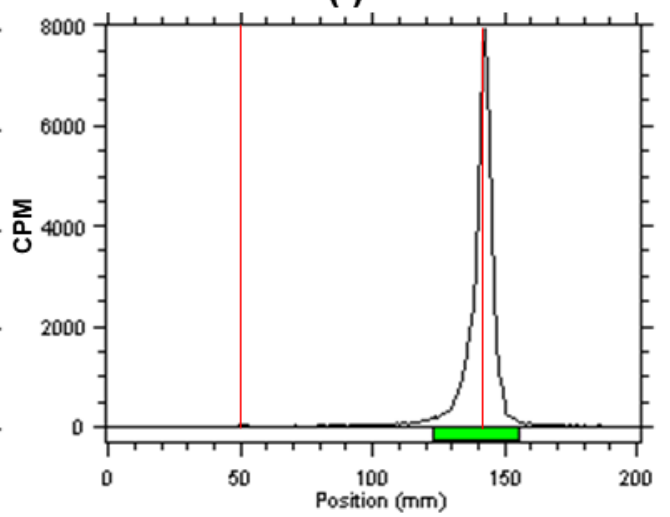

Figure S46. Radio-TLC analysis of [ $\left.{ }^{89} \mathrm{Zr}\right] \mathrm{Zr}$-NOTA prepared by using different buffers at $24^{\circ} \mathrm{C}$ or $37^{\circ} \mathrm{C}$ for 60-120 min. (a) $1 \mathrm{M} \mathrm{NH}_{4} \mathrm{OAc}, 37^{\circ} \mathrm{C}, 60 \mathrm{~min}$; (b) $1 \mathrm{M} \mathrm{NaOAc}, 37^{\circ} \mathrm{C}, 60 \mathrm{~min}$; (c) $1 \mathrm{M} \mathrm{TRIS}, 37^{\circ} \mathrm{C}, 60 \mathrm{~min}$; (d) $1 \mathrm{M}$ TMAA, $37^{\circ} \mathrm{C}, 60 \mathrm{~min}$; (e) $0.5 \mathrm{M} \mathrm{MES}, 37^{\circ} \mathrm{C}, 60 \mathrm{~min}$; (f) $0.5 \mathrm{M} \mathrm{HEPES}, 37^{\circ} \mathrm{C}, 60 \mathrm{~min}$; (g) $0.5 \mathrm{M} \mathrm{HEPES}$, $24{ }^{\circ} \mathrm{C}, 60 \mathrm{~min}$; (h) $0.5 \mathrm{M} \mathrm{HEPES}, 24{ }^{\circ} \mathrm{C}, 120 \mathrm{~min}$; (i) $\left[{ }^{89} \mathrm{Zr}\right] \mathrm{ZrCl}$. In this ITLC-SA system, free ${ }^{89} \mathrm{Zr}$ formed a complex with EDTA and eluted with the solvent front $\left(R_{f} \sim 1\right)$, while $\left[{ }^{89} \mathrm{Zr}\right] \mathrm{Zr}-\mathrm{NOTA}$ complex remained at the origin $\left(R_{f} \sim 0\right)$. 
(a)

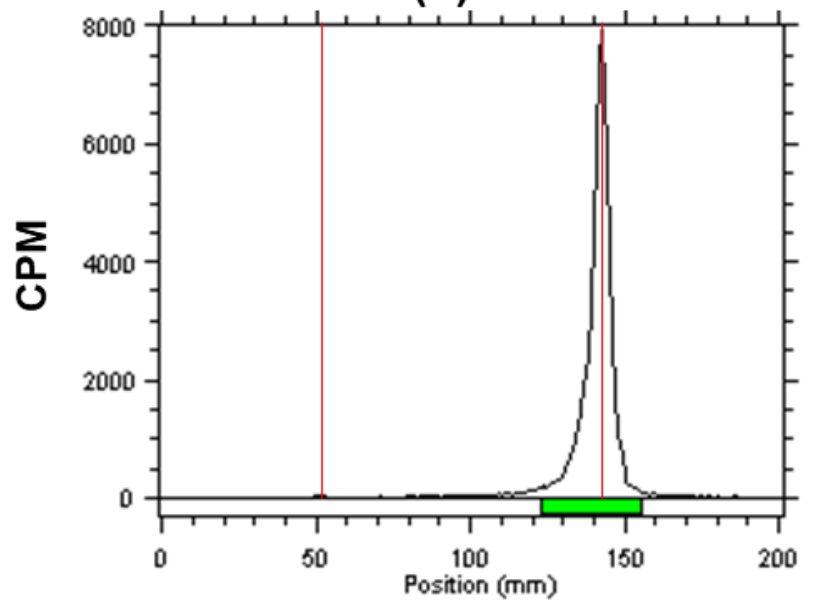

(b)

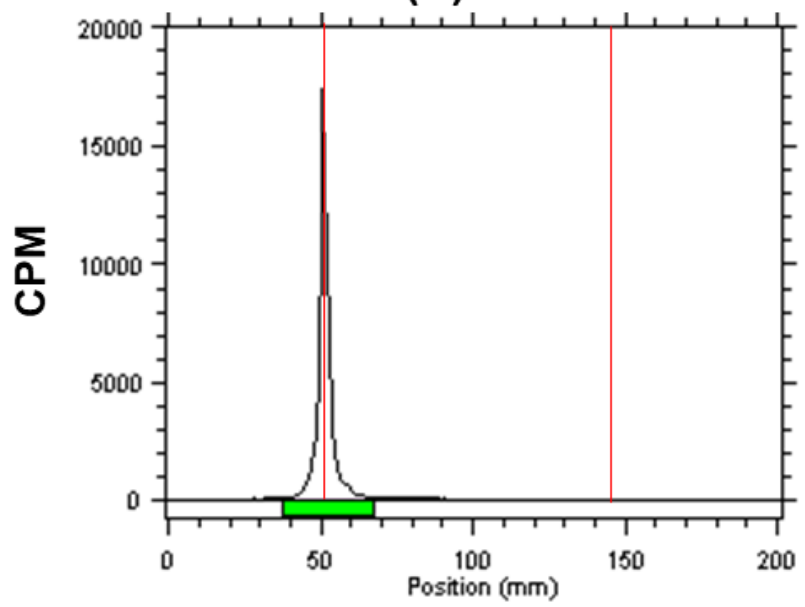

Figure S47. Quality control of [ $\left.{ }^{89} \mathrm{Zr}\right] \mathrm{Zr}-\mathrm{NOTA}$ by radio-TLC. ITLC-SA of $\left.\left[{ }^{89} \mathrm{Zr}\right] \mathrm{ZrCl}{ }_{4}(\mathrm{a}),{ }^{89} \mathrm{Zr}\right] \mathrm{Zr}-\mathrm{NOTA}$ (b) 


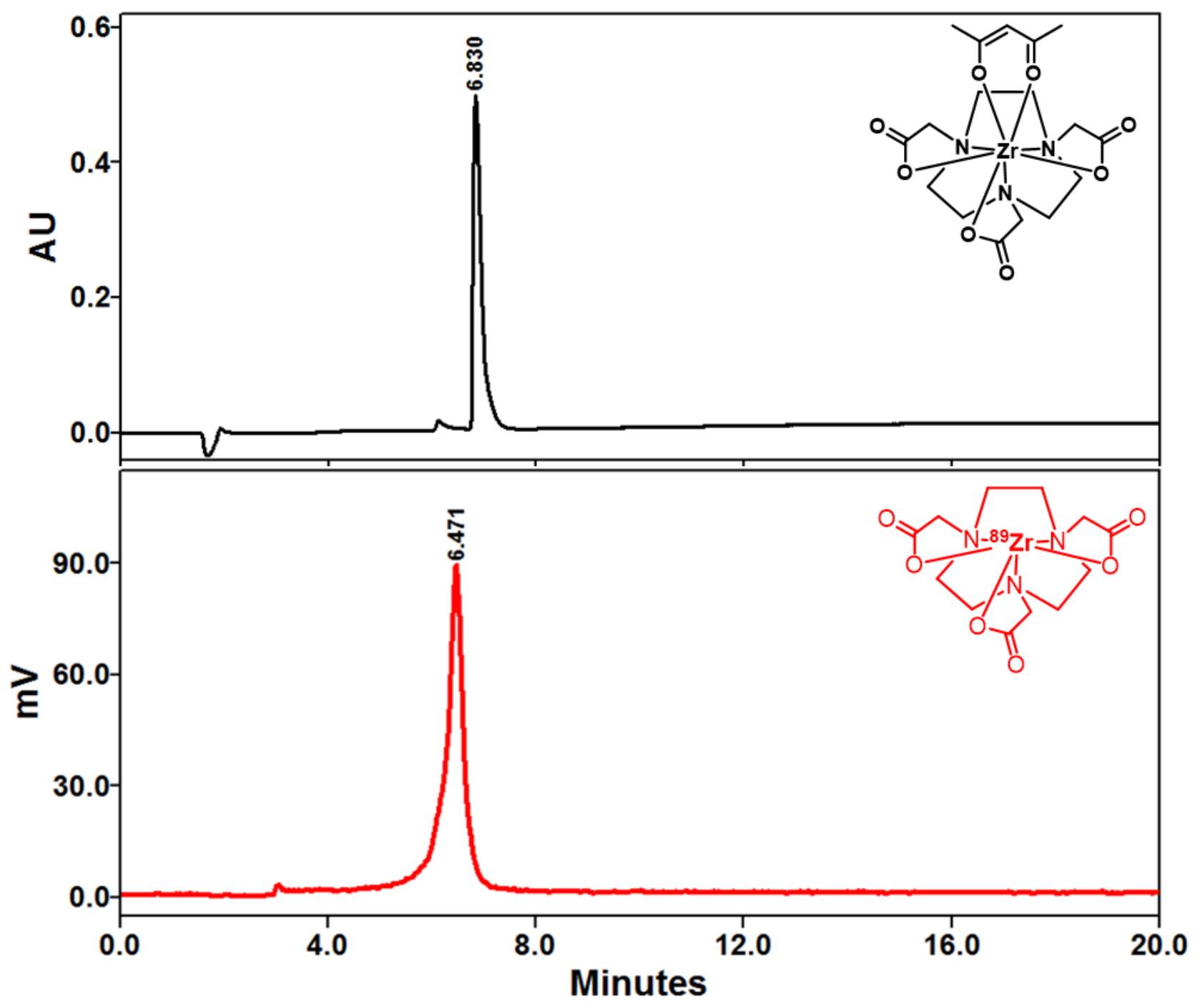

Figure S48. Quality control of $\left[{ }^{89} \mathrm{Zr}\right] \mathrm{Zr}-\mathrm{NOTA}$ by radio-HPLC. UV-HPLC chromatogram $(201 \mathrm{~nm})$ of nonradioactive NatZr-NOTA complex (top) compared with radio-HPLC chromatogram of [ $\left.{ }^{89} \mathrm{Zr}\right] \mathrm{Zr}$-NOTA (bottom) 
Radiochemical Synthesis of $\left[{ }^{89} \mathrm{Zr}\right] \mathrm{Zr}$-TRITA with [ $\left.{ }^{89} \mathrm{Zr}\right] \mathrm{ZrCl} 4$ :

Table S19. Conventional Heating: Summary of optimized radiochemistry conditions and ${ }^{89} \mathrm{Zr}^{2} \mathrm{ZrCl}_{4}$ activity used to prepare $\left.{ }^{89} \mathrm{Zr}\right] \mathrm{Zr}$-TRITA ${ }^{\mathrm{a}}$ complex $(\mathrm{n}=4$ for each buffer)

\begin{tabular}{|c|c|c|c|c|c|}
\hline $\begin{array}{l}\text { Quantity of } \\
\text { Ligand }\end{array}$ & $\begin{array}{c}{\left[{ }^{89} \mathrm{Zr}\right] \mathrm{ZrCl}_{4}} \\
\text { added }(\mathrm{MBq})\end{array}$ & $\begin{array}{l}\text { Reaction Buffer } \\
\text { (pH 6.8-7.2) }\end{array}$ & $\begin{array}{c}\text { Reaction } \\
\text { Temperature } \\
\left({ }^{\circ} \mathrm{C}\right)\end{array}$ & $\begin{array}{l}\text { Reaction } \\
\text { Time (min) }\end{array}$ & $\begin{array}{c}\text { Radiochemical Yield } \\
\text { by Radio-ITLC (\%) }\end{array}$ \\
\hline \multirow{8}{*}{$\begin{array}{c}\text { TRITA } \\
(10-15 \mu g)\end{array}$} & 17.3 & $1 \mathrm{M} \mathrm{NH}_{4} \mathrm{OAc}$ & 99 & 60 & $56.7 \pm 2.1$ \\
\hline & 17.9 & $1 \mathrm{M} \mathrm{NaOAc}$ & 99 & 60 & $43.2 \pm 1.3$ \\
\hline & 17.5 & $1 \mathrm{M}$ TRIS & 99 & 60 & $22.2 \pm 2.3$ \\
\hline & 17.1 & 1 M TMAA & 99 & 60 & $16.8 \pm 1.4$ \\
\hline & 17.4 & $0.5 \mathrm{M}$ MES & 99 & 60 & $62.9 \pm 0.9$ \\
\hline & 18.0 & $0.5 \mathrm{M}$ MES & 99 & 120 & $78.7 \pm 1.5$ \\
\hline & 17.7 & 0.5 M HEPES & 99 & 60 & $66.8 \pm 1.1$ \\
\hline & 18.1 & 0.5 M HEPES & 99 & 120 & $80.1 \pm 1.2$ \\
\hline
\end{tabular}

aTRITA ligand $(10-15 \mu \mathrm{g})$ was labeled with $\left[{ }^{89} \mathrm{Zr}\right] \mathrm{ZrCl}_{4}(17.1-18.1 \mathrm{MBq})$ using buffers $(200 \mu \mathrm{L}, \mathrm{pH} 6.8-7.2)$ at $99{ }^{\circ} \mathrm{C}$ for $60-120 \mathrm{~min}$. 
(a)

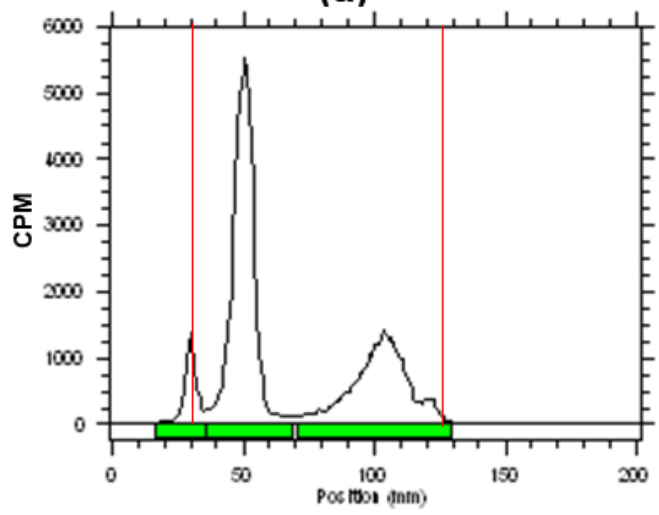

(d)

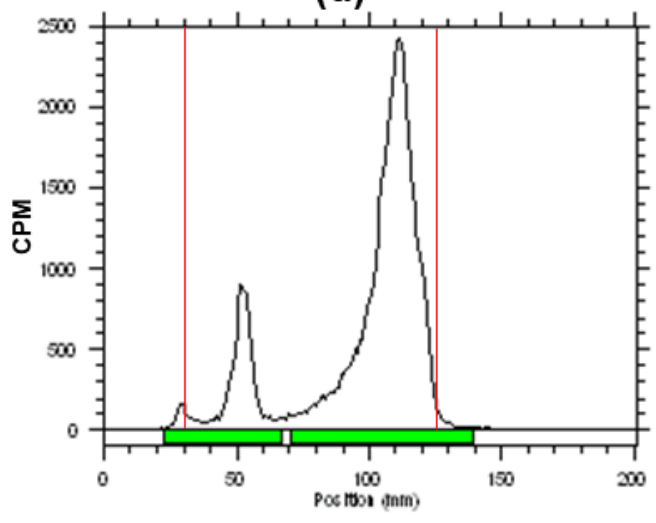

(g)

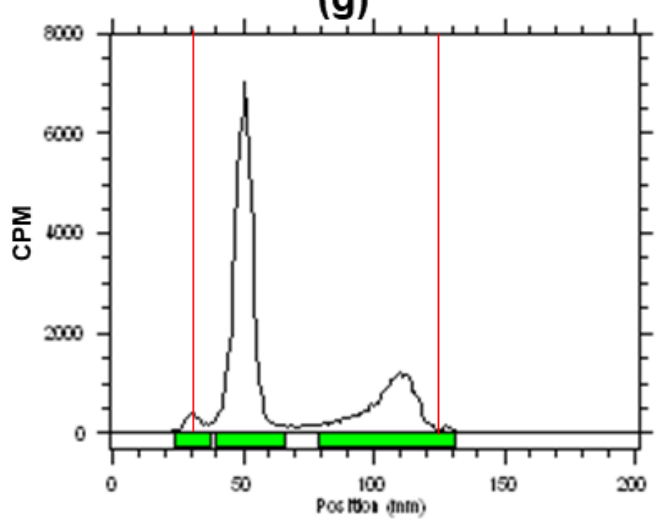

(b)

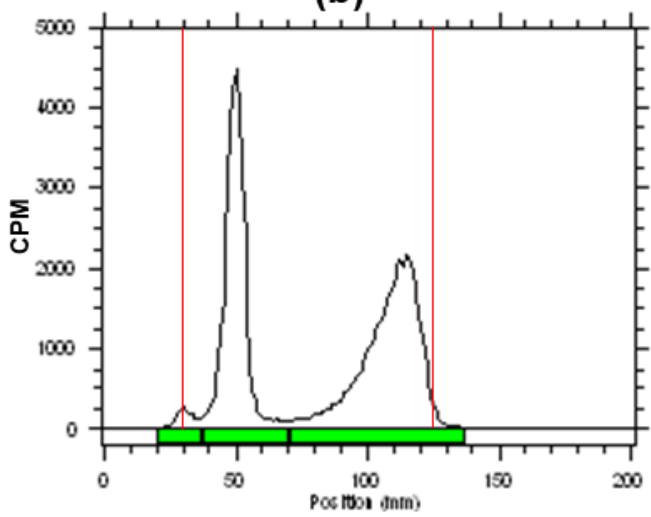

(e)

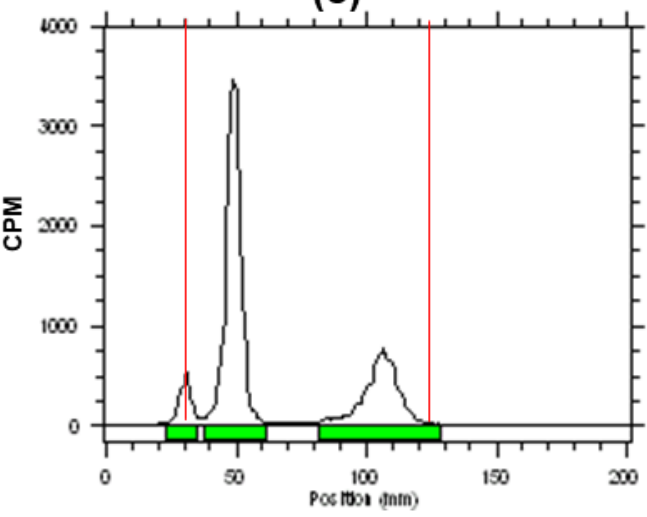

(h)

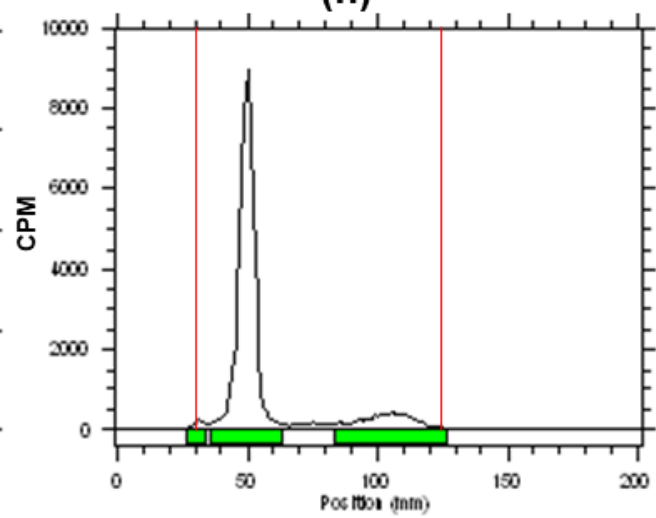

(c)

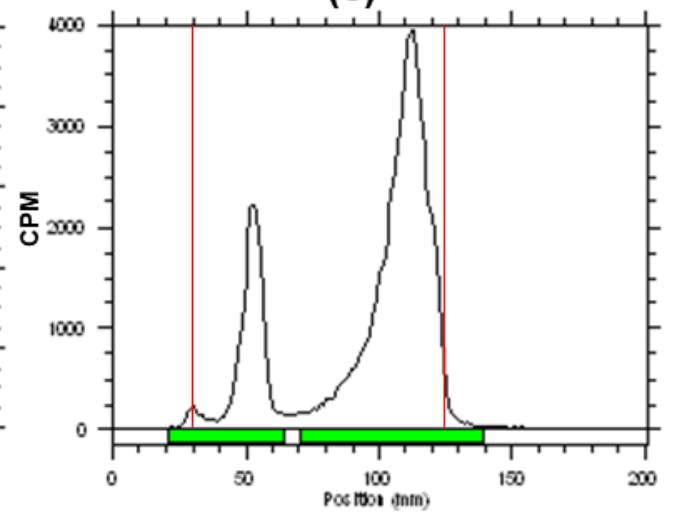

(f)

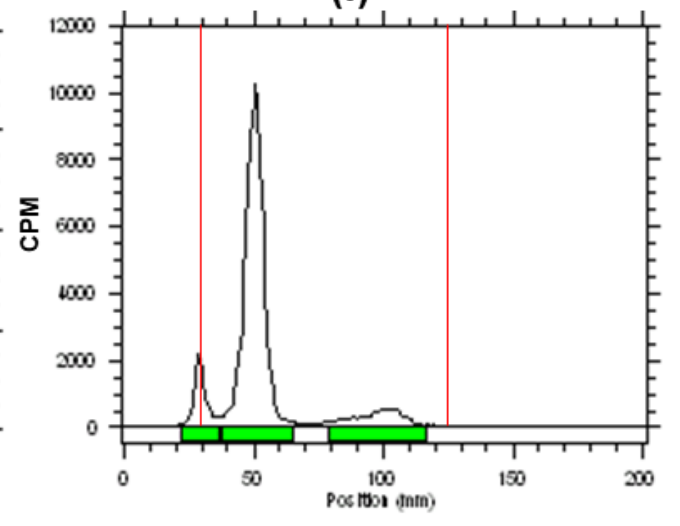

(i)

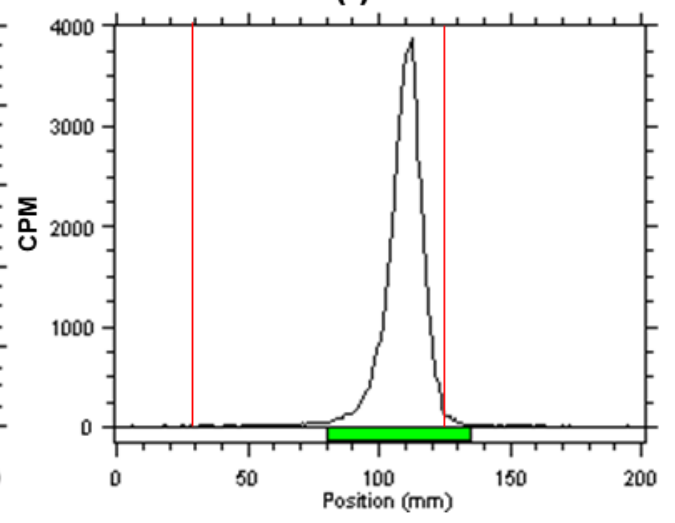

Figure S49. Conventional Heating: Radio-TLC analysis of [ ${ }^{89} \mathrm{Zr}$ ]Zr-TRITA prepared by using different buffers at $99^{\circ} \mathrm{C}$ for $\mathbf{6 0 - 1 2 0 ~ m i n . ~ ( a ) ~} 1 \mathrm{M} \mathrm{NH}_{4} \mathrm{OAc}, 99^{\circ} \mathrm{C}, 60 \mathrm{~min}$; (b) $1 \mathrm{M} \mathrm{NaOAc}, 99^{\circ} \mathrm{C}, 60 \mathrm{~min}$; (c) $1 \mathrm{M} \mathrm{TRIS}$, $99{ }^{\circ} \mathrm{C}, 60 \mathrm{~min}$; (d) $1 \mathrm{M} \mathrm{TMAA}, 99^{\circ} \mathrm{C}, 60 \mathrm{~min}$; (e) $0.5 \mathrm{M} \mathrm{MES}, 99^{\circ} \mathrm{C}, 60 \mathrm{~min}$; (f) $0.5 \mathrm{M} \mathrm{MES}, 99^{\circ} \mathrm{C}, 120 \mathrm{~min}$; (g) $0.5 \mathrm{M}$ HEPES, $99^{\circ} \mathrm{C}, 60 \mathrm{~min}$; (h) $0.5 \mathrm{M} \mathrm{HEPES}, 99^{\circ} \mathrm{C}, 120 \mathrm{~min}$; (i) $\left[{ }^{89} \mathrm{Zr}\right] \mathrm{ZrCl}$. In this ITLC-SA system, free ${ }^{89} \mathrm{Zr}$ formed a complex with EDTA and eluted with the solvent front $\left.\left(R_{f} \sim 1\right),{ }^{89} \mathrm{Zr}\right] \mathrm{Zr}$-TRITA complex moved from origin $\left(R_{f}=0.20-0.25\right)$. 
Table S20. Microwave Heating: Summary of optimized radiochemistry conditions and $\left.{ }^{89} \mathrm{Zr}\right] \mathrm{ZrCl}_{4}$ activity used to prepare $\left.{ }^{89} \mathrm{Zr}\right] \mathrm{Zr}-\mathrm{TRITA}{ }^{\mathrm{a}}$ complex ( $\mathrm{n}=4$ for each buffer)

\begin{tabular}{|c|c|c|c|c|c|}
\hline $\begin{array}{l}\text { Quantity of } \\
\text { Ligand }\end{array}$ & $\begin{array}{c}{\left[{ }^{89} \mathrm{Zr}\right] \mathrm{ZrCl}_{4}} \\
\text { added }(\mathrm{MBq})\end{array}$ & $\begin{array}{c}\text { Final Reaction } \\
\text { pH }\end{array}$ & $\begin{array}{c}\text { Reaction } \\
\text { Temperature } \\
\left({ }^{\circ} \mathrm{C}\right)\end{array}$ & $\begin{array}{l}\text { Reaction } \\
\text { Time (min) }\end{array}$ & $\begin{array}{l}\text { Radiochemical Yield } \\
\text { by Radio-ITLC (\%) }\end{array}$ \\
\hline & 15.4 & $6.8-7.2$ & 150 & 30 & $53.3 \pm 1.7$ \\
\hline & 15.2 & $5.8-6.1$ & 150 & 30 & $75.5 \pm 0.9$ \\
\hline & 15.3 & 4.9-5.1 & 150 & 30 & $89.5 \pm 0.8$ \\
\hline \multirow[t]{4}{*}{$\begin{array}{c}\text { TRITA } \\
(10-12 \mu \mathrm{g})\end{array}$} & 15.1 & $3.8-4.2$ & 150 & 30 & $93.1 \pm 0.6$ \\
\hline & 15.6 & 2.9-3.2 & 150 & 30 & $85.5 \pm 1.3$ \\
\hline & 15.7 & $3.8-4.1$ & 170 & 30 & $98.5 \pm 0.3$ \\
\hline & 15.5 & $3.9-4.2$ & 180 & 20 & $99.9 \pm 0.1$ \\
\hline
\end{tabular}

aTRITA ligand (10-12 $\mu \mathrm{g})$ was labeled with ${ }^{89} \mathrm{Zr}_{\mathrm{ZrCl}}(15.1-15.7 \mathrm{MBq})$ using $0.5 \mathrm{M}$ HEPES buffer $(50-70 \mu \mathrm{L}$, $\mathrm{pH} 7.1$-7.3) in Biotage Initiator microwave synthesizer at $150-180{ }^{\circ} \mathrm{C}$ for $20-30 \mathrm{~min}$. 
(a)

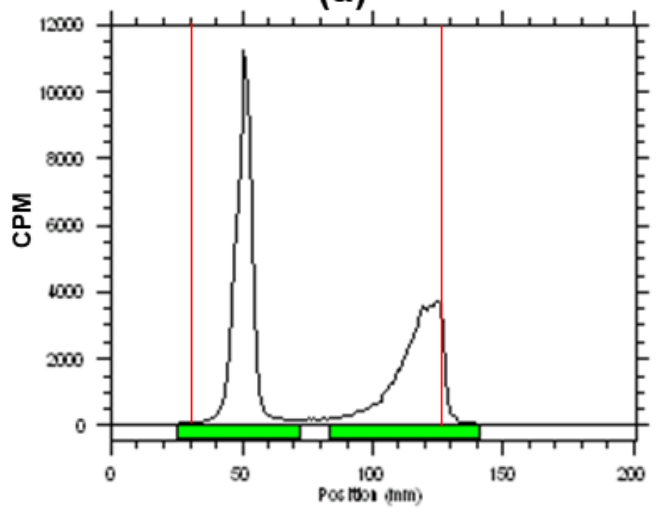

(d)

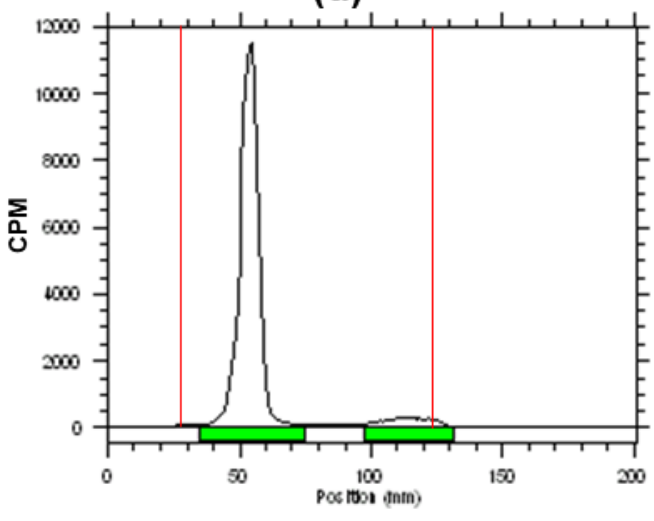

(g)

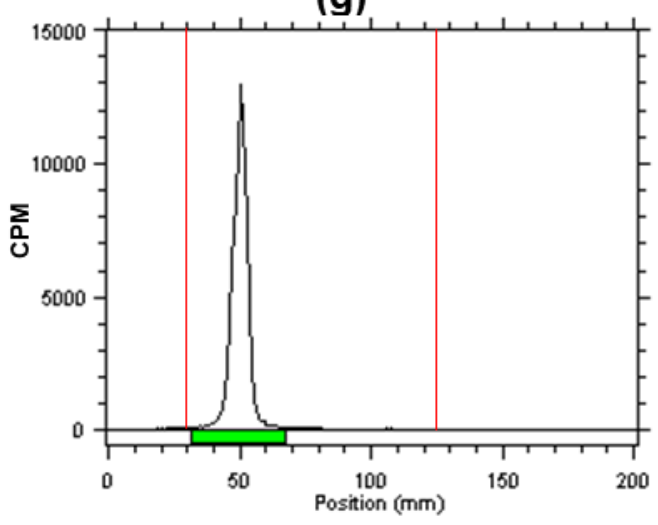

(b)

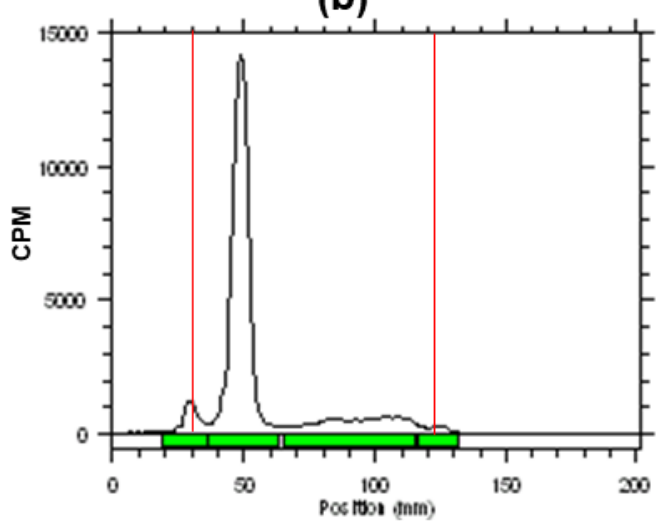

(e)

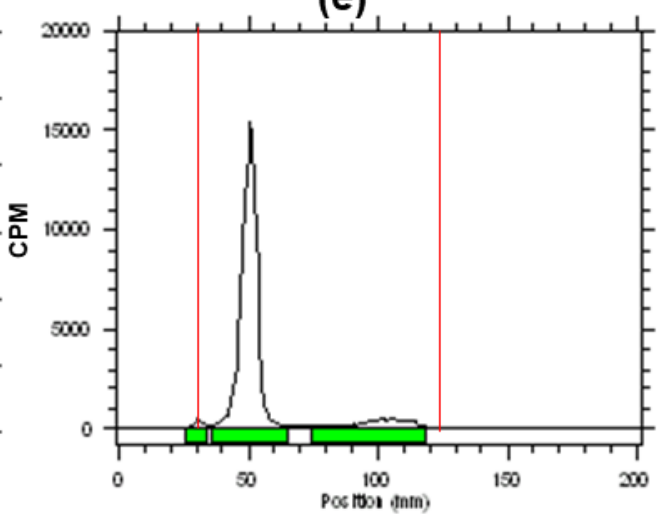

(h)

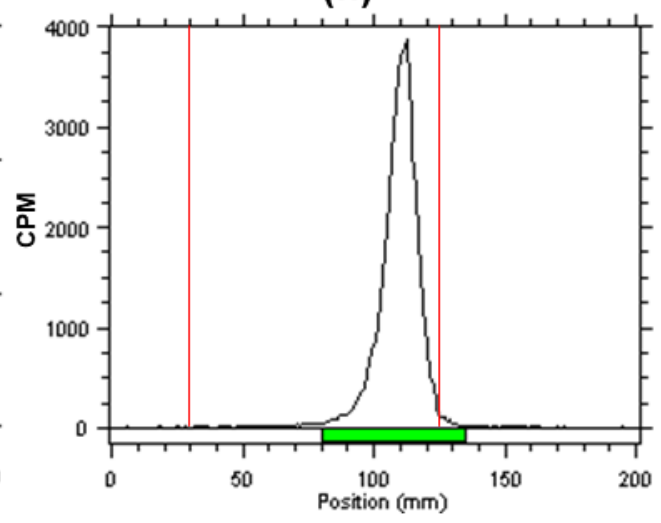

(c)

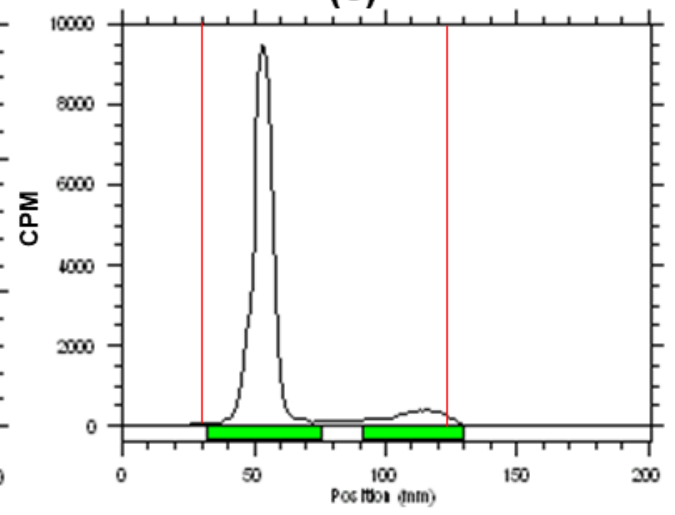

(f)

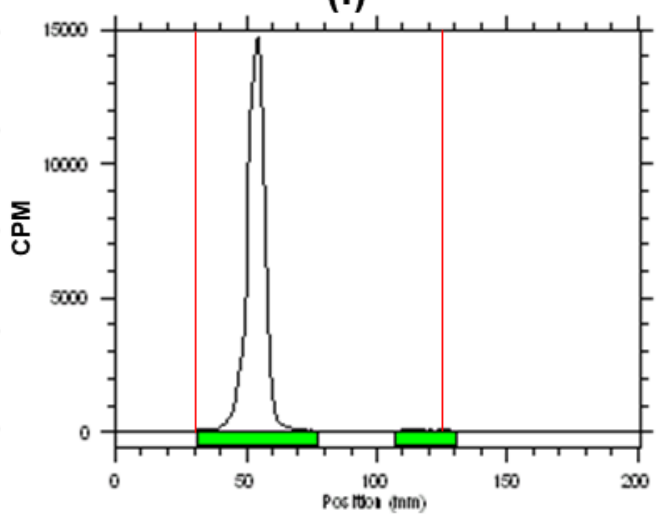

Figure S50. Microwave Heating: Radio-TLC analysis of [ $\left.{ }^{89} \mathrm{Zr}\right] \mathrm{Zr}$-TRITA prepared with different reaction pH (7.2-2.9) at 150-180 ${ }^{\circ} \mathrm{C}$ for $\mathbf{2 0 - 3 0 ~ m i n . ~ ( a ) ~ p H ~ 6 . 8 - 7 . 2 , ~} 150{ }^{\circ} \mathrm{C}, 30 \mathrm{~min}$; (b) pH 5.8-6.1, $150{ }^{\circ} \mathrm{C}, 30 \mathrm{~min}$; (c) pH 4.9-5.1, $150{ }^{\circ} \mathrm{C}, 30 \mathrm{~min}$; (d) $\mathrm{pH} 3.8-4.2,150{ }^{\circ} \mathrm{C}, 30 \mathrm{~min}$; (e) $\mathrm{pH}$ 2.9-3.2, $150{ }^{\circ} \mathrm{C}, 30 \mathrm{~min}$; (f) $\mathrm{pH} 3.8-4.1,170{ }^{\circ} \mathrm{C}$, $30 \mathrm{~min}$; (g) pH 3.9-4.2, $180{ }^{\circ} \mathrm{C}, 30 \mathrm{~min}$; (h) $\left.{ }^{89} \mathrm{Zr}\right] \mathrm{ZrCl} 4$. In this ITLC-SA system, free ${ }^{89} \mathrm{Zr}$ formed a complex with EDTA and eluted with the solvent front $\left(R_{f} \sim 1\right),\left[{ }^{89} \mathrm{Zr}\right] \mathrm{Zr}$-TRITA complex moved from origin $\left(R_{f}=0.20-0.25\right)$. 
(a)

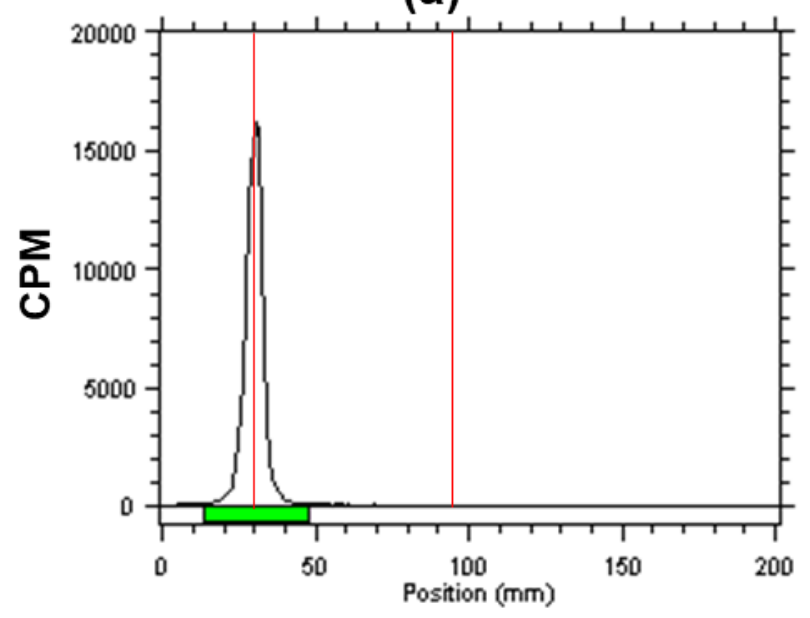

(c)

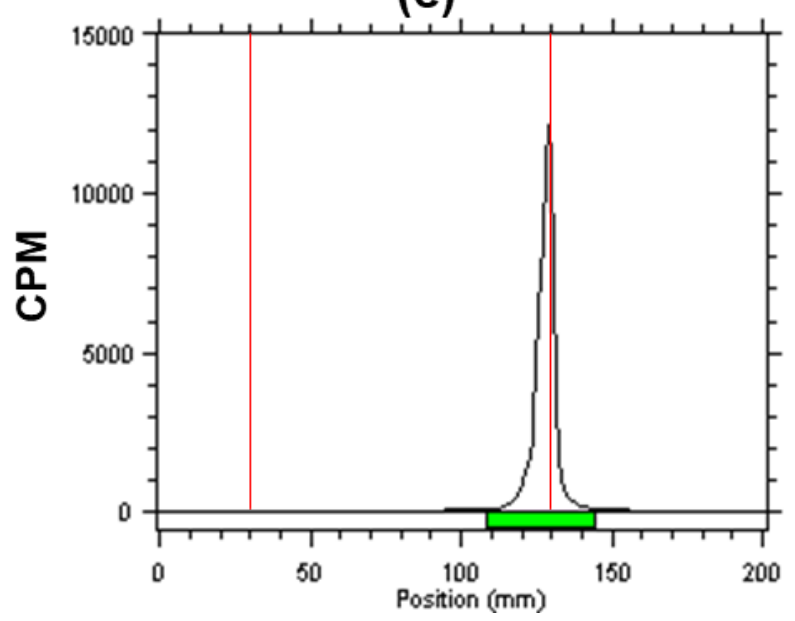

(b)

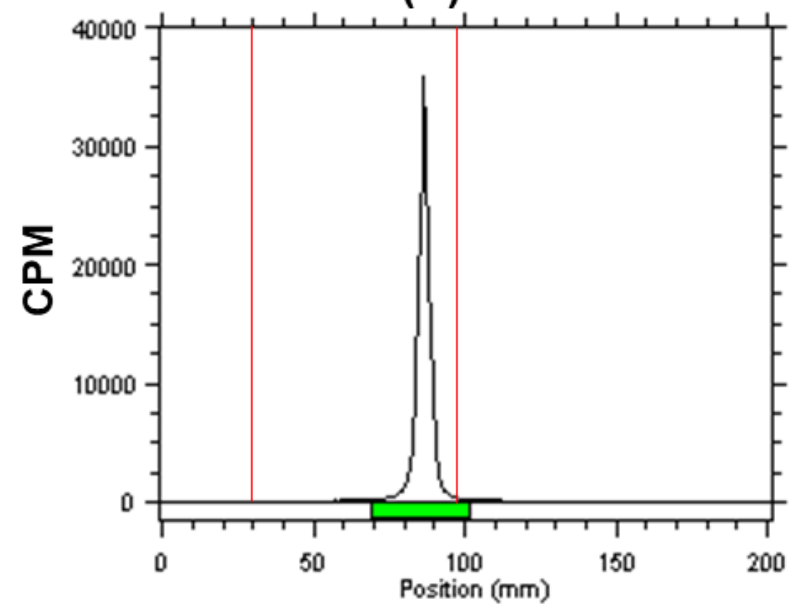

(d)

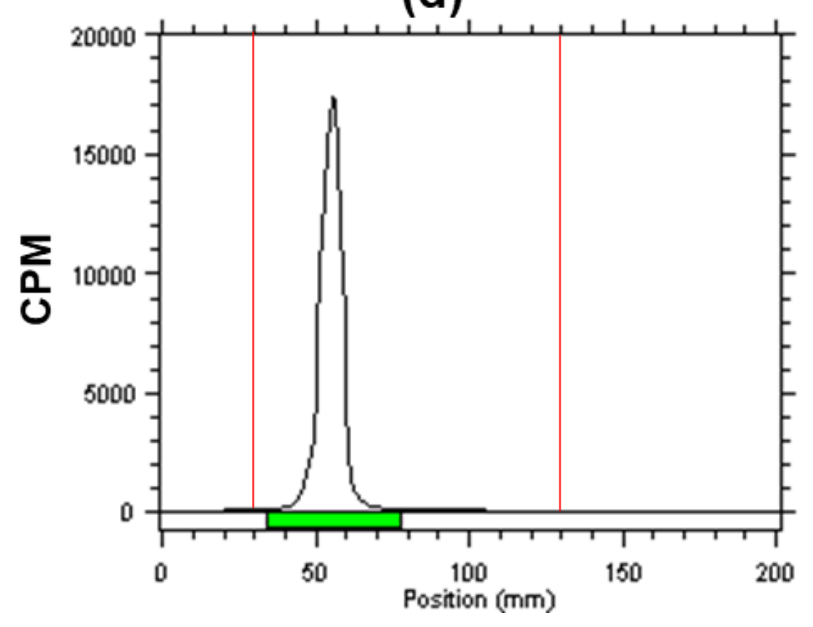

Figure S51. Quality control of $\left[{ }^{89} \mathrm{Zr}\right] \mathrm{Zr}$-TRITA by radio-TLC. C-18-TLC of $\left.{ }^{89} \mathrm{Zr}\right] \mathrm{ZrCl} \mathrm{l}_{4}(\mathrm{a}),\left[{ }^{89} \mathrm{Zr}\right] \mathrm{Zr}-\mathrm{TRITA}(\mathrm{b})$, and ITLC-SA of [ $\left.{ }^{89} \mathrm{Zr}\right] \mathrm{ZrCl}_{4}$ (c), $\left.{ }^{89} \mathrm{Zr}\right] \mathrm{Zr}-\mathrm{TRITA}$ (d) 


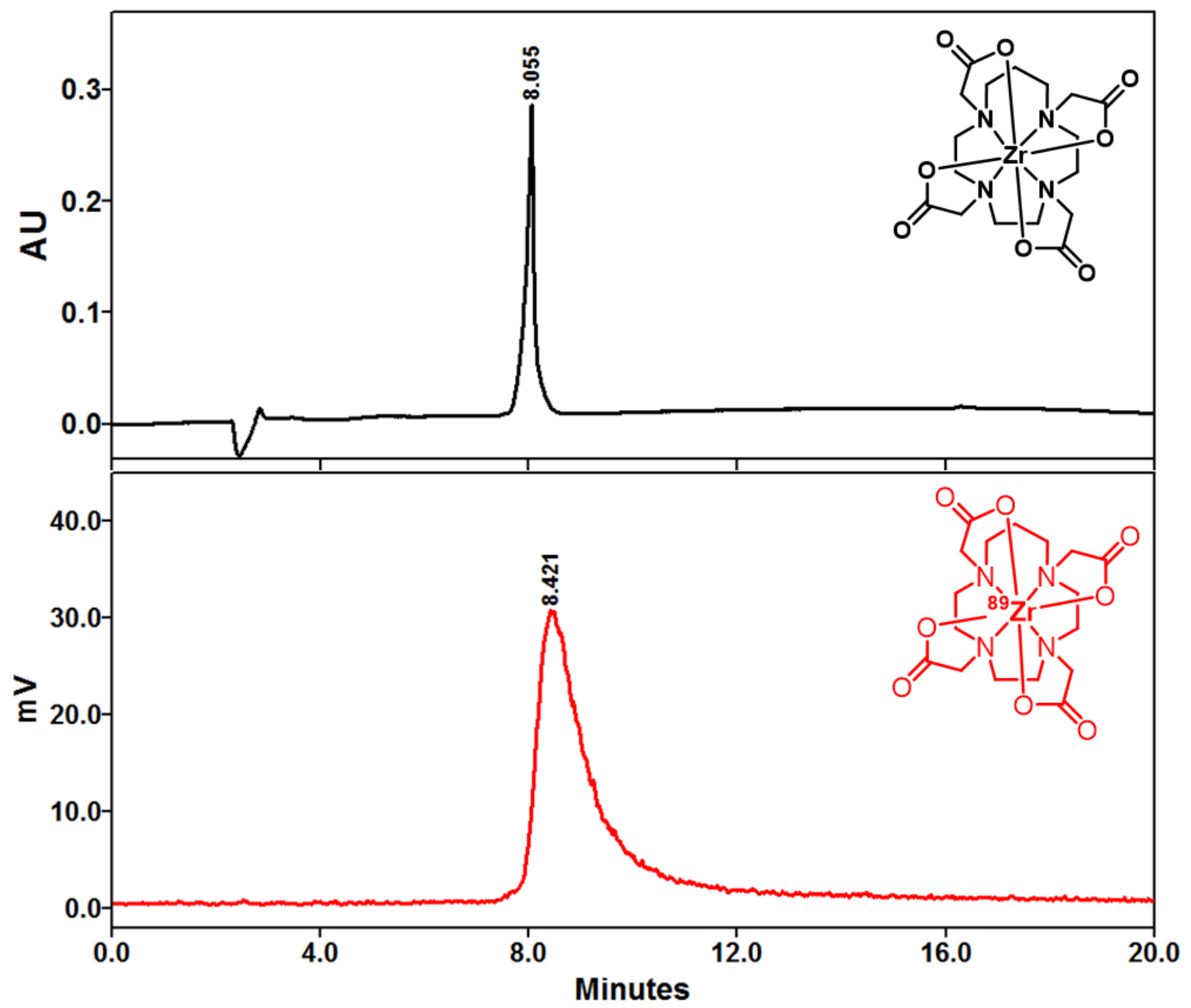

Figure S52. Quality control of [ ${ }^{89} \mathrm{Zr}$ ]Zr-TRITA by radio-HPLC. UV-HPLC chromatogram $(201 \mathrm{~nm})$ of nonradioactive ${ }^{\mathrm{Nat} Z r-T R I T A}$ complex (top) compared with radio-HPLC chromatogram of $\left[{ }^{89} \mathrm{Zr}\right] \mathrm{Zr}$-TRITA (bottom) 
Radiochemical Synthesis of $\left[{ }^{89} \mathrm{Zr}\right] \mathrm{Zr}-\mathrm{TETA}$ with $\left.{ }^{89} \mathrm{Zr}\right] \mathrm{ZrCl}_{4}$ :

Table S21. Summary of optimized radiochemistry conditions and $\left[{ }^{89} \mathrm{Zr}\right] \mathrm{ZrCl}_{4}$ activity used to prepare $\left[{ }^{89} \mathrm{Zr}\right] \mathrm{Zr}-\mathrm{TETA}{ }^{\mathrm{a}}$ complex $(\mathrm{n}=4$ for each buffer)

\begin{tabular}{|c|c|c|c|c|c|}
\hline $\begin{array}{l}\text { Quantity of } \\
\text { Ligand }\end{array}$ & $\begin{array}{c}{\left[{ }^{89} \mathrm{Zr}\right] \mathrm{ZrCl}_{4}} \\
\text { added }(\mathrm{MBq})\end{array}$ & $\begin{array}{l}\text { Reaction Buffer } \\
\text { (pH 6.8 - 7.2) }\end{array}$ & $\begin{array}{c}\text { Reaction } \\
\text { Temperature } \\
\left({ }^{\circ} \mathrm{C}\right)\end{array}$ & $\begin{array}{l}\text { Reaction } \\
\text { Time (min) }\end{array}$ & $\begin{array}{c}\text { Radiochemical Yield } \\
\text { by Radio-ITLC (\%) }\end{array}$ \\
\hline \multirow{6}{*}{$\begin{array}{c}\text { TETA } \\
(10-50 \mu \mathrm{g})\end{array}$} & 16.6 & $1 \mathrm{M} \mathrm{NH}_{4} \mathrm{OAc}$ & 99 & 120 & 0 \\
\hline & 16.8 & $1 \mathrm{M} \mathrm{NaOAc}$ & 99 & 120 & 0 \\
\hline & 17.5 & $1 \mathrm{M}$ TRIS & 99 & 120 & 0 \\
\hline & 19.2 & $0.5 \mathrm{M}$ MES & 99 & 120 & 0 \\
\hline & 18.7 & $0.5 \mathrm{M}$ HEPES & 99 & 120 & $0.5 \pm 0.1$ \\
\hline & 20.5 & $0.5 \mathrm{M}$ HEPES & $180^{\mathrm{b}}$ & 30 & $1.5 \pm 0.3$ \\
\hline
\end{tabular}

aTETA ligand (10-50 $\mu \mathrm{g})$ was labeled with $\left.{ }^{89} \mathrm{Zr}\right] \mathrm{ZrCl}_{4}(16.6-20.5 \mathrm{MBq})$ using buffers $(200 \mu \mathrm{L}, \mathrm{pH} 6.8-7.2)$ at $99^{\circ} \mathrm{C}$ for $120 \mathrm{~min}$.

${ }^{\mathrm{b} T E T A}$ ligand $(10-50 \mu \mathrm{g})$ was labeled with ${ }^{89} \mathrm{Zr}_{\mathrm{ZrCl}}(20.5 \mathrm{MBq})$ using $0.5 \mathrm{M} \mathrm{HEPES}$ buffer $(50-70 \mu \mathrm{L}, \mathrm{pH} 7.1$ 7.3) in Biotage Initiator microwave synthesizer at $180^{\circ} \mathrm{C}$ for $30 \mathrm{~min}$. 
(a)

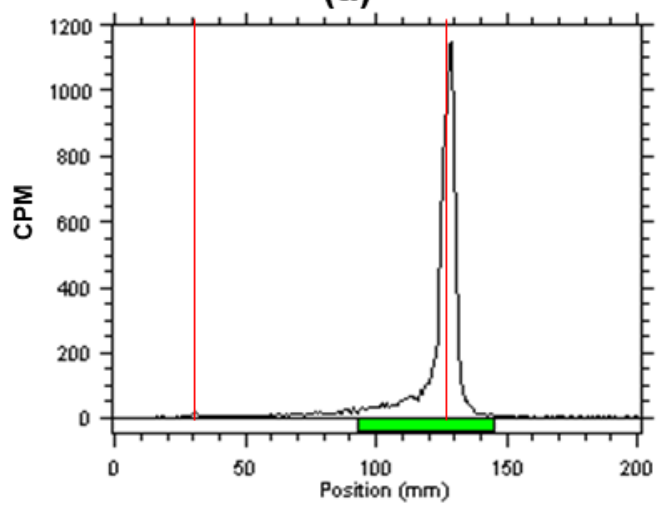

(d)

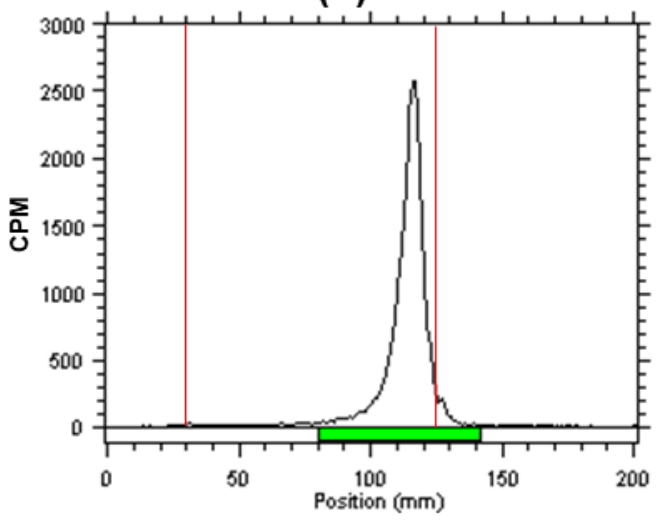

(g)

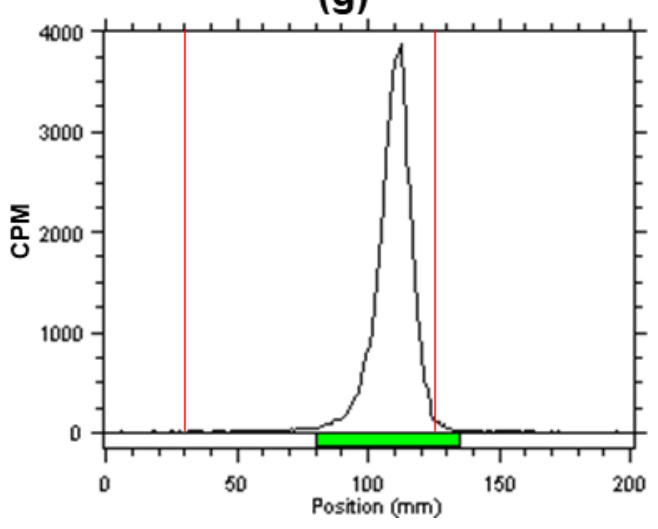

(b)

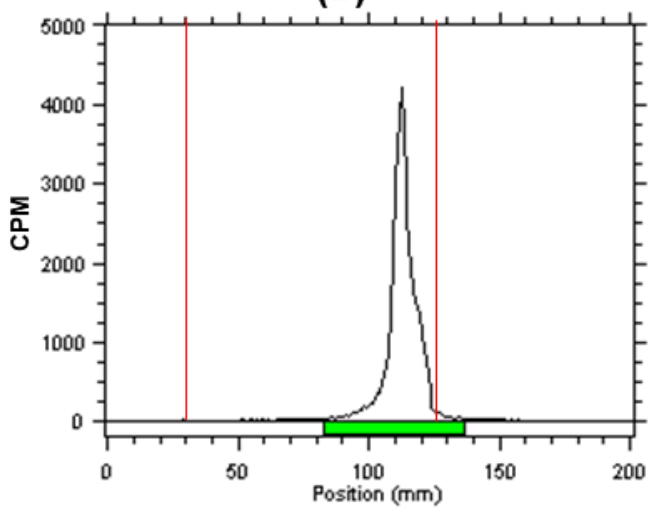

(e)

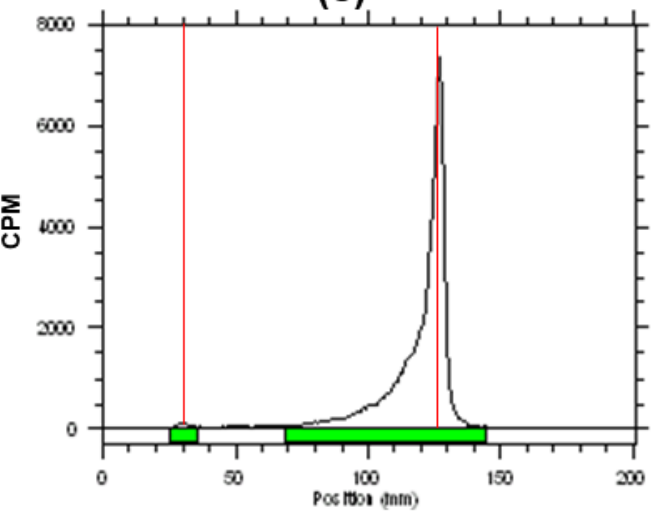

(c)

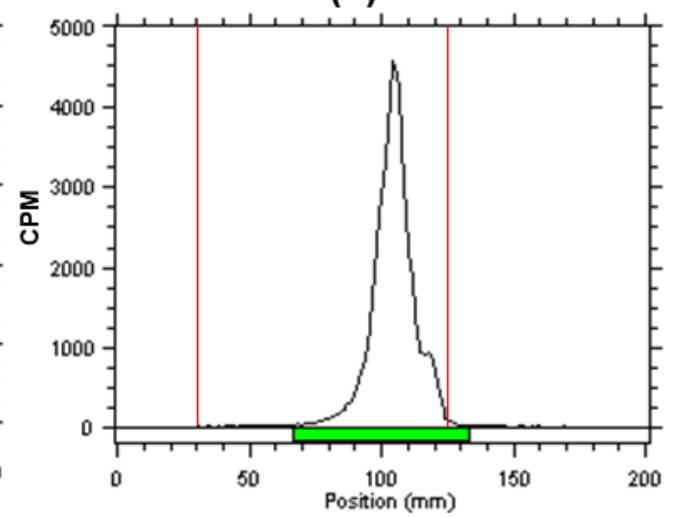

(f)

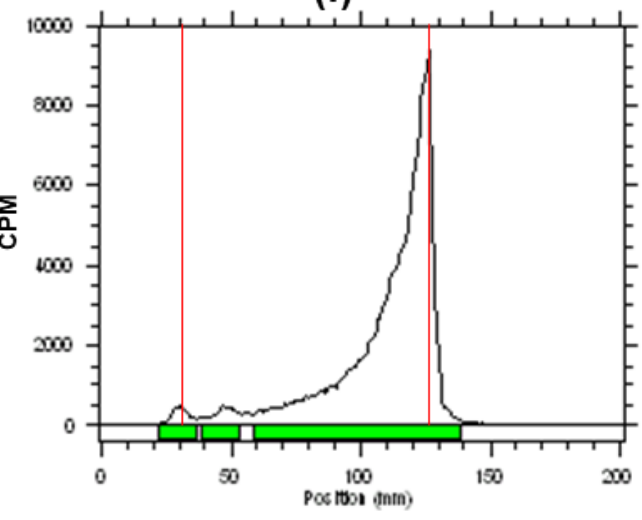

Figure S53. Radio-TLC analysis of [ $\left.{ }^{89} \mathrm{Zr}\right] \mathrm{Zr}$-TETA prepared by using different buffers at $99{ }^{\circ} \mathrm{C}$ for $120 \mathrm{~min}$. (a) $1 \mathrm{M} \mathrm{NH}_{4} \mathrm{OAc}$; (b) $1 \mathrm{M} \mathrm{NaOAc}$; (c) $1 \mathrm{M}$ TRIS; (d) $0.5 \mathrm{M} \mathrm{MES}$; (e) $0.5 \mathrm{M}$ HEPS (Conventional heating); (f) 0.5 M HEPES (Microwave heating); (g) [ $\left.{ }^{89} \mathrm{Zr}\right] \mathrm{ZrCl}_{4}$. In this ITLC-SA system, free ${ }^{89} \mathrm{Zr}$ formed a complex with EDTA and eluted with the solvent front $\left(R_{f} \sim 1\right)$, while $\left[{ }^{89} \mathrm{Zr}\right] \mathrm{Zr}$-TETA complex remained at the origin $\left(\mathrm{R}_{\mathrm{f}} \sim 0\right)$ 
Table S22. Log $P^{7}$ values for all $\left.{ }^{89} \mathrm{Zr}\right] \mathrm{Zr}$-complexes

\begin{tabular}{cc}
\hline Complex & $\log \mathbf{P}(\mathbf{n}=5)$ \\
\hline$\left[{ }^{89} \mathrm{Zr}\right] \mathrm{Zr}-\mathrm{PCTA}$ & $-3.09 \pm 0.03$ \\
{$\left[{ }^{89} \mathrm{Zr}\right] \mathrm{Zr}-\mathrm{NOTA}$} & $-2.51 \pm 0.01$ \\
{$\left[{ }^{89} \mathrm{Zr}\right] \mathrm{Zr}-\mathrm{TRITA}$} & $-3.13 \pm 0.02$ \\
\hline
\end{tabular}



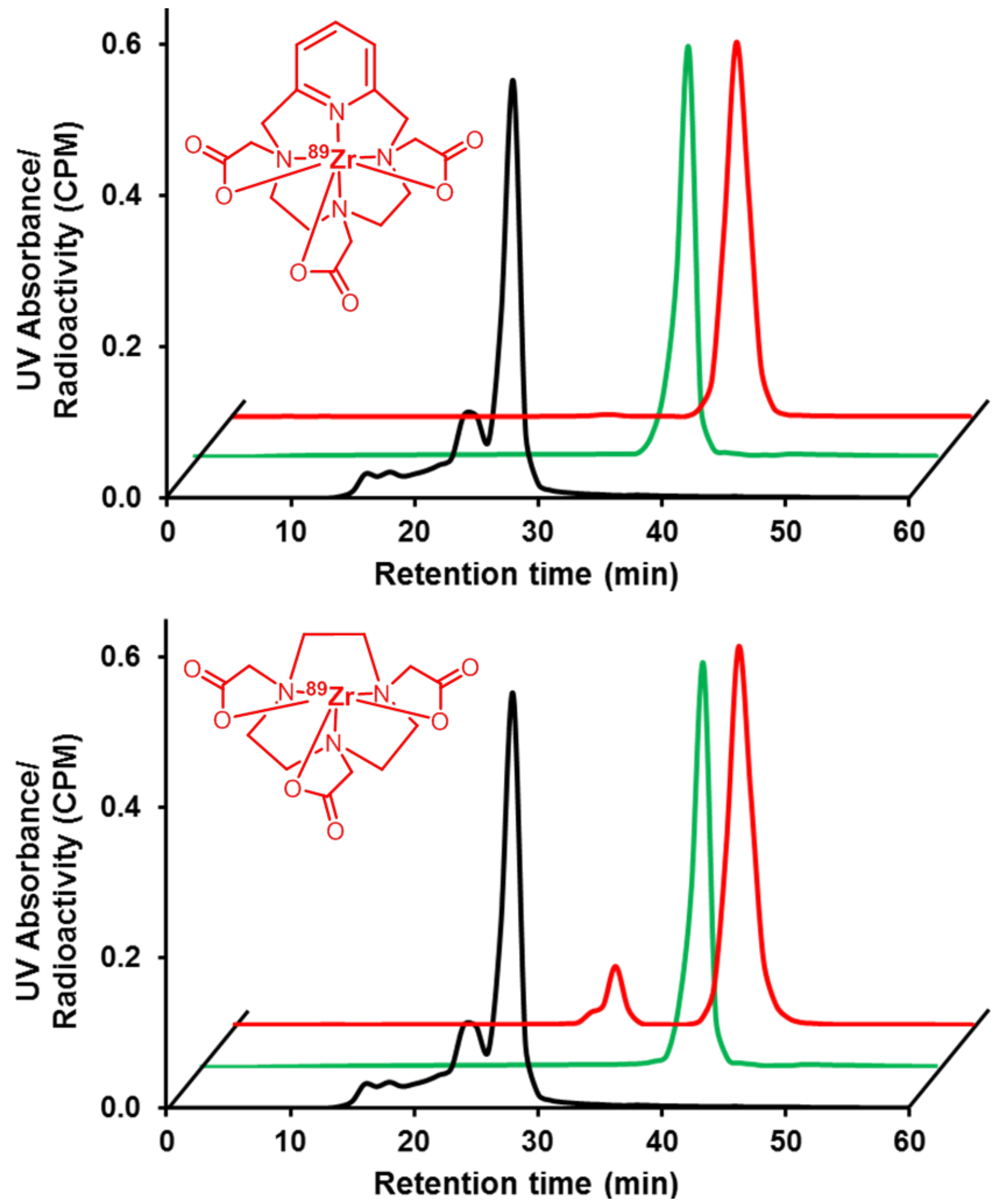

Figure S54. In vitro serum stability by SE-HPLC. UV-SE-HPLC $(220 \mathrm{~nm}$, black, and green) and radio-SEHPLC chromatogram (red) of [ $\left.{ }^{89} \mathrm{Zr}\right] \mathrm{Zr}$-PCTA (top), and $\left[{ }^{89} \mathrm{Zr}\right] \mathrm{Zr}-\mathrm{NOTA}$ (bottom) in serum after 7 days. Black lines are the UV absorbance due to the human serum components; green lines are the UV absorbance associated with ${ }^{N a t Z r-c o m p l e x e s ~ a n d ~ r e d ~ l i n e s ~ a r e ~ t h e ~ r a d i o t r a c e r ~ a s s o c i a t e d ~ w i t h ~}\left[{ }^{89} \mathrm{Zr}\right] \mathrm{Zr}$-complexes. 

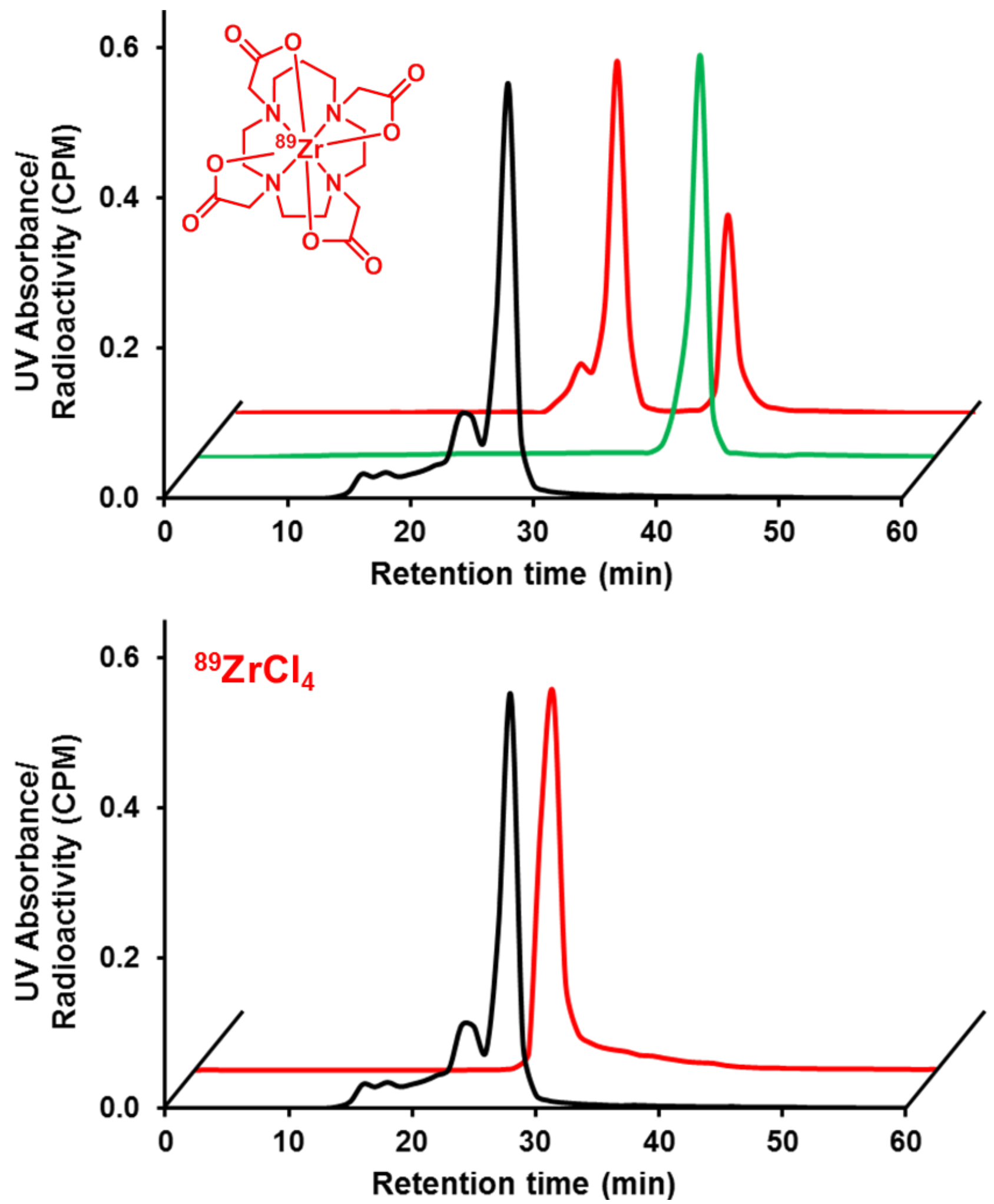

Figure S55. In vitro serum stability by SE-HPLC ${ }^{8}$. UV-SE-HPLC $(220 \mathrm{~nm}$, black, and green) and radio-SEHPLC chromatogram (red) of $\left[{ }^{89} \mathrm{Zr}\right] \mathrm{Zr}$-TRITA (top), and $\left[{ }^{89} \mathrm{Zr}\right] \mathrm{ZrCl} 4$ (bottom) in serum after 7 days. Black lines are the UV absorbance due to the human serum components; green lines are the UV absorbance associated with NatZr-complexes and red lines are the radiotracer associated with ${ }^{89} \mathrm{Zr}$-complexes. 
Table S23. Biodistribution (\%ID/g) ${ }^{9-10}$ of $\left[{ }^{89} \mathrm{Zr}\right] \mathrm{Zr}-\mathrm{PCTA}$ in selected organs at $2,4,24,48$, and $72 \mathrm{~h}$ p.i.

\begin{tabular}{|c|c|c|c|c|c|}
\hline Tissue/Organ & $2 \mathrm{~h}$ & $4 \mathrm{~h}$ & $24 \mathrm{~h}$ & $48 \mathrm{~h}$ & $72 \mathrm{~h}$ \\
\hline Blood & $0.013 \pm 0.003$ & $0.008 \pm 0.002$ & $0.002 \pm 0.001$ & $0.000 \pm 0.001$ & $0.000 \pm 0.001$ \\
\hline Heart & $0.024 \pm 0.003$ & $0.020 \pm 0.004$ & $0.010 \pm 0.003$ & $0.008 \pm 0.002$ & $0.007 \pm 0.002$ \\
\hline Lung & $0.061 \pm 0.006$ & $0.052 \pm 0.005$ & $0.026 \pm 0.003$ & $0.017 \pm 0.002$ & $0.014 \pm 0.003$ \\
\hline Liver & $0.131 \pm 0.005$ & $0.104 \pm 0.010$ & $0.056 \pm 0.003$ & $0.037 \pm 0.004$ & $0.030 \pm 0.003$ \\
\hline Small intestine & $0.122 \pm 0.023$ & $0.058 \pm 0.016$ & $0.012 \pm 0.001$ & $0.006 \pm 0.001$ & $0.004 \pm 0.000$ \\
\hline Large intestine & $0.193 \pm 0.060$ & $0.413 \pm 0.061$ & $0.037 \pm 0.008$ & $0.011 \pm 0.003$ & $0.006 \pm 0.001$ \\
\hline Kidney & $0.722 \pm 0.068$ & $0.571 \pm 0.050$ & $0.416 \pm 0.016$ & $0.272 \pm 0.031$ & $0.151 \pm 0.010$ \\
\hline Spleen & $0.047 \pm 0.007$ & $0.041 \pm 0.010$ & $0.035 \pm 0.004$ & $0.026 \pm 0.004$ & $0.024 \pm 0.003$ \\
\hline Pancreas & $0.016 \pm 0.003$ & $0.015 \pm 0.003$ & $0.009 \pm 0.002$ & $0.007 \pm 0.002$ & $0.003 \pm 0.002$ \\
\hline Stomach & $0.027 \pm 0.006$ & $0.026 \pm 0.005$ & $0.009 \pm 0.002$ & $0.004 \pm 0.001$ & $0.003 \pm 0.001$ \\
\hline Muscle & $0.013 \pm 0.004$ & $0.007 \pm 0.002$ & $0.006 \pm 0.002$ & $0.002 \pm 0.001$ & $0.001 \pm 0.001$ \\
\hline Fat & $0.012 \pm 0.001$ & $0.008 \pm 0.002$ & $0.007 \pm 0.002$ & $0.006 \pm 0.002$ & $0.002 \pm 0.002$ \\
\hline Bone & $0.104 \pm 0.015$ & $0.066 \pm 0.010$ & $0.113 \pm 0.009$ & $0.105 \pm 0.007$ & $0.094 \pm 0.006$ \\
\hline
\end{tabular}




\begin{tabular}{|c|c|c|c|c|c|}
\hline Tissue/Organ & $2 \mathrm{~h}$ & $4 \mathrm{~h}$ & $24 \mathrm{~h}$ & $48 \mathrm{~h}$ & $72 \mathrm{~h}$ \\
\hline Blood & $0.029 \pm 0.002$ & $0.014 \pm 0.002$ & $0.003 \pm 0.001$ & $0.001 \pm 0.000$ & $0.001 \pm 0.000$ \\
\hline Heart & $0.037 \pm 0.002$ & $0.027 \pm 0.003$ & $0.015 \pm 0.001$ & $0.011 \pm 0.002$ & $0.007 \pm 0.002$ \\
\hline Lung & $0.109 \pm 0.008$ & $0.068 \pm 0.004$ & $0.034 \pm 0.003$ & $0.017 \pm 0.002$ & $0.016 \pm 0.001$ \\
\hline Liver & $0.591 \pm 0.011$ & $0.450 \pm 0.025$ & $0.158 \pm 0.010$ & $0.086 \pm 0.007$ & $0.069 \pm 0.007$ \\
\hline Small intestine & $0.304 \pm 0.025$ & $0.174 \pm 0.021$ & $0.016 \pm 0.001$ & $0.008 \pm 0.001$ & $0.006 \pm 0.001$ \\
\hline Large intestine & $0.857 \pm 0.061$ & $1.053 \pm 0.092$ & $0.052 \pm 0.012$ & $0.018 \pm 0.003$ & $0.013 \pm 0.003$ \\
\hline Kidney & $1.052 \pm 0.012$ & $0.884 \pm 0.020$ & $0.466 \pm 0.023$ & $0.343 \pm 0.045$ & $0.301 \pm 0.023$ \\
\hline Spleen & $0.057 \pm 0.007$ & $0.035 \pm 0.004$ & $0.028 \pm 0.002$ & $0.024 \pm 0.005$ & $0.021 \pm 0.001$ \\
\hline Pancreas & $0.016 \pm 0.002$ & $0.016 \pm 0.001$ & $0.008 \pm 0.002$ & $0.006 \pm 0.002$ & $0.005 \pm 0.001$ \\
\hline Stomach & $0.061 \pm 0.014$ & $0.053 \pm 0.014$ & $0.009 \pm 0.003$ & $0.005 \pm 0.001$ & $0.004 \pm 0.001$ \\
\hline Muscle & $0.017 \pm 0.004$ & $0.011 \pm 0.002$ & $0.006 \pm 0.003$ & $0.004 \pm 0.002$ & $0.003 \pm 0.002$ \\
\hline Fat & $0.014 \pm 0.004$ & $0.012 \pm 0.002$ & $0.009 \pm 0.001$ & $0.004 \pm 0.002$ & $0.004 \pm 0.001$ \\
\hline Bone & $0.092 \pm 0.009$ & $0.069 \pm 0.009$ & $0.080 \pm 0.008$ & $0.051 \pm 0.010$ & $0.046 \pm 0.006$ \\
\hline
\end{tabular}


Table S25. Biodistribution (\%ID/g) ${ }^{9-10}$ of $\left[{ }^{89} \mathrm{Zr}\right] \mathrm{Zr}-\mathrm{TRITA}$ in selected organs at $2,4,24,48$, and $72 \mathrm{~h}$ p.i.

\begin{tabular}{|c|c|c|c|c|c|}
\hline Tissue/Organ & $2 \mathrm{~h}$ & $4 h$ & $24 \mathrm{~h}$ & $48 h$ & $72 \mathrm{~h}$ \\
\hline Blood & $1.249 \pm 0.358$ & $1.142 \pm 0.349$ & $0.042 \pm 0.010$ & $0.010 \pm 0.003$ & $0.004 \pm 0.008$ \\
\hline Heart & $0.378 \pm 0.065$ & $0.314 \pm 0.084$ & $0.058 \pm 0.008$ & $0.066 \pm 0.012$ & $0.061 \pm 0.009$ \\
\hline Lung & $0.725 \pm 0.128$ & $0.583 \pm 0.187$ & $0.144 \pm 0.052$ & $0.141 \pm 0.043$ & $0.154 \pm 0.045$ \\
\hline Liver & $0.744 \pm 0.145$ & $0.543 \pm 0.118$ & $0.316 \pm 0.047$ & $0.380 \pm 0.045$ & $0.304 \pm 0.022$ \\
\hline Small intestine & $0.457 \pm 0.136$ & $0.345 \pm 0.062$ & $0.062 \pm 0.013$ & $0.054 \pm 0.007$ & $0.030 \pm 0.005$ \\
\hline Large intestine & $0.553 \pm 0.141$ & $0.804 \pm 0.176$ & $0.073 \pm 0.016$ & $0.051 \pm 0.007$ & $0.034 \pm 0.005$ \\
\hline Kidney & $1.271 \pm 0.218$ & $1.228 \pm 0.213$ & $0.681 \pm 0.015$ & $0.744 \pm 0.038$ & $0.588 \pm 0.036$ \\
\hline Spleen & $0.552 \pm 0.194$ & $0.287 \pm 0.084$ & $0.100 \pm 0.012$ & $0.161 \pm 0.030$ & $0.128 \pm 0.016$ \\
\hline Pancreas & $0.364 \pm 0.065$ & $0.274 \pm 0.037$ & $0.119 \pm 0.022$ & $0.136 \pm 0.026$ & $0.103 \pm 0.018$ \\
\hline Stomach & $0.198 \pm 0.038$ & $0.166 \pm 0.039$ & $0.040 \pm 0.014$ & $0.042 \pm 0.010$ & $0.020 \pm 0.004$ \\
\hline Muscle & $0.224 \pm 0.044$ & $0.173 \pm 0.026$ & $0.063 \pm 0.017$ & $0.061 \pm 0.035$ & $0.037 \pm 0.008$ \\
\hline Fat & $0.215 \pm 0.049$ & $0.153 \pm 0.023$ & $0.069 \pm 0.014$ & $0.091 \pm 0.014$ & $0.064 \pm 0.024$ \\
\hline Bone & $2.058 \pm 0.459$ & $3.329 \pm 0.220$ & $6.043 \pm 0.730$ & $10.805 \pm 0.803$ & $8.041 \pm 1.470$ \\
\hline
\end{tabular}


(a)

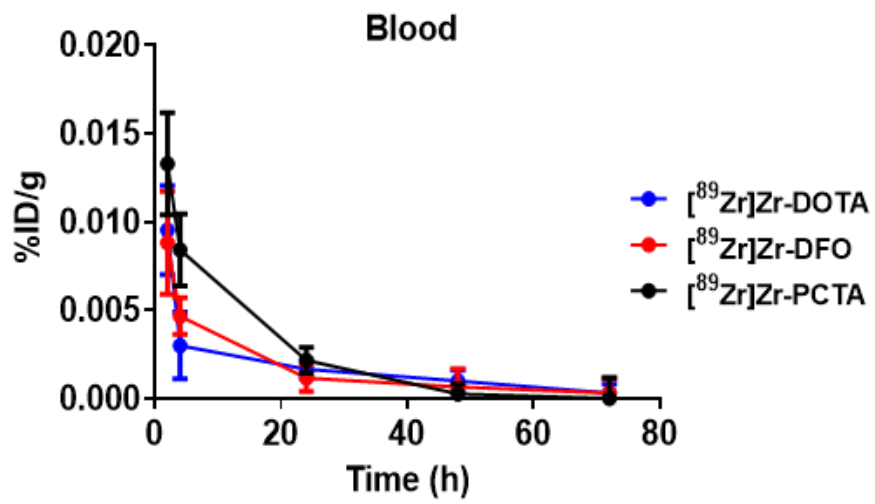

(c)

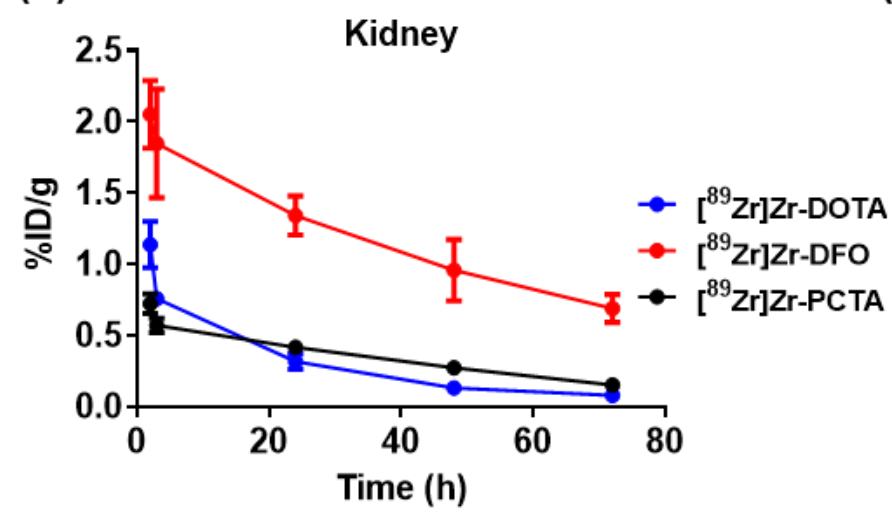

(b)

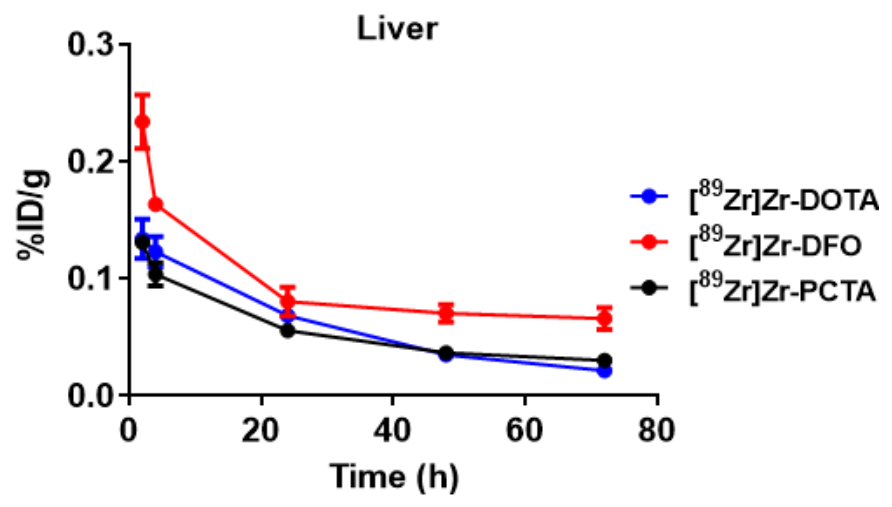

(d)

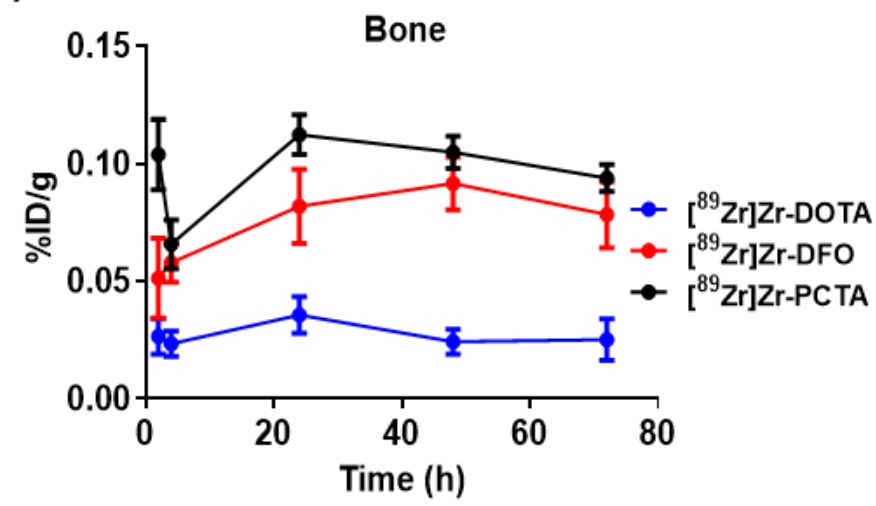

Figure S56. Biodistribution data summary of [ $\left.\left.{ }^{89} \mathrm{Zr}\right] \mathrm{Zr}-\mathrm{DOTA}{ }^{8},{ }^{89} \mathrm{Zr}\right] \mathrm{Zr}-\mathrm{DFO}{ }^{9}$, and $\left[{ }^{89} \mathrm{Zr}\right] \mathrm{Zr}$-PCTA in selected tissues: (a) blood, (b) liver, (c) kidney, and (d) bone. 
(a)

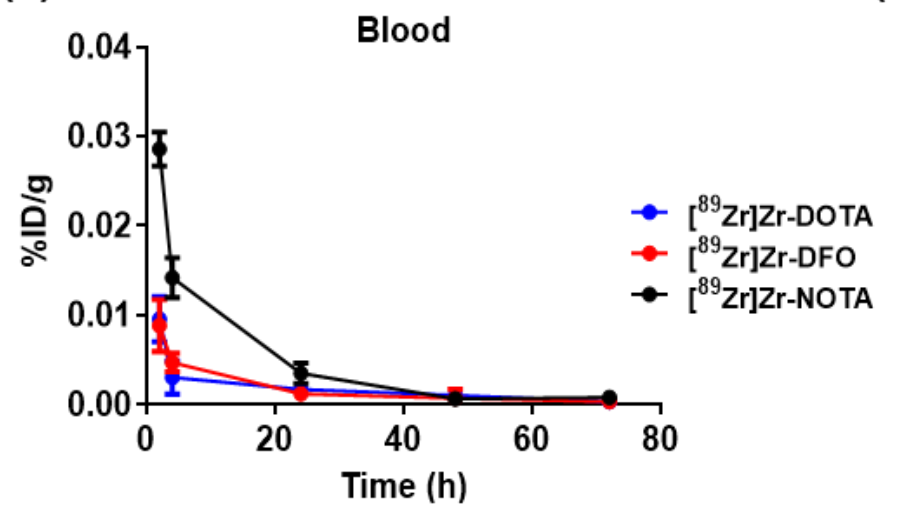

(c)

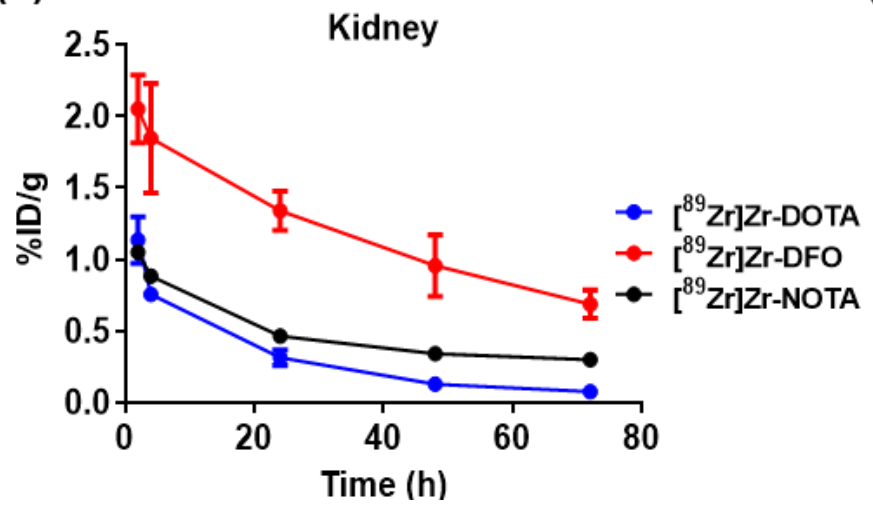

(b)

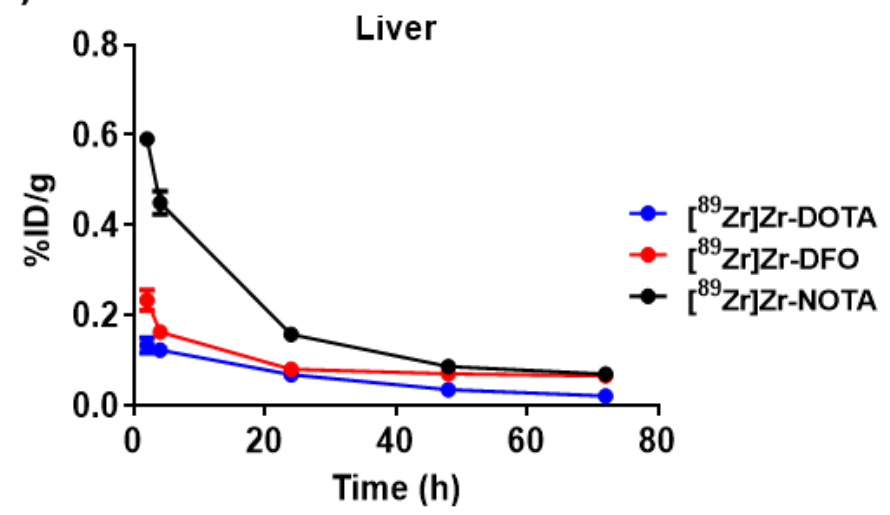

(d)

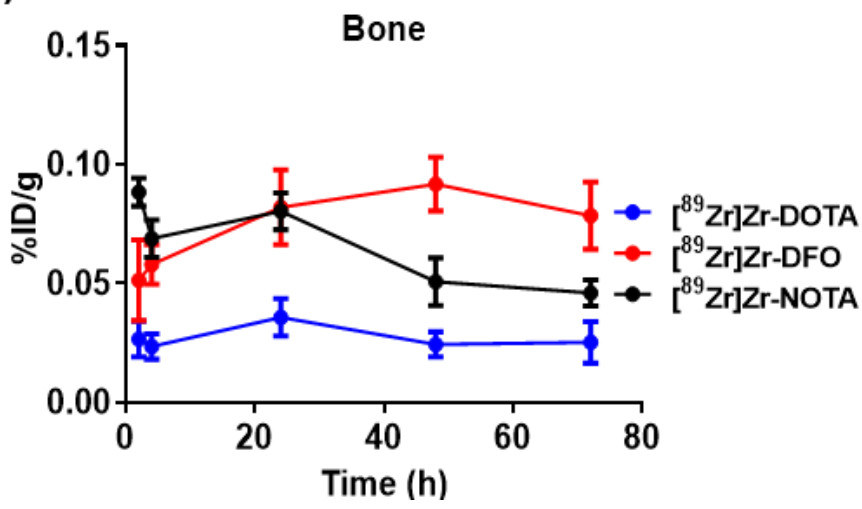

Figure S57. Biodistribution data summary of $\left.\left.{ }^{89} \mathrm{Zr}\right] \mathrm{Zr}-\mathrm{DOTA}{ }^{8},{ }^{89} \mathrm{Zr}\right] \mathrm{Zr}-\mathrm{DFO}{ }^{9}$, and $\left.{ }^{89} \mathrm{Zr}\right] \mathrm{Zr}$-NOTA in selected tissues: (a) blood, (b) liver, (c) kidney, and (d) bone. 
(a)

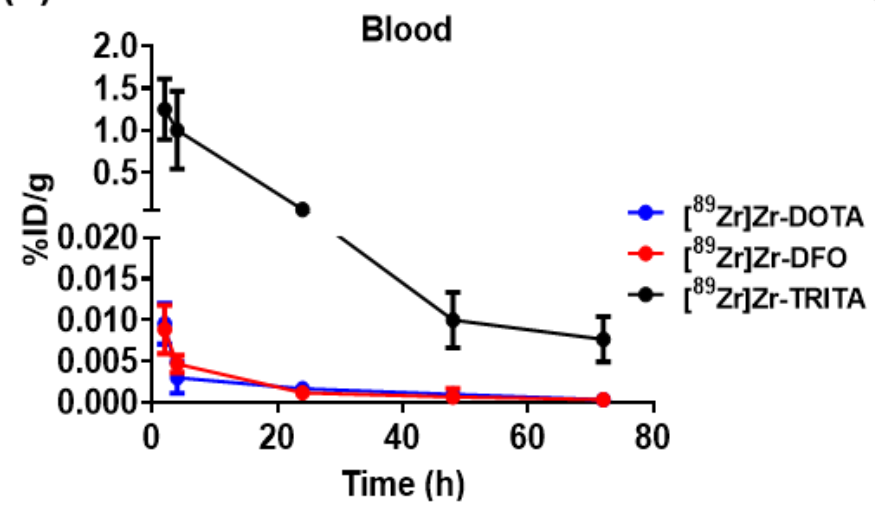

(c)

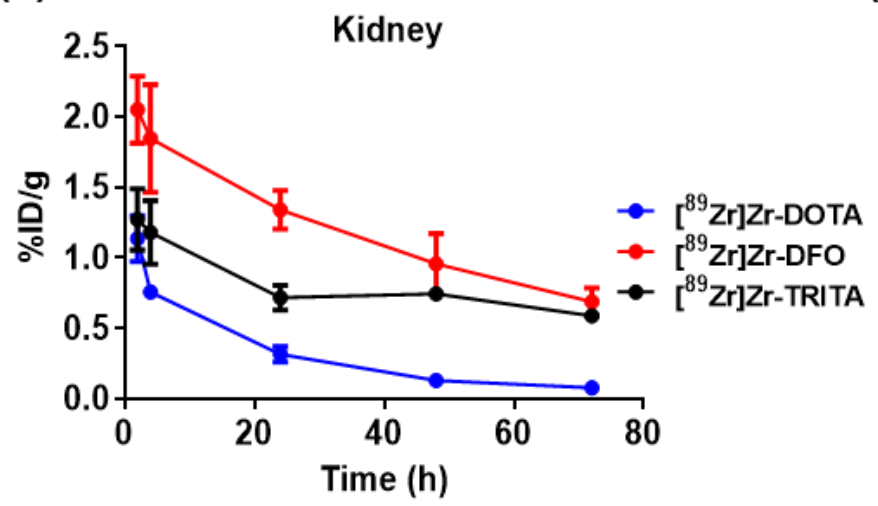

(b)

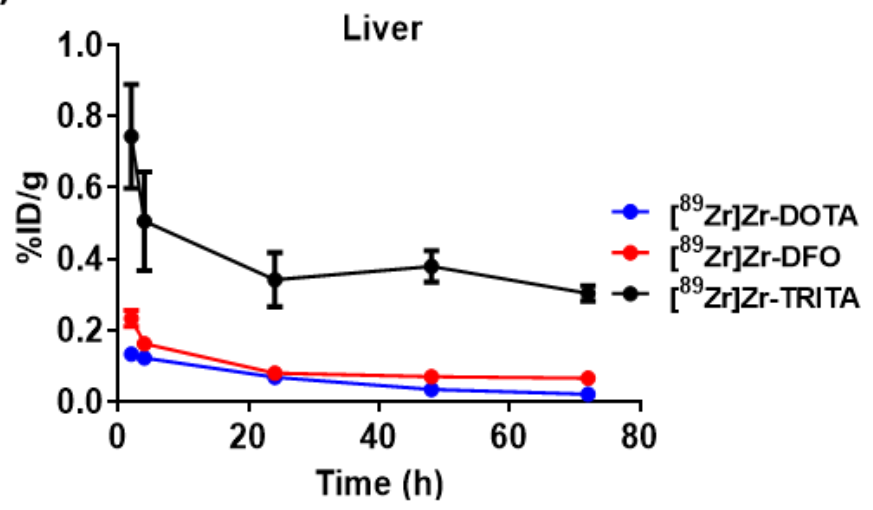

(d)

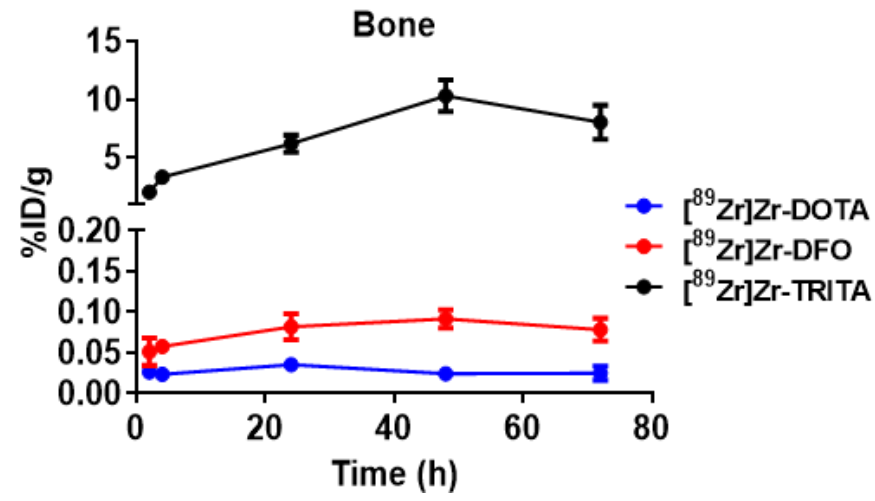

Figure S58. Biodistribution data summary of $\left[{ }^{89} \mathrm{Zr}\right] \mathrm{Zr}$-DOTA ${ }^{8},\left[{ }^{89} \mathrm{Zr}\right] \mathrm{Zr}$-DFO ${ }^{9}$, and $\left.{ }^{89} \mathrm{Zr}\right] \mathrm{Zr}$-TRITA in selected tissues: (a) blood, (b) liver, (c) kidney, and (d) bone. 

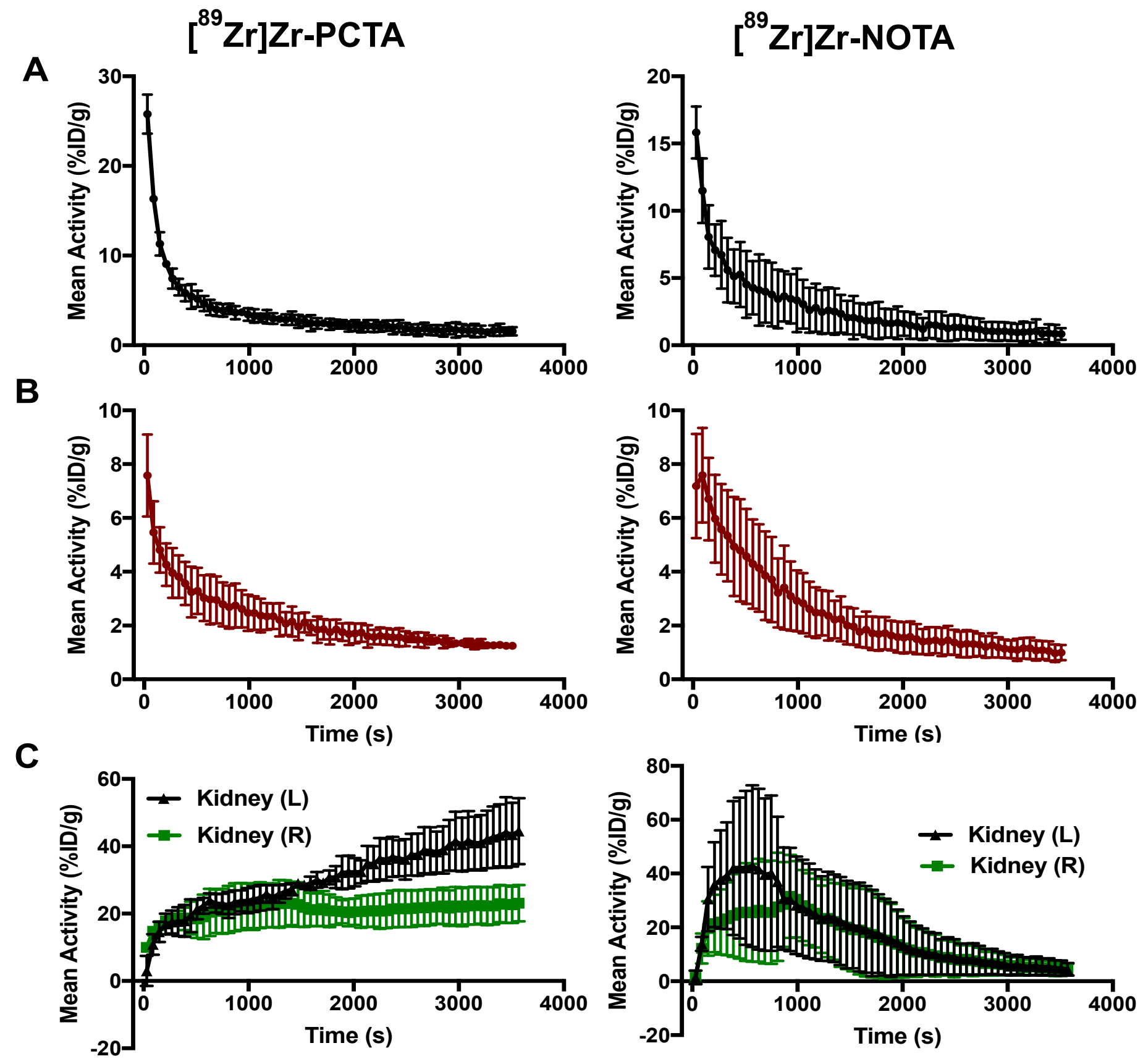

Figure S59. Dynamic PET data of $\left[{ }^{89} \mathrm{Zr}\right] \mathrm{Zr}$-PCTA and [ ${ }^{89} \mathrm{Zr}$ ]Zr-NOTA collected over the course of $1 \mathrm{~h}$ in specific tissues. Rapid perfusion and subsequent washout is represent via the blood (A) and liver (B). Slow accumulation is shown in the kidneys $(C)$ to enable rapid clearance, which is further depicted in static PET images at later time points. 
Table S26. Comparative stability of [ $\left.{ }^{89} \mathrm{Zr}\right] \mathrm{Zr}$-Complexes in exogenous ligand challenge study

\begin{tabular}{|c|c|c|c|c|}
\hline$\left[{ }^{89} \mathrm{Zr}\right] \mathrm{Zr}$-Complexes & Challenging Ligand & $\mathrm{pH}$ & Time Point & $\%$ Intact of ${ }^{89} \mathrm{Zr}$-complexes \\
\hline$\left[{ }^{89} \mathrm{Zr}\right] \mathrm{Zr}-\mathrm{L} 4{ }^{11}$ & EDTA (1000-fold) & 7.0 & $6 d$ & $87 \pm 1$ \\
\hline$\left[{ }^{89} \mathrm{Zr}\right] \mathrm{Zr}-\mathrm{TAM}-1^{9}$ & DTPA (1000-fold) & 7.0 & $7 d$ & 100 \\
\hline$\left[{ }^{89} \mathrm{Zr}\right] \mathrm{Zr}-\mathrm{TAM}-2^{9}$ & DTPA (1000-fold) & 7.0 & $7 d$ & 100 \\
\hline$\left[{ }^{89} \mathrm{Zr}\right] \mathrm{Zr}-2,3-\mathrm{HOPO}^{12}$ & DTPA (1000-fold) & 7.0 & $7 d$ & 78 \\
\hline$\left[{ }^{89} \mathrm{Zr}\right] \mathrm{Zr}-\mathrm{C} 7^{13}$ & EDTA (1750-fold) & 7.0 & $7 d$ & $87 \pm 3$ \\
\hline$\left[{ }^{89} \mathrm{Zr}\right] \mathrm{Zr}-\mathrm{CP} 256^{14}$ & \multicolumn{4}{|c|}{ Data Not Reported } \\
\hline \multirow{2}{*}[{}^{89}\mathrm{Zr}]{$\mathrm{Zr}-\mathrm{TAFC}{ }^{15}$} & \multirow{2}{*}{ EDTA (1000-fold) } & 7.0 & $7 d$ & $97.2 \pm 0.2$ \\
\hline & & 6.0 & $7 d$ & $94.4 \pm 0.5$ \\
\hline$\left[{ }^{89} \mathrm{Zr}\right] \mathrm{Zr}-\mathrm{DFO}{ }^{* 16}$ & \multicolumn{4}{|c|}{ Data Not Reported } \\
\hline$\left[{ }^{89} \mathrm{Zr}\right] \mathrm{Zr}$-DFOSq-Taur ${ }^{17}$ & EDTA (500-fold) & 7.0 & $1 d$ & $88 \pm 3.2$ \\
\hline$\left[{ }^{89} \mathrm{Zr}\right] \mathrm{Zr}$-(oxinate) $4^{18}$ & \multicolumn{4}{|c|}{ Data Not Reported } \\
\hline$\left[{ }^{89} \mathrm{Zr}\right] \mathrm{Zr}-(\mathrm{Me}-\mathrm{AHA}) 4^{19}$ & \multicolumn{4}{|c|}{ Data Not Reported } \\
\hline \multirow{2}{*}[{}^{89}\mathrm{Zr}]{$\mathrm{Zr}-\mathrm{THPN}{ }^{20}$} & \multirow{2}{*}{ EDTA (100-fold) } & 7.0 & $7 d$ & 100 \\
\hline & & 5.0 & $7 d$ & $41.6 \pm 11.1$ \\
\hline [89ZZ]Zr-DFO-HOPO ${ }^{21}$ & EDTA (100-fold) & 7.0 & $7 d$ & $>99$ \\
\hline \multirow{2}{*}[{}^{89}\mathrm{Zr}]{$\mathrm{Zr}-4 \mathrm{HMS}^{22}$} & \multirow{2}{*}{ DTPA (1000-fold) } & 7.0 & $7 d$ & $91.9 \pm 0.1$ \\
\hline & & 5.0 & $7 d$ & $80.8 \pm 1.8$ \\
\hline \multirow{2}{*}[{}^{89}\mathrm{Zr}]{$\mathrm{Zr}-(\mathrm{DFO} 2)^{23}$} & \multirow{2}{*}{ EDTA (100-fold) } & 7.0 & $7 d$ & $85.3 \pm 2.7$ \\
\hline & & 5.0 & $7 d$ & $0.6 \pm 0.2$ \\
\hline \multirow{2}{*}[{}^{89}\mathrm{Zr}]{$\mathrm{Zr}-\mathrm{HOPO}{ }^{24}$} & \multirow{2}{*}{ EDTA (100-fold) } & 7.0 & $7 d$ & 100 \\
\hline & & 5.0 & $7 d$ & $99.2 \pm 1.5$ \\
\hline \multirow{2}{*}[{}^{89}\mathrm{Zr}]{$\mathrm{Zr}-\mathrm{DFO}{ }^{8}$} & \multirow{2}{*}{ EDTA (1000-fold) } & 7.0 & $7 d$ & $20.3 \pm 0.5$ \\
\hline & & 5.0 & $7 d$ & 0 \\
\hline \multirow{2}{*}{$\left.{ }^{89} \mathrm{Zr}\right] \mathrm{Zr}-\mathrm{DOTA}{ }^{8}$} & \multirow{2}{*}{ EDTA (1000-fold) } & 7.0 & $7 d$ & 100 \\
\hline & & 5.0 & $7 d$ & 100 \\
\hline \multirow{2}{*}{$\begin{array}{l}{\left[{ }^{89} \mathrm{Zr}\right] \mathrm{Zr}-\mathrm{PCTA}} \\
\text { (Current Study) }\end{array}$} & \multirow{2}{*}{ EDTA (1000-fold) } & 7.0 & $7 d$ & 100 \\
\hline & & 5.0 & $7 d$ & 100 \\
\hline \multirow{2}{*}{$\begin{array}{l}{\left[{ }^{89} \mathrm{Zr}\right] \mathrm{Zr}-\mathrm{NOTA}} \\
\text { (Current Study) }\end{array}$} & \multirow{2}{*}{ EDTA (1000-fold) } & 7.0 & $7 d$ & $70.7 \pm 1.0$ \\
\hline & & 5.0 & $7 d$ & $65.3 \pm 1.2$ \\
\hline \multirow{2}{*}{$\begin{array}{l}{\left[{ }^{89} \mathrm{Zr}\right] \mathrm{Zr} \text {-TRITA }} \\
\text { (Current Study) }\end{array}$} & \multirow{2}{*}{ EDTA (1000-fold) } & 7.0 & $7 d$ & 0 \\
\hline & & 5.0 & $7 d$ & $9.6 \pm 0.1$ \\
\hline
\end{tabular}




\begin{tabular}{|c|c|c|c|c|c|c|c|c|}
\hline \multirow{2}{*}[{}^{89}\mathrm{Zr}]{$\mathrm{Zr}$-Complexes } & \multirow{2}{*}{$\begin{array}{l}\text { Time } \\
\text { Point }\end{array}$} & \multicolumn{7}{|c|}{$\%$ Intact of $\left[{ }^{89} \mathrm{Zr}\right] \mathrm{Zr}$-Complexes } \\
\hline & & $\mathrm{Fe}^{3+}$ & $\mathrm{Zn}^{2+}$ & $\mathrm{Co}^{2+}$ & $\mathrm{Cu}^{2+}$ & $\mathrm{Mg}^{2+}$ & $\mathrm{Gd}^{3+}$ & $\mathrm{Ga}^{3+}$ \\
\hline$\left[{ }^{89} \mathrm{Zr}\right] Z r-L 4{ }^{11}$ & & \multicolumn{7}{|c|}{ Data Not Reported } \\
\hline$\left.{ }^{[89} \mathrm{Zr}\right] \mathrm{Zr}-\mathrm{TAM}-1^{9}$ & & \multicolumn{7}{|c|}{ Data Not Reported } \\
\hline$\left.{ }^{[89} \mathrm{Zr}\right] \mathrm{Zr}-\mathrm{TAM}-2^{9}$ & & \multicolumn{7}{|c|}{ Data Not Reported } \\
\hline$\left[{ }^{89} \mathrm{Zr}\right] \mathrm{Zr}-2,3-\mathrm{HOPO}^{12}$ & & \multicolumn{7}{|c|}{ Data Not Reported } \\
\hline$\left[{ }^{89} \mathrm{Zr}\right] \mathrm{Zr}-\mathrm{C} 7^{13}$ & & \multicolumn{7}{|c|}{ Data Not Reported } \\
\hline$\left[{ }^{89} \mathrm{Zr}\right] \mathrm{Zr}-\mathrm{CP} 256^{14}$ & $\begin{array}{l}20 \\
\min \end{array}$ & \multicolumn{7}{|c|}{ Data Not Reported } \\
\hline$\left[{ }^{89} \mathrm{Zr}\right] Z r-T A F C^{15}$ & & \multicolumn{7}{|c|}{ Data Not Reported } \\
\hline$\left[{ }^{89} \mathrm{Zr}\right] \mathrm{Zr}-\mathrm{DFO}{ }^{* 16}$ & & \multicolumn{7}{|c|}{ Data Not Reported } \\
\hline$\left[{ }^{89} \mathrm{Zr}\right] Z r-D F O S q-T a u r 17$ & & \multicolumn{7}{|c|}{ Data Not Reported } \\
\hline$\left[{ }^{89} Z r\right] Z r-(o x i n a t e) 4^{18}$ & & \multicolumn{7}{|c|}{ Data Not Reported } \\
\hline$\left[{ }^{89} \mathrm{Zr}\right] \mathrm{Zr}-(\mathrm{Me}-\mathrm{AHA})_{4}{ }^{19}$ & & \multicolumn{7}{|c|}{ Data Not Reported } \\
\hline$\left[{ }^{89} \mathrm{Zr}\right] Z \mathrm{Zr}-\mathrm{THPN}{ }^{20}$ & & \multicolumn{7}{|c|}{ Data Not Reported } \\
\hline$\left[{ }^{89} \mathrm{Zr}\right] \mathrm{Zr}-\mathrm{DFO}-\mathrm{HOPO}{ }^{21}$ & & \multicolumn{7}{|c|}{ Data Not Reported } \\
\hline$\left[{ }^{89} \mathrm{Zr}\right] \mathrm{Zr}-4 \mathrm{HMS}^{22}$ & $7 d$ & $97.5 \pm 1.0$ & - & $99.0 \pm 0.7$ & $99.6 \pm 0.4$ & $99.6 \pm 0.4$ & - & - \\
\hline$\left[{ }^{89} \mathrm{Zr}\right] \mathrm{Zr}-(\mathrm{DFO} 2)^{23}$ & $7 d$ & $\sim 30$ & & \multicolumn{3}{|c|}{ Data Not Reported } & & \\
\hline$\left[{ }^{89} \mathrm{Zr}\right] \mathrm{Zr}-\mathrm{HOPO}{ }^{24}$ & $7 d$ & $83.0 \pm 4.2$ & $98.3 \pm 2.4$ & $98.9 \pm 1.6$ & $98.4 \pm 2.3$ & $98.7 \pm 1.8$ & $94.5 \pm 4.1$ & $96.4 \pm 0.6$ \\
\hline$\left[{ }^{89} \mathrm{Zr}\right] \mathrm{Zr}-\mathrm{DFO}{ }^{8}$ & $7 d$ & \multicolumn{2}{|c|}{$33.9 \pm 1.595 .9 \pm 0.7$} & $95.4 \pm 0.9$ & $96.0 \pm 1.0$ & $96.8 \pm 1.2 \varsigma$ & $96.8 \pm 0.2$ & $72.6 \pm 1.3$ \\
\hline$\left.{ }^{89} \mathrm{Zr}\right] \mathrm{Zr}-\mathrm{DOTA}{ }^{8}$ & $7 d$ & 100 & 100 & 100 & 100 & 100 & 100 & 100 \\
\hline $\begin{array}{l}{\left[{ }^{89} \mathrm{Zr}\right] \mathrm{Zr}-\mathrm{PCTA}} \\
\text { (Current Study) }\end{array}$ & $7 d$ & 100 & 100 & 100 & 100 & 100 & 100 & 100 \\
\hline $\begin{array}{l}\left.{ }^{89} \mathrm{Zr}\right] \mathrm{Zr}-\mathrm{NOTA} \\
\text { (Current Study) }\end{array}$ & $7 d$ & $96.2 \pm 0.2$ & $90.4 \pm 0.4$ & $92.9 \pm 0.7$ & $87.8 \pm 0.9$ & $90.6 \pm 0.4 \varsigma$ & $96.3 \pm 0.4$ & $89.5 \pm 1.5$ \\
\hline $\begin{array}{l}\left.{ }^{89} \mathrm{Zr}\right] \mathrm{Zr} \text {-TRITA } \\
\text { (Current Study) }\end{array}$ & $7 d$ & $70.4 \pm 0.8$ & $9.4 \pm 0.7$ & $7.3 \pm 0.6$ & $59.7 \pm 0.5$ & $83.9 \pm 0.5$ & $57.2 \pm 0.6$ & $61.8 \pm 1.2$ \\
\hline
\end{tabular}


Table S28. Comparative biodistribution results of $\left[{ }^{89} \mathrm{Zr}\right] \mathrm{Zr}$-Complexes

\begin{tabular}{|c|c|c|c|c|c|}
\hline \multirow{2}{*}[{}^{89}\mathrm{Zr}]{$\mathrm{Zr}-\mathrm{Complexes}$} & \multirow{2}{*}{$\begin{array}{l}\text { Time } \\
\text { Point }\end{array}$} & \multicolumn{4}{|c|}{$\%$ ID $/ g$} \\
\hline & & Blood & Liver & Kidney & Bone \\
\hline$\left[{ }^{89} \mathrm{Zr}\right] \mathrm{Zr}-\mathrm{L} 4{ }^{11}$ & $24 \mathrm{~h}$ & $0.09 \pm 0.01$ & $0.40 \pm 0.14$ & $2.76 \pm 0.40$ & $0.60 \pm 0.19$ \\
\hline$\left[{ }^{89} \mathrm{Zr}\right] \mathrm{Zr}-\mathrm{TAM}-1^{9}$ & $24 \mathrm{~h}$ & $0.003 \pm 0.002$ & $0.449 \pm 0.037$ & $8.214 \pm 1.018$ & $0.100 \pm 0.030$ \\
\hline$\left[{ }^{89} \mathrm{Zr}\right] \mathrm{Zr}-\mathrm{TAM}-2^{9}$ & $24 \mathrm{~h}$ & $0.010 \pm 0.003$ & $1.244 \pm 0.180$ & $46.095 \pm 7.788$ & $0.274 \pm 0.100$ \\
\hline$\left[{ }^{89} \mathrm{Zr}\right] \mathrm{Zr}-2,3-\mathrm{HOPO}^{12}$ & $24 \mathrm{~h}$ & $0.004 \pm 0.001$ & $0.650 \pm 0.080$ & $29.191 \pm 6.989$ & $0.272 \pm 0.066$ \\
\hline$\left[{ }^{89} \mathrm{Zr}\right] \mathrm{Zr}-\mathrm{C} 7^{13}$ & & \multicolumn{4}{|c|}{ Data Not Reported } \\
\hline$\left[{ }^{89} \mathrm{Zr}\right] \mathrm{Zr}-\mathrm{CP} 256^{14}$ & & \multicolumn{4}{|c|}{ Data Not Reported } \\
\hline$\left[{ }^{89} \mathrm{Zr}\right] \mathrm{Zr}-\mathrm{TAFC}{ }^{15}$ & $6 \mathrm{~h}$ & $0.05 \pm 0.01$ & - & $0.86 \pm 0.48$ & $0.04 \pm 0.02$ \\
\hline$\left[{ }^{89} \mathrm{Zr}\right] \mathrm{Zr}-\mathrm{DFO}{ }^{* 16}$ & & \multicolumn{4}{|c|}{ Data Not Reported } \\
\hline$\left.{ }^{89} \mathrm{Zr}\right] \mathrm{Zr}-\mathrm{DFOSq}-\mathrm{Taur}^{17}$ & & \multicolumn{4}{|c|}{ Data Not Reported } \\
\hline$\left[{ }^{89} \mathrm{Zr}\right] \mathrm{Zr}$-(oxinate) $4^{18}$ & & \multicolumn{4}{|c|}{ Data Not Reported } \\
\hline$\left[{ }^{89} \mathrm{Zr}\right] \mathrm{Zr}-(\mathrm{Me}-\mathrm{AHA}) 4^{19}$ & & \multicolumn{4}{|c|}{ Data Not Reported } \\
\hline$\left[{ }^{89} \mathrm{Zr}\right] \mathrm{Zr}-\mathrm{THPN}{ }^{20}$ & $24 \mathrm{~h}$ & $0.01 \pm 0.01$ & $1.08 \pm 1.25$ & $3.49 \pm 0.28$ & $011 \pm 0.01$ \\
\hline$\left[{ }^{89} \mathrm{Zr}\right] \mathrm{Zr}-\mathrm{DFO}-\mathrm{HOPO}{ }^{21}$ & $24 \mathrm{~h}$ & $0.00 \pm 0.00$ & $0.02 \pm 0.00$ & $0.08 \pm 0.01$ & $0.01 \pm 0.00$ \\
\hline$\left[{ }^{89} \mathrm{Zr}\right] \mathrm{Zr}-4 \mathrm{HMS}^{22}$ & $24 \mathrm{~h}$ & $0.00 \pm 0.00$ & $0.04 \pm 0.00$ & $0.80 \pm 0.20$ & $0.01 \pm 0.00$ \\
\hline$\left[{ }^{89} \mathrm{Zr}\right] \mathrm{Zr}-(\mathrm{DFO} 2)^{23}$ & & \multicolumn{4}{|c|}{ Data Not Reported } \\
\hline$\left[{ }^{89} \mathrm{Zr}\right] \mathrm{Zr}-\mathrm{HOPO}{ }^{24}$ & $24 \mathrm{~h}$ & $0.02 \pm 0.00$ & $0.06 \pm 0.03$ & $0.51 \pm 0.29$ & $0.17 \pm 0.03$ \\
\hline$\left[{ }^{89} \mathrm{Zr}\right] \mathrm{Zr}-\mathrm{DFO}{ }^{8}$ & $24 \mathrm{~h}$ & $0.00 \pm 0.00$ & $0.08 \pm 0.01$ & $1.34 \pm 0.14$ & $0.08 \pm 0.02$ \\
\hline$\left[{ }^{89} \mathrm{Zr}\right] \mathrm{Zr}-\mathrm{DOT} \mathrm{A}^{8}$ & $24 \mathrm{~h}$ & $0.00 \pm 0.00$ & $0.07 \pm 0.00$ & $0.32 \pm 0.05$ & $0.04 \pm 0.01$ \\
\hline $\begin{array}{l}{\left[{ }^{89} \mathrm{Zr}\right] \mathrm{Zr}-\mathrm{PCTA}} \\
\text { (Current Study) }\end{array}$ & $24 \mathrm{~h}$ & $0.00 \pm 0.00$ & $0.06 \pm 0.00$ & $0.42 \pm 0.02$ & $0.11 \pm 0.01$ \\
\hline $\begin{array}{l}\left.{ }^{89} \mathrm{Zr}\right] \mathrm{Zr}-\mathrm{NOTA} \\
\text { (Current Study) }\end{array}$ & $24 \mathrm{~h}$ & $0.00 \pm 0.00$ & $0.16 \pm 0.01$ & $0.47 \pm 0.02$ & $0.08 \pm 0.01$ \\
\hline $\begin{array}{l}\left.{ }^{89} \mathrm{Zr}\right] \mathrm{Zr}-\mathrm{TRITA} \\
\text { (Current Study) }\end{array}$ & $24 \mathrm{~h}$ & $0.04 \pm 0.01$ & $0.32 \pm 0.05$ & $0.68 \pm 0.02$ & $6.04 \pm 0.73$ \\
\hline
\end{tabular}




\section{REFERENCES}

1. Bruker APEX2 2014.11-0; Bruker AXS Inc.: Madison, Wisconsin, USA, 2014.

2. Bruker APEX3 2016.1-0; Bruker AXS Inc.: Madison, Wisconsin, USA., 2016.

3. Sheldrick, G. M., A short history of SHELX. Acta Crystallogr., Sect. A: Found. Crystallogr. 2008, 64 (1), 112-122.

4. SAINT, 8.34A; Bruker AXS Inc.: Madison, Wisconsin, USA, 2014.

5. Sheldrick, G. M. SHELXL, 2014/7; Bruker AXS Inc.: Madison, Wisconsin, USA., 2014.

6. Sheldrick, G. M. SADABS, 2014/5; University of Göttingen, Germany, 2014.

7. Waterhouse, R. N., Determination of lipophilicity and its use as a predictor of blood-brain barrier penetration of molecular imaging agents. Mol Imaging Biol 2003, 5 (6), 376-389.

8. Pandya, D. N.; Bhatt, N.; Yuan, H.; Day, C. S.; Ehrmann, B. M.; Wright, M.; Bierbach, U.; Wadas, T. J., Zirconium tetraazamacrocycle complexes display extraordinary stability and provide a new strategy for zirconium-89-based radiopharmaceutical development. Chem. Sci. 2017, 8 (3), 2309-2314.

9. Pandya, D. N.; Pailloux, S.; Tatum, D.; Magda, D.; Wadas, T. J., Di-macrocyclic terephthalamide ligands as chelators for the PET radionuclide zirconium-89. Chem. Commun. (Cambridge, U. K.) 2015, 51 (12), 2301-2303.

10. Sprague, J. E.; Peng, Y.; Fiamengo, A. L.; Woodin, K. S.; Southwick, E. A.; Weisman, G. R.; Wong, E. H.; Golen, J. A.; Rheingold, A. L.; Anderson, C. J., Synthesis, characterization and in vivo studies of $\mathrm{Cu}$ (II)-64labeled cross-bridged tetraazamacrocycle-amide complexes as models of peptide conjugate imaging agents. J Med Chem 2007, 50 (10), 2527-2535.

11. Boros, E.; Holland, J. P.; Kenton, N.; Rotile, N.; Caravan, P., Macrocycle-Based Hydroxamate Ligands for Complexation and Immunoconjugation of (89)Zirconium for Positron Emission Tomography (PET) Imaging. Chempluschem 2016, 81 (3), 274-281.

12. Tinianow, J. N.; Pandya, D. N.; Pailloux, S. L.; Ogasawara, A.; Vanderbilt, A. N.; Gill, H. S.; Williams, S.P.; Wadas, T. J.; Magda, D.; Marik, J., Evaluation of a 3-hydroxypyridin-2-one (2,3-HOPO) based macrocyclic chelator for $89 \mathrm{Zr} 4+$ and its use for immunoPET imaging of HER2 positive model of ovarian carcinoma in mice. Theranostics 2016, 6 (4), 511-521.

13. Guerard, F.; Lee, Y.-S.; Brechbiel, M. W., Rational Design, Synthesis, and Evaluation of Tetrahydroxamic Acid Chelators for Stable Complexation of Zirconium(IV). Chem. - Eur. J. 2014, 20 (19), 5584-5591.

14. Ma, M. T.; Meszaros, L. K.; Paterson, B. M.; Berry, D. J.; Cooper, M. S.; Ma, Y.; Hider, R. C.; Blower, P. J., Tripodal tris(hydroxypyridinone) ligands for immunoconjugate PET imaging with (89)Zr(4+): comparison with desferrioxamine-B. Dalton Trans 2015, 44 (11), 4884-4900.

15. Zhai, C.; Summer, D.; Rangger, C.; Franssen, G. M.; Laverman, P.; Haas, H.; Petrik, M.; Haubner, R.; Decristoforo, C., Novel Bifunctional Cyclic Chelator for (89)Zr Labeling-Radiolabeling and Targeting Properties of RGD Conjugates. Mol Pharm 2015, 12 (6), 2142-2150. 
16. Patra, M.; Bauman, A.; Mari, C.; Fischer, C. A.; Blacque, O.; Haussinger, D.; Gasser, G.; Mindt, T. L., An octadentate bifunctional chelating agent for the development of stable zirconium-89 based molecular imaging probes. Chem Commun 2014, 50 (78), 11523-11525.

17. Rudd, S. E.; Roselt, P.; Cullinane, C.; Hicks, R. J.; Donnelly, P. S., A desferrioxamine B squaramide ester for the incorporation of zirconium-89 into antibodies. Chem Commun (Camb) 2016, 52 (80), 11889-11892.

18. Ferris, T. J.; Charoenphun, P.; Meszaros, L. K.; Mullen, G. E. D.; Blower, P. J.; Went, M. J., Synthesis and characterisation of zirconium complexes for cell tracking with Zr-89 by positron emission tomography. Dalton Trans 2014, 43 (39), 14851-14857.

19. Guerard, F.; Lee, Y. S.; Tripier, R.; Szajek, L. P.; Deschamps, J. R.; Brechbiel, M. W., Investigation of $\mathrm{Zr}(\mathrm{IV})$ and $89 \mathrm{Zr}(\mathrm{IV})$ complexation with hydroxamates: progress towards designing a better chelator than desferrioxamine B for immuno-PET imaging. Chem Commun (Camb) 2013, 49 (10), 1002-1004.

20. Buchwalder, C.; Rodriguez-Rodriguez, C.; Schaffer, P.; Karagiozov, S. K.; Saatchi, K.; Hafeli, U. O., A new tetrapodal 3-hydroxy-4-pyridinone ligand for complexation of (89)zirconium for positron emission tomography (PET) imaging. Dalton Trans 2017, 46 (29), 9654-9663.

21. Allott, L.; Da Pieve, C.; Meyers, J.; Spinks, T.; Ciobota, D. M.; Kramer-Marek, G.; Smith, G., Evaluation of DFO-HOPO as an octadentate chelator for zirconium-89. Chem Commun (Camb) 2017, 53 (61), 85298532.

22. Alnahwi, A. H.; Ait-Mohand, S.; Dumulon-Perreault, V.; Dory, Y. L.; Guerin, B., Promising Performance of 4HMS, a New Zirconium-89 Octadendate Chelator. ACS Omega 2020, 5 (19), 10731-10739.

23. Sarbisheh, E. K.; Salih, A. K.; Raheem, S. J.; Lewis, J. S.; Price, E. W., A High-Denticity Chelator Based on Desferrioxamine for Enhanced Coordination of Zirconium-89. Inorg. Chem. 2020, 59 (16), 11715-11727.

24. Deri, M. A.; Ponnala, S.; Zeglis, B. M.; Pohl, G.; Dannenberg, J. J.; Lewis, J. S.; Francesconi, L. C., Alternative chelator for ${ }^{89} \mathrm{Zr}$ radiopharmaceuticals: radiolabeling and evaluation of 3,4,3-(LI-1,2-HOPO). $J$ Med Chem 2014, 57 (11), 4849-4860. 